\title{
Costs and effects of Doppler ultrasound measurements in suspected intrauterine growth retardation : a randomised clinical trial
}

Citation for published version (APA):

Nienhuis, S. J. (1995). Costs and effects of Doppler ultrasound measurements in suspected intrauterine growth retardation : a randomised clinical trial. [Doctoral Thesis, Maastricht University]. Datawyse / Universitaire Pers Maastricht. https://doi.org/10.26481/dis.19950216sn

Document status and date:

Published: 01/01/1995

DOI:

10.26481/dis.19950216sn

Document Version:

Publisher's PDF, also known as Version of record

Please check the document version of this publication:

- A submitted manuscript is the version of the article upon submission and before peer-review. There can be important differences between the submitted version and the official published version of record.

People interested in the research are advised to contact the author for the final version of the publication, or visit the DOI to the publisher's website.

- The final author version and the galley proof are versions of the publication after peer review.

- The final published version features the final layout of the paper including the volume, issue and page numbers.

Link to publication

\footnotetext{
General rights rights.

- You may freely distribute the URL identifying the publication in the public portal. please follow below link for the End User Agreement:

www.umlib.nl/taverne-license

Take down policy

If you believe that this document breaches copyright please contact us at:

repository@maastrichtuniversity.nl

providing details and we will investigate your claim.
}

Copyright and moral rights for the publications made accessible in the public portal are retained by the authors and/or other copyright owners and it is a condition of accessing publications that users recognise and abide by the legal requirements associated with these

- Users may download and print one copy of any publication from the public portal for the purpose of private study or research.

- You may not further distribute the material or use it for any profit-making activity or commercial gain

If the publication is distributed under the terms of Article $25 \mathrm{fa}$ of the Dutch Copyright Act, indicated by the "Taverne" license above, 
Costs and effects of Doppler ultrasound measurements in suspected intrauterine growth retardation 


\section{CIP-DATA KONINKLIJKE BIBLIOTHEEK, DEN HAAG}

Nienhuis, Saskia Johanna

Costs and effects of Doppler ultrasound measurements in suspected intrauterine growth retardation: A randomised clinical trial / Saskia Johanna Nienhuis. - Maastricht :

Universitaire Pers Maastricht - Ill.

Thesis Maastricht. - With ref. - With summary in Dutch. ISBN 90-5278-174-5

Subject headings: Doppler ultrasonography ; cost-benefit analysis / growth retardation.

Book production: Datawyse | Universitaire Pers Maastricht

Cover design: A.L..1.. Casceleyn

Illustrations: R.J. Nienhuis 


\section{Costs and effects of Doppler ultrasound measurements in suspected intrauterine growth retardation \\ A randomised clinical trial}

ter verkrijging van de graad van doctor

aan de Rijksuniversiteit Limburg te Maastricht,

op gezag van de Rector Magnificus, prof. dr H. Philipsen

volgens het besluit van het College van Decanen,

in het openbaar te verdedigen

op donderdag 16 februari 1995 om 16.00 uur

door

Saskia Johanna Nienhuis 


\section{Promotor:}

Prof. dr J. de Haan

Co-promotor:

Dr H.J. Hoogland

Beoordelingscommissie:

Prof. dr C.E. Blanco (voorzitter)

Prof. dr J.A. Knottnerus

Prof. dr K. Maršál (University of Lund, Sweden)

Prof. dr R.S. Reneman

Prof. dr J.W. Wladimiroff (Erasmus Universiteit Rotterdam) 


\section{Paranimfen}

Ir K.J. Blok van Laer

Dr. C.S. Ruissen

Amber, Amelia, Angelo, Ashley, Ashwin, Asli, Bart, Bas, Benjamin, Birgit, Bram, Brian, Carmen, Caspar, Chantal, Chantal, Charelle, Charmaine, Christianne, Ciox, Claudia, Danielle, Danka, Daphne, Denise, Denise, Dennis, Desiree, Dominique, Dylan, Dyonne, Elizabeth, Esmee, Esmina, Eveline, Felica, Frederieke, George, Georgina, Gerald, Gita, Glendy, Guy, Gwenny, Hassan, Jaimie, Jaimie, Jamie, Jason, Jill, Jiri, Job, Jody, Joey, Jordy, Jordy, Joris, Joy, Kaotar, Kelly, Kelly, Kelsey, Kenny, Kevin, Leroy, Leroy, Leyla, Lieve, Maarten, Maik, Maikel, Maikel, Manon, Margaret, Marjolein, Mark, Mark, Marlijn, Martijn, Marvin, Maurice, Melvin, Michael, Michelle, Michelle, Michelle, Mick, Mitch, Mitch, Natasja, Nicky, Nicky, Nicole, Niels, Octave, Pascal, Patricia, Patrick, Peggy, Phillipe, Rachelle, Rafaela, Ralf, Ramona, Raoul, Raymond, Remi, Renate, Renee, Rianne, Ricardo, Rickey, Ricky, Roger, Rosita, Rowena, Rowena, Samantha, Samantha, Sandy, Sean, Serdan, Shirley, Shirley, Sinead, Sophie, Stefan, Stefan, Stefan, Stefan, Stefanie, Stephan, Stephanie, Stephanie, Stephanie, Steven, Sven, Sven, Tamara, Tiffany, Tom, Tom, Tuba, Verena, Viony, Xavier, Yanal, Youri, Yvonne, Yvonne, en hun ouders en verzorgers. 


\section{Contents}

\section{Chapter 1 Introduction 9}

Chapter 2 Review of the literature: Growth, intrauterine growth retardation, and its consequences 13

2.1 Normal growth 13

2.2 Defining intrauterine growth retardation 17

2.3 Aetiology of intrauterine growth retardation 18

2.4 Fetal growth in intrauterine growth retardation 19

2.5 Identification of the small fetus 20

Fundal height measurements 22

Ultrasonic fetal biometry 22

Doppler ultrasound measurements in the umbilical artery 27

2.6 Effectiveness of Doppler ultrasound measurements:

Randomised Clinical Trials 28

2.7 Perinatal consequences of intrauterine growth retardation 41 Postnatal (catch-up) growth 41

Mortality and morbidity 42

2.8 Therapeutic possibilities 44

2.9 Study objective 44

Chapter 3 Randomised Clinical Trial: Study Protocol 47

3.1 Study population 47

Research institute 47

Inclusion 48

Stratification and randomisation 49

3.2 Doppler ultrasound measurements in the umbilical artery 49

Technical background 50

Pathophysiology 52

Reproducibility 52

Safety 53

Measurement technique 53

Doppler ultrasound measurements in intervention and control group 54 
3.3 Follow-up 57

Costs 57

Perinatal outcome 61

Neurological development 61

Postnatal growth 62

3.4 Study size 67

3.5 Blinding 68

3.6 Data handling and statistical analysis 68

Chapter 4 Randomised Clinical Trial: Results 69

4.1 Results 69

Study population 69

Doppler ultrasound measurements $\quad 72$

Perinatal outcome 73

Costs 76

Neurological development $\quad 81$

Postnatal growth 83

4.2 Analysis 85

Costs 87

Perinatal outcome 89

Neurological development 91

Postnatal (catch-up) growth 91

4.3 Summary of the results 92

Chapter 5 Secondary analysis: Doppler ultrasound results versus outcome of the randomised clinical trial 93

5.1 Doppler ultrasound results versus costs 93

5.2 Doppler ultrasound results versus perinatal outcome 93

5.3 Doppler ultrasound results versus neurological development 96

5.4 Doppler ultrasound results versus postnatal growth 99

5.5 Summary of the results 102

Chapter 6 Randomised Clinical Trial: Discussion and Conclusions 103

6.1 Costs 104

6.2 Perinatal outcome 107

6.3 Neurological development 108

6.4 Postnatal growth 108

6.5 Conclusions 109 
Chapter 7 Questionnaire: Routine clinical policy and application of Doppler ultrasound measurements in suspected intrauterine growth retardation in university hospitals in the Netherlands 111

7.1 Introduction 111

7.2 Methods 113

7.3 Results 113

Response 113

Definitions and diagnosis of intrauterine growth retardation 113

Clinical policy in suspected intrauterine growth retardation 114 Estimation of the magnitude of the problem 115

Doppler ultrasound measurements in the umbilical artery 117

7.4 Discussion 117

Intrauterine growth retardation 117

Doppler ultrasound measurements in the umbilical artery 119

7.5 Conclusion 120

References 121

Abbreviations 135

Summary 137

Samenvatting 143

Appendix 1 Neurological examination of neonates at term according to Prechtl (Prechtl 1977) 149

Appendix 2 Neurological examination according to Touwen at the corrected age of 6 and 9 months (Touwen 1984) 150

Appendix 3 Anthropometric reference curves 151

Acknowledgements 153

Curriculum vitae $\quad 155$

Publications 156 


\section{Chapter 1}

\section{Introduction}

Intrauterine growth retardation (IUGR) is the condition whereby the fetus cannot fulfil its genetic growth potential. IUGR is associated with an increase in perinatal morbidity and mortality (Starfield 1982). Therefore, identification of fetal growth disturbances is a major objective in routine prenatal care. If IUGR is suspected, the risk of an insufficient intrauterine environment must be balanced against the risks of (preterm) extrauterine life in order to establish the optimal timing of delivery (Swyer 1992). However, the risks involved are difficult to quantify, and criteria for the optimal timing of delivery are equivocal. Studies evaluating diagnostic tests for IUGR and monitoring techniques for fetal wellbeing, therefore, duly receive a great deal of attention in the literature (Villar 1986).

The definition of IUGR itself has been the subject of several publications (Wilcox 1983, Altman 1989). In the literature, IUGR is defined by certain thresholds of birth weight ( $<2500$ grams), or of birth weight centiles $(<$ P2.3, < P10) for the gestational age. Moreover, various threshold values are used simultaneously in the different publications to distinguish normal from abnormal. Such thresholds, however, define those fetuses or neonates who are small for gestational age (SGA). That SGA is by no means synonymous with IUGR is illustrated in figure 1.1, where the absence or presence of SGA is plotted against the absence or presence of IUGR. The neonates on the left side of each square symbolise the genetic growth potential. The neonates on the right side symbolise the actual size reached. If there are no detrimental influences in the intrauterine environment, the fetus will grow according to its genetic profile. This is illustrated by showing two equally large neonates (IUGR $=\mathrm{no}$ ). When intrauterine conditions are not optimal, the fetus cannot fulfil its genetic growth potential and will turn out to be thinner and smaller than its genetic counterpart (IUGR = yes).

It is clear from figure 1.1, that growth retardation will not be restricted to fetuses in the lowest centile range. Alternatively, every centile threshold chosen to define IUGR will include normal, genetically small fetuses or neonates. This also occurs with the use of thresholds in ultrasonic fetal biometry or fundal height measurements. In conclusion, such definitions do not clarify the presence, severity, or the quality of growth retardation. As there is no definitive gold standard for IUGR, the above standards are often used in research and in clinical practice. However, in the absence of an absolute definition for IUGR, the validity of a diagnostic test for IUGR cannot be quantified. If a significant proportion of a SGA study population consists of genetically determined small fetuses, 


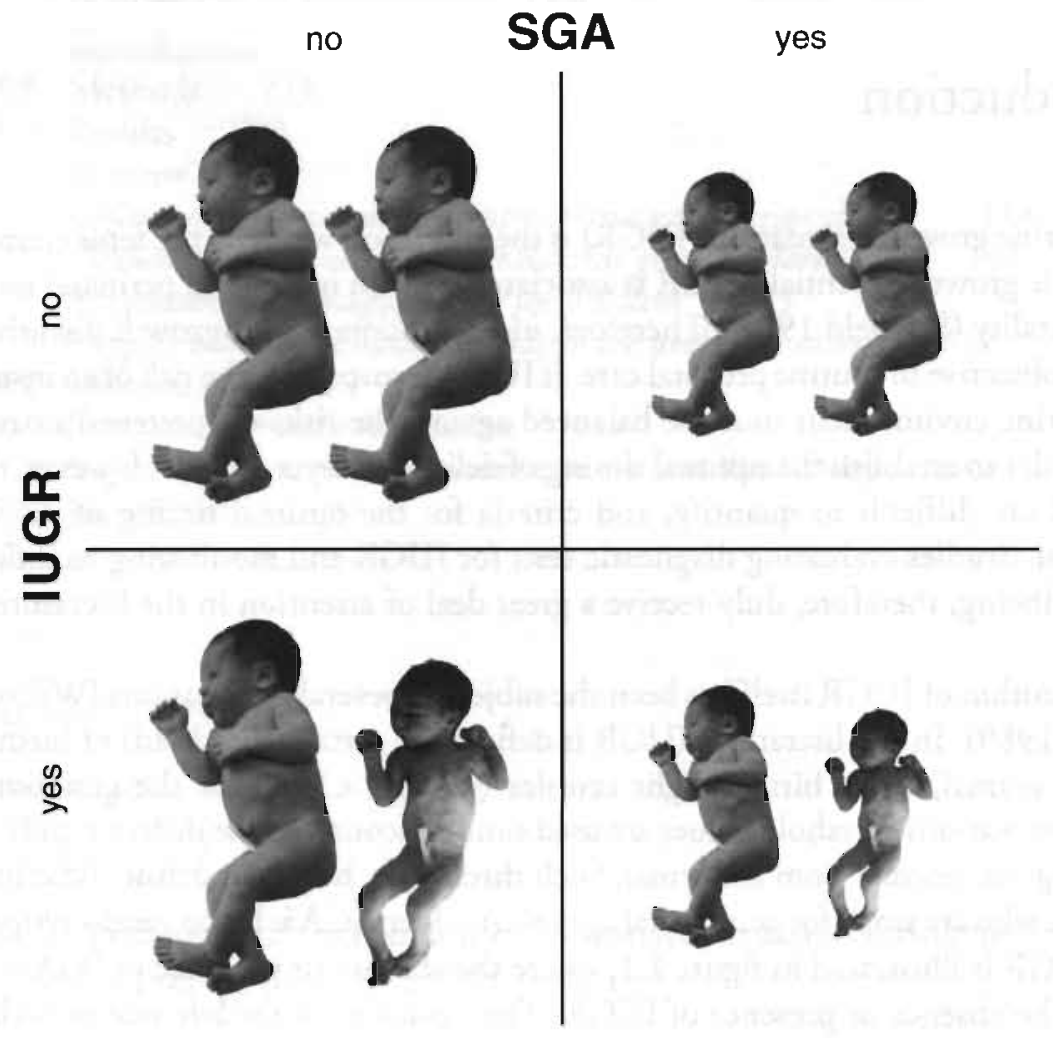

Figure 1.1

Small for gestational age versus intrauterine growth retardation

test parameters used to detect true growth retardation will falsely show low specificities, while the specificities of parameters related to smallness alone, will be unjustly high. If IUGR is suspected, genetic smallness and IUGR might be differentiated if uteroplacental function is assessed, in addition to fetal size. A compromised utero-placental circulation is thought to play an important role in the development of IUGR. Therefore, Doppler ultrasound assessment of the sufficiency of the fetal, placental, and maternal circulation has rapidly gained popularity in obstetrics (Nienhuis 1994). Most of the studies on the validity of blood flow velocity measurements in the umbilical artery concern the detection of the fetus at risk of IUGR. 
Some authors have suggested that suspected IUGR is a largely benign condition if it is associated with normal blood velocity profiles in the umbilical artery (Reuwer 1987, Haddad 1988, Burke 1990, Tyrrell 1990). This finding introduces a new angle to the problem of IUGR. Theoretically, a genetically-small/not-IUGR fetus is expected to have normal blood flow velocities, while the genuinely growth retarded fetus due to uteroplacental dysfunction, would show deviant Doppler parameters. Because raised Doppler ultrasound values have been found to be an early sign of a deteriorating intrauterine environment (Ribbert 1993), findings within the normal range indicate low fetal risk. In cases of suspected fetal growth retardation, normal Doppler values could be used to reassure both patient and doctor. In these low risk cases, unnecessary, costly clinical management can be prevented. Distinguishing IUGR from genetic smallness is, therefore, attractive from a cost point of view.

The dual hypothesis which follows from the above and which underlies the randomised clinical trial reported in this thesis, is that Doppler ultrasonography can potentially be used as a reassurance test in suspected intrauterine growth retardation to reduce costs, without this having a detrimental effect on perinatal outcome. This hypothesis was tested by comparing a management policy of suspected IUGR based on Doppler ultrasound measurements in the intervention group, with the routine management policy in the control group. The content of the thesis is as follows. Chapter 2 sketches the background literature on growth, intrauterine growth retardation, and its consequences. Chapter 3 describes the design of the randomised clinical trial (RCT) proposed here. The main results of the RCT are presented in chapter 4. Secondary findings on the relations between the Doppler ultrasound results and the outcome parameters are shown in chapter 5 . In chapter 6, the results are discussed and conclusions are drawn. Chapter 7 outlines the results of a questionnaire amongst obstetricians in the Dutch university hospitals on the present routine clinical management of suspected intrauterine growth retardation and the application of Doppler ultrasonography. Results of the questionnaire were used in the design of the study protocol. 


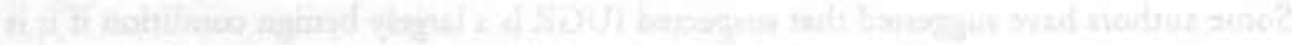

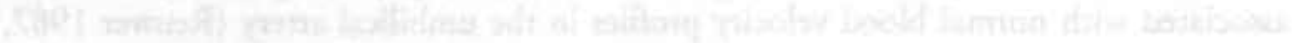

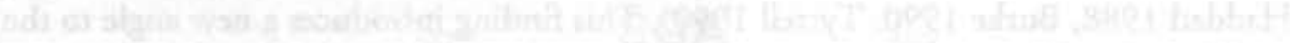

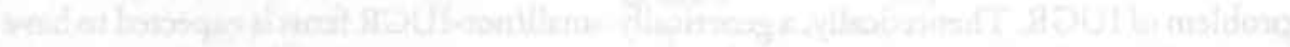

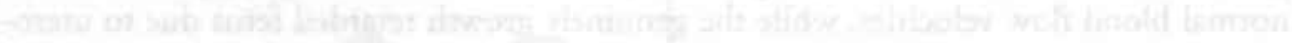

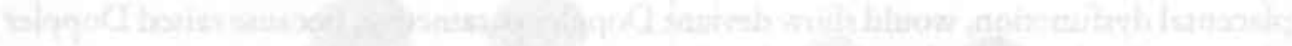

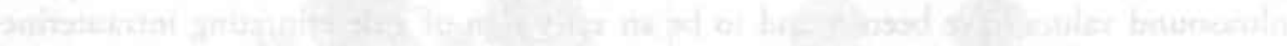

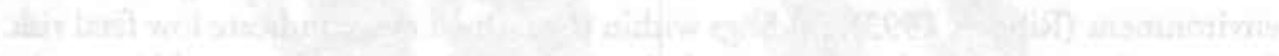

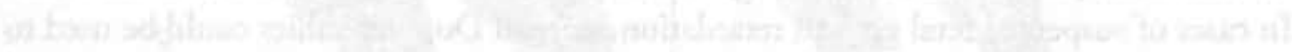

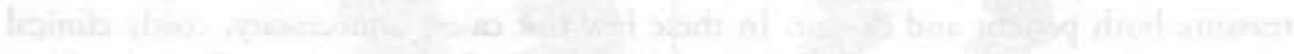

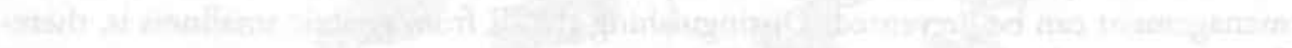

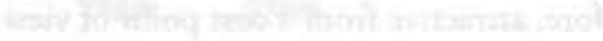

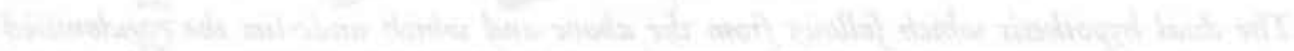

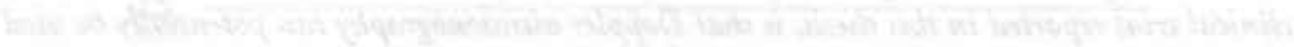

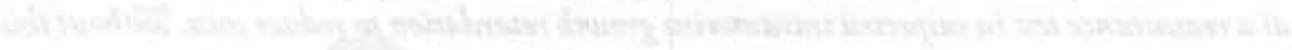

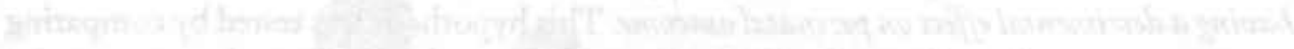

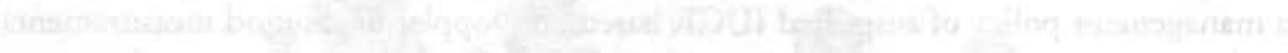

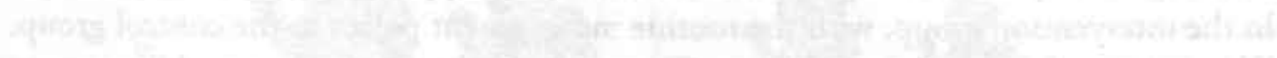

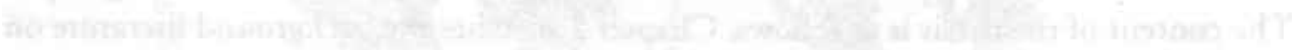

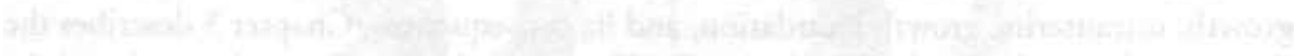

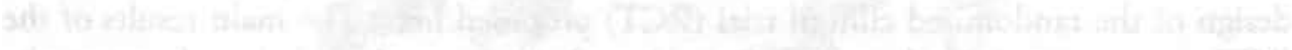

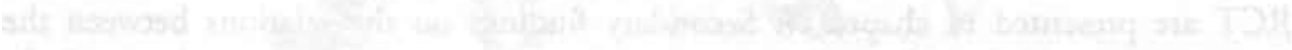

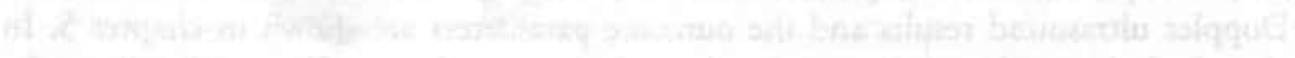

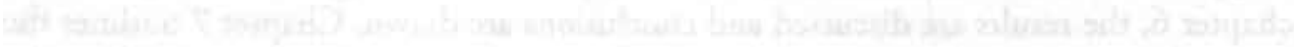

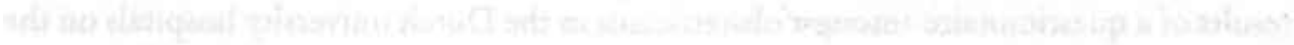

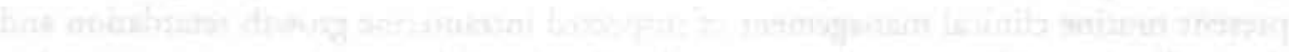

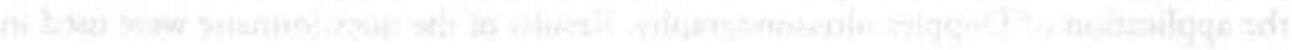

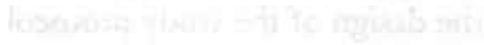

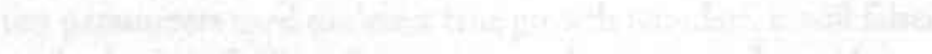

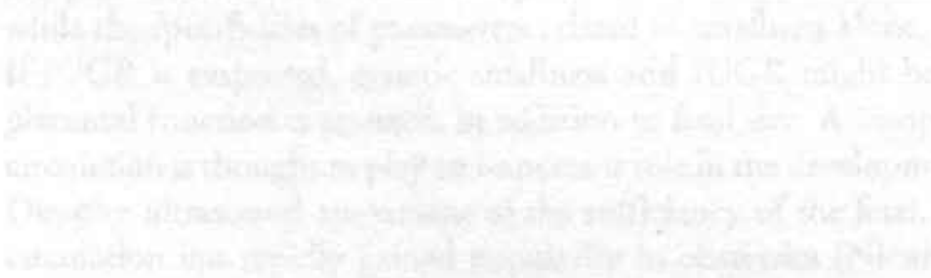

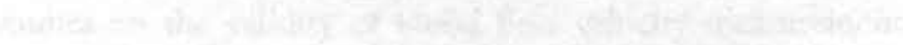




\section{Chapter 2}

\section{Review of the literature:}

\section{Growth, intrauterine growth retardation, and its} consequences

In order to place the randomised clinical trial described in this thesis into its context, the relevant literature is reviewed. Normal intrauterine growth and growth during infancy is described. With regard to intrauterine growth retardation: definition, aetiology, diagnostic tests, and consequences are reviewed. The objective of the randomised clinical trial which follows from the review, is stated at the end of this chapter.

\subsection{Normal growth}

Before intrauterine growth retardation can be discussed, optimal unconstrained growth should be defined. Anthropometric data have been published on intrauterine growth and growth during infancy (table 2.1).

'Fetal growth' has been studied cross-sectionally by anthropometric measurements of fetuses after (spontaneous) abortion or intrauterine fetal death (Potter 1975, Keen 1988, Chambers 1993), and of viable (preterm) neonates (Lubchenco 1963, Kloosterman 1970, Wälli 1980, Rooth 1985, Secher 1986a, Bossi 1987, Yudkin 1987, Lawrence 1989, Voorhorst 1990). Such anthropometric data are plotted against gestational age and referred to as 'fetal growth' curves. Several methodological problems are inherent in using such reference curves (Goldenberg 1989). Firstly, these 'fetal growth' curves do not reflect growth, but size for the gestational age ${ }^{1}(\mathrm{GA})$. Moreover, it is highly probable that intrauterine growth is not optimal in pregnancies which end in intrauterine fetal death or preterm birth. In addition, the number of contributing cases is not equal at each reference point. For instance, few preterms are born alive at the very early gestational ages and those who are, have a higher chance of having suffered an adverse intrauterine environment. Using such reference curves to assess growth will underestimate the incidence of intrauterine growth retardation.

1 Gestational age is the duration of pregnancy starting at the 1 st day of the last menstrual period. It is therefore equal to menstrual age, and 14 days longer than the conceptional age. 
Table 2.1

Anthropometric reference data in the literature

\begin{tabular}{|c|c|c|c|c|c|c|c|}
\hline Reference & Location & Period & Study & $\begin{array}{l}\text { Boys } \\
\text { n }\end{array}$ & $\begin{array}{l}\text { Girls } \\
\mathrm{n}\end{array}$ & $\begin{array}{l}\text { Both sexes } \\
n\end{array}$ & $\begin{array}{l}\text { Para- } \\
\text { meter }\end{array}$ \\
\hline Potter 1975 & USA & - & PM & - & - & $21-43$ & $h, w$ \\
\hline Keen 1988 & Great Britain & $1984-1985$ & PM & $30-78$ & $30-78$ & - & $\mathrm{h}, \mathrm{hc}, \mathrm{w}$ \\
\hline Chambers 1993 & Australia & $1987-1990$ & PM & - & - & $13-36$ & h,hc,w \\
\hline Wälli 1980 & Switzerland & $1969-1974$ & $\mathrm{NEO}$ & $4-710$ & $5-705$ & - & $\mathbf{h}, \mathrm{hc}, \mathrm{w}$ \\
\hline Rooth 1985 & Sweden & 1983 & NEO & - & - & $152-1972$ & $\mathbf{w}$ \\
\hline Secher $1986 a$ & Denmark & $1981-1983$ & NEO & $2-585$ & $1-521$ & - & $\mathbf{w}$ \\
\hline Bossi 1987 & Italy & $1973-1981$ & NEO & total 8413 & total 7923 & - & h,he \\
\hline Yudkin 1987 & Great Britain & $1978-1984$ & NEO & $89-2988$ & $84-2902$ & - & $h, w$ \\
\hline Lawrence 1989 & Sweden & $1977-1981$ & $\mathrm{NEO}$ & $125-59251$ & $107-58605$ & - & h,w \\
\hline Voorhorst 1990 & The Netherlands & $1972-1982$ & NEO & $59-1141$ & $51-1086$ & - & $\mathbf{w}$ \\
\hline Karlberg 1968 & Sweden & $1955-1960$ & $\mathrm{~L}$ & 122 & 90 & - & h,hc,w \\
\hline Largo 1980 & Switzerland & $1974-1980$ & $\mathrm{~L}$ & 74 & 54 & 一 & $h, h c, w$ \\
\hline Prader 1988 & Switzerland & $1954-1976$ & $\mathrm{~L}$ & 206 & 207 & - & $h, h c, w$ \\
\hline Guo 1990 & Canada & $1977-1978$ & $\mathrm{~L}$ & 180 & 171 & - & $h, h c, w$ \\
\hline Corrinovis 1993 & Italy & 1993 & $\mathrm{~L}$ & 5553 & 4861 & - & h,hc,w \\
\hline Roede 198.5 & The Netherlands & $1978-1980$ & C-S & 168 & 158 & - & h,hc,w \\
\hline
\end{tabular}

Period $=$ study period; Study $=$ study method: $\mathrm{PM}=$ post mortem, $\mathrm{NEO}=$ cross-sectional measurement of neonates at birth, $\mathrm{L}=$ longitudinal, $\mathrm{C}-\mathrm{S}=$ cross-sectional; Boys $/ \mathrm{Girl} / \mathrm{Both}$ sexes $=$ number of boys, girls, or fetuses of both sexes combined, in cross-sectional studies the range of fetuses or infants measured over the different of gestational ages is shown; Parameter $=$ anthropometric parameters: $h=$ height, $h c=$ head circumference, $w=$ weight; $-=$ not stated

Separate data for parity were published (Kloosterman 1970, Voorhorst 1990) which show that firstborns are smaller than later siblings. Therefore, some authors have suggested that firstborns may have experienced a less optimal intrauterine environment (Goldenberg 1989) and should possibly be excluded in a reference of optimal growth (Keirse 1981).

Longitudinal studies of postnatal growth have concentrated on either preterm (Largo 1980) or term infants (Karlberg 1968, Prader 1988, Guo 1990, Cortinovis 1993). Reference curves of neonates after uneventful pregnancies start at term by definition. Therefore, such curves do not provide a reference for early postnatal growth of preterm infants. Not all studies started measurements shortly after delivery. Some studies excluded cases associated with complications of pregnancy or neonatal disorders, in order to obtain a more optimal reference population (Lawrence 1989). For this reason, another study excluded neonates with birth weights below 2500 grams at term (Guo 
1991). This is questionable, as healthy, generically small neonates are also excluded. Finally, not all studies assessed height, head circumference, and weight. Some authors have tried to bridge the gap between 'fetal growth' and postnatal growth by joining 'fetal growth' curves to postnatal growth curves (Largo 1980, Versluys 1980). However, these curves do not join at term.

With regard to the published Dutch studies, birth weight distributions have been recently reassessed (Voorhorst 1990), and were found to be comparable to those assessed in the sixties (Kloosterman 1970). Height and head circumference (HC) were not assessed. Body proportions of Dutch children from birth to 19 years of age have been investigated cross-sectionally (Gerver 1988). A widely used reference curve in the Netherlands was constructed from a nationwide, cross-sectional anthropometric study of infants of both sexes from two weeks after delivery, onwards (Roede 1985).

From the published anthropometric studies, a selection was made for further analysis (table 2.1) on the basis of the following criteria: 'healthy' Caucasian population, known gestational age, and numeric data provided in the paper.

In order to obtain an indication of the shape of the relation between length, head circumference, and weight over time, the anthropometric data of the reviewed studies in table 2.1 were plotted against age (figures 2.1 to 2.3). Each mark in the figure represents a mean value of height, $\mathrm{HC}$, or weight obtained from one of the reviewed studies at a certain age. The mean values shown are for boys only. Conception and the expected date of delivery at 280 days are indicated.

Examination of the reviewed studies and figures 2.1 to 2.3, allow the shape of the relation of anthropometric parameters with age to be described as follows.

Post mortem height and head circumference measurements in 260 'normal' feruses with a reliable gestational age between 13 and 26 weeks (91-182 days) showed a linear relation with age (Chambers 1993).

Weight and age were exponentially related in this study. It is known that in the first and second trimester, half of the total weight gain is realised and fetal weight is doubled in the third trimester (Falkner 1985). Some 'fetal growth' curves show a decreasing weight gain from about the 36th week of pregnancy onwards (Gruenwald 1966, Largo 1980). Reduction of growth velocity is seen by some authors as a sign that the intrauterine environment has functionally reached its limit (Mendez 1985). Others dispute that placental function is limited at any time during normal pregnancy (Fox 1986). In a review of the literature (Dunn 1985), weight gain was found to increase linearly between 28 and 38 weeks of gestation. Weight gain was seen to resume this linear relation in the first 18 weeks after term delivery. In studies whereby gestational age was assessed by biparietal diameter measurements (BPD) before the 20th week of gestation, a linear relation between birth weight and gestation was found, without slowing down at term (Stembera 1985, Dunn 1985, Secher 1986a, Lindgren 1988, Kramer 1989, Wilcox 1993). These studies suggest that flattening of the weight curve before birth is due to 
16 | Chapter 2

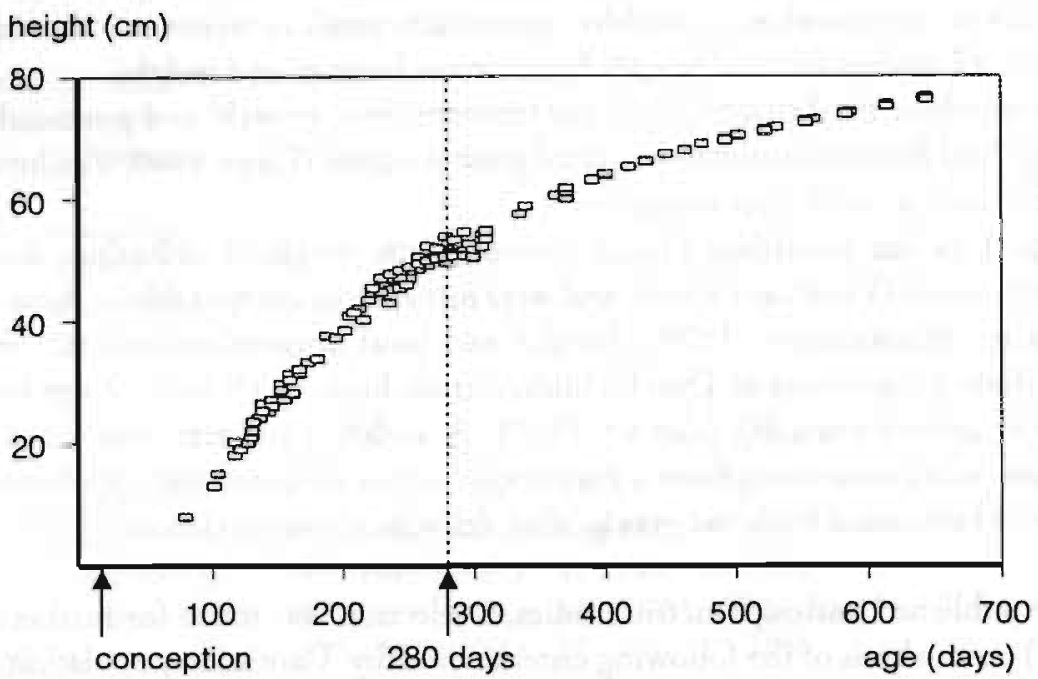

Figure 2.1

Height for age in the literature (table 2.1 , boys)

$\mathrm{HC}(\mathrm{cm})$

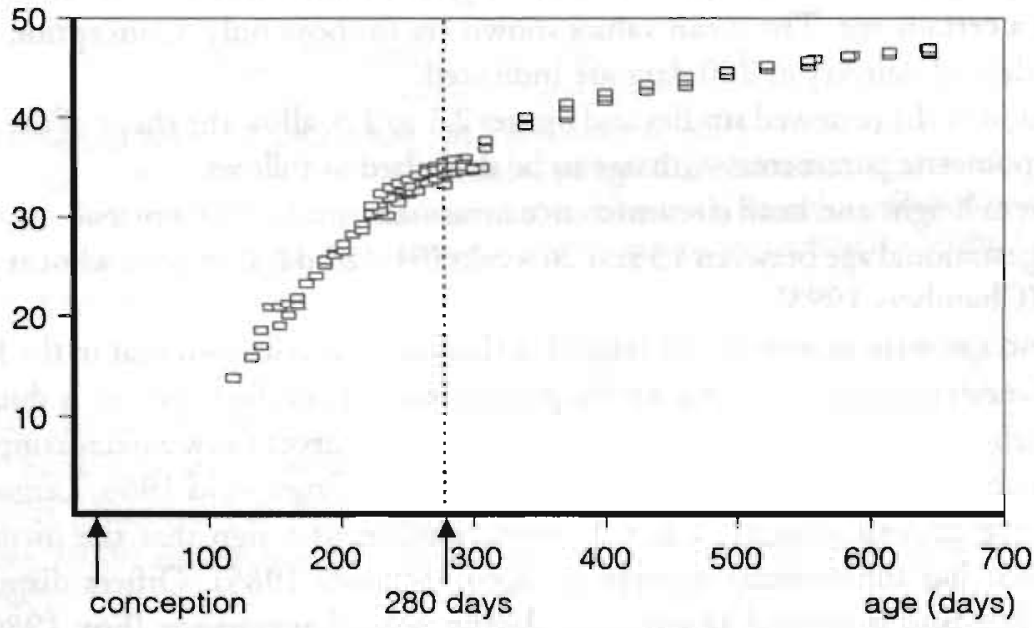

Figure 2.2

Head circumference for age in the literature (table 2.1, boys) 
weight $(\mathrm{kg})$

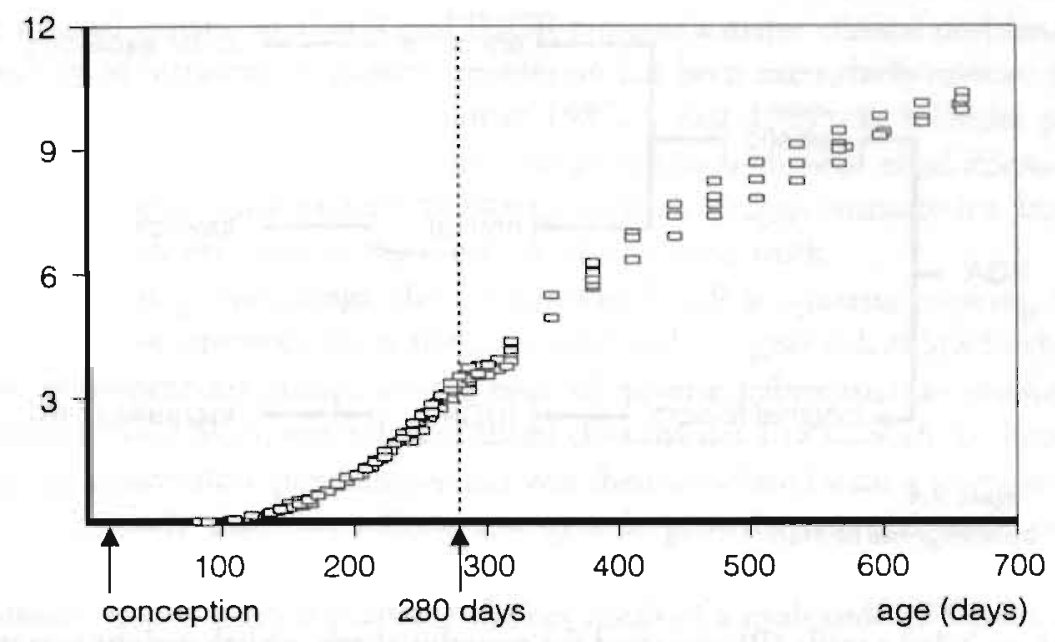

Figure 2.3

Weight for age in the literature (table 2.1, boys)

inadequate gestational age assessment and that optimal growth in this period is unrestrained in principle. Moreover, an unacceptable consequence of regarding a downward slope of the growth curve around term as physiological, would be that a growth retarded fetus whose growth is arrested may meet up with the downward sloping mean and be 'cured', if only it stays in utero long enough (Keirse 1981).

\subsection{Defining intrauterine growth retardation}

Intrauterine growth retardation (IUGR) was first recognised by the World Health Organisation as a clinical entity in 1961 (WHO 1961). Before that, a small neonate was by definition preterm.

Terms and definitions for being small are numerous. The terms dysmaturity and fetal dystrophy summarise the typical clinical appearance of the growth retarded neonate and are therefore subjective. Low birth weight (LBW) is defined as a birth weight below 2500 grams at term. The terms small for gestational age (SGA) and small or light for dates (LFD) refer to a statistical threshold value of birth weight for the gestational age in the population studied. Intrauterine or fetal malnutrition and chronic fetal distress refer to the supposed cause and timing. The terms intrauterine grouth restriction or intrauterine growth retardation (IUGR) place the emphasis on the timing of unnamed detrimental influences to growth during pregnancy.

In the various publications, the terms IUGR and SGA are often used indiscriminately. Moreover, the definitions used, differ widely ranging from a birth weight below the 


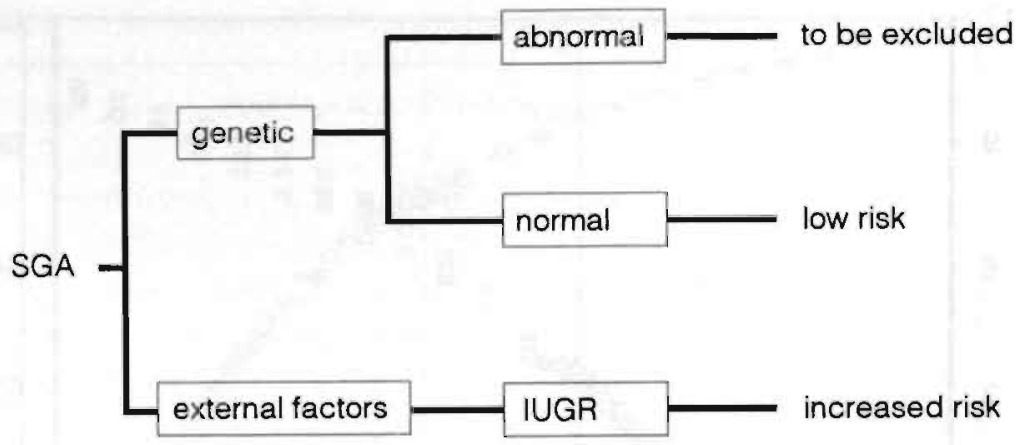

Figure 2.4

Subcategories of SGA

10th, 3rd, or 2.3rd centile (P) corrected for gestational age, a birth weight two standard deviations below the mean, to a birth weight $<2500$ grams. Such threshold values in fact define SGA. The most frequently used definition for SGA is a birth weight below the 10th centile (P10) for the gestational age.

This inability to define IUGR adequately reflects the insufficient insight in its heterogeneous pathophysiology. The lack of a gold standard or specific outcome parameters for IUGR influences all statements made in this chapter on aetiology, diagnosis, consequences, and management of IUGR (Keirse 1981).

In a population defined as SGA, three subcategories can theoretically be distinguished (figure 2.4, Wennergren 1992): deviant genetic smallness due to genetic abnormalities, normal, healthy genetic smallness, and smallness due to a restricted intrauterine environment. In the latter, the fetus cannot fulfil its genetic growth potential. This is called intrauterine growth retardation (IUGR).

Genetic abnormalities, which make up $\pm 10 \%$ of SGA and account for more than half the mortality in this group, are associated with a lower growth potential. If IUGR is the object of study, such cases should be excluded (Wennergren 1992).

\subsection{Aetiology of intrauterine growth retardation}

That growth potential is multigenetically encoded, is clear from differences in size found due to origin, sex, and parental size. The expression of growth potential depends on facilitating and restricting factors. Finally, size is a function of (gestational) age. A fetus or neonate can subsequently be small because of its genetic profile, prematurity, and/or growth retardation, each on a theoretical sliding scale of 0 to $100 \%$. With the use of early ultrasonic fetal biometry, preferably in the first trimester, the gestational age can 
be reliably assessed. However, as no definite test for IUGR exists, the differentiation between normal genetic smallness and IUGR remains a major clinical problem. The aetiology of intrauterine growth retardation has been extensively reviewed (Keirse 1981, Lin 1984, Chiswick 1985, Kramer 1987, Gross 1989). It includes placental abnormalities, maternal systemic diseases (hypertension, chronic renal disease, lupus erythematosus), intoxications (e.g. smoking, alcohol, drugs), intrauterine infections, malnutrition, malformations of the uterus, and strenuous work.

One of the most important single determinants of IUGR is cigarette smoking (Kramer 1987). Low socio-economic class is also associated with a higher risk of low birth weight. However, homogeneous groups on the basis of adverse habits such as smoking have comparable risks for SGA, regardless of social class (Miller 1985). SGA has been found to reoccur in consecutive pregnancies and was then associated with a lower mortality. It is therefore likely that this reflects low genetic growth potential and not IUGR (Bakketeig 1983).

In conclusion, size at birth is probably the net result of a multitude of factors (Wilcox 1983). A number of these factors may directly influence the quality of implantation of the blastocyst, placentation, utero-placental vascularisation, and cardiovascular and endocrinological adaptation of the mother to her pregnant state, and thus affect materno-utero-placental functioning (Duvekot 1993). Some (endocrine) factors which arise from the feto-placental unit, are in turn thought to mediate endocrinological and cardiovascular changes in the mother, which completes the circle (Longo 1984). Such, at present little understood pathophysiological mechanisms are summarised by the clinical term 'utero-placental insufficiency' and this is regarded as the most common cause of IUGR.

\subsection{Fetal growth in intrauterine growth retardation}

The effects of an insufficient intrauterine environment depend on the suddenness of onset, the severity, the duration, and the period of fetal development in which it occurs. The symmetry of fetal or neonatal body proportions is thought to reflect such factors. In several anthropometric studies comparing SGA and appropriate for gestational age infants (AGA), weight has been found to deviate most and head circumference least (Sarma 1974, Ounsted 1982a). In symmetrical or proportional growth retardation, weight, body height, and head size are equally retarded. Symmetrical growth retardation is often associated with chromosomal abnormalities. It is also thought to be the result of early onset IUGR (Villar 1982a). Differentiation from genetic smallness may be difficult if the karyotype is normal. If growth retardation occurs gradually and later in pregnancy, it results in a disproportional thin body relative to body height and head circumference. This is referred to as asymmetrical or disproportional growth retardation. 
It must be kept in mind that although such a dichotomisation of IUGR may be attractive as a tool in the study of IUGR, the occurrence of IUGR in a population is likely to result in a continuous distribution of body proportions. A parameter for neonatal proportions is Rohrer's ponderal index, which is defined as the ratio of weight of an infant to the third power of its length (Rohrer 1921).

In conclusion, 'utero-placental dysfunction' is thought to affect weight, height, and head circumference in that order, head growth being relatively 'spared'. The latter is called the brain sparing effect. However, the deficit in head circumference for the gestational age found in SGA infants is considerable (Kramer 1989). Moreover, the growth velocity of the HC has been found to be markedly increased in SGA infants in the first months after delivery in comparison to AGA infants (Davies 1980). These findings indicate that the compensatory capacity of the brain sparing effect is limited.

\subsection{Identification of the small fetus}

Accepted diagnostic tests for small fetal size are fundal height measurements and ultrasonic fetal biometry. The potential of Doppler ultrasound technology also has been studied in this respect. The accuracy with which a test identifies or predicts the outcome of interest is called the validity of a test. To assess the validity of the above tests in the detection of the small fetus, a meta-analysis was performed. For this purpose, the methods and results of the reviewed studies were summarised in a standardised fashion to facilitate comparison (tables 2.2 to 2.6).

The outcome of interest is defined by almost all reviewed studies as a birth weight for gestational age $<$ P10, which is the definition for SGA. The validity of diagnostic tests for SGA is reviewed in the following sections. The review is limited to fundal height measurements (section 2.5.1, table 2.2), ultrasonic fetal biometry (section 2.5.2, tables 2.3-2.4), and Doppler ultrasound blood flow velocity measurements in the umbilical artery (section 2.5.3, tables 2.5-2.6). The validity of these three diagnostic methods for SGA is compared at the end of section 2.5.3. The review excludes cardiotocography as the latter is generally applied as a secondary monitoring test, after a small fetus has been identified by other tests, rather than as a diagnostic test for SGA.

Published studies were only included in the review if it was possible to construct a two by two table of the test result against outcome, as shown in figure 2.5 . The definition of an abnormal test result and the definition of SGA had to be explicitly stated in the paper.

The epidemiological parameters traditionally used to quantify the diagnostic value of a test are shown in figure 2.5. The parameters sensitivity (Sens) and specificity (Spec) indicate how well a test identifies cases respectively with or without the condition of interest (SGA). The prior probability that the condition of interest is present, is given by the prevalence (Prev). The likelihood ratio of a positive test (LR+) gives the ratio 


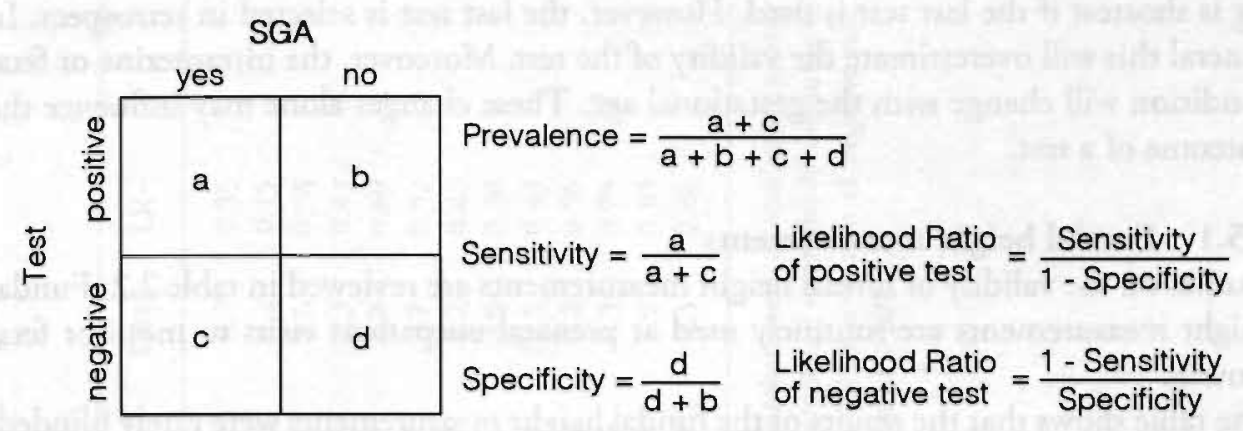

Figure 2.5

Epidemiological parameters

between the chance of a positive test result if the fetus is SGA and the chance of a positive test result if the fetus is not SGA. The likelihood ratio of a negative test (LR-) gives a similar ratio for a negative test result. A likelihood ratio of approximately 1 indicates that the test result does not improve on the prior odds. The test is more informative if the likelihood ratio of a positive test result approaches infinity and the likelihood ratio of a negative test result approaches 0.

A number of methodological catches in the interpretation of diagnostic tests has been described (Mohide 1989). Some are discussed below.

A high prevalence population has generally been preselected for further testing or monitoring. The adverse condition of interest in these cases is likely to be more severe than in the general population. Identification will then be easier and the sensitivity of the test higher. In the general population, the prevalence of SGA is about $10 \%$ by definition. Therefore, studies with a prevalence of SGA in the 'normal' range of $\pm 10 \%$ and those with high risk populations reflected by a high prevalence, were shown in separate tables.

In the literature reviewed, the selection criteria for high risk varied considerably. It is conceivable that the validity of a test for SGA could differ between pregnancies complicated by, for instance, hypertension, blood loss, or suspected growth retardation. In the assessment of the validity of a test, it is methodologically correct to keep the test results from the clinicians in charge, as the knowledge of the test result could change the management policy. If the subsequent management policy is effective, it might improve the outcome, which in turn would negatively influence the validity of the test (Grant 1984). Whether this condition was met in the reviewed studies is shown in the tables under the heading 'blind'. As concurrent testing may also influence clinical management, studies using other tests besides the test of interest were excluded. The results of the last test before delivery is used in the analysis in some studies. A test result is most representative of the clinical condition at the time of testing and the time 
lag is shortest if the last test is used. However, the last test is selected in retrospect. In general this will overestimate the validity of the test. Moreover, the intrauterine or fetal condition will change with the gestational age. These changes alone may influence the outcome of a test.

\subsubsection{Fundal height measurements}

Studies on the validity of fundal height measurements are reviewed in table 2.2. Fundal height measurements are routinely used at prenatal outpatient visits to monitor fetal growth.

The table shows that the results of the fundal height measurements were rarely blinded. The prevalence of SGA found in some of the studies is up to three times as high as the prevalence found in the general population in which fundal height measurement are generally performed. Some of these studies seem to have applied some kind of selection (Belizán 1978, Quaranta 1981). In one study, more infants with low and high birth weights for the gestational age were included into the study, in order to establish the validity of fundal height measurements more efficiently (Secher 1990).

The values for sensitivity shown in table 2.2 , show that fundal height measurements correctly identify approximately half the SGA infants. From the $\mathrm{LR}+\mathrm{it}$ can be concluded that the chance of having a positive test result in fundal height measurements is approximately six times higher in SGA than in AGA pregnancies.

A research group which has monitored the validity of fundal height measurements since the introduction of this technique into their routine clinical practice, has shown that the validity decreased due to the decreasing rigour with which the measurement technique was performed (Belizán 1990).

\subsubsection{Ultrasonic fetal biometry}

A number of studies has assessed the validity of ultrasonic fetal biometry in the identification of SGA. Separate tables were constructed for normal prevalence (table 2.3) and high prevalence populations (table 2.4).

Again, not all studies were blinded, and many did not even state whether an attempt was made to keep the results from the clinicians. Gestational age was assessed by early ultrasonic biometry in almost all studies. The biometric parameters and the threshold values used to define a positive test differed widely between the studies.

The sensitivity and the likelihood ratio of a positive test are generally somewhat higher in ultrasonic fetal biometry (tables 2.3-2.4) than in fundal height measurements (table 2.2). In conclusion, the validity of ultrasonic fetal biometry seems somewhat better than that of fundal height measurements. These differences are, however, not very impressive and the variation between the studies is large. 
Table 2.2

Validity of fundal height measurements in the detection of SGA

\begin{tabular}{|c|c|c|c|c|c|c|c|c|c|c|c|}
\hline Reference & Blind & Scan & Freq & Threshold value & SGA & $\mathrm{N}$ & Prev & Sens & Spec & $\mathrm{LR}+$ & LR- \\
\hline Westin 1977 & no & - & - & $<M-2 S D$ & $<M-1 S D$ & 428 & 11.0 & 68.1 & 92.1 & 8.7 & 0.35 \\
\hline Bclizán 1978 & yes & - & routine & $<\mathrm{P} 10$ & $<\mathrm{P} 10$ & 139 & 31.7 & 86.4 & 89.5 & 8.2 & 0.15 \\
\hline Quaranta 1981 & no & - & routine & $<\mathrm{P} 10^{1}$ & $<\mathrm{P} 10$ & 138 & 29.7 & 73.2 & 79.4 & 3.6 & 0.34 \\
\hline Wallin 1981 & no & - & routine & $<\mathrm{M}-3 \mathrm{~cm}>2 \mathrm{x}$ & $<\mathrm{M}-2 \mathrm{SD}$ & 812 & 12.0 & 61.9 & 88.3 & 5.3 & 0.43 \\
\hline Calvert 1982 & no & - & routine & $<\mathrm{P} 10^{1}$ & $<P 10$ & 381 & 11.8 & 35.6 & 93.8 & 5.7 & 0.69 \\
\hline Rosenberg 1982 & no & - & routine & $<\mathrm{P} 10^{\mathrm{I}}$ & $<\mathrm{P} 10$ & 753 & 6.6 & 56.0 & 84.6 & 3.7 & 0.52 \\
\hline Persson 1986 & no & - & routine & $<\mathrm{M}-2 \mathrm{SD}$ & $<\mathrm{P} 10^{2}$ & 2919 & 9.0 & 26.6 & 88.0 & 2.2 & 0.83 \\
\hline Pearce 1987 & กо & - & routine & $<\mathrm{P} 10$ & $<\mathrm{P} 10$ & 699 & 14.3 & 76.0 & 79.0 & 3.6 & 0.30 \\
\hline Cnattingius 1988 & no & - & routine & $<\mathrm{M}-3 \mathrm{~cm}$ & $<M-2 S D$ & 3038 & 0.8 & 58.3 & 97.0 & 19.5 & 0.43 \\
\hline Stuart 1989 & no & yes & - & $<\mathrm{P} 10$ & $<\mathrm{P} 10$ & 1139 & 7.6 & 50.6 & 87.9 & 4.2 & 0.56 \\
\hline Lindhart 1990 & no & yes & $1-3 x$ & $2 x<\mathrm{P} 10^{3}$ & $<\mathrm{P} 10$ & 804 & 7.6 & 27.9 & 96.6 & 8.3 & 0.75 \\
\hline Secher 1990 & no & yes & routine & $<\mathrm{P}_{1} 0^{1}$ & $<P 9.4$ & 199 & 29.2 & 24.1 & 95.0 & 4.9 & 0.80 \\
\hline Jensen 1991a & no & yes & routine & $\leq \mathrm{M}-2 \mathrm{~cm}$ & $<\mathrm{P} 10$ & 831 & 11.5 & 40.6 & 87.5 & 3.3 & 0.68 \\
\hline
\end{tabular}

Blind = study blinded to clinicians in charge; $S c a n=$ early ultrasonic GA assessment; Freq = testing frequency: routine $=$ measurements at routine outpatient visits; Threshold value $=$ value which distinguishes a normal from an abnormal test result; $S G A=$ definition of $S G A: M=$ mean, $P=$ centile; $N=$ number of patients, Prev = prevalence of SGA, Sens = sensitivity, Spec $=$ specificity, LR+ = likelihood ratio of positive test, LR- = likelihood ratio of negative test; - = not stated; ${ }^{1}$ at two consecutive measurements or three single measurements; ${ }^{2}$ or weight/height $<\mathrm{M}-2 \mathrm{SD} ;{ }^{3}$ or a fall of $>20 \%$ 
Table 2.3

Validity of ultrasonic feral biometry in the detection of SGA in normal prevalence populations

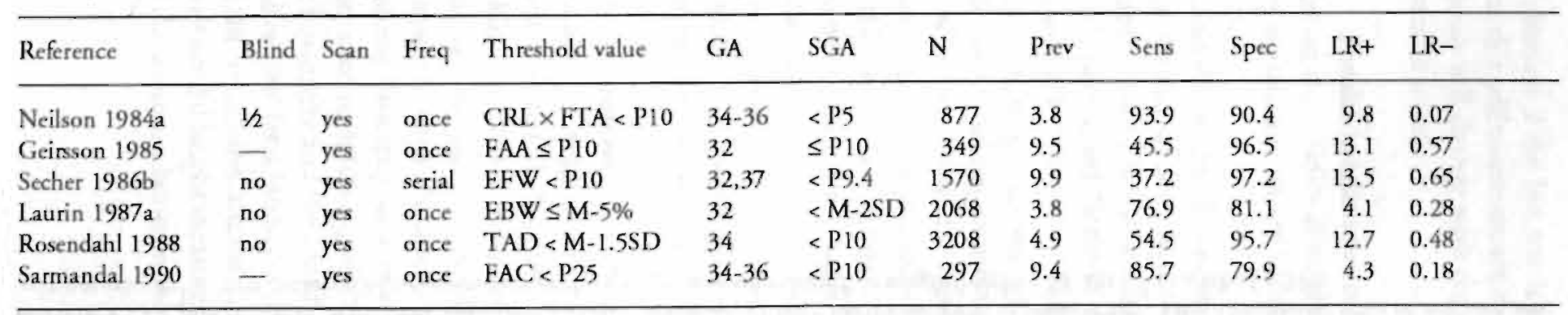

Blind = study blinded to clinicians in charge, $1 / 2=$ blind in control group; Scan = early ultrasonic GA assessment; Freq = testing frequency: once $=$ single measurement, serial $=$ repeated measurements; Threshold value $=$ value which distinguishes a normal from an abnormal rest result: $\mathrm{HC}=$ head circumference, $\mathrm{CRL}=$ crown rump length, FTA $=$ fetal thoracic area, $\mathrm{FAA}=$ fetal abdominal area, FAC $=$ fetal abdominal circumference, TAD $=$ transverse abdominal diameter, EFW = estimated fetal weight, EBW = estimated birth weight; $\mathrm{GA}=\mathrm{GA}$ in wecks at testing: last test = result of last test before delivery used for analysis; $\mathrm{SGA}=$ definition of SGA: $\mathrm{P}=$ birth weight centile, $\mathrm{M}=$ mean, $\mathrm{SD}=$ standard deviation, $\mathrm{SDS}=$ standard deviation score; $\mathrm{N}=$ size of popularion, Prev $=$ prevalence of SGA, Sens $=$ sensirivity, Spec $=$ specificity, LR+ $=$ likelihood ratio of positive test, LR- $=$ likelihood ratio of negative test; $-\approx$ not stated

Table 2.4

Validity of ultrasonic fetal biometry in the detection of SGA in high prevalence populations

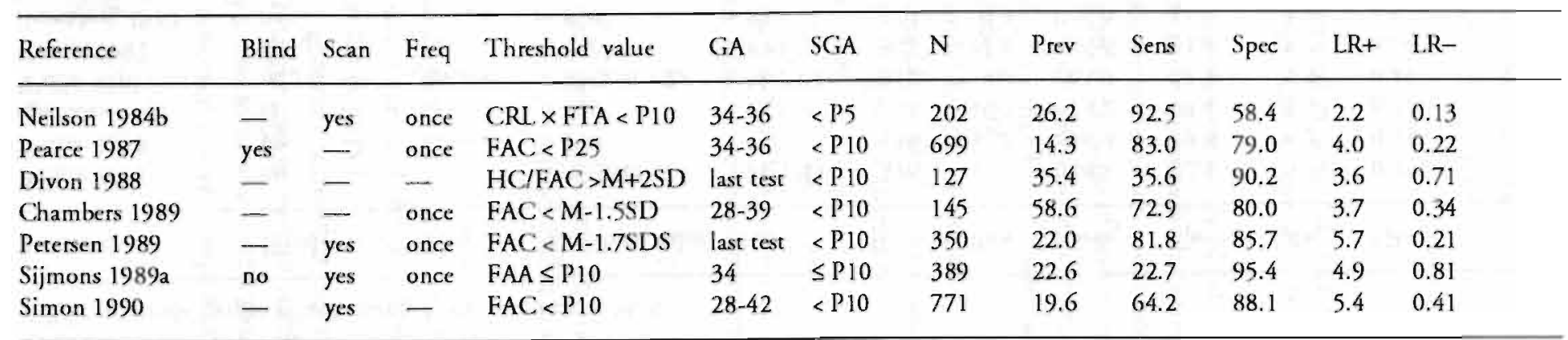

sce leecend rable 2.3 
Table 2.5

Validity of umbilical artery Doppler ultrasound measurements in the detection of SGA in normal prevalence populations

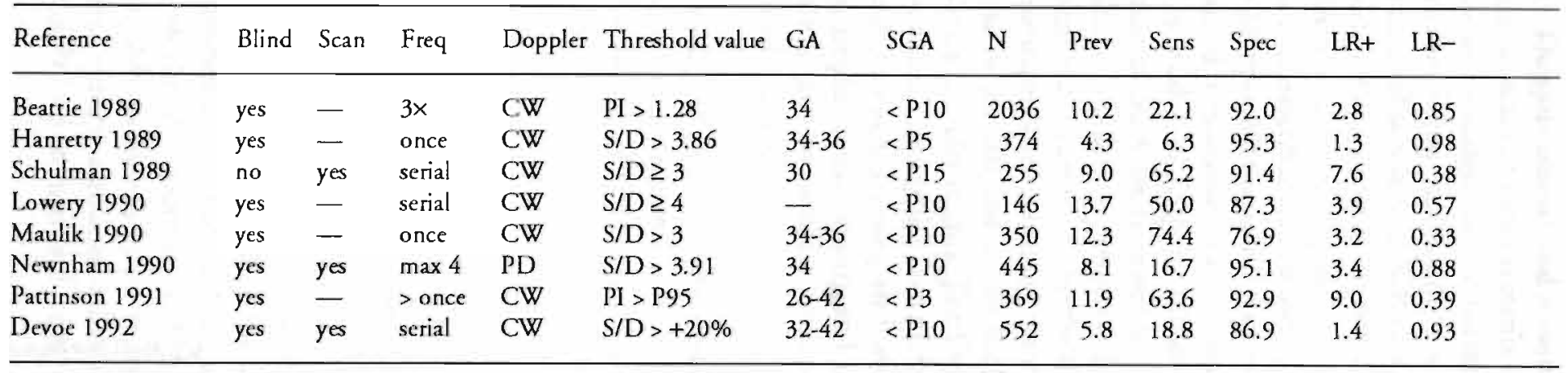

Blind = study blinded to clinicians in charge; Scan = early ultrasonic GA assessment; Freq = testing frequency: once = single measurement, serial = repeated measurements; Doppler $=$ Doppler technique: $\mathrm{PD}=$ pulsed Doppler, $\mathrm{CW}=$ continuous wave; Threshold value $=$ value which distinguishes a normal from an abnormal test result: $\mathrm{P}=$ centile, $\mathrm{PI}=$ Pulsatility Index, $S / D=$ systolic-diastolic ratio (A/B ratio); $\mathrm{GA}=\mathrm{GA}$ in weeks at testing: last test = result of last test before delivery used for analysis; $\mathrm{SGA}=$ definition of $\mathrm{SGA}: \mathrm{P}=$ birth weight centile, $\mathrm{M}=$ mean, $\mathrm{SD}=$ standard deviation; $\mathrm{N}=$ size of population, Prev = prevalence of SGA, Sens = sensitivity, Spec = specificity, LRt = likelihood ratio of positive test, LR- = likelihood ratio of negative test; $-=$ not stated 
Table 2.6

Validity of umbilical artery Doppler ultrasound measurements in the detection of SGA in high prevalence populations

\begin{tabular}{|c|c|c|c|c|c|c|c|c|c|c|c|c|c|}
\hline Reference & Blind & Scan & Freq & Doppler & Threshold value & GA & $S G A$ & $N$ & Prev & Sens & Spec & LRt & LR- \\
\hline Fleisher 1985 & - & no & $\geq 2$ & $C W$ & $S / D \geq 3$ & $31-39$ & $<\mathrm{P} 10$ & 137 & 16.8 & 78.3 & 83.3 & 4.7 & 0.26 \\
\hline Berkowitz 1988 & yes & yes & serial & CW & $\mathrm{S} / \mathrm{D}>3$ & $30-42$ & $<\mathrm{PlO}$ & 172 & 25.0 & 48.8 & 86.8 & 3.7 & 0.59 \\
\hline Detnpster 1988 & yes & - & - & CW & $\mathrm{S} / \mathrm{D})>\mathrm{P} 97$ & last test & $<\mathrm{P} 10$ & 205 & 40.0 & 37.8 & 84.6 & 2.5 & 0.74 \\
\hline Divon 1988 & - & yes & - & $C W$ & $S / D>3$ & last test & $<\mathrm{P} 10$ & 127 & 35.4 & 48.9 & 93.9 & 8.0 & 0.54 \\
\hline Jensen 1991b & yes & yes & - & $\mathrm{PD}$ & $S / D>3$ & last test & $<P 5$ & 94 & 29.8 & 71.4 & 77.3 & 3.1 & 0.37 \\
\hline Ott 1991 & - & - & - & $\mathrm{PD}$ & $S / D \geq 3$ & last test & $<P 10$ & 149 & 32.2 & 60.4 & 74.3 & 2.4 & 0.53 \\
\hline Trudinger 1991 & - & - & $>$ once & $C W$ & $\mathrm{~S} / \mathrm{D}>\mathrm{P} 95$ & last test & $<P 10$ & 2178 & 27.0 & 50.2 & 85.4 & 3.4 & 0.58 \\
\hline Miller 1992 & - & $y e s$ & - & $\mathrm{PD}$ & $S / D \geq 3$ & last test & $<P 10$ & 136 & 33.8 & 67.4 & 75.6 & 2.8 & 0.43 \\
\hline Theron 1992 & yes & yes & - & CW & $\mathrm{PI}>\mathrm{P95}$ & - & $<\mathrm{P} 10$ & 121 & 47.9 & 46.6 & 90.5 & 4.9 & 0.59 \\
\hline Soothill 1993 & no & - & $>$ once & $C W$ & $\mathrm{Pl}>\mathrm{P97.5}$ & last test & $<P 2.5$ & 191 & 15.7 & 20.0 & 96.3 & 5.4 & 0.83 \\
\hline
\end{tabular}

see legend table 2.5 


\subsubsection{Doppler ultrasound measurements in the umbilical artery}

Doppler ultrasound measurements of the blood velocity profiles in the umbilical artery provide some insight in the haemodynamic condition of the fetus. Abnormal flow patterns are found significantly more often in SGA than in AGA fetuses. This effect could be attributable to IUGR to a large degree. In the literature, Doppler techniques are used, both to detect SGA (tables 2.5, 2.6) and to monitor fetuses suspected of being SGA by the other techniques.

As the trial described in this thesis, focuses on the potential clinical value of umbilical artery Doppler ultrasound measurements, the effectivity and the technique of Doppler ultrasound measurements are discussed at greater length in sections 2.6 and 3.2.

Validiry studies of Doppler ultrasound measurements in the umbilical artery in the detection of SGA are reviewed in tables 2.5 and 2.6 for normal prevalence and high prevalence populations, respectively. Again differences in methodology are apparent. Different indices are used, threshold values used to define an abnormal test result differ, and participants are measured either once or serially, and at different gestational ages. The use of the last test in the analysis may have biased results in some of the high prevalence Doppler studies. The Doppler studies in high prevalence populations tended to have a smaller study size than studies on fundal height measurements or fetal biometry. The tables show that, compared with accepted diagnostic techniques for SGA, such as fundal height measurements and ultrasonic fetal biometry, the validity of Doppler ultrasound measurements is comparable or lower. Less than half the SGA infants are identified prenatally.

The validity of fundal height measurements, ultrasonic fetal biometry, and Doppler ultrasound measurements in the umbilical artery is reviewed in tables 2.2 to 2.6 . Notable are the differences in design between the studies. Prevalence, inclusion criteria, threshold values used to define a positive test result, and definitions of outcome differed widely. Therefore, a formal mera-analysis of the different studies could nor be performed. Another major problem was that in many of the studies, the test results were not blinded or that blinding was not stated explicitly.

By plotting the test properties for identifying or excluding SGA (not shown), it could be seen that they deviated little between fundal height measurements, ultrasonic fetal biometry, and Doppler ultrasound measurements. The Doppler studies generally had a somewhat smaller study population. The specificity was comparable in all three tests. The sensitivity and the likelihood ratio of a positive test were somewhat higher for ultrasonic fetal biometry than for fundal height measurements or Doppler ultrasound measurements. In conclusion, the tables show that the validity of Doppler ultrasonography in the identification of SGA did nor differ much from fundal height measurements, and was slightly below that of ultrasonic fetal biometry.

All cases wrongly identified will cause unnecessary worry to the parents concerned, and potentially lead to increased frequency of outpatient visits, fetal monitoring, and even 
prenatal hospitalisation. This overtreatment is costly. A test that reduces the number of wrongly identified cases, i.e. can differentiate between IUGR and genetic smallness, is potentially attractive, if only from a costs point of view.

\subsection{Effectiveness of Doppler ultrasound measurements: Randomised Clinical Trials}

Even if there is a perfectly valid test for SGA (or even growth retardation), its application does not necessarily improve patient management and/or perinatal outcome. After a diagnostic test's validity has been established and found to be adequate, the next question is whether the clinical application of the test is of genuine benefit to patients. The most informative study design with which this can be investigated, is a randomised clinical trial. After dividing eligible patients at random over the two study groups, the test (and the associated management policy) is applied to the intervention group, while the control group receives the conventional clinical management.

A meta-analysis of 10 published randomised clinical trials (RCT) on the clinical application of Doppler ultrasound measurements, is presented in tables 2.7 to 2.12 . In the RCT of Johnstone (1993), a separate analysis was preplanned for a subgroup of cases with suspected IUGR and/or hypertension. These results are presented first in the tables, followed by the results of the whole study group. Two RCTs are not presented in the tables, but are discussed in the text below the tables.

The customary outcomes presented are elective delivery (table 2.7), emergency Caesarean secrion (CS, table 2.8), perinatal mortality (table 2.9), SGA (table 2.10), Apgar score at five minutes (table 2.11), and admission to the neonatal care unit (table 2.12 ). In the tables, the occurrence of an outcome parameter expressed as proportion and as percentage, is shown for the intervention group and for the control group. Study objectives, study design, and results of these and two other RCTs are discussed separately for each trial in the text below the tables. Not all outcome measures presented in the tables were also stated as main objective of the trial concerned.

The graphic display of the odds ratio (OR) and the $95 \%$ confidence interval $(95 \% \mathrm{CI})$ in the tables offers a rapid visual meta-analysis of the effectiveness of the clinical application of Doppler ultrasound measurements in each trial (Chalmers 1989). The odds ratio is the comparison of the odds of the outcome in the intervention group with the odds in the control group in a randomised clinical trial design. For instance, an OR of 0.30 indicates that the occurrence risk of the condition of interest in the intervention group is reduced by $70 \%$ in comparison to the control group. The $95 \% \mathrm{CI}$ of the OR indicates the range of the true reduction. If the $95 \% \mathrm{CI}$ includes ' 1 ', the reduction in adverse outcome between the intervention and the control group is not significant. Note that the ORs and the $95 \% \mathrm{CIs}$ are plotted on a logarithmic scale. 
Table 2.7

Randomised clinical trials

Effectiveness of Doppler ultrasound measurements: Elective delivery ${ }^{1}$

\begin{tabular}{|c|c|c|c|c|c|c|c|c|c|c|}
\hline \multirow[t]{2}{*}{ Reference } & \multicolumn{2}{|c|}{ Intervention group } & \multicolumn{2}{|c|}{ Control group } & \multicolumn{2}{|c|}{ Odds ratio } & \multicolumn{4}{|c|}{$\begin{array}{l}\text { Odds ratio and } 95 \% \\
\text { confidence interval }\end{array}$} \\
\hline & $\mathrm{n}$ & $\%$ & $\mathrm{n}$ & $\%$ & & $95 \% \mathrm{CI}$ & 0.01 & 0.1 & 1 & 10 \\
\hline Trudinger 1987 & $82 / 127$ & 64.6 & $107 / 162$ & 66.1 & 0.94 & $0.58-1.53$ & & & & \\
\hline McParland 1988 & $138 / 254$ & 54.3 & $156 / 255$ & 61.2 & 0.76 & $0.53-1.07$ & & & & \\
\hline Tyrrell 1990 & $92 / 230$ & 40.0 & $91 / 222$ & 41.0 & 0.96 & $0.67-1.42$ & & & & \\
\hline Omtzigt 1990 & $168 / 809$ & 20.8 & $148 / 789$ & 18.8 & 1.13 & $0.89-1.45$ & & & & \\
\hline Newnham 1991 & $125 / 254$ & 49.2 & $137 / 251$ & 54.6 & 0.81 & $0.57-1.14$ & & & & \\
\hline Hofmeyr 1991 & $111 / 438$ & 25.3 & $109 / 459$ & 23.8 & 1.09 & $0.80-1.48$ & & & & \\
\hline Ålmström 1992 & $59 / 214$ & 27.6 & $68 / 212$ & 32.1 & 0.81 & $0.53-1.22$ & & & & \\
\hline Davies 1992 & $356 / 1246$ & 28.6 & $355 / 1229$ & 28.9 & 0.98 & $0.83-1.17$ & & & & \\
\hline Mason 1993 & $209 / 1015$ & 20.6 & $213 / 1001$ & 21.3 & 0.96 & $0.77-1.19$ & & & & \\
\hline Johnstone 1993 & $180 / 370$ & 48.7 & $194 / 384$ & 50.5 & 0.93 & $0.70-1.23$ & & & & \\
\hline Johnstone 1993 & $397 / 1114$ & 35.6 & $444 / 1175$ & 37.8 & 0.91 & $0.77-1.08$ & & & d & \\
\hline
\end{tabular}

${ }^{1}$ is defined as induction of labour or elecrive Caesarean secrion (CS)

\section{Table 2.8}

Randomised clinical trials

Effectiveness of Doppler ultrasound measurements: Emergency Caesarean section

\begin{tabular}{|c|c|c|c|c|c|c|c|c|c|c|}
\hline \multirow[t]{2}{*}{ Reference } & \multicolumn{2}{|c|}{ Intervention group } & \multicolumn{2}{|c|}{ Control group } & \multicolumn{2}{|c|}{ Odds ratio } & \multicolumn{3}{|c|}{$\begin{array}{l}\text { Odds ratio and } 95 \% \\
\text { confidence interval }\end{array}$} & \multirow[b]{2}{*}{10} \\
\hline & $\mathrm{n}$ & $\%$ & $\mathrm{n}$ & $\%$ & & $95 \% \mathrm{Cl}$ & 0.01 & 0.1 & 1 & \\
\hline Trudinger 1987 & $13 / 127$ & 10.2 & $31 / 162$ & 19.1 & 0.50 & $0.26-0.96$ & & & & \\
\hline McParland 1988 & not stated & & & & & & & & & \\
\hline Tyrretl 1990 & not stated & & & & & & & & & \\
\hline Omtzigt 1990 & $78 / 809$ & 9.6 & $85 / 789$ & 10.8 & 0.88 & $0.64-1.22$ & & & & \\
\hline Newnham 1991 & $65 / 254$ & 25.6 & $53 / 251$ & 21.1 & 1.28 & $0.85-1.94$ & & & & \\
\hline Hofmeyr 1991 & $63 / 438$ & 14.4 & $90 / 459$ & 19.6 & 0.69 & $0.49-0.98$ & & & & \\
\hline Ålmström 1.992 & $21 / 214$ & 9.8 & $40 / 212$ & 18.9 & 0.48 & $0.28-0.82$ & & & & \\
\hline Davies 1992 & $13 / 1246$ & 1.0 & $11 / 1229$ & 0.9 & 1.17 & $0.52-2.61$ & & & & \\
\hline Mason 1993 & not stated & & & & & & & & & \\
\hline Johnstone 1993 & $51 / 370$ & 13.8 & $51 / 384$ & 13.3 & 1.04 & $0.69-1.58$ & & & & \\
\hline Johnstone 1993 & $117 / 1114$ & 10.5 & $125 / 1175$ & 10.6 & 0.99 & $0.76-1.29$ & & & & \\
\hline
\end{tabular}


Table 2.9

Randomised clinical trials

Effectiveness of Doppler ultrasound measurements: Perinatal mortality ${ }^{1}$

\begin{tabular}{|c|c|c|c|c|c|c|c|c|c|c|}
\hline \multirow[t]{2}{*}{ Reference } & \multicolumn{2}{|c|}{ Intervention group } & \multicolumn{2}{|c|}{ Control group } & \multicolumn{2}{|c|}{ Odds ratio } & \multicolumn{3}{|c|}{$\begin{array}{l}\text { Odds ratio and } 95 \% \\
\text { confidence interval }\end{array}$} & \multirow[b]{2}{*}{10} \\
\hline & $\mathrm{n}$ & $\%$ & $\mathrm{n}$ & $\%$ & & $95 \% \mathrm{Cl}$ & 0.01 & 0.1 & 1 & \\
\hline Trudinger 1987 & $0 / 127$ & 0.0 & $2 / 162$ & 1.2 & 0.17 & $0.01-2.74$ & & & & \\
\hline McParland 1988 & $6 / 254$ & 2.4 & $20 / 255$ & 7.8 & 0.32 & $0.15-0.71$ & & & & \\
\hline Tyrrell 1990 & $1 / 250$ & 0.4 & $2 / 250$ & 0.8 & 0.51 & $0.05-4.95$ & & & & \\
\hline Omtzigt 1990 & $13 / 825$ & 1.6 & $22 / 815$ & 2.7 & 0.58 & $0.30-1.14$ & & & & \\
\hline Newnham 1991 & $7 / 275$ & 2.6 & $7 / 270$ & 2.6 & 0.98 & $0.34-2.83$ & & & & \\
\hline Hofmeyr 1991 & $3 / 438$ & 0.7 & $8 / 459$ & 1.7 & 0.42 & $0.13-1.37$ & & & & \\
\hline Almström 1992 & $0 / 214$ & 0.0 & $2 / 212$ & 0.9 & 0.13 & $0.01-2.14$ & & & & \\
\hline Davies 1992 & $16 / 1246$ & 1.3 & $4 / 1229$ & 0.3 & 3.31 & $1.37-7.97$ & & & & \\
\hline Mason 1993 & $4 / 1015$ & 0.4 & $5 / 1001$ & 0.5 & 0.79 & $0.21-2.92$ & & & & \\
\hline Johnstone 1993 & not stated & & & & & & & & & \\
\hline Johnstone 1993 & $8 / 1114$ & 0.7 & $10 / 1175$ & 0.9 & 0.84 & $0.33-2.13$ & & & & \\
\hline
\end{tabular}

${ }^{1}$ cases with chromosomal and/or congenital defects were excluded

Table 2.10

Randomised clinical trials

Effectiveness of Doppler ultrasound measurements: SGA (birth weight $<$ P10) ${ }^{1}$

\begin{tabular}{|c|c|c|c|c|c|c|c|c|c|c|}
\hline \multirow[t]{2}{*}{ Reference } & \multicolumn{2}{|c|}{ Intervention group } & \multicolumn{2}{|c|}{ Control group } & \multicolumn{2}{|c|}{ Odds ratio } & \multicolumn{3}{|c|}{$\begin{array}{l}\text { Odds ratio and } 95 \% \\
\text { confidence interval }\end{array}$} & \multirow[b]{2}{*}{10} \\
\hline & $\mathrm{n}$ & $\%$ & $n$ & $9 \%$ & & $95 \% \mathrm{CI}$ & 0.01 & 0.1 & 1 & \\
\hline Trudinger 1987 & $23 / 127$ & 18.1 & $18 / 162$ & 11.1 & 1.77 & $0.91-3.45$ & & & & \\
\hline McParland 1988 & $182 / 254$ & 71.7 & $181 / 255$ & 71.0 & 1.03 & $0.70-1.52$ & & & & \\
\hline Tyrrell 1990 & not stated & & & & & & & & & \\
\hline Orntzigt 1990 & $115 / 825$ & 13.9 & $111 / 815$ & 13.6 & 1.03 & $0.78-1.36$ & & & & \\
\hline Newnham 1991 & $93 / 275$ & 33.8 & $89 / 270$ & 33.0 & 1.04 & $0.73-1.48$ & & & & \\
\hline Hofneyr 1991 & not stated & & & & & & & & & \\
\hline Ålmström 1992 & not stated & & & & & & & & & \\
\hline Davies 1992 & $125 / 1246$ & 10.0 & $127 / 1229$ & 10.3 & 0.97 & $0.75-1.26$ & & & 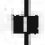 & \\
\hline Mason 1993 & not stated & & & & & & & & & \\
\hline Johnstone 1993 & not stated & & & & & & & & & \\
\hline Johnstone $1993^{2}$ & $122 / 1095$ & 11.1 & $122 / 1147$ & 10.6 & 1.05 & $0.81-1.37$ & & & & \\
\hline
\end{tabular}

1 cases with chromosomal and/or congenital defects were excluded

2 singletons only, unclear if cases with chromosomal and/or congenital defects had been excluded 
Table 2.11

Randomised clinical trials

Effectiveness of Doppler ultrasound measurements: Apgar score at 5 minutes less than 7

\begin{tabular}{|c|c|c|c|c|c|c|c|c|}
\hline \multirow[t]{2}{*}{ Reference } & \multicolumn{2}{|c|}{ Intervention group } & \multicolumn{2}{|c|}{ Control group } & \multicolumn{2}{|c|}{ Odds ratio } & \multicolumn{2}{|c|}{$\begin{array}{l}\text { Odds ratio and } 95 \% \\
\text { confidence interval }\end{array}$} \\
\hline & $n$ & $\%$ & $\mathrm{n}$ & $\%$ & & $95 \% \mathrm{CI}$ & 0.01 & $0.1 \quad 1$ \\
\hline Trudinger $1987^{1}$ & $6 / 127$ & 4.7 & $8 / 162$ & 4.9 & 0.95 & $0.32-2.81$ & & \\
\hline McParland 1988 & not stated & & & & & & & \\
\hline Tyrrell 1990 & $3 / 250$ & 1.2 & $12 / 250$ & 4.8 & 0.29 & $0.10-0.81$ & & \\
\hline Omtzige 1990 & $19 / 825$ & 2.3 & $25 / 815$ & 3.1 & 0.75 & $0.41-1.36$ & & \\
\hline Newnham 1991 & $15 / 275$ & 5.5 & $6 / 270$ & 2.2 & 2.39 & $1.00-5.71$ & & \\
\hline Hofmeyr 1991 & $8 / 438$ & 1.8 & $9 / 459$ & 2.0 & 0.93 & $0.36-2.43$ & & \\
\hline Almström 1992 & $4 / 214$ & 1.9 & $5 / 212$ & 2.4 & 0.79 & $0.21-2.95$ & & \\
\hline Davies 1992 & $9 / 1246$ & 0.7 & $6 / 1229$ & 0.5 & 1.47 & $0.53-4.07$ & & \\
\hline Mason 1993 & $8 / 1015$ & 0.8 & $12 / 1001$ & 1.2 & 0.66 & $0.27-1.59$ & & \\
\hline Johnstone 1993 & $9 / 370$ & 2.4 & $13 / 384$ & 3.4 & 0.71 & $0.31-1.67$ & & \\
\hline Johnstone 1993 & $26 / 1128$ & 2.3 & $29 / 1196$ & 2.4 & 0.95 & $0.56-1.62$ & & \\
\hline
\end{tabular}

${ }^{1}$ Apgar score at 5 minutes less than 6

Table 2.12

Randomised clinical trials

Effectiveness of Doppler ultrasound measurements: Admission to neonatal care unit

\begin{tabular}{|c|c|c|c|c|c|c|c|c|c|c|}
\hline \multirow[t]{2}{*}{ Reference } & \multicolumn{2}{|c|}{ Intervention group } & \multicolumn{2}{|c|}{ Control group } & \multicolumn{2}{|c|}{ Odds ratio } & \multicolumn{3}{|c|}{$\begin{array}{l}\text { Odds ratio and } 95 \% \\
\text { confidence interval }\end{array}$} & \multirow{2}{*}{10} \\
\hline & $\mathrm{n}$ & $\%$ & $\Pi$ & $\%$ & & $95 \% \mathrm{CI}$ & 0.01 & 0.1 & 1 & \\
\hline Trudinger 1987 & $27 / 127$ & 21.3 & $38 / 162$ & 23.5 & 0.88 & $0.51-1.54$ & & & & \\
\hline Mclarland 1988 & not stated & & & & & & & & & \\
\hline Tyrrell 1990 & $18 / 250$ & 7.2 & $19 / 250$ & 7.6 & 0.94 & $0.48-1.84$ & & & & \\
\hline Omtzigt 1990 & $307 / 825$ & 37.2 & $313 / 815$ & 38.4 & 0.95 & $0.78-1.16$ & & & & \\
\hline Newnham 1991 & $103 / 275$ & 37.5 & $106 / 270$ & 39.3 & 0.93 & $0.66-1.31$ & & & & \\
\hline Hofmeyr 1991 & $66 / 438$ & 15.1 & $69 / 459$ & 15.0 & 1.00 & $0.70-1.45$ & & & & \\
\hline Ålmström 1992 & $76 / 214$ & 35.5 & $92 / 212$ & 43.4 & 0.72 & $0.49-1.06$ & & & & \\
\hline Davies 1992 & $44 / 1246$ & 3.5 & $43 /] 229$ & 3.5 & 1.01 & $0.66-1.55$ & & & & \\
\hline Mason 1993 & $29 / 1015$ & 2.9 & $31 / 1001$ & 3.1 & 0.92 & $0.55-1.54$ & & & & \\
\hline Johnstone 1993 & $46 / 370$ & 12.4 & $46 / 384$ & 12.0 & 1.04 & $0.67-1.61$ & & & & \\
\hline Johnstone 1993 & $96 / 1114$ & 8.6 & $101 / 1175$ & 8.6 & 1.00 & $0.75-1.34$ & & & & \\
\hline
\end{tabular}


From the fact that most $95 \%$ confidence intervals of the randomised clinical trials summarised in tables 2.7 to 2.12 include ' 1 ', it can be concluded that the application of Doppler ultrasound measurements in the intervention group does not improve obstetric intervention or perinatal ourcome in comparison with the control group. However, there are several reasons why a valid test does not necessarily lead to a change in outcome (Knotrnerus 1989), as discussed below.

Newnham (1991) noted that local protocols and clinical practice influence the effects of Doppler ultrasound assessments to a large extent. If the local routine testing prevails, regardless of the result of the test under investigation, differences in clinical management, and subsequently in outcome berween intervention and control group, could be less marked than suggested by the study protocol. The additive effectiveness of Doppler ultrasound measurements is in fact tested by some RCTs. Although Doppler ultrasound measurements might have a clinically relevant effect, their additive effect may fail to reach significance.

On the other hand, if clinical management is ineffective, the outcome may not differ between the groups, even if all cases at risk are identified.

As abnormal Doppler flow velocity waveforms have been found to be associated with an increased risk of fetal distress (Divon 1989), a number of clinicians has concluded that when abnormal Doppler results are found, a caesarean section should be performed the same day. This despite the fact that abnormal Doppler ultrasound measurements may be found days or weeks before fetal distress is indicated at CTG examinations (James 1992, Ribbert 1993). The consequences of this attitude are a potential increase of morbidity and mortality associated with prematurity and maternal surgical risks, and an unknown decrease of perinatal morbidity and mortality from acute stress.

There remains doubt wherher the evaluated outcome parameters and the 'gold standards' used, are specific for IUGR. Slowing of fetal growth or postnatal catch-up growth might prove a more sensitive standard for IUGR.

Effects may be magnified if larger study populations are used. On the other hand, if small differences then become statistically significant, their clinical significance is not always clear (see discussion of the RCT of Newnham 1993).

\section{Trudinger 1987}

Objective To determine the effects of early identification of compromised fetuses by Doppler ultrasound measurements on obstetric intervention and gestational age at delivery.

Randomisation By envelopes containing a random number.

Subjects Patients admitted to the antenatal ward for various indications with a GA past 28 weeks were randomised to an intervention group $(n=133)$ and a control group $(n=$ 167). Respectively, 6 and 5 patients in each group delivered at other hospitals and were excluded from the analysis.

Intervention Doppler results had been available for clinical decision making before the start of the trial. In the intervention group, Doppler ultrasound measurements continued 
to be available to the clinicians in charge. Measurements were performed weekly during hospitalisation and the systolic/diastolic ratio (A/B ratio) was calculated. Previously published serial measurements of 15 uneventful pregnancies were used as a reference. In the control group, Doppler ultrasound measurements could no longer be requested. Results The number of patients with an abnormal Doppler result was not stated. Gestational age at delivery did not differ significantly between the groups. The groups had similar rates of elective Caesarean section (CS) and induction of labour. Only the emergency $\mathrm{CSs}$ rate was significantly lower in the intervention group than in the control group. The gestational age at delivery and other parameters of neonatal ourcome did not differ significantly between the groups.

Conclusion of the authors The authors concluded that the availability of Doppler information improved obstetric decision making and neonatal outcome.

Comments The study protocol gives rise to a number of queries. Despite the lack of effectiveness trials, Doppler ultrasound measurements had already been allowed to influence clinical decision making before the start of the study. Therefore, the effect of withdrawing the Doppler information in the control group was investigated rather than its application in the intervention group. S/D ratios obtained from only 15 uneventful pregnancies seern a narrow basis for a reference. A one week gain in gestational ayc at delivery in the intervention group was used to calculate the size of the study. However, the benefit of this gain in gestation in term pregnancies is questionable. The $20 \%$ difference in the size between the two groups indicates a flaw in the randomisation technique.

With regard to the results of the trial, the higher rate of energency Caesarian secrions in the control group compared to the intervention group was not supported by an increase in parameters of neonatal distress in this group.

Doppler ultrasound measurements did nor change neonatal outcome. In conclusion, rhe author's positive conclusion is not corroborated by the results of the trial.

\section{McParland 1988}

Objective Firstly, to reduce perinatal mortality of fetuses with absent end-diastolic frequencies by $20 \%$, and secondly, to demonstrate that a reduction in antenatal stay was possible in patients with normal Doppler waveforms in the umbilical and utero-placental circulation.

Randomisation Patients were stratified on the basis of a normal or an abnormal first Doppler result from the fetal circulation. The randomisation technique was not stated. Subjects Pregnancies complicated by hypertension and/or IUGR. $N=254$ in the intervention group and $\mathrm{n}=255$ in the control group.

Intervention The Doppler results were revealed in the intervention group and concealed in the control group.

Results Perinatal death occurred only in cases with diastolic zero flow and its occurrence rate was significantly lower in the intervention group than in the control group. Whether a proportion of the perinatal deaths was due to fetal abnormalities was not stated. The length of antenatal hospitalisation was significantly lower in the intervention group (10.5 days) than in the control group ( 25.0 days, $p<0.00$ ). 
Conclusion of the authors The authors conclude that the knowledge of the Doppler ultrasound results of both circulations reduced the duration of antenatal hospitalisation and perinatal death.

Comments The description of this trial is far from complete. Whether stratification on the basis of the first Doppler results biased the results or led to ethical problems was not discussed. The randomisation technique was not described. The paper does not state if the clinical management was left to the judgement of the clinicians in charge or if recommendarions for clinical management were made in case of normal or abnormal Doppler results. Such omissions make it difficult to judge the trial's results.

\section{Tyrrel 1990}

Objective Routine versus highly selective use of Doppler ultrasound measurements and biophysical scoring in high risk pregnancies. Main ourcome measures were gestational age at delivery, obstetric intervention rates, and short term neonatal morbidity.

Randomisation By using sequentially numbered envelopes containing a random number. Subjects Pregnancies at high risk of IUGR or still birth, excluding maternal diabetes. 250 patients were allocated to each group.

Intervention From 28 weeks of gestation (or from the time of presentation), the intervention group routinely received Doppler ultrasound measurements and biophysical profile testing, weekly in the first three weeks, and fortnightly thereafter until delivery. Blood velocities were measured in the umbilical artery and utero-placental circulation on both sides. Threshold values for an abnormal test were an A/B ratio > P95 in the umbilical artery, a resistance index $(\mathrm{RI}) \geq 0.58$ in the urero-placental circulation, and a biophysical profile score $\leq 6$, including cardiotocography. Obstetric investigation in the control group could include growth scans, CTGs, and monitoring of fetal movements by fetal kick charts. In this group, Doppler investigations and biophysical assessments could only be obtained on special request and this occurred in 12/250 (4.8\%) of the patients.

Results The A/B ratio in the umbilical artery was raised in 60/250 (24\%), and either A/B ratio or $\mathrm{RI}$ in $95 / 250$ (38\%) of the intervention group patients on one or more occasions. No biophysical profile score was persistently abnormal if Doppler results were normal. Mean gestational age at delivery was significantly lower in the intervention group. Length of antenatal stay and elective delivery rate were not significantly different. However, this information was collected retrospectively, and $15 \%$ of clinical notes could not be traced. With regard to the short term neonatal morbidity, Apgar scores $<7$ at five minures and the occurrence of serious neonatal morbidity were significantly lower in the intervention group. Admission to neonatal care unit, duration of stay, and ventilation time were not significantly different.

Conclusion of the authors The results of the trial have led to the introduction of Doppler ultrasound measurements as a routine test in high risk pregnancies in that centre.

Comments The choice to implement Doppler ultrasound measurements into clinical decision making, was not clearly motivated by the trial's results. Obstetric intervention tates and most paramerers of neonatal outcome were similar in both groups. The positive conclusion was therefore based on a difference in average gestational age at delivery of half a week, a lesser occurrence of serious neonatal morbidity, and better five minute Apgar scores. The difference in GA at delivery was statistically significant but its clinical 
importance is questionable. Serious morbidity involved less than $2 \%$ of the high risk population. Moreover, the $95 \% \mathrm{CI}$ of the odds ratio (0.02-0.98) indicates a marginal benefit.

\section{Omtzigt 1990}

Objective The effectivity of Doppler ultrasound measurements with regard to maternal admission, obstetric management and perinatal outcome in a general outpatient population. Randomisation Performed by maternal date of birth.

Subjects All patients delivered at the study centre, excluding multiple pregnancies with more than two fetuses. Three percent of eligible parients refused to participate. 809 patients ( 825 infants) were randomised to the intervention group, and 789 ( 815 infants) to the control group.

Intervention In the intervention group, Doppler ultrasound measurements could be ordered if indicated. The control group did not have access to Doppler ultrasound measurements and only one patient received Doppler examinations. A management advice was added to the Doppler results ranging from: re-examination after one week if indicated ( $\mathrm{PI}<$ mean $+2 \mathrm{SD})$, re-examination within 1 week (mean $+2 S \mathrm{SD}<\mathrm{PI}<\mathrm{mean}$ $+3 \mathrm{SD}$ ), and hospital admission ( $\mathrm{PI} \geq$ mean $+3 \mathrm{SD}$ ). Termination of pregnancy was indicated if $G A \geq 34$ weeks and absent end-diastolic flow was found.

Results Forty-six percent of patients in the intervention group (368/809) received one or more Doppler examinations and $39(10.6 \%)$ of these had a raised Doppler result. Maternal admission rate and duration, obstetric intervention, and perinatal outcome were not significantly different in the two groups. Overall perinatal death, excluding those due to chromosomal and/or congenital defects, was not significantly different between the study groups. In a subgroup of high risk pregnancies, preventable intrauterine mortality was $1 / 518$ in the intervention group and $4 / 507$ in the control group. The relative risk was 0.24 (95\% CI $=0.03-2.14)$. Neonatal mortality was similar in both groups.

Conclusion of the authors Doppler ultrasound measurements were not shown to be useful in low risk pregnancies. On the basis of the RCT, Doppler ultrasound measurements were introduced at the study centre in the clinical management of high risk pregnancies after 24 weeks of gestation to reduce perinaral dearh. The cost of such a management was estimated at five Doppler examinations per high risk patient.

Comments Because maternal date of birth was used as a randomisation method, the consultant in charge was aware of the randomisation group beforehand. Neonatal assessment was not blinded to the Doppler results. Both are susceptible to bias. The secondary analysis of intrauterine deaths, excluding those due to immaturity or defects, in a subgroup of high risk patients is a narrow basis for the positive assessment of the value of Doppler ultrasound measurements.

\section{Newnham 1991}

Objective To determine the additive value of Doppler ultrasound with emphasis on duration of neonatal stay.

Randomisation After stratification for twin pregnancies, randomisation was performed with sealed, numbered envelopes. 
Subjects A third trimester general high risk population referred for fetal monitoring. 254 patients and 275 feruses were allocated to the intervention group, and 251 patients and 270 fetuses to the control group.

Intervention Only the intervention group was allowed Doppler examinations in the umbilical artery and a maternal utero-placental artery within the placental bed, the frequency being left to the judgement of the ultrasonologist and the clinician in charge. $\mathrm{A} / \mathrm{B}$ ratios were compared to previously published reference ranges.

Results A/B ratios were raised in the umbilical artery in $65 / 254$ (25.6\%) patients in the intervention group. In both groups, the frequency of CTG monitoring was similar and occurred in more than $80 \%$ of the population. The duration of neonatal care admittance was not significantly different between the groups. Neither were other parameters of obstetric care and perinatal outcome.

Conclusion of the authors Introduction of Doppler ultrasound measurements did not influence perinatal outcome or obstetric management.

Comments Notable was that ultrasonic fetal biometry and CTG monitoring were liberally applied in both groups, limiting a possible effect of Doppler ultrasonography to an additive one.

\section{Hofmeyr 1991}

Objective To determine whether the use of Doppler ultrasound measurements could safely reduce the frequency of CTG monitoring. A second objective of this trial, was the reduction of the time taken for testing.

Randomisation By computer-generated algorithm based on the hospital number.

Subjects All women undergoing evaluation of fetal wellbeing.

Intervention The intervention group was primarily evaluated by Doppler ultrasound and the control group was routinely monitored with CTGs.

Results The number of CTGs was lower in the intervention group than in the control group. However, the merhod of fetal monitoring to which the patient was not allocated, was used so liberally that the total number of monitoring occasions was higher in the intervention group and the mean monitoring time was similar in the two groups. No furcher staristical analysis was performed on these data. The number of emergency CSs was significantly lower in the intervention group. Other parameters of obstetric intervention and perinatal outcome were similar in both groups.

Conclusion of the authors The deliberately liberal use of the non-allocated tests in both study groups did not lead to a reduction in fetal monitoring time.

Comments The definition of an abnormal RI was not given, neither was the number of patients with an abnormal Doppler result. Evaluation by the non-allocared method was liberally performed and occurred in $66 \%$ of the monitoring occasions in the intervention group and in $39 \%$ of the monitoring occasions in the CTG group. The number of patients who received both techniques was not stated. The primary objective can, therefore, not be assessed. Doppler examinations were unsuccessful in $26 \%$ of the measurement occasions. This is an unacceptably high failure rate and calls the quality of the measurement technique into question. 


\section{Ålmström 1992}

Objective The objective was to assess whether Doppler flow velocity waveform analysis could replace CTG monitoring in SGA feruses.

Randomisation Performed in blocks with sealed numbered envelopes.

Subjects Women with singleton pregnancies with an ultrasonically estimated fetal weight $<$ mean-2SD at or after 31 weeks of gestation.

Intervention Participants were randomised to fetal monitoring either by means of Doppler ultrasound measurements or by means of CTGs. Patients in the CTG group were not allowed Doppler examinations. All patients received CTG monitoring during labour. Doppler waveforms were evaluated semi quantitatively with blood flow classes iclass I: positive diastolic velocity and $\mathrm{PI} \geq$ mean $+2 \mathrm{SD}$, class II: absent end-diastolic velocity, class III: absent blood flow velocity throughout diastole or reversed diastolic flow). Patients in the normal blood flow class were examined every rwo weeks, every week, or admitred to hospital and examined twice a week, depending on the severity of estimated fetal weight deviation, which was reassessed every two weeks. In blood flow class I, the examination frequency was twice a week, in class II at least twice a week. Blood flow class III was an indication for Caesarean section on the same day. According to the protocol, patients in the intervention group were not allowed CTG monitoring for suspected IUGR alone. All participating patients were given leave from work.

Results Thirty patients in the intervention group (14\%) had antenatal CTGs for other indications. One patient in the CTG group had to be excluded because all the patient notes were lost. By chance, the proportion of smokers was higher in the Doppler than in the CTG group. Fetal monitoring frequency, prenatal admittance, induction of labour, and emergency CS for fetal distress were significantly more frequent in the control (CTG) group than in the intervention group. The significantly increased elective CS rate in the intervention group is due in part to a higher chance occurrence of breech presentation and suspected fetopelvic disproportion in this group. Neonatal outcome was not significantly different.

Conclusion of the authors Doppler velocimetry can be used as sole monitoring test of otherwise uncomplicated SGA pregnancies. This is more cost-effective than CTG monitoring.

Comments Clinical management was also based on estimated fetal weight at ultrasound scanning and on CTG monitoring in $14 \%$ of the intervention group patients. The additive effect of Doppler velocimetry is promising with regard to cost-effectiveness. Whether blood flow class III should automatically lead to an emergency CS on the same day is a matter of debate.

The group of uncomplicated SGA pregnancies could well consist of a high proportion of genetically small fetuses. The perinatal risk in such cases is low. The results of the study provide evidence that Doppler ultrasound measurements could be applied as a more cost-effective monitoring technique in uncomplicated SGA pregnancies.

\section{Davies 1992}

Objective To estimate the effect of Doppler ultrasound measurements in the umbilical and uterine arteries on clinical management during pregnancy and perinatal outcome in a general population, with emphasis on the reduction of prenatal hospitalisation. 
Randomisation Performed in blocks with the sealed envelope method.

Subjects Women with singleton pregnancies visiting the out-patients clinic before the 20 th week of pregnancy. Of the 2600 randomised patients $(79 \%$ of the eligible population), $125(4.8 \%)$ were not included in the final analysis, mainly because they delivered elsewhere. Intervention group $\mathbf{n}=1246$, control group $\mathbf{n}=1229$.

Intervention High risk patients in the intervention group (192) had monthly Doppler examinations, while low risk patients (1054) were examined once at 32 weeks. The control group was nor to receive Doppler ultrasound examinations and only 15 patients $(1.2 \%)$ actually did. In case of abnormal Doppler results, the patient was referred to the antenatal care clinic but clinical management was left to the clinician in charge. A Doppler index $>$ P95 (RI in the urerine artery, PI in the umbilical artery) was regarded as abnormal. Results In the intervention group, 153 parients $(12.3 \%)$ had one or more abnormal results in either measurement location. Of these, $89(58 \%)$ returned to normal and only 7 of the remaining 64 had persistent abnormal findings in the umbilical artery. In 11 cases, the Doppler results were erroneously reported to be normal. Prenatal admission did not differ between the two groups. Neither did perinatal outcome, except for perinatal mortality (table 2.9) which, contrary to expectations, was significantly higher in the intervention group than in the control group.

Conclusion of the author Neonatal outcome was not improved by routine Doppler screening of the general obstetric population. The higher perinatal death in the intervention group in comparison to the control group was attributed to chance.

Comments It was justifiably questioned whether the difference in perinatal death would have been attributed to chance if the results had been reversed (Bowes 1993).

\section{Mason 1993}

Objective To determine whether Doppler ultrasound examinations should become part of rourine prenatal care by comparing its routine use with highly selective use.

Randomisation By sealed opaque numbered envelopes.

Subjects Low risk primigravid women were included at their booking visit. In the intervention group 863/1073 (80\%) participants and in the control group 43/1072 (3.9\%) participants received Doppler examinations. Fifty-three patients in the intervention group, and 67 in the control group, aborted before testing or moved away. These, and five and four twins in each group, respectively, were excluded from the analysis.

Intervention Patients in the intervention group were examined at 28 and at 34 weeks. An $\mathrm{A} / \mathrm{B}$ ratio $>4.5$ was regarded as abnormal. The control group had access to Doppler ultrasound examinations if indicated.

Results The prevalence of abnormal Doppler results in the intervention group was $15 / 863$ $(1.7 \%)$. Obstetric intervention and perinatal outcome did not differ between the groups. Conclusion of the authors Doppler ultrasound measurements did not have a beneficial effect in low risk pregnancies, probably because the incidence of poor outcome is too low. Comments This well designed RCT confirms previous findings that Doppler ultrasound screening in low risk pregnancies does not significantly improve outcome. 
in the intervention group would lead to a more effective obsterric management and at least a similar perinatal outcome, compared to the control group.

Randomisation By sealed opaque envelopes.

Subjects Patients referred for fetal monitoring. Of the total population, 2289 women $(28.5 \%)$ were randomised. The number of fetuses concerned was not so clearly stated. Before the start of the trial, a separate analysis was planned for cases with suspected intrauterine growth retardation and/or hypertension. The subgroup included 370 patients in the intervention group and 384 in the control group. The results of the subgroup are presented first in tables 2.7-2.12, followed by the results of the whole group. Intervention In the intervention group, no restrictions were made on patient management, except that absent end-diastolic flow was considered to indicate either delivery or hospital admission. A RI > mean+2SD was regarded as abnormal. The control group was monitored with accepted techniques and was not allowed Doppler ultrasound examinations. Only three patients in the control group (0.003\%) did.

Results Eight percent of the patients in the intervention group had at least one Doppler result above the normal range (89/1114). The use of CTGs, biophysical profile assessments, and ultrasound scans did not differ significantly between the two groups. Maternal and neonatal admission did nor differ between the study groups. Neither did perinatal outcome. Results were similar in the suspected IUGR/hypertension subgroup.

Conclusion of the authors According to the authors, cardiotocography assesses current fetal wellbeing and Doppler ultrasound measurements assess chronic placental circulatory reserve. As tests they were regarded as complementary. Introduction of Doppler ultrasound measurements into clinical decision making was, therefore, not expected to improve perinatal outcome. The expectation that it might improve obstetric management and reduce monitoring frequency was not confirmed. The authors suspected that clinicians relied mainly on clinical paramerers to determine further management and monitoring frequency rather than on the Doppler results. This effect could leave ourcome parameters unchanged, even if the application of Doppler measurements was effective. Comments The number of fetuses involved in the study was not clearly defined. The number of singletons was stated to be 1095 and 1147 . Respectively, 19 and 28 pregnancies, can be deduced to be 'multiple' without further definition. Respectively, four and six perinatal deaths were associated with congenital and/or chromosomal defects. The number of defects in viable infants was nor stated. The number of normal infants included in the analysis in each group can be approximated to be $1129(1114+19-4)$ and 1197 $(1175+28-6)$, if multiple pregnancies are taken to be twins. In other outcome measures, such as birth weight centile or Apgar score, still other denominators were given, which may have been due to missing data.

Two RCTs were not included in the tables. A randomised trial on the value of Doppler ultrasound measurements as screening test will not be discussed further, as the description of methodology and outcome parameters was insufficient (Schneider 1991). A recent RCT was not included because too few of the outcome parameters were reported (Newnham 1993). The trial is described below, because the protocol and the conclusions of the authors were interesting. 


\section{Newnham 1993}

Objective To compare repeated ultrasonic fetal biometry and Doppler ultrasound examinations with a single ultrasonic examination to test the effect on rate of preterm birth and the duration of neonatal admission.

Randomisation By sealed envelopes containing random numbers in blocks of 20 .

Subjects General population at the outpatient clinic. The intervention group included 1415 patients and the control group 1419 patients. Respectively, 13 and 20 patients were lost to follow-up.

Intervention The intervention group was monitored at $18,24,28,34$, and 38 weeks of gestation. The control group was monitored once at 18 weeks. A/B ratios were calculated from waveforms of an umbilical artery and an arcuate artery.

Results The rate of preterm birth and the duration of neonatal hospitalisation were similar in both groups. Secondary outcome parameters of obstetric management and perinatal outcome did not differ significantly between the two groups, with the exception of SGA. In the intervention group, the proportions of neonates with birth weights for the GA < $\mathrm{P} 10$ and < P3 were found to be greater than in the control group, with a relative risk of $1.35(95 \% \mathrm{CI} 1.09-1.67)$ and 1.65 (95\% CI 1.09-2.49), respectively.

Conclusion of the authors Repeated Doppler ultrasound examinations may increase the proportion of SGA infants.

Comments Although the authors themselves state that the relation between the number of Doppler examinations and the prevalence of SGA infants was not the main objective of their study, the conclusion was nevertheless drawn. Regretfully, the numbers of neonates concerned were not given, so that this finding cannot be reassessed. The difference in mean birth weight between the two groups was a clinically irrelevant 25 grams. Notable is that only $56 \%(821 / 1415)$ of the patients in the intervention group actually received the five planned ultrasound examinations and that, contrary to the study prorocol, $44 \%$ (797/1419) of the patients in the control group was examined more than once. If only the patients monitored according to the protocol would have been used to calculate risks, this would have reduced the value of the denominators. The difference in the occurrence of SGA might then not have been significantly different. Moreover, it is likely that the compliance to the intensive protocol was greater if fetal wellbeing was in doubt, increasing the monitoring frequency in the intervention group. In the control group, the examination frequency would be kept as low as possible in order to comply with the protocol, even in cases with greater fetal risk. In conclusion, such a secondary finding must be treated with caution.

In conclusion, the lack of effectiveness of Doppler ultrasound measurements in low risk populations has been well assessed (Omtzigt 1990, Davies 1992, Mason 1993). At present, it is doubtful wherher Doppler ultrasound technology has sufficiently proven its effectiveness in the identification of pregnancies at risk of IUGR and in the improvement of neonatal outcome in high risk pregnancies. This has not prevented Doppler ultrasound technology from gradually shifting from an object of research into a clinical tool in obstetrics, more through the passing of time than due to a conscious 
and critical evaluation process. From a questionnaire among obstetricians active in the university hospitals in the Netherlands, it is known that at present more than half of these clinics use Doppler ultrasound measurements in clinical decision making (Nienhuis 1994, chapter 7).

In the studies presented above, the objective was to detect cases at increased perinatal risk. Alternatively, Doppler ultrasound could be applied to clear a high risk population of individual cases at low risk, thus increasing the specificity of clinical management. This approach was used in the randomised clinical trial described in the following chaprers of this thesis.

\subsection{Perinatal consequences of intrauterine growth retardation}

\subsubsection{Postnatal (catch-up) growth}

The term catch-up growth was used to indicate the increased growth velocity after a growth restriction (Tanner 1986). When the original growth curve is reached and growth velocity returns to normal, catch-up is thought to be complete. If the genetic growth potential is not fulfilled in utero due to an insufficient intrauterine environment, the neonate will strive to regain its genetic set-point after delivery. There is at present no established definition of catch-up growth after delivery.

In most publications on postnatal growth in SGA infants, an increase in growth rate occurred within the first three or six months after delivery (Davies 1979a, Scott 1982, Villar 1982b, Walther 1982, Brandt 1986, Dunn 1986, Tenovuo 1987, Hitchcock 1989, Fitzhardinge 1989, Albertsson 1993). Hereafter, the postnatal growth velocities of AGA and SGA neonates differed little. Alternatively, some authors have found that postnatal growth velocity was lower in SGA infants (Cruise 1973, Commey 1979, Casey 1990). However, comparison between studies in order to explain these contradictory findings are limited by the differences in the study populations, and in definitions of SGA and catch-up growth.

With regard to the pattern of postnatal catch-up growth, head circumference seems to catch-up in a shorter time span than weight or height (Dunn 1986, Hitchcock 1989). Weight is increased more than height, so that, although most infants remain smaller than their AGA controls at the end of the first year, their proportions are similar (Fergusson 1980). In general, growth velocity was higher and the final anthropometric values were closer to the mean in SGA infants who were disproportioned at birth than in SGA infants who were proportioned (Davies 1979b, Villar 1982b, Tenovuo 1987). The proportion of SGA infants which did not catch-up, but remained small, ranges from 10 to $30 \%$ in the different studies (Davies 1980, Ounsted 1982a, Fitzhardinge 1989 , Albertsson 1993). If parental size was reported, the parents were often found to be small. This suggests that these infants could be genetically small (Ounsted 1982a). 
One might conclude that the occurrence of postnatal catch-up growth could be used to retrospectively differentiate the growth retarded infant from the generically small infant (Davies 1981). As a true gold standard for IUGR, defined as the 'best measure of the truth against which the accuracy of a diagnostic test can be tested' (McParland 1988), is lacking, postnatal catch-up growth seems a good candidate.

A good correlation with anthropometric values at 12 months, is found at two months in AGA infants and at four months in SGA infants (Moar 1982). This suggests that all infants 'tune' into their genetic programming in the first months after delivery. This phenomenon in early infancy was reviewed by Tanner (1986).

A method to standardise anthropometric measurements for age is by calculating the standard deviation score (SDS). SDSs, also called Z-scores, express the number of standard deviations that a measurement differs from the mean at a certain age. SDSs are discussed in more detail in section 3.3.4. An upward shift of more than one SDS in weight, height, and head circumference in SGA infants has been described in the first year after delivery by Davies (1980) and in the first six months after delivery by Scott (1982). An upward shift of at least one SDS in the first six months after delivery could, therefore, be taken as criterion for the occurrence of a significant catch-up growth and therefore as retrospective gold standard for intrauterine growth retardation. The SDS shift used to define catch-up growth must be large enough to exclude physiological 'tuning' or regression towards the mean and small enough to identify less severe growth retardation. Establishing such a threshold value requires more specific study. At present the choice to define catch-up growth by an upward shift of one SDS in weight in the first six months seems a defendable one.

\subsubsection{Mortality and morbidity}

Few publications quantify perinatal morbidity and mortality by their relation to birth weight and gestational age. They generally showed a decreasing mortality with increased weight and gestational age at birth (Goldstein 1981, Koops 1982) although this relation was not linear (Myers 1989). In a recent study, growth retardation was associated with a higher fetal mortality rate, but a lower neonatal mortality rate, compared to their appropriate for gestational age counterparts (Herman 1993). This would suggest that growth retarded infants are more mature than AGA neonates of the same weight. Perinatal mortality related to being small has decreased in the last decades due to improved antenatal and neonatal care (Tenovuo 1988).

Reduced nutrient and oxygen supply, has several consequences. Adaptive mechanisms to hypoxia include redistribution of fetal blood in favour of the brain and the myocard, at the expense of kidneys, intestines, and extremities (Peeters 1979). Decreased renal perfusion is clinically detected by oligohydramnios. Chronic hypoxia induces a number of compensatory adaptations, such as polycythemia, reduced body movements, and cessation of tissue accretion. When these compensatory mechanisms become inadequate, metabolic acidosis will develop as a consequence of anaerobic metabolism. 
Efforts to reduce perinatal morbidity and mortality have led to increased Caesarean section rates (Bolte 1987), which again leads to a rise in the incidence of prematurity. This has resulted in an increasing proportion of preterm SGA infants with a lower mean birth weight. The consequence of this development for maternal morbidity, maternal mortality, and costs remains unclear.

The trend of decreasing perinatal mortality has been shown to be associated with an increased rate of neurological morbidity especially in very preterm infants (Hagberg 1989). If 'utero-placental insufficiency' is severe or protracted, fetal adaptability will be exceeded and asphyxia will compromise the central nervous system. The effects of detrimental influences are dependent on their timing relative to the stages of normal brain development (Herschkowitz 1988). Each stage has a more or less fixed chronological timing during and after gestation (Dobbing 1971), but timing differs between brain regions. The growth spurt of the cerebrum, theoretically the period of highest vulnerability, is from mid gestation to the age of 18 months or 2 years (Dobbing 1973). The growth spurt in the cerebellum begins later and finishes earlier. The peak velocity in weight gain of the fetal brain is between 32 and 37 weeks of gestation (Brandt 1981). In the third trimester of pregnancy, 'placental insufficiency' could affect synaptic connections or growth of the cerebellum. In neonates suspected of IUGR, cerebral weight is reduced most in absolute terms, but the cerebellum most in terms of percentage (Sarma 1974). This may be responsible for the motor coordination of the 'clumsy child'. However, the development of muscle innervation is also influenced by spontaneous activity of the fetus (Hersch kowitz 1988). This could theoretically be of influence if fetal activity is decreased, for instance due to a restricted intrauterine volume in oligohydramnios.

It is far from clear what the functional consequences of the above factors are. Hard figures on neurological risks of IUGR alone are not available, as IUGR is a heterogeneous problem and is often associated with other adverse conditions. The aetiological factors of growth retardation (smoking, hypertension, vascular disease) and the consequences of IUGR (prematurity, hypoxia, hypoglycaemia) also influence the fetal brain directly and subsequent neurological development. Hypoxia especially is a major determinant of neurological outcome in IUGR infants (Commey 1979, Schauseil-Zipf 1989). A recent review on full-term SGA infants concludes that there are no major neurological consequences in the majority of such infants, but that there is an increased risk of minor neurological dysfunction around school age, which is further increased if problems occur in the neonatal period (van Kranen-Mastenbroek 1993).

There is some evidence that infants from stressed pregnancies are neurologically and pulmonary more mature than unstressed infants (Amiel-Tison 1991). Advanced maturity is possibly an adaptive response mediated by stress hormones. Moreover, structural damage of the brain may be compensated for or may be repaired as the greater part of the brain growth spurt is postnatal (Dobbing 1973). Dysfunctions may manifest 
themselves in the neonatal period and then disappear for months or years (Prechtl 1977, Rubin 1980, Brandt 1981, Nelson 1982, Michaelis 1993). Ultimately neurological development depends on the adaptive capacity of the brain and is mediated by environmental stimulation (Rubin 1980, Winick 1981). This may explain the limited predictive value of neonatal neurological development tests on long term neurological development (Korner 1989). Moreover, long term problems generally arise at the level of the higher cortical funcrions. These cannot be fully tested in the neonatal period.

However, neonates who are neurologically 'suspect' after delivery or who are repeatedly found to be neurologically 'suspect' or 'abnormal' in the first year, have a higher risk of developing problems at school age (Prechtl 1977, Nelson 1979, Rubin 1980, AmielTison 1986, Hadders-Algra 1987). Alternatively, infants who are normal at birth have a high probability of remaining normal in childhood.

With regard to non-neurological longterm morbidity, there is some evidence that SGA is associated with an increased risk of hypertension, non-insulin dependent diabetes mellitus, and atherosclerosis associated morbidity and mortality later in life (Gennser 1988, Barker 1990, Barker 1992). Further prospective evaluation in large study populations is needed to elucidate this relation.

\subsection{Therapeutic possibilities}

Therapeutic possibilities in IUGR are limited. Maternal medical conditions such as hypertension may be corrected and habits adverse to fetal growth such as smoking limited. In the Netherlands, bed rest in hospital is often advised if the growth deficit is three weeks or more (Nienhuis 1994, chapter 7). Bed rest is thought to improve fetal growth in suspected IUGR, although studies have not corroborated this (Laurin 1987b). Rourine hospitalisation in twin pregnancies has not been shown to have a positive effect on birth weight or neonatal outcome (Crowther 1989). However, uterine blood flow has been shown to be higher in horizontal position than in standing position (Clark 1989), which theoretically has a positive effect on placental haemodynamics. Effects of maternal hyperoxygenation and haemodilution are still under investigation (Visser 1991). Prevention of IUGR by low-dose aspirin has nor fulfilled expectations (CLASP 1994).

\subsection{Study objective}

In conclusion, the growth retarded fetus or neonate is more prone to perinatal and longterm morbidity and mortality than its appropriate for gestational age counterpart. The reviewed tests identify SGA with various success. But true growth retardation 
cannor yet be adequately defined or identified. This could lead to the development of wrong parameters or wrong treatments and impede the proof of possible effective diagnostic tests or therapies. Moreover, such a state of affairs causes unnecessary parental anxiety and burdens patients with unnecessary clinical fetal monitoring.

Although it may not be possible to identify IUGR reliably, it is of financial importance to exclude IUGR, either qualitatively or quantitatively. A less costly, but no less effective clinical policy may then be obtained.

Reduced umbilical blood flow velocities, as measured by Doppler ultrasound, are thought to be associated with intrauterine growth retardation and fetal distress. However, the validity of Doppler ultrasound measurements in the detection of small feruses is disappoincing, even in high-risk populations.

On the other hand, Doppler ultrasound measurements may be used to exclude IUGR. Some authors have stated that if suspected IUGR is associated with umbilical Doppler ultrasound measurements in the normal range, the risk of perinatal distress is low (Reuwer 1987, Haddad 1988, Burke 1990). It can be hypothesised that costly in-hospital fetal monitoring can be refrained from in such cases, without this being detrimental to perinatal outcome. This dual hypothesis was investigated in the randomised clinical trial described in chapters 3 to 6 of this thesis. 


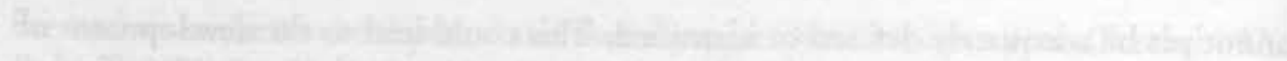

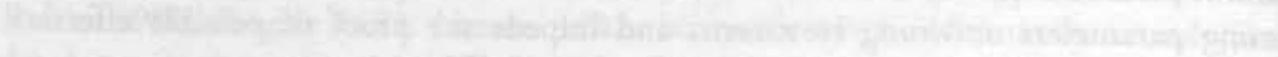
-

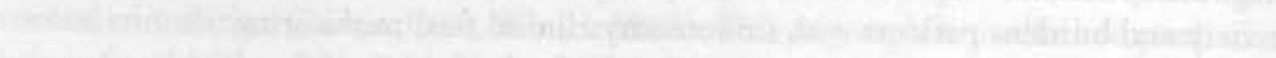

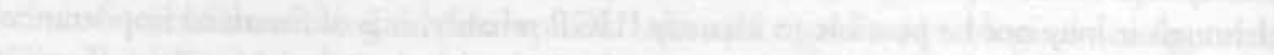

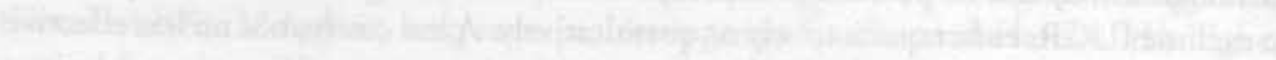

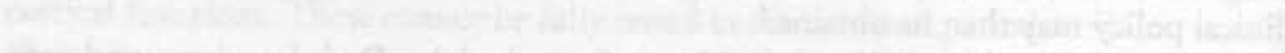

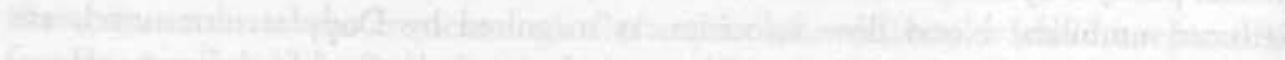

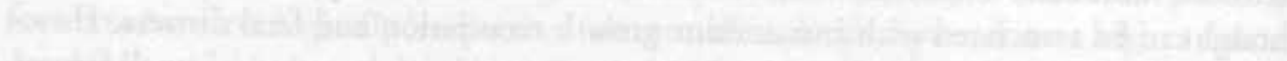

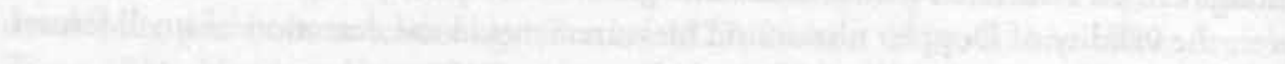

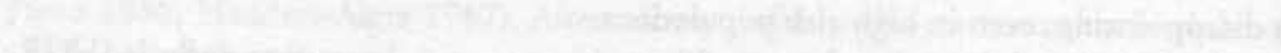

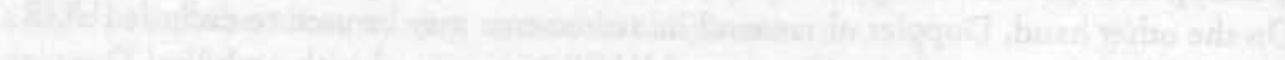

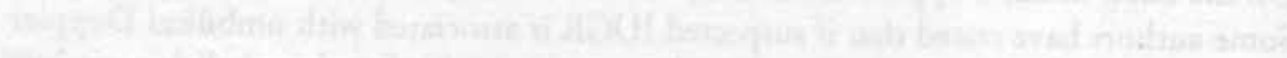

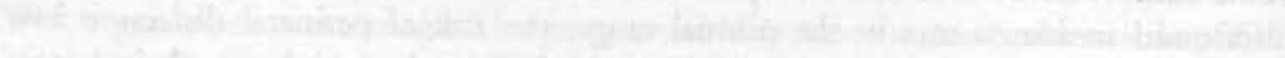

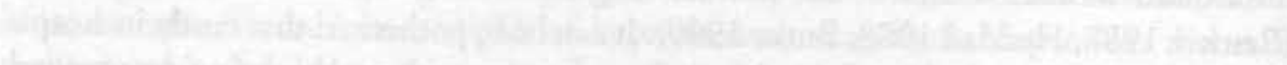

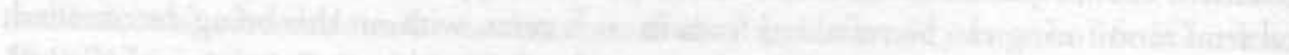

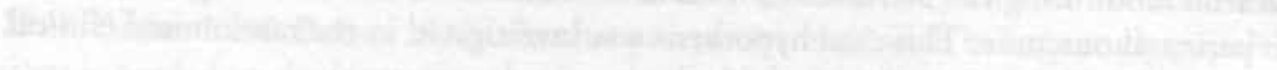

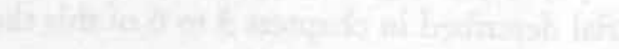




\section{Chapter 3}

\section{Randomised Clinical Trial: Study Protocol}

The following chapters describe the randomised clinical trial on the costs and effects of Doppler ultrasound measurements in suspected intrauterine growth retardation. Umbilical artery Doppler ultrasound measurements are used as 'reassurance test' in clinically suspected intrauterine growth retardation. If the Doppler ultrasound measurements are within the normal range, the pregnancy is not regarded as at high risk and unnecessary in-hospital fetal monitoring is prevented.

The dual hypothesis of this randomised clinical trial is that the application of Doppler ultrasound measurements as a reassurance test in suspected IUGR:

1. leads to a less costly policy by decreasing unnecessary prenatal hospitalisation

2. has no detrimental effect on perinatal outcome

in the intervention group, in comparison to the control group.

The study protocol of the RCT is presented in this chapter. The protocol describes the study population and the Doppler ultrasound measurement technique with the associated clinical policy. The method of cost assessment is described. The effects of the RCT are evaluated by follow-up of postnatal neurological development and growth.

\subsection{Study population}

\subsubsection{Research institute}

The randomised clinical trial was conducted at the Department of Obstetrics and Gynaecology, in collaboration with the Departments of Child Neurology and Paediatric Endocrinology of the University Hospital Maastricht, and the Departments of Health Economics and Methodology and Statistics of the University of Limburg, Maastricht, The Netherlands.

The University Hospital Maastricht (AZM) acts as a general hospital for the city of Maastricht and its direct vicinity, and as regional tertiary referral centre. Participants were derived from a population of pregnant women routinely visiting the obstetric outpatient clinic at the AZM. Some characteristics of this population are shown in table 3.1.

In the Netherlands, midwives are in charge of prenatal care in approximately $50 \%$ of pregnancies and deliveries. If maternal or fetal complications such as intrauterine growth 
48 | Chapter 3

Table 3.1

Outcome of 647 singleton pregnancies at the AZM outpatient clinic in $1989^{1}$

\begin{tabular}{lrr}
\hline Outcome & $\mathrm{n}$ & $(\%)$ \\
\hline induction of labour & 88 & $(13.6)$ \\
elective Caesarean section & 19 & $(2.9)$ \\
emergency Caesarean section & 41 & $(6.3)$ \\
& & \\
premature birth & 75 & $(11.6)$ \\
birth weight $\leq 2500$ grams & 74 & $(11.4)$ \\
birth centile < P10 & 109 & $(16.8)$ \\
breech delivery & 72 & $(11.1)$ \\
intrauterine fetal death & 12 & $(1.9)$ \\
chromosomal and/or congenital defects & 22 & $(3.4)$ \\
\end{tabular}

1 excluding multiple pregnancies, referrals during delivery, and intrauterine transferrals. The selected singleton pregnancies make up $71.1 \%$ of the total number of deliveries at the AZM in 1989 ( 910 deliveries, 932 neonates).

retardation are suspected, the patient is referred to the obstetric outpatient clinic for further evaluation.

The frequency of routine antenatal visits is as follows: every four weeks until the 24th week of gestation, every three weeks from 24 to 32 weeks, fortnightly until the 36th week, and at least weekly from 36 weeks onwards. The frequency of visits is increased if the medical condition of mother or fetus warrants it.

\subsubsection{Inclusion}

The study protocol was approved by the Hospital Ethics Committee in 1989. Inclusion started on September 1st, 1989.

The sole inclusion criterion was:

- a clinically suspected intrauterine growth retardation of two or more weeks diagnosed by fundal height measurements at the outpatient clinic

Excluded were the following cases:

- multiple pregnancies

- uncertain gestational age

- non-Caucasian origin

- requiring immediate hospitalisation or obstetric intervention because of the severity of the maternal or fetal condition

The first appointment with the investigator was made directly after the clinical suspicion of intrauterine growth retardation arose at the outpatient clinic. The study protocol was 
explained to the patient and informed consent was obtained. General information on prognostic factors for fetal growth was collected (presented in section 4.1).

The required number of participants was reached on May 1st 1992. The follow-up was completed in March 1993.

\subsubsection{Stratification and randomisation}

Participants were stratified and then randomised over an intervention and a control group. Stratification was performed to obtain an equal distribution of two confounding factors which could be reliably ascertained: gestational age at randomisation and smoking.

Gestational age (GA) is routinely assessed at the AZM in early pregnancy by ultrasonic fetal biometry. Before a gestational age of 14 weeks the crown-rump length (CRL) was used to assess GA, and thereafter until 20 weeks GA, the biparietal diameter (BPD). Gestational age at inclusion was scored as GA $\leq 32$ weeks or GA $>32$ weeks. Participants were scored as smoker or non-smoker, regardless of the number of cigarettes smoked. A non-smoker was defined as someone who had never smoked or had quit smoking before or at the time of the positive pregnancy test. The stratification criteria led to four randomisation lists (table 3.2), which were prepared in advance from a published table of random numbers (Pocock 1983). A block size of 10 was used.

A randomisation number was requested over the telephone from an independent person not involved in patient management. After stating the gestational age and smoking habit, the next number of one of the four randomisation lists was read (table 3.2). An even number allocated the participant to the intervention group and participants with uneven numbers were allocated to the control group. The use of four lists made prediction of the randomisation group by the referring clinician or the investigator highly unlikely.

Table 3.2

Randomisation lists

List no. Stratification criteria

1. $\mathrm{GA} \leq 32$ weeks / non-smokers
2. $\mathrm{GA} \leq 32$ weeks / smokers
3. $\mathrm{GA}>32$ weeks / non-smokers
4. $\mathrm{GA}>32$ weeks / smokers

\subsection{Doppler ultrasound measurements in the umbilical artery}

The method of the Doppler ultrasound measurements used in this RCT are described in section 3.2.5. In the preceding sections, some background literature is presented on the Doppler ultrasound technique, the pathophysiology underlying the Doppler flow 
velocity waveforms, the accuracy and reproducibility, and the safety of Doppler ultrasound measurements in obstetrics.

\subsubsection{Technical background}

Since the introduction of Doppler ultrasound measurements in obstetrics (FitzGerald 1977), it is possible to measure fetal blood flow velocities non-invasively. Blood flow velocities are assessed by calculating the difference between the frequency of the ultrasound waves emitted by the transducer and the frequency of the waves reflected to the transducer by moving red blood cells. This change in frequencies is called the Doppler shift and is expressed by the following formula:

$$
\begin{array}{ll}
f_{d}=\frac{2 \cdot V \cdot f_{e} \cdot \cos \alpha}{C} \\
f_{d}=\text { Doppler shift } \\
V=\text { velocity of the red blood cells } \\
f_{e}=\text { emission frequency of the ultrasound bundle } \\
\cos \alpha=\text { cosine of the angle between the ultrasound bundle and the direction } \\
C \quad=\text { of blood flow } \\
\text { velocity of ultrasound in human tissue (approximately } 1540 \mathrm{~m} / \mathrm{s} \text { ) }
\end{array}
$$

The technical and physical aspects of Doppler ultrasound are described extensively in a paper by Ruissen (Ruissen 1987). Simplified, the Doppler shift is converted to a velocity waveform versus time (figure 3.1). The typical shape of the waveform reflects the changes in blood velocity and is a result of the forward motion by the contraction of the heart, modified by characteristics of the vessel walls and placental resistance. Two kinds of Doppler techniques are in use: Continuous Wave (CW) and Pulsed Doppler (PD). CW Doppler continuously emits ultrasound signals with one crystal and receives the backscattered ultrasound signals with another. In Pulsed Doppler, ultrasound waves are emitted in bursts and the time in between is used to receive information with the same crystal. Because of the time difference between emission and receiving, the distance travelled by the ultrasound is known and the measurement window can be located within a particular position of interest. A beam diamerer and a sample length of $4 \mathrm{~mm}$ give a sample volume of about $50 \mathrm{~mm}^{3}$, which is satisfactory for most measurements performed in obstetrics (Ruissen 1987). The received Doppler ultrasound information is converted into an audible signal and the amplitudes, which reflect the intensity of each frequency (which in turn reflects each velocity), are displayed against time for further processing. Low-frequency, high-amplitude signals, such as those generated by slowly moving vessel walls, are removed by a high-pass filter. With the emission frequencies used in obstetrics, a filter setting of $100 \mathrm{~Hz}$ or less is generally acceptable. If the filter is too high, low blood velocities during the diastolic phase of the cardiac cycle may fall 
below the filter cutoff frequency and give the impression that there is no diastolic flow (diastolic zero flow).

Part of the emitted ultrasound is not received back because of absorption and scattering by the tissues. This so called attenuation is dependant on the emitted frequency and the distance travelled by the ultrasound. The intensity of the received Doppler information is a trade-off between backscattering, which is maximal if the emission frequency is high, and attenuation, which is minimal if the emitted frequency is low. Considering these two contradictory demands and the depth at which the vessels of interest are generally found in obstetrics, an emission frequency of $2-4 \mathrm{MHz}$ is optimal.

Doppler ultrasound technology was primarily used to calculate volume blood flow, but this proved to be inaccurate due to difficulties in measuring the angle $(\alpha)$ between the ultrasound bundle and the direction of flow. The development of indices to describe Doppler waveforms, made Doppler ultrasound measurements relatively independent of the angle of measurement. Concurrently used indices are: the pulsatility index (PI) (Gosling 1975), the A/B ratio (Stuart 1980), also called the S/D ratio, and the resistance index (RI) (Pourcelot 1982). The PI is defined as the difference between the maximal systolic $(S)$ and the minimal diastolic value $(\mathrm{mD})$, divided by the time averaged mean $(\mathrm{M})$, the $\mathrm{A} / \mathrm{B}$ ratio is defined as the maximal systolic value divided by the end-diastolic value $(\mathrm{eD})$, and the $\mathrm{RI}$ as the maximal systolic minus the end-diastolic value divided by the maximal systolic value of the signal (figure 3.1).

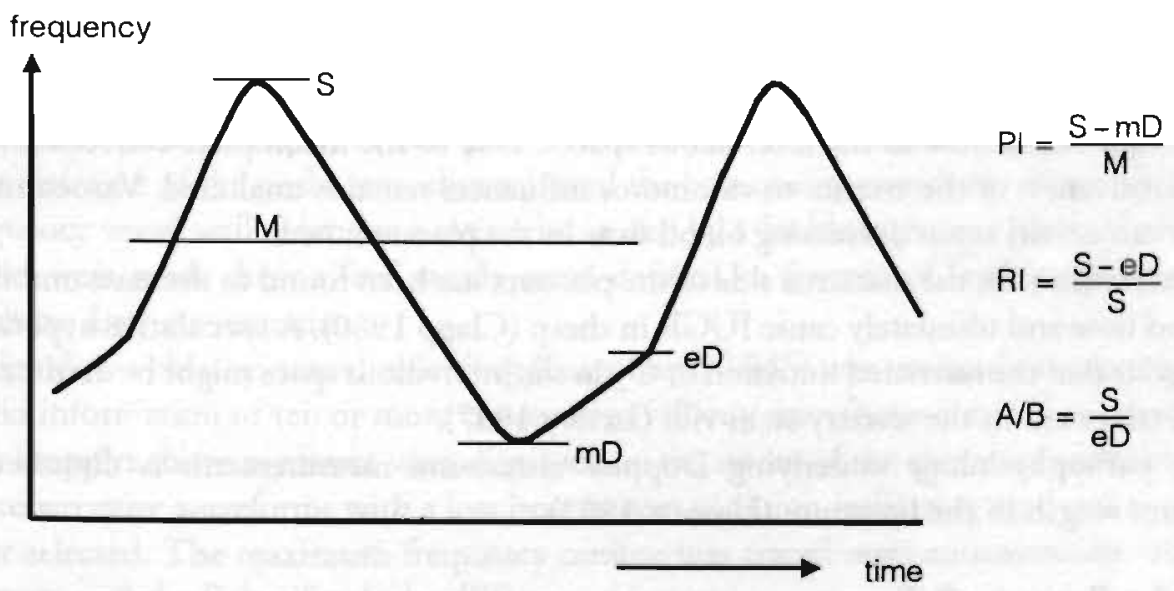

Figure 3.1

Doppler flow velocity waveforms and indices

$\mathrm{PI}=$ pulsatility index, $\mathrm{RI}=$ resistance index $\mathrm{A} / \mathrm{B}=\mathrm{A} / \mathrm{B}$ ratio or systolic to diastolic ratio;

$S=$ maximal systolic value, $\mathrm{mD}=$ minimal diastolic value, $M=$ time averaged mean, $\mathrm{eD}=\mathrm{end}$-diastolic value 
There is zero flow in the umbilical artery if there is no (detectable) flow during the diastolic or relaxation phase of the heart cycle. In the so called reversed flow, blood in the umbilical artery flows in the direction of the heart during the diastolic phase.

In umbilical artery studies, to which the scope of this thesis is limited, use of the PI is preferred as it remains meaningful in case of zero flow or reversed flow.

\subsubsection{Pathophysiology}

The pathophysiology, underlying changes found in Doppler ultrasound measurements, has not been fully elucidated. The aspects of pathophysiology discussed in the literature are summarised below.

With regard to the fetal side of the placenta, the number of small muscular arteries in tertiary stem villi was found to be reduced in placentas associated with abnormal umbilical artery Doppler results, in comparison to that of uneventful pregnancies and high risk pregnancies with normal Doppler results (Giles 1985, McCowen 1987, Bracero 1989). The reduced density of small muscular arteries was thought to increase vascular resistance, thereby primarily reducing diastolic blood flow, which in turn leads to increased Doppler waveform index values. The resulting decreased placental perfusion, and therefore nutrient and $\mathrm{O}_{2}$ availability, could explain the high proportion of SGA neonates in the abnormal Doppler groups.

In the process of physiological placental maturation on the maternal side of the placenta, trophoblast invades the spiral arteries. These thick-walled, muscular arteries are converted into thin-walled, distended utero-placental vessels. Utero-placental vessels are functionally low pressure, high conductance vessels. In pregnancies associated with hypertension and/or IUGR, conversion does not extend into the myometrial part of the spiral arteries (Brosens 1977, Robertson 1981, Khong 1986). This subsequently restricts maternal blood flow to the intervillous spaces. Due to the incomplete conversion, the responsiveness of the arteries to vasomotor influences remains unaltered. Vasoconstriction can occur, again decreasing blood flow in the placental bed.

Interference with the maternal side of the placenta has been found to decrease umbilical blood flow and ultimately cause IUGR in sheep (Clapp 1980). A speculative hypothesis suggests that the restricted nutrition or $\mathrm{O}_{2}$ in the intervillous space might be detrimental to fetal vessels in the tertiary stem villi (Lavery 1987).

The pathophysiology underlying Doppler ultrasound measurements is discussed at greater length in the literature (Hasaart 1993).

\subsubsection{Reproducibility}

Inter-observer and intra-observer variation of the measurements in the umbilical artery were previously examined and found to be acceptable (Nienhuis 1988). Correction for fetal heart rate within the physiological ranges of 120-160 beats per minute was not found to be necessary (Gudmundsson 1988, Legarth 1989). The influence of measurement site on the PI in the umbilical artery is low (Ruissen 1990). 


\subsubsection{Safety}

At present, no detrimental effects of diagnostic ultrasound have been established. Potential effects of Doppler ultrasound on birth weight have been discussed in the previous chapter (Newnham 1993, section 2.5.3). Long term effects on neurological development in humans have been assessed by follow-up of 2428 children aged 8-9 years who had been exposed to routine ultrasound screening during fetal life (Salvesen 1992, Salvesen 1993). Participants in the routine ultrasound group received an average of 2.3 scans. A higher frequency of non-right handedness in the routine ultrasound group (odds ratio $=1.32,95 \%$ CI 1.02-1.71) was the only significant difference between the groups. However, data on handedness were complete in only $69 \%$ of eligible children. If the analysis was limited to left handedness, the difference was not significant (odds ratio = $1.34,95 \%$ CI $0.90-2.00$ ). There was no evidence of a detrimental influence of ultrasound on longterm neurological development and school performance.

\subsubsection{Measurement technique}

The participant was placed in a semi recumbent position and tilted laterally by placing a small cushion under one hip. After ten minutes of rest, the Doppler ultrasound measurements were performed. The total examination time was 15 to 20 minutes. Blood flow velocities in an umbilical artery were measured by pulsed Doppler. The umbilical cord was identified in real time using a $3 \mathrm{MHz}$ mechanical sector scanner (Pie Medical 1120 scanner and Pie Medical 900 transducer) with a $2 \mathrm{MHz}$ pulsed Doppler (PD) device (Pedof) attached in a fixed angle $\left(60^{\circ}\right)$. Aided by the real-time image, the angle between the ultrasound bundle and the direction of flow was minimalised. The sample volume (approximately $5 \times 5 \mathrm{~mm}$ ) was adjusted so that it enclosed the whole vessel. After switching over to the Doppler mode, the quality of the signal was evaluated by ear and by online display of the signal on the monitor of a spectrum analyser (Doptek). The direction of the probe was adjusted until the best quality signal was obtained. Low frequency vessel wall movements were eliminated by a $50 \mathrm{~Hz}$ high pass filter. Measurements were made during fetal steady state, defined as absence of fetal breathing and excessive fetal movements.

A digital two channel sound recording (Sony Super VHS) was made of the quadrature audio information of ten or more consecutive velocity waveforms for off-line analysis. The Doppler shift waveforms were displayed on the screen of the spectrum analyser and six consecutive waveforms with a low beat to beat variation indicating fetal quiescence, were selected. The maximum frequency outline was traced semi-automatically with a lightpen and the Pulsatility Index (PI) was computed.

The PI result was calculated from the average of six waveforms to provide a representative value (Spencer 1989, Kurmanavichius 1989). The reference values of the umbilical artery PI used, were previously established in the AZM outpatient population by van Vugt in a longitudinal study of uneventful singleton pregnancies (van Vugt 1988a, figure 3.2 ). 


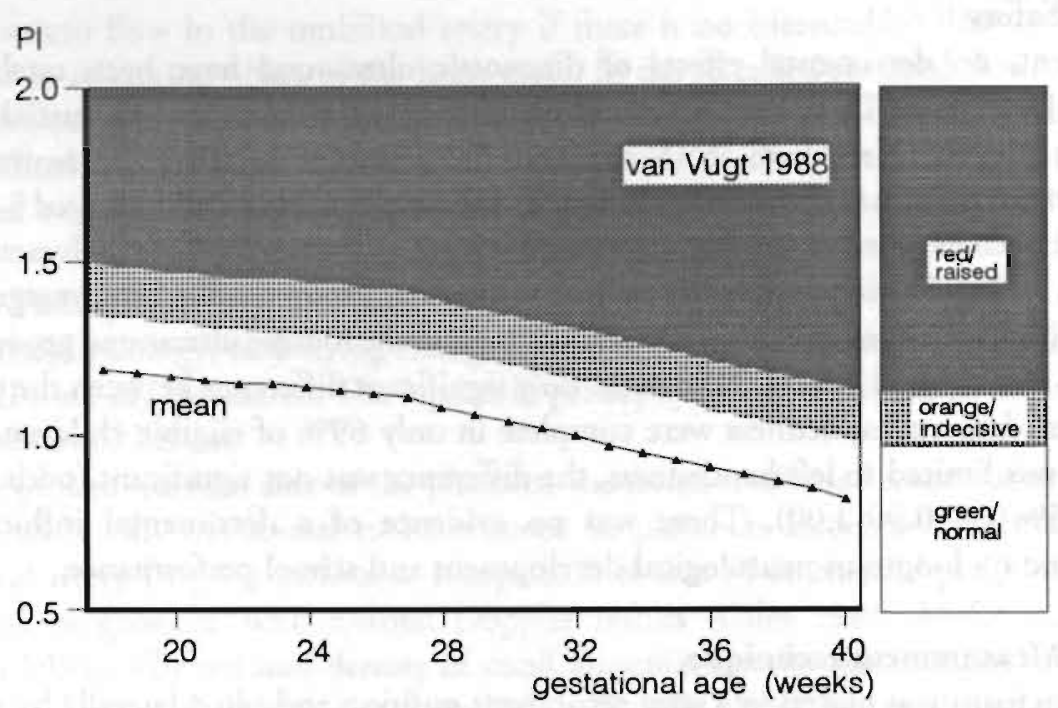

Figure 3.2

Colour coded Pulsatility Index reference values (van Vugt 1988a)

A PI value was defined within the normal range when below the mean plus one standard deviation (PI $<M+1 S D$ ) for the gestational age. A PI value was defined as raised when above the mean plus two SD ( $\mathrm{PI}>\mathrm{M}+2 \mathrm{SD}$ ) for the gestational age. This category also included results whereby diastolic flow is absent, the so called zero flow or absent end diastolic flow, or even reversed during the diastolic phase. A value on and between one and two standard deviations above the mean $(M+1 S D \leq P I \leq M+2 S D)$ for the gestational age was defined as of indecisive clinical value. The final result was colour coded. A normal Doppler result was indicated by the colour green, a raised result by the colour red, and the colour orange indicated an indecisive result (figure 3.2).

The reference PI values used in the trial described in this thesis correspond well with other published values especially from the 24 th week of gestation onwards (table 3.3). This is illustrated by plotting the means of these published values over the reference values used (figure 3.3). The PI values in the umbilical artery in normal pregnancies can be seen to decrease with the gestational age.

\subsubsection{Doppler ultrasound measurements in intervention and control group}

All participants were subjected to weekly Doppler ultrasound examinations by the investigator until delivery. Doppler ultrasound results were only revealed to the doctor or midwife in attendance if the participant had been randomised to the intervention group. The Doppler examinations were performed directly before the visit to the outpatient clinic and the result was conveyed by the respective coloured label in the patients notes. 
Table 3.3

Umbilical artery Pulsatility Index reference values in the literature

\begin{tabular}{lrllllll}
\hline Reference & N & L/C-S & PD/CW & $\begin{array}{l}\text { freq } \\
(\mathrm{MHz})\end{array}$ & $\begin{array}{l}\text { filter } \\
(\mathrm{Hz})\end{array}$ & $\begin{array}{l}\text { GA } \\
\text { (weeks })\end{array}$ & $\begin{array}{l}\text { PI } \\
(\mathrm{GA}=26-40)\end{array}$ \\
\hline Reuwer 1986 & 75 & L & CW & 4 & 200 & $24-40$ & $1.15-0.70$ \\
Wladimiroff 1987 & 149 & C-S & PD & $3.5 / 3$ & - & $26-39$ & $1.19-0.80$ \\
van Vugt 1988a & 96 & L & PD & 2 & 100 & $15-40$ & $1.11-0.83$ \\
Pearce 1988 & 34 & L & PD & - & 150 & $16-40$ & $1.00-0.72$ \\
Gudmundsson 1988 & 125 & C-S & PD & 3.5 & 100 & $20-41$ & $1.29-0.83$ \\
Årström 1989 & 22 & L & PD & 3.5 & 100 & $24-42$ & $1.40-0.87$ \\
Arduini 1990 & 1556 & C-S & PD & $3.5 / 5$ & 100 & $20-42$ & $1.20-0.91$ \\
Fogarty 1990 & 85 & L & CW & - & 200 & $16-40$ & $1.24-0.87$ \\
\hline
\end{tabular}

$\mathrm{N}=$ number of patients; $\mathrm{L}=$ longitudinal, $\mathrm{C}-\mathrm{S}=$ cross-sectional; $\mathrm{PD}=$ pulsed Doppler, $\mathrm{CW}=$ continuous wave Doppler; freq = ultrasound frequency emitted by transducer; filter $=$ high pass filter; $-=$ not stated; The PI values obtained by Pulsed Doppler and continuous wave Doppler did not differ significantly (van Vugt 1988b).

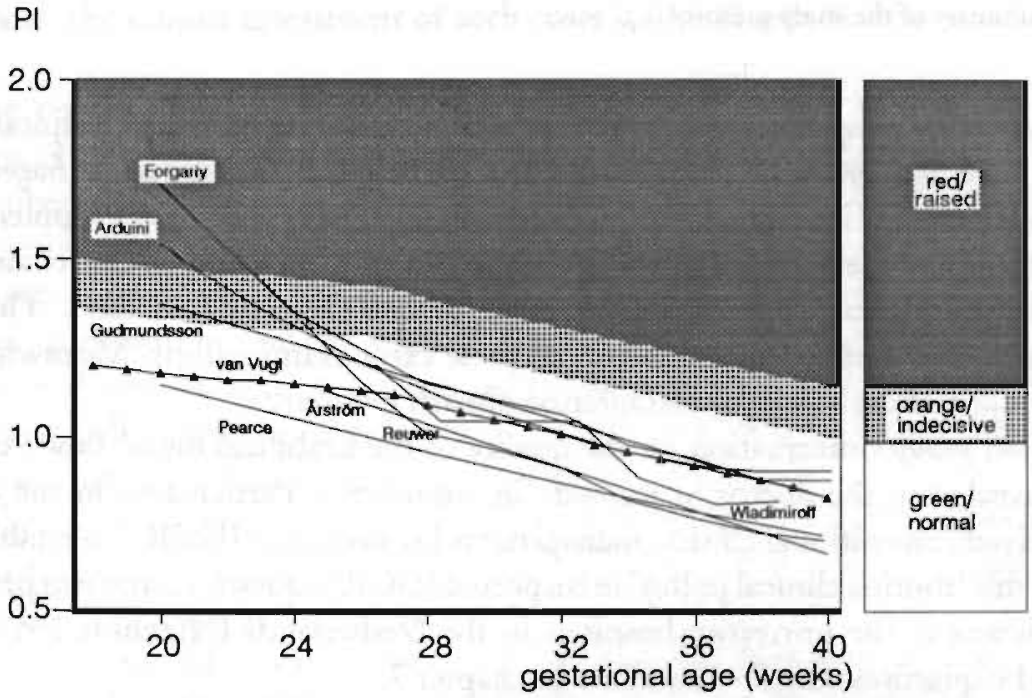

Figure 3.3

Colour coded PI reference values (van Vugt 1988a) and mean PI values in the literature (table 3.3)

Weekly Doppler ultrasound measurements were also performed in the control group, firstly as a placebo intervention and secondly to enable the full results to be analysed at the completion of the study. The results in the control group were concealed from the clinician in charge. A summary of the study protocol is given in figure 3.4. 
Inclusion Randomisation Test Intervention

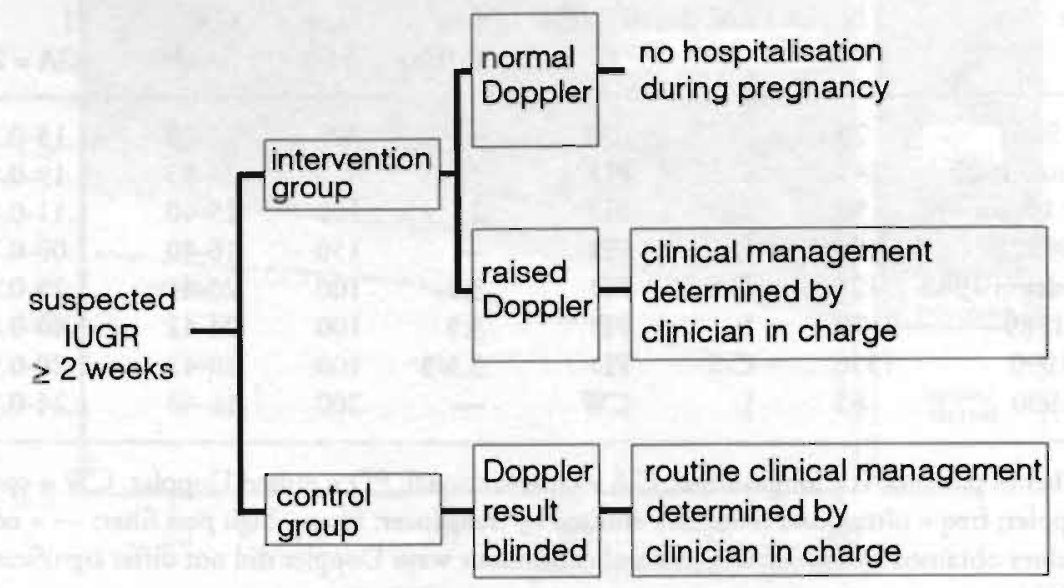

Figure 3.4

Summary of the study protocol

In the intervention group, the Doppler results were linked to a predefined hospitalisation policy. In case of a green Doppler result, the participant was not to be hospitalised during pregnancy for intrauterine growth retardation. In case of a red Doppler result, the management of the participant was left to the clinician in charge. In case of an orange Doppler result, the examination was repeated within three to four days. The same consequences were applied to the results of these extra examinations. Meanwhile, the policy was not to hospitalise for intrauterine growth retardation.

In the control group, information on the quality of the umbilical blood flow velocities was not revealed to the doctor or midwife in attendance. Participants in the control group received conventional clinical management for suspected IUGR. To establish the content of this 'routine clinical policy' in suspected IUGR, a questionnaire was presented to obstetricians in the university hospitals in the Netherlands (Nienhuis 1994). The results of the questionnaire are described in chapter 7.

Theoretically, participating clinicians could either be strongly requested or forced to comply with the study protocol. The choice was discussed with the epidemiologist A.R. Feinstein (Yale University School of Medicine, New Haven, USA) before the start of the trial. Requesting compliance was thought to entail a greater risk of protocol deviations, but enforcement might involve other unknown effects. As the objective of the study was to assess the effectiveness of the application of Doppler ultrasound in clinical practice, the former was decided upon. 


\subsection{Follow-up}

\subsubsection{Costs}

The perspective of the costs analysis is of great importance in an economic evaluation. If the results of the study can potentially influence a whole population, the perspective of the society is the perspective of choice (Rutren 1993). In this perspective, all costs and benefits involved, must be assessed. This is in contrast with the situation whereby the perspective of the hospital is chosen. A major proportion of personnel and material is present already, and the study will only lead to small shifts in their application. In that case, these costs will only change marginally. In this trial, the national perspective was chosen and the integral costs were assessed.

True costs are often not known. The accuracy needed for the cost-analysis partly depends on the results. If no quantitative differences are found in the number of medical procedures, a rough estimate of costs suffices. In such an assessment, tariffs can be used in the calculations rather than true costs. If the number of medical procedures differs significantly between the two groups, true costs need to be established. Medical procedures which are relatively inexpensive have little influence on the total costs. Therefore, the minute assessment of such costs is superfluous.

For the cost-analysis, medical procedures of all participants were tallied from the database of the AZM financial administration. The analysis concentrated primarily on the number of perinatal hospital days, as these formed the greatest cost factor, outweighing less expensive procedures such as CTG monitoring, ultrasound scans, or outpatient visits. Reassurance obtained by Doppler ultrasound examinations could potentially prevent hospital admission in low risk cases. A graphic presentation of the expected results of the trial is shown in figure 4.1. Maternal hospitalisation during pregnancy is expected to be reduced by not admitting cases of genetically smallness. As these cases are low risk, neonatal hospitalisation will theoretically remain unchanged.

If this intervention would be applied on a national scale, the expected savings and costs were estimated to be as in table 3.4. The method used in the calculations below is described in full elsewhere (Ament 1995).

Savings comprise the cost of hospital days prevented in those cases suspected of growth retardation who are at risk for hospitalisation, but who are in fact genetically small. Savings are calculated as shown in the formula below. These savings also depend on the sensitivity of Doppler ultrasound examinations in the detection of genetic smallness.

$$
\begin{aligned}
\text { Savings }= & n_{\text {births }} \times \%_{\text {sIUGR }} \times \%_{\text {generically small }} \times \%_{\text {hospitalised }} \\
& \times n_{\text {hospital days }} \\
& \times \text { costs hospital day } \times \text { sensitivity }
\end{aligned}
$$

whereby: $n_{\text {births }}=$ total number of singleton births; $\%_{\text {sIUGR }}=$ percentage suspected of growth retardation; \% hospitalised $=$ the percentage of cases hospitalised for suspected IUGR; 
$\%_{\text {geneticallysmall }}=$ percentage of suspected cases which are in fact genetically small; $n_{\text {hospital }}$ $d_{a y s}=$ mean number of hospital days for IUGR; costshospital day = costs of one hospital day; sensitivity = sensitivity with which Doppler ultrasound measurements can identify genetic smallness

The costs of the Doppler intervention (Costs) were inferred from the tariffs of a routine ultrasound examination plus the additional costs of equipment needed for a Doppler examination (costsDoppler).

Costs of the intervention constitute the product of Doppler intervention costs and the number of cases suspected of growth retardation in which this procedure is applied:

$$
\text { Costs }=n_{\text {births }} \times \%_{\text {sIUGR }} \times \text { costs } \text { Doppler } \times n_{\text {Doppler }}
$$

whereby: costs Doppler $=$ costs of one Doppler examination; $n_{\text {Doppler }}=$ mean number of Doppler examinations per case

The net savings equals savings minus costs:

$$
\text { Net savings = Savings - Costs }
$$

Table 3.4 shows the estimates made of the net savings if the Doppler ultrasound measurements based intervention would be implemented in all hospitals in the Netherlands. Data for this estimate were collected from several sources.

Firstly, the Central Bureau of Statistics collects data on all aspects of the Dutch population.

Secondly, routine clinical management of suspected IUGR was assessed by a questionnaire held among $80 \%$ of obstetricians in university hospitals in the Netherlands (Nienhuis 1994). The results of the questionnaire are described in full in chapter 7, but the results which are used in this and the following chapters are briefly presented here. - From annual estimates of the obstetricians, IUGR was suspected in approximately $12 \%$ of singleton pregnancies at university hospital outpatient departments.

- Approximately 69\% of these patients is hospitalised for suspected IUGR.

Thirdly, the diagnosis related groups (DRG) system at the AZM, allots codes to specific disease states and enables medical procedures for each state to be tallied (Voss 1993). The average duration of hospitalisation for IUGR was 15 days.

The following data were used in the calculations:

- In 1991 there were 193,780 singleton deliveries in the Netherlands (CBS 1993). The annual population at risk would consist of $12 \%$ of this total (questionnaire). Therefore, 23,254 pregnancies are suspected annually of IUGR in the Netherlands.

- Approximately $69 \%$ of these cases is hospitalised for suspected IUGR (questionnaire). 
- The rate of a hospital day is approximately Dfl.1,000 (Dfl. = Durch guilders).

- The average number of hospital days for suspected IUGR at the AZM was 15 days according to the DRG.

- The cost of a Doppler examination was derived from that of a specialised ultrasound examination and was estimated at Dfl.180.

- An average of five Doppler examinations was estimated to be required per patient.

- Estimates with a high degree of uncertainty were the prevalence of genetic smallness and the sensitivity of Doppler ultrasound testing for genetic smallness. The prevalence was arbitrarily estimated at $30 \%$ as no well founded assessment of this item could be derived from the literature. The sensitivity with which Doppler ultrasound measurements could detect genetic smallness, defined as the absence of postnatal catch-up growth, was tentatively set at $50 \%$.

IUGR is suspected annually in 23,254 pregnancies. These cases have a hospitalisation risk of $69 \%$, of which an estimated $30 \%$ is at low risk. Average duration of hospitalisation is 15 days, at $f 1.1,000$ a day. The sensitivity of Doppler ultrasound measurements in the identification of the fetus not at risk is $50 \%$. The savings on hospital costs would equal:

Savings $=23,254 \times 30 \% \times 69 \% \times 15 \times 1,000 \times 50 \%=$ Dfl. $36,101,835$

The intervention would require an average of five Doppler examinations to be performed on cases at risk at Dfl.180 per examination. The estimated costs of a Doppler based hospitalisation policy would equal:

$$
\text { Costs }=23,254 \times 180 \times 5=\text { Dfl. } 20,928,600
$$

This results in estimated net savings of:

$$
\text { Net savings }=36,101,835-20,928,600=\text { Dfl. } 15,173,235
$$

The above factors and formulas are used to calculate the net savings on a national basis as shown again in table 3.4 .

Theoretically, on a national basis the sizable sum of 15 million Dutch guilders could anually be saved by implementing Doppler ultrasound measurements as a reassurance test in suspected IUGR.

To estimate the influence of the above factors on outcome, they were varied with a sensitivity analysis. The accuracy of the factors 'percentage genetically small fetuses' (\%IUGR) and 'average number of hospital days for IUGR' (nhospital days) was found to be especially of influence on the estimate in table 3.4 (Ament 1995).

The costs of false positive and false negative diagnosis must also be taken into account. A false positive diagnosis in a genetically small infant may lead to unnecessary hospitalisation. However, without the Doppler based clinical policy the chance of hospitalisation 
Table 3.4

Estimated benefit of the application of Doppler ultrasound measurements as a reassurance test in the Necherlands

\begin{tabular}{lcc}
\hline Cost parameters & Estimates & Number of cases \\
\hline singleton deliveries & $100 \%$ & 193,780 \\
suspected IUGR & $12 \%$ & 23,254 \\
hospitalised for sIUGR & $69 \%$ & 16,045 \\
generically small & $30 \%$ & 4,813 \\
mean number of hospital days & 15 & \\
costs hospital day & Dfl. 1,000 & \\
costs Doppler examination & Dfl. 180 & \\
mean number of Doppler examinations & 5 & \\
sensitivity of Doppler examination & $50 \%$ & \\
& & \\
Savings & Dfl. $36,101,835$ & \\
Costs & Dfl. $20,928,600$ & \\
Net savings & Dfl. $15,173,235$ \\
\hline
\end{tabular}

${ }^{1}$ annual number of singleton births over 24 weeks gestational age in the Netherlands (CBS 1993); sIUGR = suspected IUGR; DF. = Dutch guilders

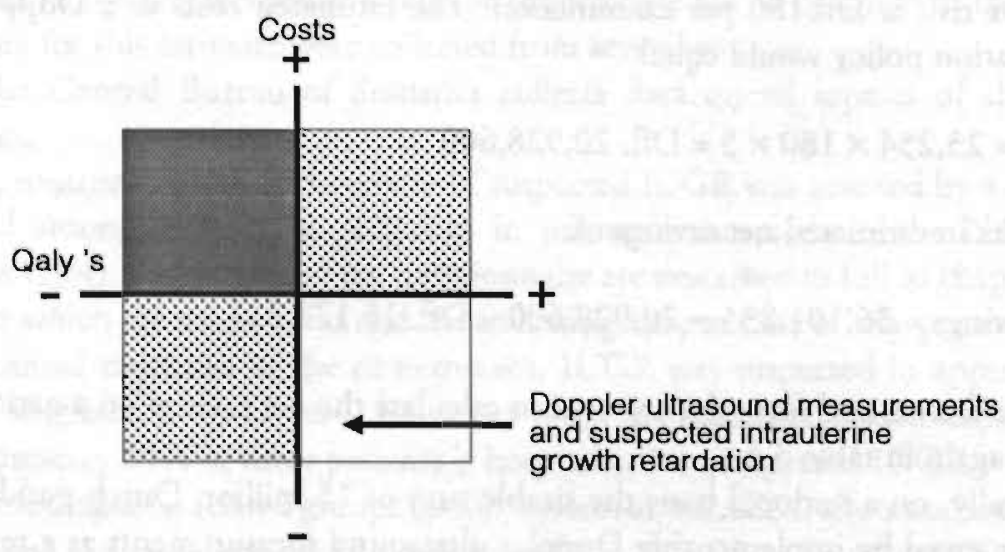

Figure 3.5

Costs and quality adjusted life years (Qaly's) 
would be even higher. The overall expected effect is a reduction of costs compared to the conventional policy in suspected IUGR. Moreover, such false positive tests occur mainly when the prior probability of IUGR and the specificity of the test are low. The former is unlikely in a preselected high risk population. The occurrence of false negative tests cannot as yet be quantified.

Besides costs, the benefit to patients should also be taken into account. This aspect can be expressed in Qaly's (quality adjusted life years). It is not likely that the Doppler based intervention will reduce Qaly's. A test which reassures the clinician in charge, will generally reassure the patient. Moreover, the intervention is intended to reduce costs by reducing unnecessary hospitalisation in case of genetic smallness. Theoretically, this has no detrimental effect on perinatal outcome, as the perinatal risk in these cases is low. In addition, patients often experience hospitalisation itself as stressful (Kramer 1986, White 1984), so that reduced hospitalisation is more likely to have a positive effect on Qaly's. The expected result of the trial described in this thesis is illustrated in figure 3.5. The axis show the relation between costs and Qaly's. Many new techniques are implemented to improve the quality of life. This often leads to an increase in costs. The white area indicates an intervention that both diminishes costs and increases Qaly's. According to our hypothesis, the Doppler based intervention will reduce costs without affecting Qaly's. Of the four types of cost appraisal (Drummond 1987), cost analysis was the analysis of choice for this trial, as the effects were expected to be similar in the two groups and the difficult task of calculating Qaly's could be avoided.

\subsubsection{Perinatal outcome}

To evaluate the effect of Doppler ultrasound as reassurance test in suspected IUGR, obstetric management and perinatal outcome measures were recorded. Recorded items correspond to those presented in the meta-analysis of the RCTs (section 2.6). These included the onset and mode of delivery, birth weight, and gestational age at birth. Birth weight centiles were calculared by a program (P. Kurver, Buro Medische Automatisering) based on the Kloosterman birth weight centiles (1970).

\subsubsection{Neurological development}

That the Doppler based intervention was not detrimental to the infants concerned, was tested by repeated assessment of neurological development. Neurological development was assessed by an experienced child neurologist, who was unaware of the Doppler results. Examinations were performed after delivery, and at 6 and 9 months.

Between 3-8 days after term delivery, the neonates were examined according to the method developed by Prechtl (Prechtl 1977, appendix 1). The full Prechtl examination was performed and the results were noted on the accompanying summary form. Age specific neurological examinations (Touwen 1984) were performed at the corrected ages of 6 and 9 months \pm 2 weeks (appendix 2). 
Infants were examined on the neonatal ward or in the outpatient department. The examinations were performed in a warm room and the examination time was 20 to 30 minutes. The overall result of each examination was categorised as being 'normal', 'suspect', or 'abnormal'.

In preterm infants, the use of chronological age could overestimate a possible delay in neurological development, while correction for the GA could underestimate such an effect. According to the literature, correction of the examination date for gestational age was advised in preterms in the first year after delivery (Brandt 1991, den Ouden 1991, Lems 1993). Preterm infants were, therefore, examined within 8 days after reaching term ( 37 completed weeks of gestation $=259$ days) and further examination dates were corrected for the GA.

\subsubsection{Postnatal growth}

In the review of the literature, it was hypothesised that the occurance of postnatal catch-up growth might be an adequate retrospective standard for IUGR (section 2.7.1). A significant upward change of the deviation from the mean with advancing age would suggest catch-up growth. A deviation in size from the mean for age can be expressed by using standard deviation scores (SDSs) (Preece 1986).

$$
S D S=\frac{\text { measurement }- \text { mean for age }}{S D \text { for age }}
$$

On the basis of the literature, catch-up growth can be defined as an upward shift of one or more SDSs in the first six months after delivery (Davies 1980, Scott 1982).

In order to be able to calculate SDSs, reference values for the mean and the standard deviations of anthropometric growth parameters in unrestrained growth were required for both preterm and term infants. The use of anthropometric reference curves allows standardisation of anthropometric measurements for age and sex. Measurements taken at different points in time can then be more easily compared. However, a continuous anthropometric reference for height, $\mathrm{HC}$, and weight from extrauterine viability to the first year after delivery is not provided by the literature.

However, if mean height, head circumference, and weight of the published studies presented in table 2.1 are plotted against age, the general shape of the relation of these parameters with age emerges (section 2.1, figures 2.1-2.3). Although the course of height and head circumference with gestational age is shown to be gently $S$-shaped, the exact shape is not known. As was discussed in section 2.1, height and head circumference are shown to correlate linearly with gestational age for a major part of pregnancy. Although the relation of weight with gestational age progresses exponentially from conception, a linear relation was established between 196 days GA and 140 days after delivery ( 417 days). To analyse the anthropometric data of the infants participating in the RCT, reference growth curves were constructed from the reviewed anthropometric studies in table 2.1. Objections against such constructed reference curves may be alleviated by keeping in 
mind that the goal of the analysis was not to describe fetal and neonatal growth exactly, but to compare the quality of postnatal growth between the two study groups. Anthropometric reference curves for both mean and standard deviation for height, head circumference, and weight were constructed as follows.

The basis of the postnatal 'growth' reference was provided by the widely used Dutch cross-sectional references for height, weight, and head circumference in healthy infants of multipara (Roede 1985). These data corresponded well to the longitudinal growth curves of other Western European countries (figures 2.1-2.3). However, the earliest reference data in the Dutch study start at two weeks (294 days) for $\mathrm{HC}$ or four weeks (308 days) for weight and height after delivery. Therefore, reference data at 280 days of gestation (the estimated date of birth) needed to be established.

Extrapolation was realised by fitting the Dutch study and the reviewed longitudinal studies (table 2.1) with a spline smoother programme (S-plus, Statistical Sciences Europe, Oxford) and reading off the outcome at 280 days. The underlying assumption was that optimal growth is not constrained at the end of pregnancy. This data point was subsequently added to the data of the Dutch study. Data from the Dutch study, including the data point at 280 days, were fitted again to obtain a mathematical description of the relation of the mean of the anthropometric parameters with age, from the estimated date of birth to the first year of extrauterine life.

With regard to the reference of growth before the estimated date of birth, fetal size at conception was theoretically taken to be zero and optimal growth was considered to be unconstrained in principle. The occurrence of a downward slope at term can be interpreted as a restriction of unconstrained growth, and can, therefore, be disregarded. In the constructed reference curves, mean gain of height and head circumference was taken to be linear from conception (day 14 with size $=0$ ) and mean weight gain was linear from 196 days of gestation to the estimated time of delivery ( 280 days), in accordance with the literature. This linear approximation offered clarity and prevented false precision. Mean weight and SD at 196 days were estimated from the reviewed early cross-sectional studies (table 2.1).

Lastly, the reference standard deviations were added. Standard deviations from 280 days of gestation to the age of one year were fitted from the combined postnatal longitudinal growth studies and the cross-sectional Dutch study. Before the estimated day of delivery, the distribution of standard deviations with age is notably wide. This is shown in figure 3.6 for the SDs of height obtained from the reviewed studies. Data points with less than 20 participating subjects at that GA were excluded.

Similar results were found for head circumference and weight. The variation originated mainly from the cross-sectional post mortem studies (figure 3.7). Because abortion or intrauterine fetal death is more likely be associated with growth disturbances, such studies are not applicable as a reference for optimal growth.

Theoretically, the standard deviation at conception is zero. The SDs up to 280 days of gestation could be larger, similar, or smaller than the known SDs after delivery. Figure 


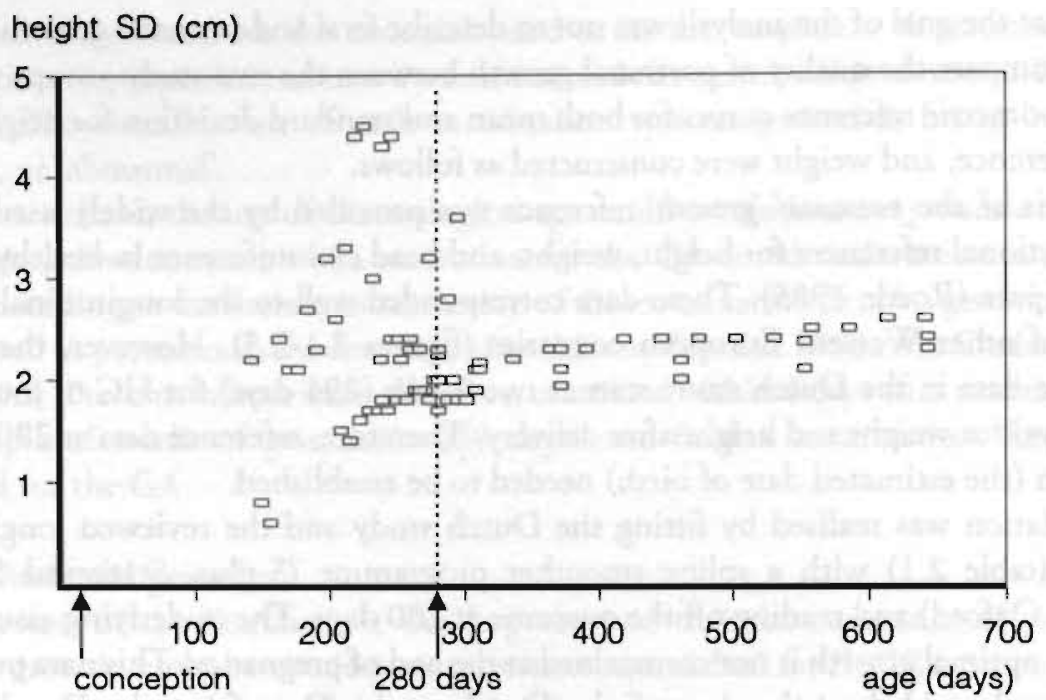

Figure 3.6

Standard deviations of height with age (reviewed studies table 2.1, boys).

height SD $(\mathrm{cm})$

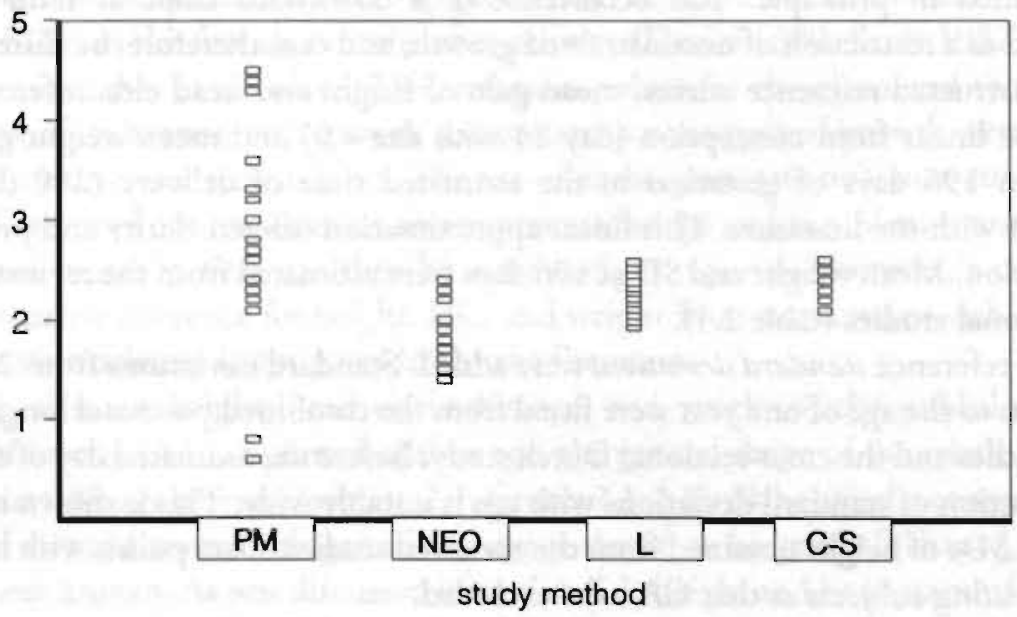

Figure 3.7

Standard deviations of height derived from different study mechodologies (reviewed studies table 2.1 , boys).

$\mathrm{PM}=$ cross-sectional measurements obtained at post mortem, NEO=cross-sectional anthropometric measurements of neonates after delivery, L=longitudinal, C-S = cross-sectional 
3.6 suggests that they are larger. However, autopsy studies do not provide optimal data for normal size for the reasons stated. Influences leading to deviation of growth from normal will lead to increasing variation in size with gestational age (Stembera 1985). How much of this effect can be attributed to physiology and how much to pathology is unknown. If the SDs were to remain constant during the latter part of the fetal period, a bend is needed to connect the SD in this period with the zero SD at conception. This seems artificial. The final option of a proportional relation between age and SDs before 280 days seems the most logical and physiological choice. Lines were drawn for height $\pm 1 S D$ and head circumference $\pm 1 S D$ from conception (age $=14$ days, size $\pm 1 S D=0$ ) to the beginnings of the $\pm 1 S D$ curves at 280 days. The functions of these linear references $y=a+b x$ were calculated by entering the two known data points at conception $(14,0)$ and at 280 days $(280$,calculated $y)$ into the formula:

$$
\mathrm{b}=\left(\mathrm{y}_{x=280}-0\right) /(280-14) \quad \mathrm{a}=-\mathrm{b} \times 14
$$

As the course of weight with gestational age is considered to be linear from 196 days of gestation, mean weight and SD at 196 days were calculated from the respective fits of all the reviewed data. Lines were drawn between these values and the results at 280 days. Their functions were again assessed by entering the known data points:

$$
\mathrm{b}=\left(\mathrm{y}_{x=280}-\mathrm{y}_{x=196}\right) /(280-196) \quad \mathrm{a}=\mathrm{y}_{x=196}-\mathrm{b} \times 196
$$

Since growth curves in the literature were found to deviate between the sexes from late gestation onwards (Kloosterman 1970, Ounsted 1982b), separate curves were constructed for each sex. The constructed reference curves are shown in figures 3.8 to 3.10. The fits of the reference curves are given in appendix 3.

Anthropometric measurements in the RCT described in this thesis, were performed on all participants at the AZM. The measurement frequency was every two weeks during the first three months after delivery, and monthly until the corrected age of 9 months. Weight (W) of the fully undressed infant was determined in grams.

Crown to heel length or height $(\mathrm{H})$ was measured with the aid of an instrument based on the Harpenden infantometer. The instrument consisted of a horizontal board and a smoothly moving footboard with a built-in digital counter from which the height could be read to the nearest millimetre. The child was placed on the measuring board and an assistant held the child's head firmly in the so called 'Frankfurter plane' whereby the lower borders of the orbits were in the same plane as the external auditory meati. The infants legs were straightened and the ankles were kept at right angles while the footboard was pulled firmly against the child's heels.

An instrument for measuring head circumferences ( $\mathrm{HC}$ ) was developed at Mastricht (Gerver 1990). The tape of the instrument was placed over the most extending point of the occiput and just above the ridges of the eye brows and tightened until the instrument indicated a standard stress. This was then repeated with a plastic disc with a known 


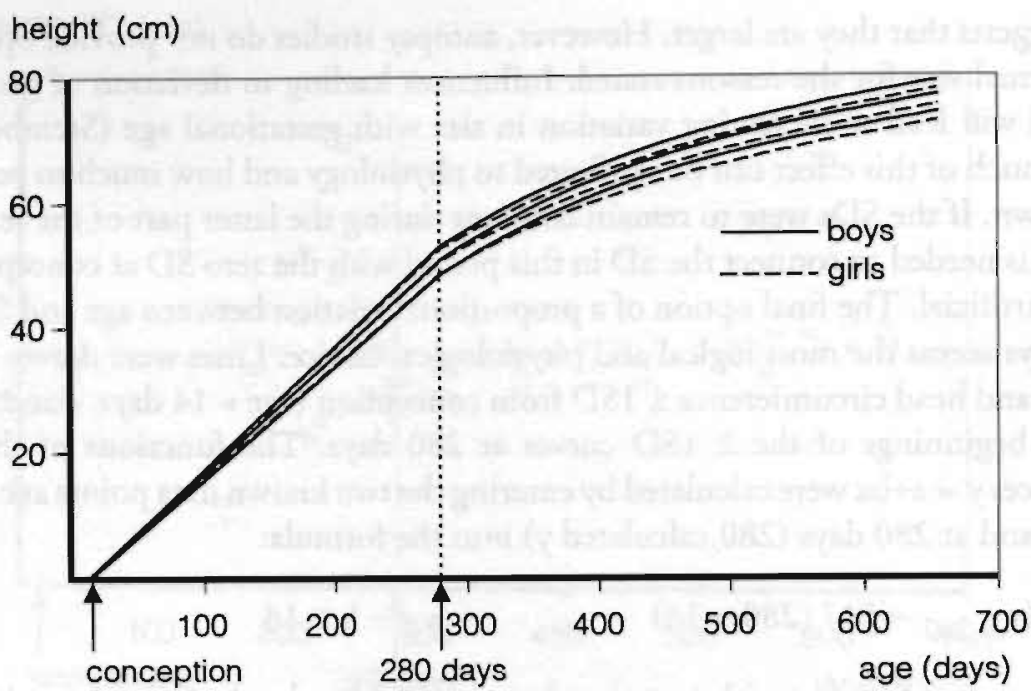

Figure 3.8

Constructed reference curve for height (mean $\pm 1 S D$ )

$\mathrm{HC}(\mathrm{cm})$

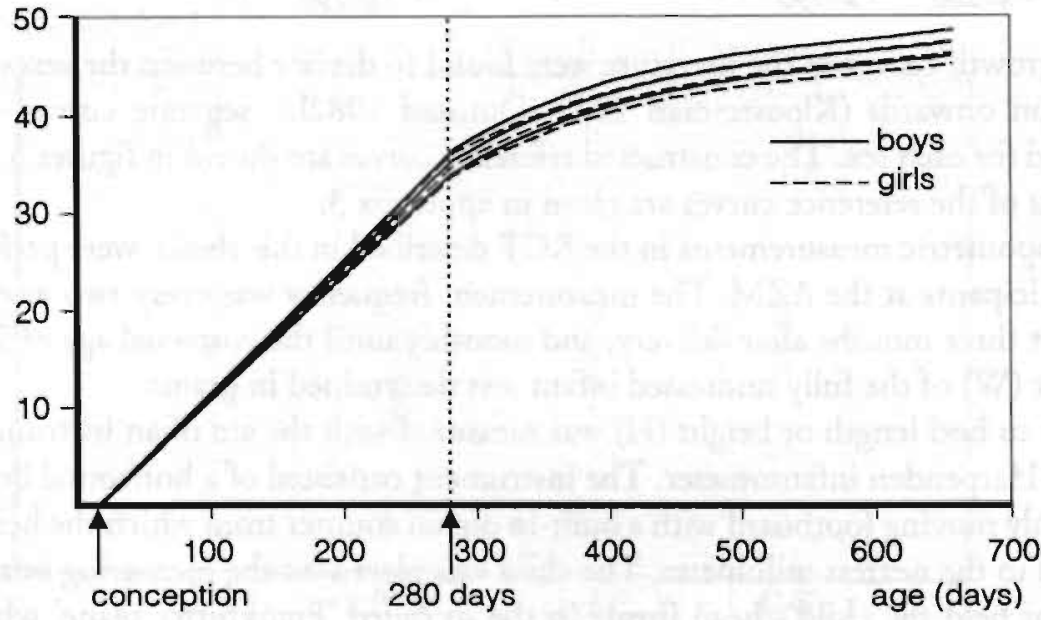

Figure 3.9

Constructed reference curve for head circumference (mean $\pm 1 S D$ )

circumference until the same stress was reached. The difference between the circumference of the infant's head and that of the disk was displayed on the digital counter. This difference was then added to or subtracted from the circumference of the reference disk to obtain the infants $\mathrm{HC}$ in millimetres. 
weight $(\mathrm{kg})$

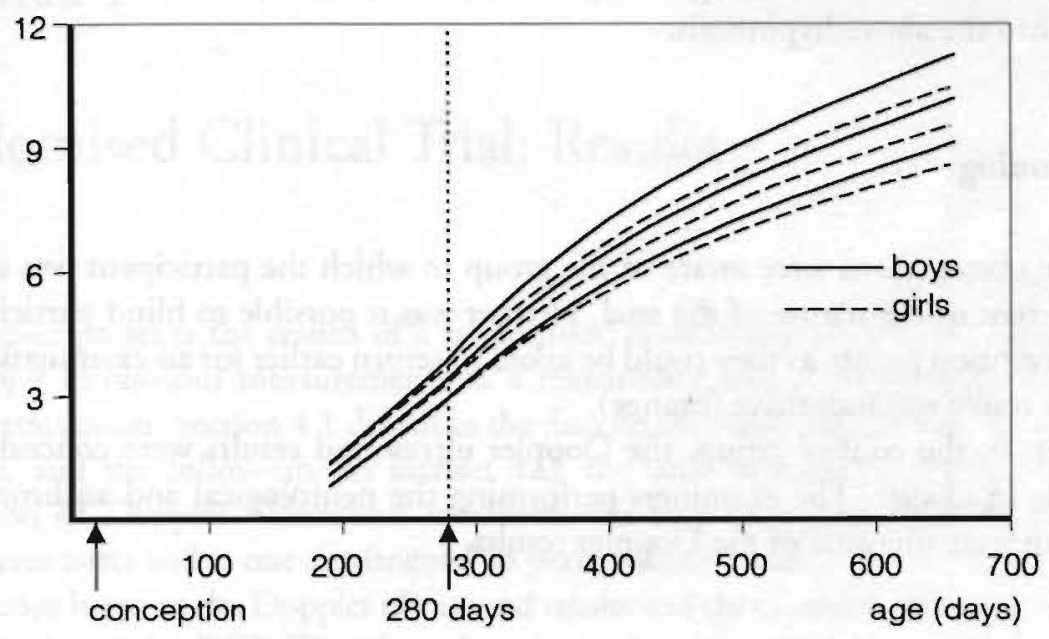

Figure 3.10

Constructed reference curve for weight (mean $\pm 1 S D$ )

\subsection{Study size}

Doppler ultrasound measurements have gradually been implemented in obstetrics without their effectivity having been clearly proven. This was previously shown in the review of the randomised clinical trials on the application of Doppler ultrasound into clinical practice (section 2.5.3). Doppler ultrasound measurements had not been used in clinical decision making at the Department of Obstetrics of the University Hospital of Maastricht, at the time the protocol was written. It was thought that Doppler techniques should not be introduced into the patient care of the department without testing its effectivity. Ultimately, such clinical trials should clarify whether Doppler ultrasound should be used in clinical decision making.

It was calculated that sizable savings could be gained by applying Doppler ultrasound measurements as reassurance test in suspected IUGR (table 3.4). Used in this way, the group suspected of IUGR could be cleared of cases of genetic smallness by the finding of normal Doppler ultrasound values in the umbilical artery. According to our hypothesis, hospitalisation during pregnancy would be reduced only in cases of genetic smallness. Postnatal costs, due to extra neonatal hospitalisation for instance, were therefore not likely to undo the savings made prenatally. Taking the potential savings into account, this hypothesis seemed worth an investigation.

In our clinical setting, the number of eligible patients was estimated at 16 to $17 \%$ of the singleton pregnancies at the AZM outpatient clinic (table 3.1). The actual accrual rate is generally half the estimated rate (Pocock 1983). An accrual period of a study should be limited to approximately $2 \frac{1}{2}$ years. In the set period, an estimated 150 participants 
could be randomised. This logistically feasible study size was used to provide some insight into the above hypothesis.

\subsection{Blinding}

That the obstetricians were aware of the group to which the participant was assigned, was inherent in the nature of the trial. Neither was it possible to blind participants in the intervention group, as they could be asked to return earlier for an examination if the Doppler result was indecisive (orange).

However, in the control group, the Doppler ultrasound results were concealed from clinicians in charge. The examiners performing the neurological and anthropometric follow-up were unaware of the Doppler results.

\subsection{Data handling and statistical analysis}

Data were collected on data forms and entered into Dbase IV (Borland). Statistical analysis was performed with SPSS-PC+.

The results of the present RCT are described in section 4.1. Differences between the intervention and the control group are tested in section 4.2. Two means were compared with a t-test and two percentages with a $\mathrm{Chi}^{2}$-test. In all tests, $\alpha=0.05$ and $\beta=0.1$. A difference between the groups was statistically significant if $p<0.05$. Differences in outcome between the study groups are presented with the associated $95 \%$ confidence interval (CI) of that difference (Pocock 1983).

The first part of the dual hypothesis stated that the Doppler based clinical management reduces costs by reducing hospitalisation in the intervention group. It was not the object of the analysis to test whether this item differed between the study groups regardless of the direction of this difference, in which case a two-tailed test could be used. One-tailed tests were, therefore, used to test if hospitalisation rate and duration during pregnancy were significantly reduced in the intervention group in comparison to the control group. The second part of the main hypothesis concentrates on the effect that this management policy has on perinatal outcome. The direction of this effect cannot be specified in advance, although the expectation is that perinatal outcome will not be negatively effected by the management policy. Differences in outcome between the intervention and the control group, such as costs, obstetric management, perinatal outcome, neurological development, and postnatal growth, were therefore tested with a two-tailed test.

Although the relation between Doppler ultrasound results and the outcome parameters of the RCT was not a main objective, such information can potentially generate hypotheses worth studying in the future. Therefore, these comparisons were presented separately in chapter 5 . 


\section{Chapter 4}

\section{Randomised Clinical Trial: Results}

This chapter presents the results of a randomised clinical trial on the costs and effects of Doppler ultrasound measurements as a reassurance test in suspected intrauterine growth retardation. Section 4.1 describes the data on the study population, the perinatal outcome, and the follow-up. In section 4.2, the dual hypothesis is tested that the application of Doppler ultrasound measurements as a reassurance test in suspected IUGR saves costs and is not detrimental to perinatal outcome.

The relations between the Doppler ultrasound results and the outcome were not part of the main objective of this RCT. Therefore, these are explored separately in chapter 5 .

\subsection{Results}

\subsubsection{Study population}

Of the 161 women referred because of suspected intrauterine growth retardation of two or more weeks, 150 (93\%) consented to join the study. The reasons for refusal were lack of time and certainty that follow-up could not be completed because of moving out of the area. One hundred and fifty women were randomised, 74 to the intervention group and 76 to the control group. Table 4.1 shows the distribution of the two stratification criteria: gestational age (GA) at randomisation (GA $\leq 32$ weeks or $G A>32$ weeks) and smoking habits (no or yes).

Stratification guarantees an even distribution of two major prognostic factors: smoking and the gestational age at which IUGR is suspected. The aim of the randomisation is to achieve an even distribution of 'minor' prognostic factors over the two groups. The resulting characteristics of the randomisation groups at inclusion are shown in table 4.2.

A number of associated maternal medical conditions was present at inclusion, some of which were of influence on the hospitalisation policy and consequently on the cost analysis (table 4.3). The influence on hospitalisation and costs was extreme in a participant in the control group (case no. 72) whose insulin dependent diabetes mellitus (IDDM) was uneventful at the time of the inclusion, but subsequently led to an excessive number of hospital days during pregnancy. 
Table 4.1

Stratification and randomisation

\begin{tabular}{|c|c|c|c|c|c|c|}
\hline & \multicolumn{2}{|c|}{$\mathrm{GA} \leq 32$ weeks } & \multicolumn{2}{|c|}{ GA $>32$ weeks } & \multicolumn{2}{|l|}{ Total } \\
\hline & $\begin{array}{l}\text { Intervention } \\
\text { group }\end{array}$ & $\begin{array}{l}\text { Control } \\
\text { group }\end{array}$ & $\begin{array}{l}\text { Intervention } \\
\text { group }\end{array}$ & $\begin{array}{l}\text { Control } \\
\text { group }\end{array}$ & $\begin{array}{l}\text { Intervention } \\
\text { group }\end{array}$ & $\begin{array}{l}\text { Control } \\
\text { group }\end{array}$ \\
\hline non smokers & 12 & 16 & 24 & 23 & 36 & 39 \\
\hline smokers & 14 & 14 & 24 & 23 & 38 & 37 \\
\hline total number & 26 & 30 & 48 & 46 & 74 & 76 \\
\hline
\end{tabular}

Table 4.2

Comparison of intervention and control group at inclusion

\begin{tabular}{|c|c|c|c|c|c|}
\hline \multirow{2}{*}{ Intake data } & \multicolumn{2}{|c|}{ Intervention group } & \multicolumn{2}{|c|}{ Control group } & \multirow[b]{2}{*}{$\mathrm{Chi}^{2}$-test } \\
\hline & $(n=74)$ & & $(n=76)$ & & \\
\hline maternal age at LMP (years) & $29 \pm 5$ & $(19-42)$ & $28 \pm 5$ & $(19-42)$ & \\
\hline maternal height $(\mathrm{cm})$ & $167 \pm 6$ & $(150-181)$ & $165 \pm 7$ & $(145-183)$ & \\
\hline pre-pregnancy weight $(\mathrm{kg})$ & $58 \pm 8$ & $(42-80)$ & $56 \pm 9$ & $(40-80)$ & \\
\hline maternal weight gain $(\mathrm{kg})$ & $11 \pm 4$ & $(2-22)$ & $11 \pm 4$ & $(2-22)$ & \\
\hline paternal height $(\mathrm{cm})$ & $178 \pm 7$ & $(160-193)$ & $178 \pm 8$ & $(160-210)$ & \\
\hline paternal weight $(\mathrm{kg})$ & $78 \pm 11$ & $(52-110)$ & $78 \pm 11$ & $(55-104)$ & \\
\hline primipara & 34 & $(45.9 \%)$ & 43 & $(56.6 \%)$ & $p=0.19$ \\
\hline GA at diagnosis of sIUGR (days) & 230 & $(137-269)$ & 225 & $(133-269)$ & \\
\hline GA at inclusion (days) & 233 & $(137-271)$ & 233 & $(159-283)$ & \\
\hline
\end{tabular}

Results are shown as mean \pm standard deviation (SD) and range (minimum-maximum), parity as number and percentage of primipara, and GA as median and range. LMP = last menstrual period; GA = gestational age; sIUGR = suspected IUGR.

A number of maternal physical and psychological disorders became apparent during the course of the study. These disorders are presented in table 4.4. An asterisk indicates which is analysis influenced. This will be discussed further in the second part of this chapter.

In a participant in the intervention group (case no. 103), no psychological problems were apparent at the time of inclusion. She subsequently developed a psychosis which required admission to the psychiatric ward for almost five months, two of which during pregnancy. In one case of severe puerperal depression, appointments for follow-up of neurological development could not be attended. 


\section{Table 4.3}

Associated maternal disorders

\begin{tabular}{lllll}
\hline Diagnosis & Intervention group & Control group & \multicolumn{2}{l}{ Influence on analysis of: } \\
\cline { 4 - 5 } & case no. & case no. & Outcome & Costs \\
\hline donor kidney & 79 & 99,110 & $*$ & $*$ \\
IDDM & & 72 & & $*$ \\
$\begin{array}{l}\text { ulcerative colitis } \\
\text { hyperthyroidism }\end{array}$ & & 102 & $*$ & $*$ \\
multiple sclerosis & & 98 & & $*$ \\
Strumpell's syndrome & 70 & 45 & $*$ & \\
\hline
\end{tabular}

case no. $=$ participants registration number, $*$ of influence on the part of the analysis indicated. IDDM = insulin dependent diabetes mellitus, Strumpell's syndrome = disease of the motor system

\section{Table 4.4}

Maternal disorders diagnosed during the course of the study

\begin{tabular}{lllll}
\hline Diagnosis & Intervention group & Control group & \multicolumn{2}{l}{ Influence on analysis of: } \\
& case no. & case no. & Costs & Neuro \\
\hline $\begin{array}{l}\text { psychosis } \\
\text { pregnancy induced diabetes }\end{array}$ & 103 & & $*$ & $*$ \\
puerperal depression & 16 & 2 & & $*$ \\
\hline
\end{tabular}

* of influence on the part of the analysis indicated.

\section{Table 4.5}

Doppler ultrasound results

\begin{tabular}{|c|c|c|c|c|}
\hline Doppler result & Category & $\begin{array}{l}\text { Clinical policy according to } \\
\text { protocol in the intervention } \\
\text { group }\end{array}$ & $\begin{array}{l}\text { Intervention group } \\
(\mathrm{n}=74)\end{array}$ & $\begin{array}{l}\text { Control group } \\
(n=76)\end{array}$ \\
\hline green & normal & no hospitalisation & $42(56.7 \%)$ & $45(59.2 \%)$ \\
\hline orange & indecisive & $\begin{array}{l}\text { no hospitalisation, } \\
\text { repeat examination }\end{array}$ & $9(12.2 \%)$ & $7(9.2 \%)$ \\
\hline red & raised & determined by clinician & $15(20.3 \%)$ & $18(23.7 \%)$ \\
\hline (zero/reversed flow) & raised & determined by clinician & $8(10.8 \%)$ & $6(7.9 \%)$ \\
\hline
\end{tabular}


Table 4.6

Comparison of obstetric management and perinatal outcome

\begin{tabular}{|c|c|c|c|c|c|}
\hline \multirow{2}{*}{ Perinatal ourcome } & \multicolumn{2}{|c|}{ Intervention group } & \multicolumn{2}{|c|}{ Control group } & \multirow[b]{2}{*}{ Chi ${ }^{2}$-test } \\
\hline & $(\mathrm{n}=74)$ & & $(\mathrm{n}=76)$ & $(\%)$ & \\
\hline boys & 43 & $(58.1 \%)$ & 28 & $(36.8 \%)$ & \\
\hline girls & 31 & $(41.9 \%)$ & 48 & $(63.2 \%)$ & $\mathrm{p} \equiv 0.01$ \\
\hline spontaneous onset of delivery & 51 & $(68.9 \%)$ & 51 & $(67.1 \%)$ & \\
\hline induction of labour/elective $\mathrm{CS}^{\mathrm{I}}$ & 21 & $(28.4 \%)$ & 21 & $(25.0 \%)$ & \\
\hline induction of labour not for IUGR ${ }^{2}$ & 2 & $(2.7 \%)$ & 6 & $(7.9 \%)$ & \\
\hline spontaneous delivery & 63 & $(85.1 \%)$ & 57 & $(75.0 \%)$ & \\
\hline elective CS & 6 & $(8.1 \%)$ & 4 & $(5.3 \%)$ & \\
\hline emergency CS & 2 & $(2.7 \%)$ & 7 & $(9.2 \%)$ & \\
\hline forcipal extraction & 3 & $(4.1 \%)$ & 7 & $(9.2 \%)$ & \\
\hline vacuum extraction & 0 & (0) & 1 & $(1.3 \%)$ & \\
\hline IUFD (due to defects ${ }^{3}$ ) & $1(0)$ & $(1.4 \%)$ & $3(1)$ & $(3.9 \%)$ & \\
\hline neonatal death (due to defects ${ }^{3}$ ) & $1(1)$ & $(1.4 \%)$ & $0(0)$ & $(0 \%)$ & \\
\hline deviant CTG $^{4}$ & 30 & $(40.5 \%)$ & 27 & $(35.5 \%)$ & \\
\hline breech presentation & 3 & $(4.1 \%)$ & 14 & $(18.4 \%)$ & $\mathrm{P}=0.01$ \\
\hline preterm birth & 13 & $(17.6 \%)$ & 13 & $(17.1 \%)$ & \\
\hline birth centile $<\mathrm{P} 2.3$ & 18 & $(23.3 \%)$ & 13 & $(17.1 \%)$ & \\
\hline birth centile $<$ P10 & 36 & $(48.6 \%)$ & 42 & $(55.3 \%)$ & \\
\hline birth weight $\leq 2500$ grams & 29 & $(39.2 \%)$ & 24 & $(31.6 \%)$ & \\
\hline GA at birth (days) & 273 & $(200-299)$ & 275 & (184-295) & \\
\hline birth weight (grams) & 2737 & $(640-3975)$ & 2695 & $(480-3790)$ & \\
\hline Ist test to delivery interval (days) & 38 & $(0-135)$ & 36 & $(4-110)$ & \\
\hline
\end{tabular}

Results are shown as number and percentage, or as median and range.

$\mathrm{CS}=$ Caesarean section; IUFD = intrauterine fetal death; ${ }^{1}$ includes prostaglandin or oxytocin induction of labour and elective Caesarean section (CS) for IUGR. Does not include induction of labour for IUFD or chemical stimulation because of ruptured membranes without contractions; ${ }^{2}$ indications intervention group: intrauterine fetal death, Strumpell's syndrome indications control group: three IUFDs, post term pregnancy, bleeding, ulcerative colitis; ${ }^{3}$ includes chromosomal and/or congenital defects; ${ }^{4}$ late deceleration or bradycardia on the cardiotocogram

\subsubsection{Doppler ultrasound measurements}

The median study period from inclusion to delivery was 38 days in the intervention group (range 0-135 days) and 36 days in the control group (range 4-110 days). An average of 5.9 Doppler measurements was performed in the intervention group (range 1-15) and 5.7 in the control group (range 1-16). 
The Doppler ultrasound results are shown in table 4.5. During the study period, the results were only known to the clinicians in the intervention group. The results of the control group were concealed. To recapitulate from section 3.2.6, the Doppler result was labelled green if the PI < mean+1SD of the reference established by van Vugt (figure 3.2 ), orange when mean $+1 S D \leq P I \leq$ mean $+2 S D$, and red if PI $>$ mean $+2 S D$. The latter category also included cases with diastolic zero flow or reversed flow. These are shown separately in table 4.5 to enable meta-analysis. The conclusive Doppler ultrasound result shown, is the 'worst' result (a raised PI) obtained during the study period, because that result could lead to hospitalisation. The advised clinical policy linked to that Doppler ultrasound result in the intervention group is also shown. Doppler results were not corrected for fetal heart rate, as no measurements were made outside the physiological range of 120-160 beats per minute (Legarth 1989).

\subsubsection{Perinatal outcome}

Table 4.6 shows a general overview of the perinatal clinical outcome in the two groups. Statistical analysis of these data is presented in section 4.2.2.

There were four intrauterine fetal deaths (IUFDs) during the study period, one in the intervention group, three in the control group. Multiple abnormalities were found in one IUFD in the control group, which was subsequently excluded from further analysis. One neonatal death in the intervention group was due to a severe cardiac anomaly and was therefore excluded. The clinical details are shown in table 4.7. A short description of each case is given.

Table 4.7

Intrauterine fetal deaths (IUFD)

$\begin{array}{llll}\text { Group } & \begin{array}{l}\text { Case } \\ \text { no. }\end{array}\end{array}$ Doppler $\begin{aligned} & \text { GA } \\ & \text { (days) }\end{aligned} \begin{aligned} & \text { Weight } \\ & \text { (grams) }\end{aligned}$ Centile Defects Cause

\begin{tabular}{|c|c|c|c|c|c|c|c|}
\hline Intervention & 96 & zero flow & 202 & 750 & $<\mathrm{P} 2.3$ & no & $\begin{array}{l}\text { IUFD: placental } \\
\text { insufficiency }\end{array}$ \\
\hline Intervention & 22 & normal & 285 & 2580 & $<\mathrm{P} 2.3$ & yes & $\begin{array}{l}\text { neonatal death: VSD } \\
\rightarrow \text { excluded }\end{array}$ \\
\hline Control & 19 & zero flow & 184 & 480 & $<\mathrm{P} 2.3$ & no & $\begin{array}{l}\text { IUFD: placental } \\
\text { abruption }\end{array}$ \\
\hline Control & 29 & normal & 207 & 1060 & P10-P25 & yes & $\begin{array}{l}\text { IUFD: multiple defects } \\
\rightarrow \text { excluded }\end{array}$ \\
\hline Contro] & 110 & zero flow & 278 & 2700 & PS & no & $\begin{array}{l}\text { IUFD: not optimal } \\
\text { antenatal care in patient } \\
\text { with donor kidney }\end{array}$ \\
\hline
\end{tabular}

VSD = ventricular septal defect 
Intervention group case no. 96: IUFD

29 years old GII PO AI

Medical history: mild mitral valve regurgitation

Obstetric history: spontaneous abortion at $71 / 2$ weeks GA followed by curettage

Pregnancy: A lag in fundal height and hypertension of $135 / 100$ was found at a routine visit to the outpatient clinic at $245 / 7$ weeks GA. A raised PI (red) was found in the umbilical artery. During admission, diastolic flow fell to zero and the CTG showed insufficient variability. Fetal weight could not be reliably assessed, due to oligohydramnios, but was estimated at $<500$ grams. After being fully informed by the neonatologists, the parents decided to abstain from obstetric intervention. At $285 / 7$ weeks, IUFD occurred.

Delivery: Nalador induction

Placenta: 135 grams

Obduction: no congenital defects

Intervention group case no. 22: neonatal death

21 years old GI P0 A0

Medical history: -

Obstetric history: -

Pregnancy: Lag of two weeks in fundal height at $323 / 7$ weeks GA. Otherwise uncomplicated pregnancy.

Delivery: uneventful delivery, Apgar score at five minutes: 10 , umbilical artery $\mathrm{pH}=7.11$.

Placenta: 290 grams

Neonate: No neonatal problems. At the age of three months, a large ventricular septal defect (VSD) was detected with pulmonary stenosis and moderate aorta stenosis. The infant died at the age of four months after surgical correction of the defect.

\section{Control group case no. 19: IUFD}

31 years old GI PO A0

Medical history: -

Obstetric history: -

Pregnancy: The patient reported to the outpatient clinic with blood loss and contractions at $246 / 7$ weeks GA. There was zero flow from the first Doppler examinarion, but this was not conveyed to the clinician in charge, in accordance with the study protocol. The CTG showed variable decelerations. The patient was admitted to hospital under suspicion of a partial placental abruption. Because of the expected low fetal weight and the immaturity, tocolysis was started. At 26 weeks GA, IUFD was diagnosed.

Delivery: Nalador induction

Placenta: 140 grams, no macroscopic or microscopic explanation for the IUFD

Obduction: no defects

Control group case no. 29: IUFD

27 years old GII PI A0

Medical history: -

Obstetric history: term delivery of SGA infant 
Pregnancy: uneventful pregnancy up until $291 / 7$ weeks GA, referred to the study because of a three week lag in fundal height. The patient consented to participate at the first appointment. At this examination, there was oligohydramnios and a Doppler result within the normal range, which was withheld from the clinician in charge, in accordance with the study protocol. At the outpatient clinic five days later, there was loss of amniotic fluid stained with meconium and there was no heart action.

Delivery: spontaneous onset and breech delivery

Placenta: 220 grams, signs of intrauterine infection

Obduction: macerated neonate with multiple anomalies: microcephaly, micrognathia, high palate, syndactyly of the fourth and fifth toes on both sides, and small cystic kidneys without ureters, normal 46,XX karyotype

\section{Control group case no. 110: IUFD}

27 years old GI PO A0

Medical history: kidney transplantation after glomerulosclerosis, multiple congenital defects, antihypertensive medication

Obstetric history: -

Pregnancy: The patient was referred to the study at 29 weeks GA. At the intake, the Doppler results were in the normal range, but at further examinations, the PIs were raised ('red', but with diastolic forward flow). All the Doppler results were withheld from the clinician in charge, in accordance with the study protocol. The patient was not hospitalised during pregnancy. The condition of the donor kidney remained stable. At the Doppler examination at 40 weeks GA, no heart action could be found. This IUFD might have been prevented if the Doppler values had been known.

Delivery: induction of labour

Placenta: 350 grams

Obduction: no defects

At birth, several congenital and/or chromosomal defects were diagnosed. More defects became apparent during follow-up. These cases are shown in table 4.8 and the consequences for the analysis are indicated. As stated in section 2.2, some congenital defects are associated with a lower genetic growth potential, rather than with IUGR. Such cases were, therefore, excluded from further analysis.

Besides the IUFD with multiple anomalies, the following infants were excluded from the analysis. Four infants were diagnosed with cardiac defects, two shortly after delivery, the other two at the age of three months. An infant with a large VSD which resulted in a neonatal death was described above. One infant had a spina bifida and a hydrocephalus. In one infant, trisomy 21 was diagnosed after delivery. During follow-up, one infant developed hydrocephaly, necessitating the implantation of a drain. The development of the cerebral aqueduct, corpus callosum, and cerebellum was found to be disturbed, but the cause remained unclear. In conclusion, 70 infants could be analysed for IUGR in the intervention group and 72 in the control group. 
Table 4.8

Congenital and/or chromosal defects

\begin{tabular}{|c|c|c|c|c|}
\hline \multirow[t]{2}{*}{ Diagnosis } & \multirow{2}{*}{$\begin{array}{l}\text { Intervention } \\
\text { group } \\
\text { case no. }\end{array}$} & \multirow{2}{*}{$\begin{array}{l}\text { Control } \\
\text { group } \\
\text { case no. }\end{array}$} & \multirow{2}{*}{$\begin{array}{l}\text { Doppler } \\
\text { result }\end{array}$} & Excluded from analysis of: \\
\hline & & & & Costs Growth Neuro \\
\hline
\end{tabular}

Neonatal diagnosis

multiple congenital abnormalities

trisomy 21

29

80

ventricular septal defect

coarctation of the aorta

spina bifida/hydrocephalus

$14 \quad 141$

17

141

Diagnosis during follow-up

ventricular septal defect

atrial/ventricular septal defect

hydrocephalus

122

encephalitis

104,126

$\begin{array}{lll}9 & \text { normal } & * \\ 7 & \text { raised } & * \\ 1 & \text { raised } & * \\ & \text { normal } & * \\ & \text { normal } & *\end{array}$

$\begin{array}{ll}* & * \\ * & * \\ * & * \\ * & * \\ * & *\end{array}$

Number excluded from the analysis 4

Number included in the analysis $\quad 70$

${ }^{1}$ excluded from neurological development analysis from the time of the insult at six months

* excluded from that part of the analysis

In an infant with an Erb's paralysis after breech extraction, this was taken into account at assessments of neurological development. Two infants experienced meningitis just before their neurological examination at the age of six months, resulting in permanent neurological damage. Clearly, a further evaluation of the possible effect of IUGR on their development became impossible. Data before that point in time were included in the analysis.

In conclusion, congenital defects were excluded from the analysis for reasons illustrated in figure 2.4. Growth and development could naturally only be followed-up in live infants. The number of cases found in the denominators of the calculations and tables in the remaining part of this chapter are shown in table 4.9.

\subsubsection{Costs}

Intrauterine exclusion of haemodynamic compromise in suspected IUGR using Doppler blood flow velocity measurements and the subsequent prevention of unnecessary hospitalisation during pregnancy, can theoretically reduce the hospitalisation rate in the intervention group without having a detrimental influence on neonatal outcome. According to the questionnaire (Nienhuis 1994, chapter 7), the hospitalisation rate in suspected IUGR is approximately $69 \%$. The prevalence of genetic smallness in SGA is 
Table 4.9

Overview of the size of the population

\begin{tabular}{llll}
\hline Group & After randomisation & Neonates without defects & Neonates followed up \\
\hline intervention & 74 & 70 & 69 \\
control & 76 & 72 & 70 \\
total number & 150 & 142 & 139 \\
\hline
\end{tabular}

$\%$ hospitalised

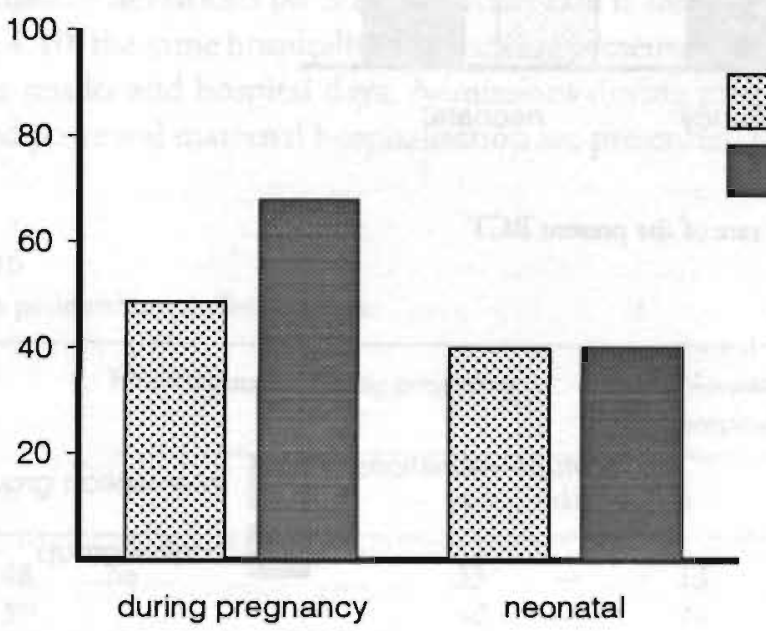

intervention group

control group

Figure 4.1

Expected hospitalisation rate in the control group according to the questionnaire and the expected reduced rate in the intervention group

Hospitalisation rate in the control group and neonatal hospitalisation rate were assessed by the questionnaire (chapter 7), $100 \%=$ total suspected IUGR in each group, during pregnancy = maternal hospitalisation rate during pregnancy; neonatal $=$ neonatal hospitalisation rate

not known from the literature, but is taken to be $30 \%$ (section 2.7.1). If hospitalisation in these cases is successfully prevented, the expected hospitalisation rates in the intervention and the control group are as shown in figure 4.1.

The actual hospitalisation rates are shown in figure 4.2. Contrary to expectations, the actual hospitalisation rate during pregnancy was higher in the intervention group than in the control group. The neonatal hospitalisation rate was similar in the two groups, as expected. 


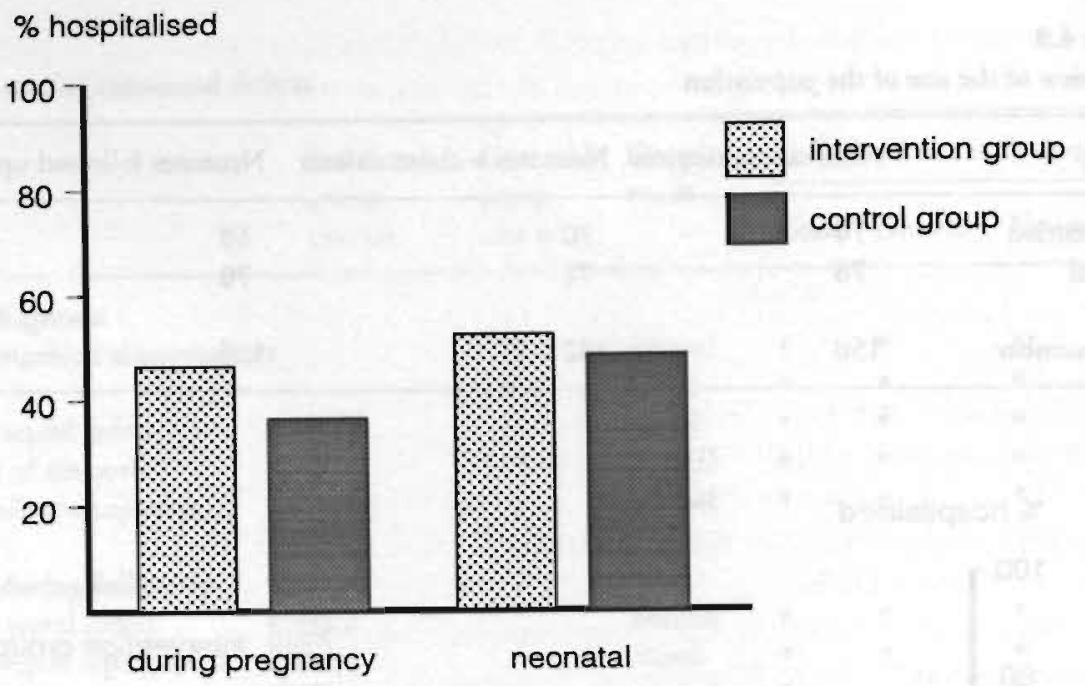

Figure 4.2

Actual hospitalisation rate of the present RCT

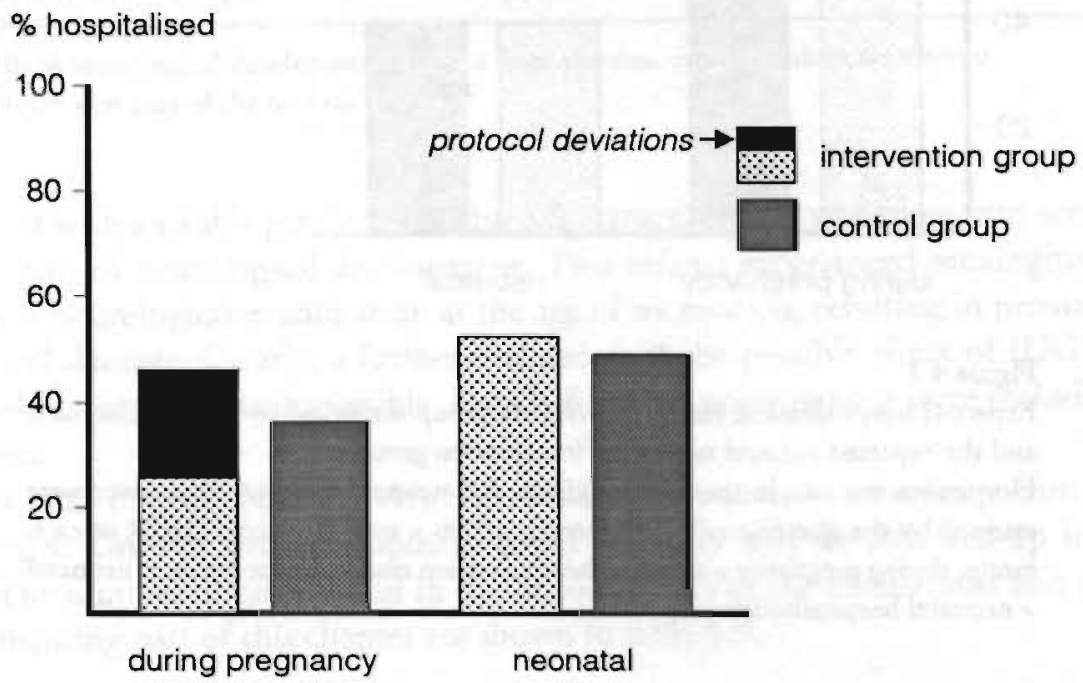

Figure 4.3

Actual hospitalisation rate of the present RCT and the protocol deviations 
According to the study hypothesis, the hospitalisation rate in the intervention group should have been reduced in comparison to the rate in the control group. Two aspects of this result are notable.

The estimated reduction in hospitalisation rate in the intervention group was achieved. The estimate in figure 4.1 is similar to the actual rate shown in figure 4.2. However, comparison of figure 4.2 and 4.1 shows that the hospitalisation rate during pregnancy in the control group is lower than expected from the questionnaire.

Secondly, closer examination of indications for admission to hospital of participants in the intervention group, reveals that 14 participants were admitted during pregnancy despite their normal Doppler results. These cases should not have been hospitalised for IUGR according to the study protocol. They are protocol deviations. The influence of these protocol deviations on hospitalisation rate is shown in figure 4.3.

In table 4.10, the same hospitalisation data are presented numerically with the associated Doppler results and hospital days. Admissions during pregnancy, neonatal hospitalisation, and postnatal maternal hospitalisation are presented. In the table, the results of the

Table 4.10

Complete perinatal hospitalisation data

\begin{tabular}{|c|c|c|c|c|c|c|c|c|}
\hline \multirow[t]{2}{*}{ Doppler } & \multirow[b]{2}{*}{$\mathbf{n}$} & \multicolumn{3}{|c|}{ Hospitalisation during pregnancy } & \multicolumn{2}{|c|}{$\begin{array}{l}\text { Neonatal } \\
\text { hospitalisation }\end{array}$} & \multicolumn{2}{|c|}{$\begin{array}{l}\text { Postnatal } \\
\text { hospitalisation }\end{array}$} \\
\hline & & & $\mathrm{n}$ & days & $\mathrm{n}$ & days & $\mathrm{n}$ & days \\
\hline \multirow[t]{6}{*}{ normal } & 48 & no & 33 & - & 13 & 127 & 13 & 68 \\
\hline & 50 & & 40 & - & 16 & 79 & 17 & 47 \\
\hline & & yes (protocol deviation) & 14 & 160 & 10 & 97 & 9 & 43 \\
\hline & & yes & 9 & 126 & 8 & 129 & 8 & 44 \\
\hline & & yes (not IUGR related) & 1 & 59 & 1 & 89 & 1 & 89 \\
\hline & & & 1 & 63 & 1 & 5 & 1 & 5 \\
\hline \multirow[t]{4}{*}{ raised } & 22 & no & 4 & - & 0 & 0 & 0 & 0 \\
\hline & 22 & & $5^{1}$ & - & 2 & 24 & $3^{1}$ & 14 \\
\hline & & yes & $18^{1}$ & 183 & 14 & 357 & $15^{1}$ & 80 \\
\hline & & & $17^{1}$ & 295 & 14 & 424 & 13 & 80 \\
\hline \multirow[t]{2}{*}{ total } & 70 & total hospitalisation: & $33 / 70$ & 402 & $38 / 69$ & 670 & $38 / 70$ & 280 \\
\hline & 72 & & $27 / 72$ & 484 & $41 / 70$ & 661 & $42 / 72$ & 190 \\
\hline
\end{tabular}

bold text: intervention group; italic text: control group

Doppler = 'worst' Doppler result; hospitalisation during pregnancy $=$ maternal hospitalisation during pregnancy; neonatal hospitalisation = admission to neonatal care unit, pediatric ward, or maternity ward; postnatal hospitalisation $=$ maternal hospitalisation after delivery; $\mathbf{n}=$ number of participants hospitalised; days = number of hospital days; protocol deviation = hospitalised during pregnancy despite normal Doppler results; ${ }^{1}$ includes one or more cases of intrauterine fetal death 
Table 4.11

Perinatal hospital days not related to IUGR

\begin{tabular}{lllll}
\hline Indication & $\begin{array}{l}\text { Case } \\
\text { no. }\end{array}$ & Doppler & $\begin{array}{l}\text { Hospital days } \\
\text { during pregnancy }\end{array}$ & $\begin{array}{l}\text { Neonatal } \\
\text { hospital days hospital days }\end{array}$
\end{tabular}

\begin{tabular}{lrlrrr}
\hline $\begin{array}{l}\text { Intervention group } \\
\text { psychosis }\end{array}$ & 103 & normal & 59 & 89 & 89 \\
donor kidney & 79 & normal & 0 & 2 & 2 \\
breech presentation & 1 & normal & 0 & 1 & 1 \\
naevus sebaceous & 30 & normal & 0 & 1 & 1 \\
total & & & 59 & 93 & 93 \\
Control group & & & & & \\
IDDM & 72 & normal & 63 & 5 & 5 \\
ulcerative colitis & 102 & raised & 0 & 3 & 3 \\
hyperthyroidism & 98 & normal & 0 & 7 & 7 \\
multiple sclerosis & 45 & normal & 0 & 1 & 1 \\
pregnancy induced DM & 16 & normal & 0 & 6 & 2 \\
breech presentation & 97 & normal & 0 & 2 & 2 \\
& 115 & normal & 0 & 1 & 1 \\
correction abdominal scar & 55 & normal & 0 & 2 & 2 \\
total & & & 0 & 3 & 3 \\
\hline
\end{tabular}

case no. $=$ participants registration number; IDDM/DM $=($ Insulin Dependent $)$ Diabetes Mellitus

intervention group are printed in bold and those of the control group in italics. In the first column, the participants are grouped according to their 'worst' Doppler result. This is the result which could have led to hospitalisation. The second column shows the hospitalisation during pregnancy and whether this was in accordance with the study protocol. The third column shows the neonatal hospitalisation. Note that the three intrauterine fetal deaths without congenital defects do not occur in the totals of this column. The fourth column is in parallel with the third and shows the associated postnatal maternal hospitalisation.

Not all hospital days were related to suspected IUGR, however, as can be expected in a high risk population. Table 4.11 shows the IUGR-unrelated indications, the participants registration number and group, the number of hospital days during pregnancy, the neonatal hospital days, and the maternal hospital days after delivery.

When these days are deducted from the data in table 4.10, only those hospital days which were related to suspected growth retardation remain for further analysis (table 4.12). Further analysis of cost will be restricted to these IUGR related hospitalisation data. 
Table 4.12

Perinatal hospitalisation data for suspected IUGR only

\begin{tabular}{|c|c|c|c|c|c|c|c|c|}
\hline \multicolumn{2}{|l|}{ Doppler } & \multicolumn{3}{|c|}{ Hospitalisation during pregnancy } & \multicolumn{2}{|c|}{$\begin{array}{l}\text { Neonatal } \\
\text { hospitalisation }\end{array}$} & \multicolumn{2}{|c|}{$\begin{array}{l}\text { Postnatal } \\
\text { hospitalised }\end{array}$} \\
\hline & $\mathrm{n}$ & & $\mathrm{n}$ & days & $\mathrm{n}$ & days & $\mathrm{n}$ & days \\
\hline \multirow[t]{4}{*}{ normal } & 48 & no & 34 & - & 12 & 124 & 11 & 65 \\
\hline & 50 & & 41 & - & 11 & 58 & 11 & 30 \\
\hline & & yes (protocol deviation) & 14 & 160 & 10 & 96 & 9 & 42 \\
\hline & & yes & 9 & 126 & 7 & 128 & 7 & 43 \\
\hline \multirow[t]{4}{*}{ raised } & 22 & no & 4 & - & 0 & 0 & 0 & 0 \\
\hline & 22 & & $5^{1}$ & - & 2 & 21 & $3^{1}$ & 11 \\
\hline & & yes & $18^{1}$ & 183 & 14 & 357 & $15^{1}$ & 80 \\
\hline & & & $17^{1}$ & 295 & 14 & 424 & 13 & 80 \\
\hline \multirow[t]{2}{*}{ total } & 70 & total hospitalisation: & $32 / 70$ & 343 & $36 / 69$ & 577 & $35 / 70$ & 187 \\
\hline & 72 & & $26 / 72$ & 421 & $34 / 70$ & 631 & $34 / 72$ & 164 \\
\hline
\end{tabular}

bold text: intervention group; italic text: control group

${ }^{1}$ includes one or more cases of intrauterine fetal death; (see legend table 4.10)

Of the live born infants, $52.2 \%(36 / 69)$ in the intervention group and $48.6 \%$ (34/70) in the control group needed in-hospital paediatric care. Neonatal hospital days were spent in the neonatal intensive care unit (NICU), the high care unit (NHCU), the medium care unit (NMCU), the paediatric ward (PW), and/or the maternity ward (MW) (table 4.13). These locations correspond to a decreasing intensity of medical and nursing care, severity of the fetal condition, and ultimately, to costs. Table 4.13 shows the distribution over the levels of care.

The statistical analysis of costs is presented in section 4.2.1.

\subsubsection{Neurological development}

The content of the Prechtl examination (Prechtl 1977) after delivery (or at term in preterm infants) and the neurological examinations according to Touwen (Touwen 1984) at the corrected ages of six and nine months is shown in appendix 1 and 2 . The methodology was described in section 3.3.3.

The average gestational age at the examinations did not differ significantly between the groups. If an examination could not be realised within the preset examination period, its content was adapted to include developmental items of the corrected age at the time of the examination.

The results of the neurological examinations after delivery and at the age of six and nine months are presented in table 4.14 and in figures 4.4 and 4.5. Appointments were missed 
Table 4.13

Neonatal hospitalisation

Level of care

Doppler Maternal hospitalisation Neonatal hospitalisation NICU NHCU NMCU PW MW during pregnancy

n total days

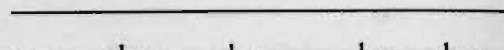

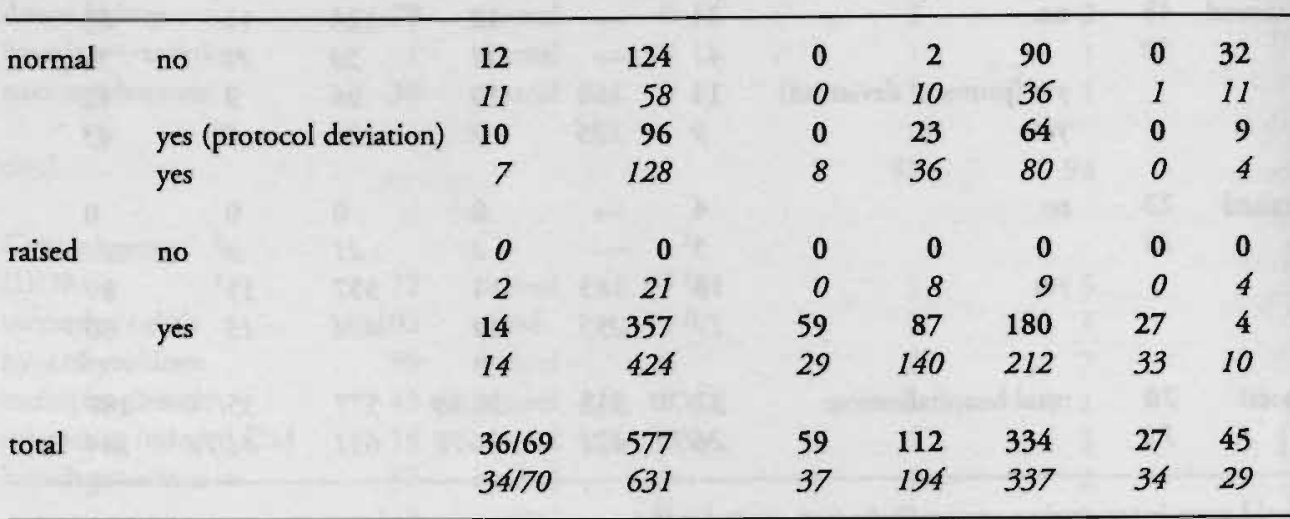

bold text: intervention group; italic text: control group

$\mathrm{NICU}=$ neonatal intensive care unit, $\mathrm{NHCU}=$ neonatal high care $\mathrm{unit}, \mathrm{NMCU}=$ neonatal medium care unit, $\mathrm{PW}=$ paediatric ward, $\mathrm{MW}=$ maternity ward

Table 4.14

Results of the neurological examinations

\begin{tabular}{|c|c|c|c|c|c|c|}
\hline \multirow{2}{*}{$\begin{array}{l}\text { Result } \\
\text { of } \\
\text { neurological } \\
\text { examinarions }\end{array}$} & \multicolumn{2}{|l|}{ After delivery } & \multicolumn{2}{|l|}{6 months } & \multicolumn{2}{|l|}{9 months } \\
\hline & $\begin{array}{l}\text { Intervention } \\
\text { group }\end{array}$ & $\begin{array}{l}\text { Control } \\
\text { group }\end{array}$ & $\begin{array}{l}\text { Intervention } \\
\text { group }\end{array}$ & $\begin{array}{l}\text { Control } \\
\text { group }\end{array}$ & $\begin{array}{l}\text { Intervention } \\
\text { group }\end{array}$ & $\begin{array}{l}\text { Control } \\
\text { group }\end{array}$ \\
\hline normal & 61 & 55 & 43 & 45 & 60 & 58 \\
\hline suspect & 5 & 8 & 20 & 20 & 6 & 9 \\
\hline abnormal & 0 & 1 & 0 & 0 & 0 & 1 \\
\hline missed & 3 & 6 & 4 & 5 & 1 & 2 \\
\hline $\begin{array}{l}\text { not included } \\
\text { in analysis }\end{array}$ & - & - & $2^{1}$ & - & $2^{1}$ & - \\
\hline total number & & & & & & \\
\hline of infants & 69 & 70 & 69 & 70 & 69 & 70 \\
\hline
\end{tabular}

${ }^{1}$ two cases of meningitis were not included in the analysis at six and nine months in the intervention group 
because of personal reasons, unrelated to the condition under investigation. Intrauterine fetal deaths are excluded from the denominators, as stated in table 4.9.

The results of an individual infant differ between examinations. The course of the results over the three neurological examinations, is presented in figures 4.4 and 4.5 for the intervention and the control group, respectively. The first column shows the number of infants and the results of the Prechtl examination after delivery (or at term in preterm infants) grouped according to diagnosis. In the second and third columns, the results obtained at six and nine months for each infant are shown. Because of the restricted number of 'abnormal' results, these were clustered with 'suspect' to 'not normal'.

Notable is that in both groups, a number of infants found to be normal at the Prechtl examination and at nine months, was found to be suspect at the corrected age of six months. Most of the infants regarded as suspect at the examination after delivery, were found to be normal at subsequent examinations.

The results of the neurological development follow-up are analysed further in section 4.2.3.

\subsubsection{Postnatal growth}

In the course of the study, the repeated anthropometric measurements proved to be too much of a burden to 24 of the participants. In the Netherlands, growth of all infants is followed-up by local child health centres, therefore, it was possible to use these data as a back-up.

Child health centre measurements of all participating infants were collected in order to establish the variation between AZM and child health centre anthropometrics. Data from neonates who had been measured at the AZM within one week after a visit to a child health centre were compared, retrospectively. Measurements at the AZM were in no way influenced by measurements made at the child health centres. In figures 4.6 to 4.8 , the differences between the paired measurements from the two locations are plotted against their averages (Campbell 1990). If the differences are small, the data points cluster about the line $y=0$. The average difference between the measurement locations is indicated by a line.

The average difference in height was $12 \mathrm{~mm}(\mathrm{n}=141$, median $=13 \mathrm{~mm}$, range $=-13-36$ $\mathrm{mm}), 0.4 \mathrm{~mm}$ in head circumference $(\mathrm{n}=181$, median $=0.5 \mathrm{~mm}$, range $=-19-17 \mathrm{~mm})$, and 107 grams in weight $(n=245$, median $=100$ grams, range $=-410-660$ grams). The differences in the number of eligible measurement pairs reflect the frequency with which such measurements are performed at the child health centres; weight often, and height less frequently. That the averages are greater than $y=0$ is in accordance with the fact that the median time interval between measurements at the child health centres and the AZM was five days. The small variation in head circumference measurements can be explained by the slower growth of the head and by the standardised measurement technique of the doctors at the child health centres. Height and weight are measured by trained aids. The greater systematic difference in height measurements reflects the 


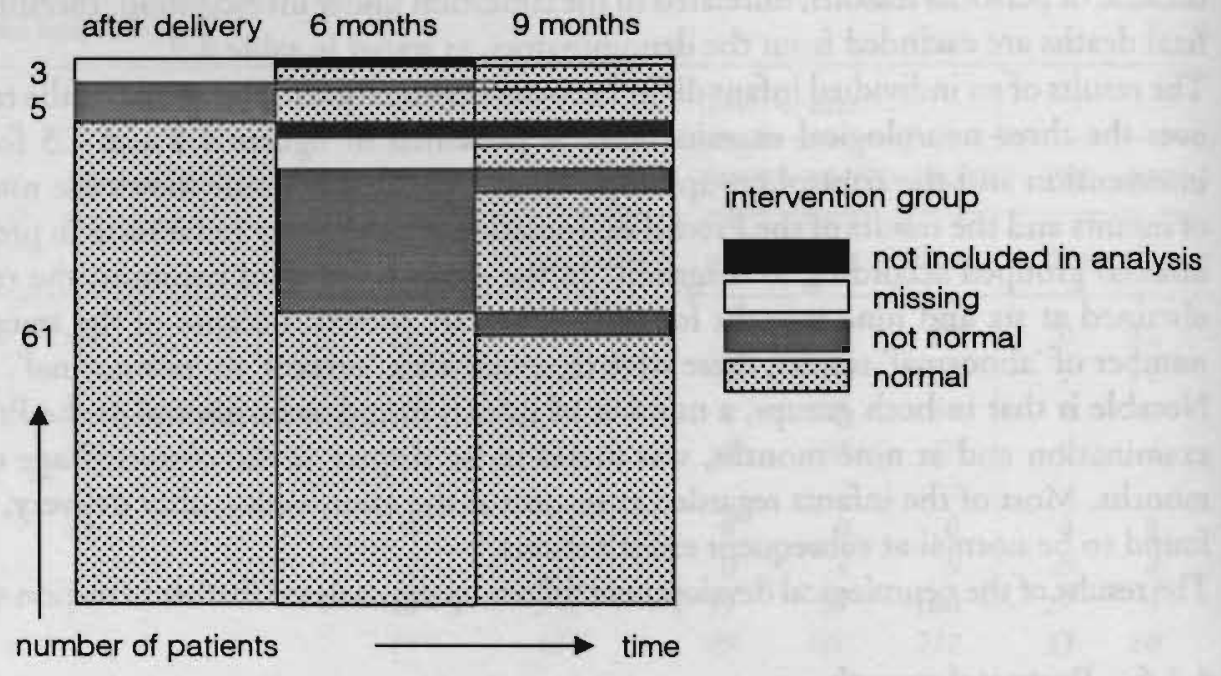

Figure 4.4

Intervention group: course of the individual neurological examination results Two infants with post encephalitis sequelae were not included in the analysis at six and nine months

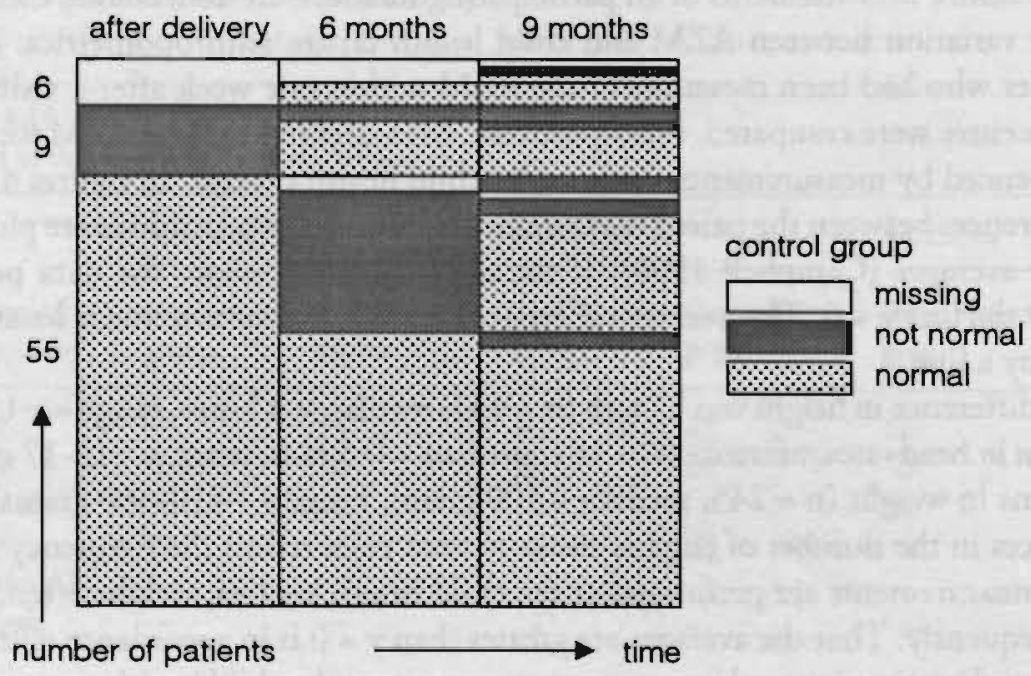

Figure 4.5

Control group: course of the individual neurological examination results 


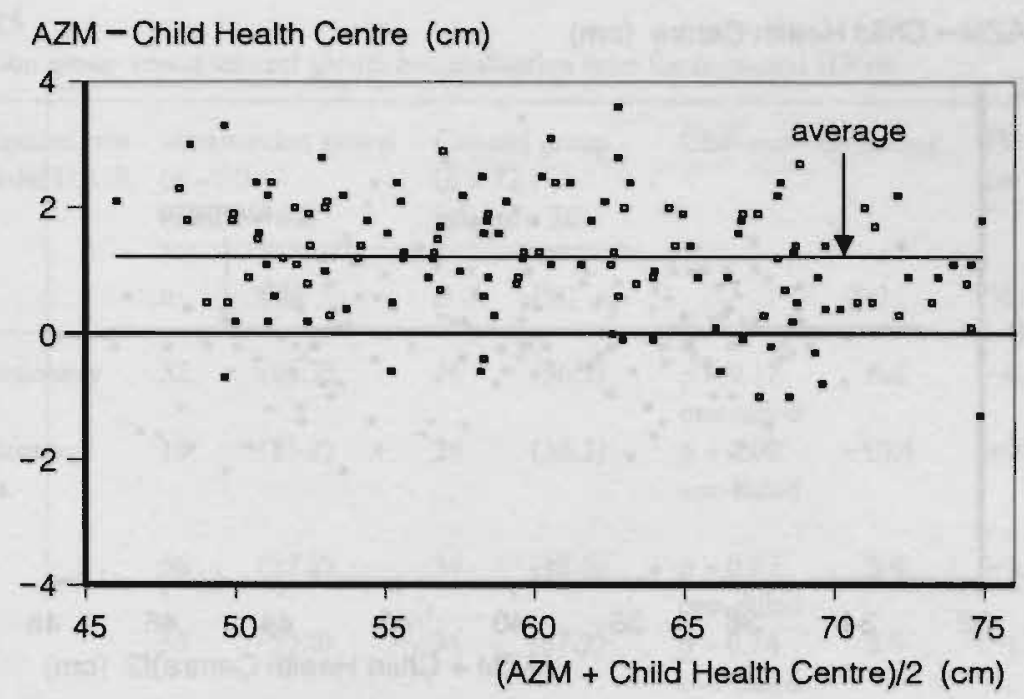

Figure 4.6

Height: AZM measurements versus Child Health Centre measurements

measurement protocol at the AZM which states that the infant should be firmly stretched.

The measurement differences found, were within reasonable limits. Moreover, if the difference in measurement result is systematic, for example due to a different standardisation of measurement technique at each location, the gain in size over a period of time will be similar.

Live born infants were measured an average of 10 times in the intervention group (range 3-16) and 10 times in the control group (range 4-14), including child health centre measurements for infants not or partly measured at the AZM. The standard deviation scores of all anthropometric measurements were calculated by using the formulas for the mean and standard deviations of the constructed reference curves (figures 3.8 to 3.10, appendix 3). Statistical analysis for differences in catch-up growth is performed in section 4.2.4.

\subsection{Analysis}

In the first part of this chapter, the data of the results were presented. In section 4.2, these data are analysed. The objective of the analysis was to test the dual hypothesis that the application of Doppler ultrasound measurements as reassurance test in suspected IUGR saves costs and is not detrimental to perinatal outcome. 


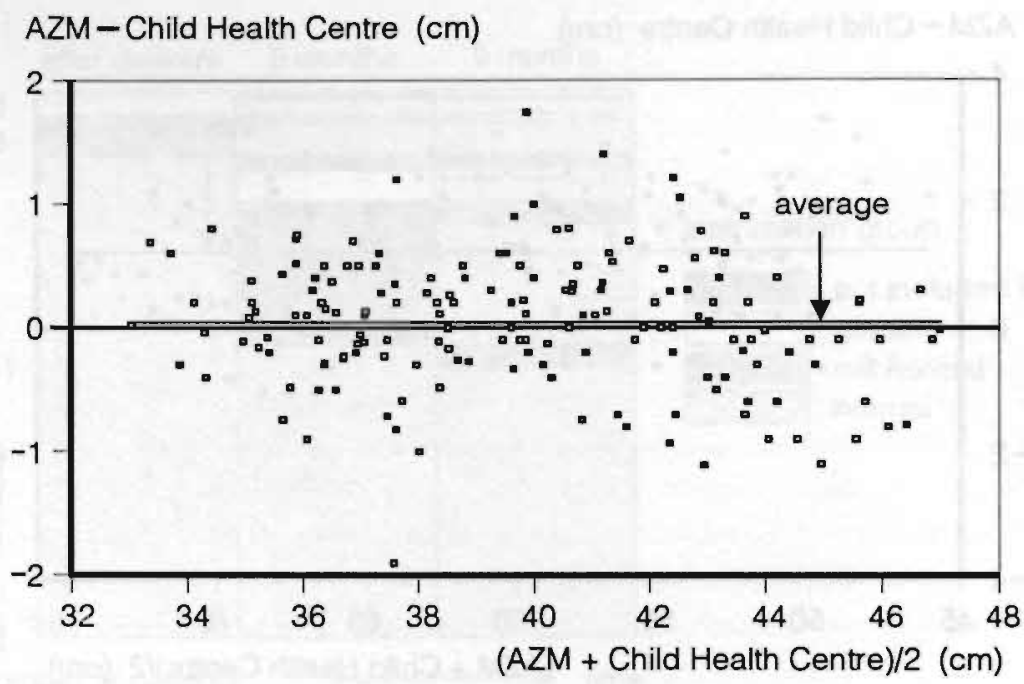

Figure 4.7

Head circumference: AZM measurements versus Child Health Centre measurements

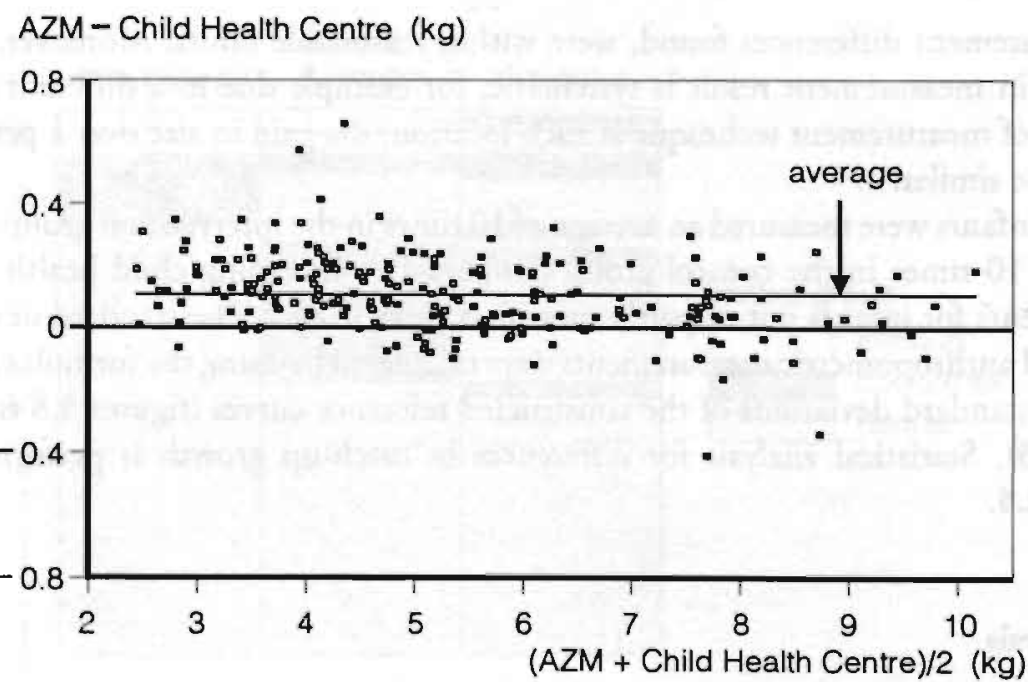

Figure 4.8

Weight: AZM measurements versus Child Health Centre measurements 
Table 4.15

Intervention group versus control group: hospitalisation rates for suspected IUGR

\begin{tabular}{|c|c|c|c|c|c|c|c|}
\hline \multirow[t]{2}{*}{$\begin{array}{l}\text { Hospitalisation rate } \\
\text { for suspected IUGR }\end{array}$} & \multicolumn{2}{|c|}{$\begin{array}{l}\text { Intervention group } \\
(n=70 / \\
\text { infants }=69)\end{array}$} & \multicolumn{2}{|c|}{$\begin{array}{l}\text { Control group } \\
(\mathrm{n}=72 / \\
\text { infants }=70)\end{array}$} & \multirow[t]{2}{*}{$\mathrm{Chi}^{2}$-test } & \multirow{2}{*}{$\begin{array}{c}\text { difference } \\
\text { (\%) }\end{array}$} & \multirow{2}{*}{$\begin{array}{l}95 \% \mathrm{Cl} \text { of } \\
\text { the difference } \\
\text { (\%) }\end{array}$} \\
\hline & $\mathrm{n}$ & $(\%)$ & $\mathrm{n}$ & $(\%)$ & & & \\
\hline during pregnancy & 32 & $(45.7)$ & 26 & $(36.1)$ & $\begin{array}{l}\mathrm{p}=0.12 \\
\text { one-tailed }\end{array}$ & 9.6 & $(-6.5-25.7)$ \\
\hline $\begin{array}{l}\text { without protocol } \\
\text { deviations }\end{array}$ & 18 & $(25.7)$ & 26 & $(36.1)$ & $\begin{array}{l}p=0.09 \\
\text { one-tailed }\end{array}$ & -10.4 & $(-25.5-4.7)$ \\
\hline neonatal & 36 & $(52.2)$ & 34 & $(48.6)$ & $\begin{array}{l}\mathrm{P}=0.67 \\
\text { two-tailed }\end{array}$ & 3.6 & $(-13.0-20.2)$ \\
\hline postnatal & 35 & $(50.0)$ & 34 & $(47.2)$ & $\begin{array}{l}\mathrm{p}=0.74 \\
\text { two-tailed }\end{array}$ & 2.8 & $(-13.6-19.2)$ \\
\hline
\end{tabular}

\subsubsection{Costs}

According to the hypothesis, the application of Doppler ultrasound measurements as a reassurance test in suspected IUGR would significantly reduce hospitalisation in the intervention group in comparison with the control group. In table 4.15, the hospitalisation rates from tables 4.12 and 4.13 are summarised, and this hypothesis is tested.

Contrary to expectations, more patients were admitted to hospital during pregnancy in the intervention group than in the control group. Table 4.15 shows that this difference was not statistically significant (one-tailed $\mathrm{Chi}^{2}$-test, $\mathrm{p}=0.12$ ). A one-tailed test was used as the direction of the result was specified in advance.

Clinical practice, however, does not always comply with the guidelines of a study protocol. If the study protocol had been strictly adhered to, fourteen participants in the intervention group with normal Doppler results would not have been hospitalised for IUGR. However, if these 14 protocol deviations had not been hospitalised, the difference in hospitalisation rate would not have been significant either (one-tailed $\mathrm{Chi}^{2}$ test, $\mathrm{p}=0.09$ ).

The indications for hospitalisation of the 14 protocol deviations were retrospectively and independently scrutinised by two gynaecologists. Suspected growth retardation was the sole reason for hospitalisation in half of the protocol deviations. These cases should therefore not have been admitted to hospital according to the trial's protocol.

In the remaining cases, additional indications besides the suspected IUGR influenced the decision to hospitalise. This included one case of suspected rupture of the membranes. Loss of amniotic fluid could not be demonstrated, however, and the patient was allowed to go home. She went into labour spontaneously at a later date and was 
Table 4.16

Intervention group versus control group: duration of hospitalisation for suspected IUGR

\begin{tabular}{|c|c|c|c|c|c|c|c|}
\hline \multirow[t]{2}{*}{$\begin{array}{l}\text { Hospital days for } \\
\text { suspected IUGR }\end{array}$} & \multicolumn{2}{|c|}{$\begin{array}{l}\text { Intervention group } \\
(n=70 / \\
\text { infants }=69)\end{array}$} & \multicolumn{2}{|c|}{$\begin{array}{l}\text { Control group } \\
(n=72 / \\
\text { infants }=70)\end{array}$} & \multicolumn{2}{|c|}{ t-test difference } & \multirow{2}{*}{$\begin{array}{l}95 \% \mathrm{CI} \text { of } \\
\text { the difference } \\
\text { (\%) }\end{array}$} \\
\hline & total & (average) & total & (average) & & $(\%)$ & \\
\hline during pregnancy & 343 & $(10.7)$ & 421 & $(16.2)$ & $\begin{array}{l}P=0.03 * \\
\text { one-tailed }\end{array}$ & -5.5 & $(-10.9--0.1)$ \\
\hline $\begin{array}{l}\text { without protocol } \\
\text { deviations }\end{array}$ & 183 & $(10.2)$ & 421 & $(16.2)$ & $\begin{array}{l}\mathrm{P}=0.02 * \\
\text { one-tailed }\end{array}$ & -6.0 & $(-11.5--5.4)$ \\
\hline neonatal & 577 & $(16.0)$ & 631 & $(18.6)$ & $p=0.59$ & -2.6 & $(-11.7-6.6)$ \\
\hline postnatal & 187 & $(5.3)$ & 164 & $(4.8)$ & $\begin{array}{l}\text { two-tailed } \\
p=0.68 \\
\text { two-tailed }\end{array}$ & 0.5 & $(-2.4-3.5)$ \\
\hline
\end{tabular}

* significantly different

Table 4.17

Intervention group versus control group: out-patient visits, ultrasound scans and CTG monitoring

\begin{tabular}{lrrrrr}
\hline Medical procedures & \multicolumn{2}{c}{ Intervention group } & \multicolumn{2}{l}{ Control group } \\
\cline { 2 - 3 } \cline { 5 - 6 } & total & (average) & total & (average) \\
\hline out-parient visits & 311 & $(4.4)$ & 282 & $(3.8)$ \\
ultrasound scans & 13 & $(0.2)$ & 12 & $(0.2)$ \\
CTG monitoring & 564 & $(8.0)$ & 558 & $(7.8)$ \\
\hline
\end{tabular}

admitted to the labour ward. In three cases, the obstetric history of a former intrauterine fetal death influenced admission. In individual cases, additional indications besides the suspected IUGR such as hypertension, decreased fetal movements, and abdominal pain were also of influence on admission.

In the opinion of the reviewers, the decision to hospitalise was understandable from the point of view of the clinician in charge in two cases: the case with the ruptured membranes and one case of suspected IUGR with a previous IUFD.

In conclusion, the uncompromising clinical management prescribed by the protocol does not take personal perceptions into account or factors in the individual's medical history, as a result of which a patient or her doctor fails to be reassured by a test result. Cases whereby the protocol of the trial is not adhered to, are by definition protocol 
deviations. However, in a number of these cases the non-adherence was clinically rational.

Although, the objective to reduce the number of hospitalisations during pregnancy for suspected IUGR in the intervention group in comparison to the control group, seems to have failed. However, comparison of these results (figure 4.2) with the expected results according to the hypothesis (figure 4.1) shows that, in the intervention group the estimated percentage of cases hospitalised during pregnancy for suspected IUGR was similar to the actual percentage. It was the actual hospitalisation rate in the control group which was lower than expected. These two effects, protocol deviations and reduction of hospitalisation in the control group, might have contributed to the not significant difference in hospitalisation rate.

The difference in duration of hospital admission between the intervention group and the control group, is shown in table 4.16. In general, duration of admission during pregnancy was significantly shorter in the intervention group than in the control group $(p=0.03)$. However, the $95 \% \mathrm{CI}$ of the difference between the groups was wide. If the protocol had been strictly adhered to, the statistically significant reduction in the duration of hospitalisation would have been strengthened by a less wide $95 \% \mathrm{CI}$ (table 4.16).

Average neonatal hospital days were not significantly different between the groups (two-tailed t-test: $\mathrm{p}=0.59$ ). Neither were postnatal days.

The study protocol could lead to changes in the number of medical procedures performed within the study period, other than hospital admission. Hospital admission data had been collected prospectively, but procedures such as out-patient visits, CTGs, and ultrasound scans, had not. These were tallied retrospectively from the AZM financial administration. Table 4.17 shows that the occurrence of medical procedures is comparable in the intervention and in the control group. However, when these data were checked using the patient's notes, they were not complete. This suggests that the registration system was not flawless. Although the data collected retrospectively from the patient's notes were not reliable either, these were also comparable between the groups. The outpatient procedures can, therefore, not be analysed. However, it is probable that flaws in a registration system occur at random. The similarity in the occurence of the procedures between the groups suggests that the protocol has not lead to significant differences between the groups. Therefore, it became irrelevant to perform a detailed tally of less costly procedures, such as laboratory procedures, or to assess the true costs for the above procedures.

\subsubsection{Perinatal outcome}

If haemodynamic insufficiency can be excluded by Doppler ultrasound measurements in fetuses suspected of IUGR, continued outpatient management of these pregnancies instead of in-hospital management, will not be to the detriment of the fetuses concerned. The object of the statistical analysis was to assess whether this hypothesis was justified. 
Table 4.18

Intervention group versus control group: perinatal outcome

\begin{tabular}{|c|c|c|c|c|c|c|c|}
\hline \multirow[t]{2}{*}{ Outcome } & \multicolumn{2}{|c|}{$\begin{array}{l}\text { Intervention group } \\
(\mathrm{n}=70)\end{array}$} & \multicolumn{2}{|c|}{$\begin{array}{l}\text { Control group } \\
(n=72)\end{array}$} & \multirow[t]{2}{*}{$\begin{array}{l}\text { two-tailed } \\
\mathrm{Chi}^{2} \text {-test }\end{array}$} & \multirow{2}{*}{$\begin{array}{l}\text { difference } \\
(\%)\end{array}$} & \multirow{2}{*}{$\begin{array}{l}95 \% \mathrm{CI} \text { of } \\
\text { the difference } \\
(\%)\end{array}$} \\
\hline & $\mathrm{n}$ & $(\%)$ & $\mathrm{n}$ & $(\%)$ & & & \\
\hline \multicolumn{8}{|l|}{ induction of labour } \\
\hline for IUGR & 15 & $(21.4)$ & 15 & $(20.8)$ & $\mathrm{p}=0.93$ & 0.6 & $(-22.8-14.0)$ \\
\hline elective CS & 6 & $(8.6)$ & 4 & $(5.6)$ & $\mathrm{p}=0.48$ & 3.0 & $(-5.4-11.4)$ \\
\hline emergency CS & 2 & (2.9) & 7 & $(9.7)$ & $\mathrm{p}=0.09$ & -6.8 & $(-14.7-1.0)$ \\
\hline preterm birth & 13 & $(18.6)$ & 12 & $(16.7)$ & $\mathrm{p}=0.77$ & 1.9 & $(-10.6-14.4)$ \\
\hline birth weight $\leq 2500 \mathrm{~g}$ & 29 & $(41.4)$ & 22 & $(30.6)$ & $\mathrm{p}=0.18$ & 10.8 & $(-4.8-26.6)$ \\
\hline birth centile $<\mathrm{P} 10$ & 33 & $(47.1)$ & 41 & $(56.9)$ & $p=0.24$ & -9.8 & $(-26.2-6.6)$ \\
\hline
\end{tabular}

\section{Table 4.19}

Intervention group versus control group: neurological development

\begin{tabular}{|c|c|c|c|c|c|c|c|}
\hline \multirow{2}{*}{$\begin{array}{l}\text { Neurological } \\
\text { development }\end{array}$} & \multicolumn{2}{|c|}{$\begin{array}{l}\text { Intervention group } \\
\text { (infants }=69 \text { ) }\end{array}$} & \multicolumn{2}{|c|}{$\begin{array}{l}\text { Control group } \\
\text { (infants }=70)\end{array}$} & \multirow[t]{2}{*}{$\begin{array}{l}\text { two-tailed } \\
\text { Chi }^{2} \text {-test }\end{array}$} & \multirow{2}{*}{$\begin{array}{l}\text { difference } \\
(\%)\end{array}$} & \multirow{2}{*}{$\begin{array}{l}95 \% \mathrm{CI} \text { of } \\
\text { the difference } \\
(\%)\end{array}$} \\
\hline & $\mathbf{n}$ & $(\%)$ & $\mathbf{n}$ & $(\%)$ & & & \\
\hline 'after delivery' & $5 / 66$ & $(7.6)$ & $9 / 64$ & (14.1) & $p=0.23$ & -6.5 & $(-17.7-4.7)$ \\
\hline six months & $20 / 63$ & $(31.7)$ & $20 / 65$ & $(30.8)$ & $\mathrm{p}=0.91$ & 0.9 & $(-15.1-17.0)$ \\
\hline nine months & $6 / 66$ & $(9.1)$ & $10 / 68$ & (14.7) & $p=0.32$ & -5.6 & $(-16.5-5.3)$ \\
\hline
\end{tabular}

\section{Table 4.20}

Intervention group versus control group: catch-up growth in the first six months after delivery

\begin{tabular}{|c|c|c|c|c|c|c|c|}
\hline \multirow[t]{2}{*}{ Carch-up growth } & \multicolumn{2}{|c|}{$\begin{array}{l}\text { Intervention group } \\
\text { (infants }=69 \text { ) }\end{array}$} & \multicolumn{2}{|c|}{$\begin{array}{l}\text { Control group } \\
\text { (infants }=70 \text { ) }\end{array}$} & \multirow[t]{2}{*}{$\begin{array}{l}\text { two-tailed } \\
\text { Chi }{ }^{2} \text { test }\end{array}$} & \multirow{2}{*}{$\begin{array}{l}\text { difference } \\
(\%)\end{array}$} & \multirow{2}{*}{$\begin{array}{l}95 \% \mathrm{CI} \text { of } \\
\text { the difference } \\
\text { (\%) }\end{array}$} \\
\hline & $\mathrm{n}$ & $(\%)$ & $\mathrm{n}$ & $(\%)$ & & & \\
\hline weight & 23 & (33.3) & 34 & $(48$ & $\mathrm{P}=$ & -15.3 & $(-3$ \\
\hline height & 15 & $(21.7)$ & 25 & $(35.7)$ & $p=0.07$ & -14.0 & $(-28.8-0.9)$ \\
\hline head circumference & 21 & $(30.4)$ & 24 & (34.3) & $p=0.63$ & -3.9 & $(-19.4-11.7)$ \\
\hline
\end{tabular}


In the literature, a number of parameters is traditionally used to assess perinatal outcome (for instance those used in the meta-analysis of the RCTs, tables 2.7-2.12). None of these parameters can be regarded as a gold standard for intrauterine growth retardation. Later in this chapter, another parameter is proposed as a standard for IUGR. In this section, the difference in obstetric management and neonatal outcome between the intervention and the control group was tested, using the traditional parameters (table 4.18).

The table shows that the intervention group and the control group have a comparable outcome. The application of the Doppler based management in the intervention group does not evidently lead to an increase in perinatal risk. For comparison, the prevalence of these outcomes in singleton pregnancies at the AZM obstetric outpatient clinic in 1989 is shown in table 3.1 .

\subsubsection{Neurological development}

The results of the neurological follow-up were presented in section 4.1.5. If the Doppler based intervention only withholds in-hospital treatment in low risk pregnancies, this management policy will not be detrimental to infants in the intervention group. This is the second part of the dual study hypothesis. In the analysis shown in table 4.19, the hypothesis is tested by comparing the occurrence of not normal neurological assessments after delivery, at six, and at nine months in the intervention group with the occurence in the control group.

The results of the neurological examinations shown in table 4.19 do not differ significantly between the two study groups at any of the three examinations. In conclusion, the Doppler based intervention does not seem detrimental to postnatal neurological development.

\subsubsection{Postnatal (catch-up) growth}

On the basis of the literature presented in section 2.7.1, postnatal catch-up growth was suggested as a retrospective standard for IUGR. Catch-up growth was defined as an upward change in anthropometric parameters of $\geq 1$ SDS in the first six months after delivery. This time span allows adequate time for preterm infants to overcome initial postnatal problems, while limiting the influence of the frequency and nature of the nutrition, both of which vary increasingly after the age of six months.

The summary of the postnatal growth process by two measurement points is only adequate if the individual infant's anthropometric values progress smoothly without outliers or sharp bends. Therefore, the repeated measurements of weight, height, and $\mathrm{HC}$ of each infant were plotted against age. On visual scrutiny, all the resulting individual growth curves were seen to follow a smooth course.

Standard deviation scores were calculated as described in section 3.3.4. The difference in SDS between the anthropometric measurements after delivery and the measurements 
closest to the sixth month after delivery were used to establish the presence of catch-up growth. The results are shown in table 4.20.

The occurrence of catch-up growth in weight, did not differ significantly between the two groups. Neither did the occurrence of catch-up in height or head circumference. The confidence intervals of the difference between the groups show a trend towards a lesser occurrence of catch-up in the intervention group, but as previously stated, this is not significantly so. In conclusion, if IUGR is retrospectively defined by the occurrence of catch-up growth, no detrimental effect of the study protocol on this aspect of outcome could be found.

\subsection{Summary of the results}

According to the literature, normal Doppler ultrasound measurements are associated with a low perinatal risk, regardless of fetal size. Normal Doppler results in suspected growth retardation should guarantee normal perinatal outcome, regardless of the application of optimal clinical treatment or overtreatment. Therefore, the dual hypothesis tested in the analysis of the randomised clinical trial presented in this thesis was that the application of Doppler ultrasound measurements as a reassurance test in suspected intrauterine growth retardation:

1. reduces costs in the intervention group in comparison to the control group

2. is not detrimental to outcome

The main analysis shows that the intention to reduce costs by reducing hospitalisation in suspected IUGR was not fully realised in this trial. In fact, the hospitalisation rate for suspected intrauterine growth retardation in the intervention group was not significantly different from that in the control group. The duration of hospitalisation during pregnancy, however, differed significantly between the two groups $(p=0.03)$. If no protocol deviations had occurred, a reduction in the mean duration of hospitalisation of 5 to 11 days would have been achieved in the intervention group compared to the control group.

Other medical procedures which contribute to costs, such as outpatient visits, ultrasound scans, and CTG monitoring during the study period, occurred in a similar rate in both groups, although such data should be collected prospectively.

There was no significant difference between the two groups in neurological development. Postnatal catch-up growth occurred in a similar rate in both groups.

A secondary analysis is performed in chapter 5 to elucidate the relation between the Doppler ultrasound results and the outcome parameters of the trial.

The findings in this and the following chapter will be discussed in chapter 6 . 


\section{Chapter 5}

\section{Secondary analysis: Doppler ultrasound results versus outcome of the randomised clinical trial}

In chapter 4, the results of the RCT were presented and the differences between the intervention group and the control group in perinatal outcome, costs, neurological development, and postnatal growth were analysed.

In this chapter, the relations between these outcome parameters and the Doppler ultrasound results are presented in a secondary analysis. This analysis is reported in a separate chapter to distinguish these secondary results from the main objective of the trial. Such a secondary analysis can provide further insight into the validity of Doppler ultrasound measurements in the umbilical artery in suspected intrauterine growth retardation and lead to hypotheses for further study.

\subsection{Doppler ultrasound results versus costs}

The relation between hospitalisation rate during pregnancy and Doppler ultrasound results is displayed in figure 5.1. Hospitalisation rate was notably lower if the Doppler ultrasound results were in the normal range. Similarly, if the Doppler results were raised, $80 \%$ of the patients was hospitalised during pregnancy. This effect was comparable in the intervention and the control group.

These results indicate that high fetal risk is adequately identified during routine prenatal care. It is conceivable that participation of a patient in the study improved the validity of prenatal outpatient check-ups by increasing the alertness of the clinicians in charge. Alternatively, the risk estimation provided by Doppler ultrasound measurements seems comparable to that of routine prenatal care. If there is doubt about the risks involved, as in suspected IUGR, non-invasive, relatively easy to perform Doppler ultrasound assessment may therefore be of use in clinical decision making.

\subsection{Doppler ultrasound results versus perinatal outcome}

The relation between the Doppler ultrasound results and perinatal outcome is explored in figures 5.2 and 5.3. Figure 5.2 shows the chances per randomisation group of an 


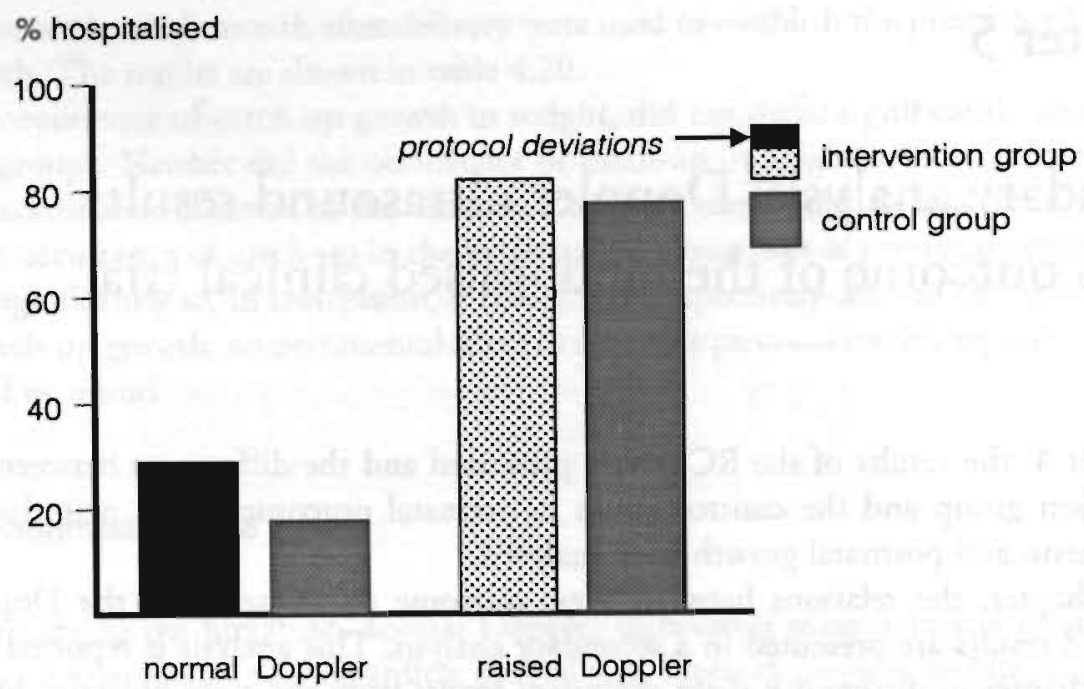

Figure 5.1

Actual hospitalisation rate during pregnancy and Doppler ultrasound results

uneventful outcome in cases with normal Doppler results throughout the study period (intervention group $n=48$, control group $n=50$ ). Figure 5.3 depicts the chance of an uneventful outcome in cases where one or more raised Doppler results could have lead to hospitalisation (intervention group $\mathrm{n}=22$, control group $\mathrm{n}=22$ ).

In the intervention group, 14 participants were hospitalised for IUGR despite their normal Doppler ultrasound results. To clarify whether these cases should be regarded as high or low risk, obstetric intervention and perinatal outcome of the protocol deviations were compared to other subgroups with normal Doppler ultrasound results (table 5.1). There were four normal Doppler subgroups: the protocol deviations, the hospitalised subgroup in the control group, and the two not hospitalised subgroups in the intervention and the control group.

Comparing the four normal Doppler subgroups, it is notable that the proportion of low birth weight $(50 \%)$ and SGA infants $(50 \%)$ among the protocol deviations is more comparable with that of the hospitalised subgroup in the control group, than with the not hospitalised subgroups. That these fetuses were regarded as high risk is evident from the high percentage of inductions of labour, which is comparable to that of the hospitalised control subgroup, and the even higher percentage of elective CSs.

However, the proportion of emergency CSs and preterm births, which gives an indication of perinatal risk, is far lower in the protocol deviations than in the hospitalised subgroup in the control group, and more comparable to the not hospitalised subgroups. Although a high proportion of neonates in the protocol deviations is hospitalised, again comparable with the hospitalised control subgroup, the duration of hospitalisation is more than a week shorter. Finally, the occurrence of catch-up growth and not normal 


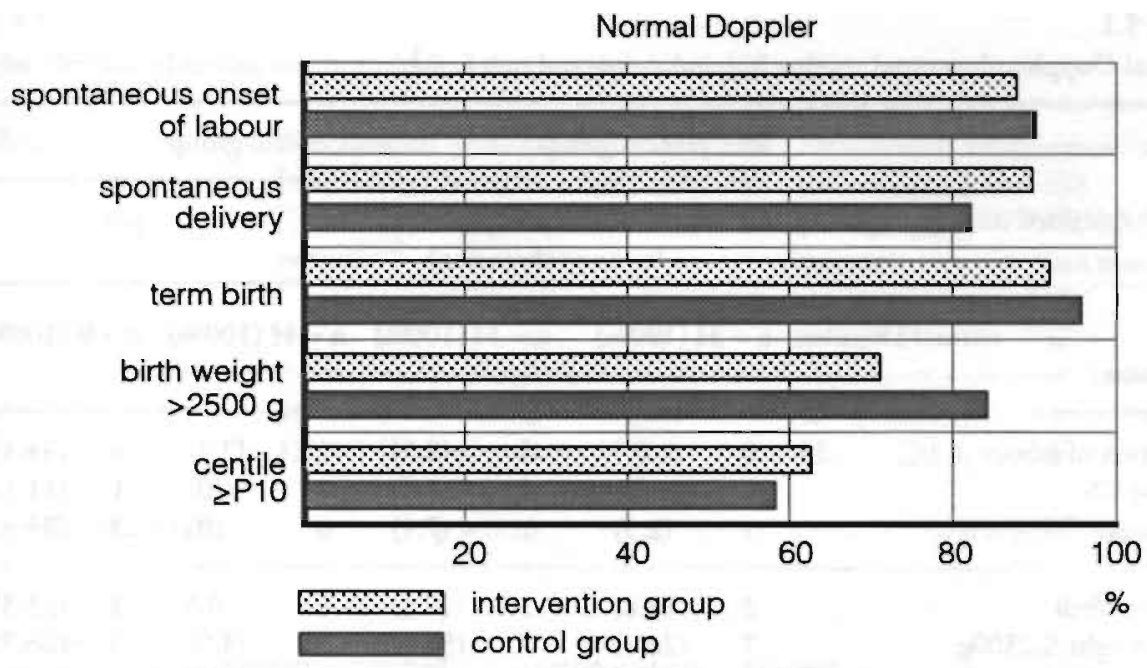

Figure 5.2

Probability of an uneventful outcome if the Doppler results are normal

Raised Doppler

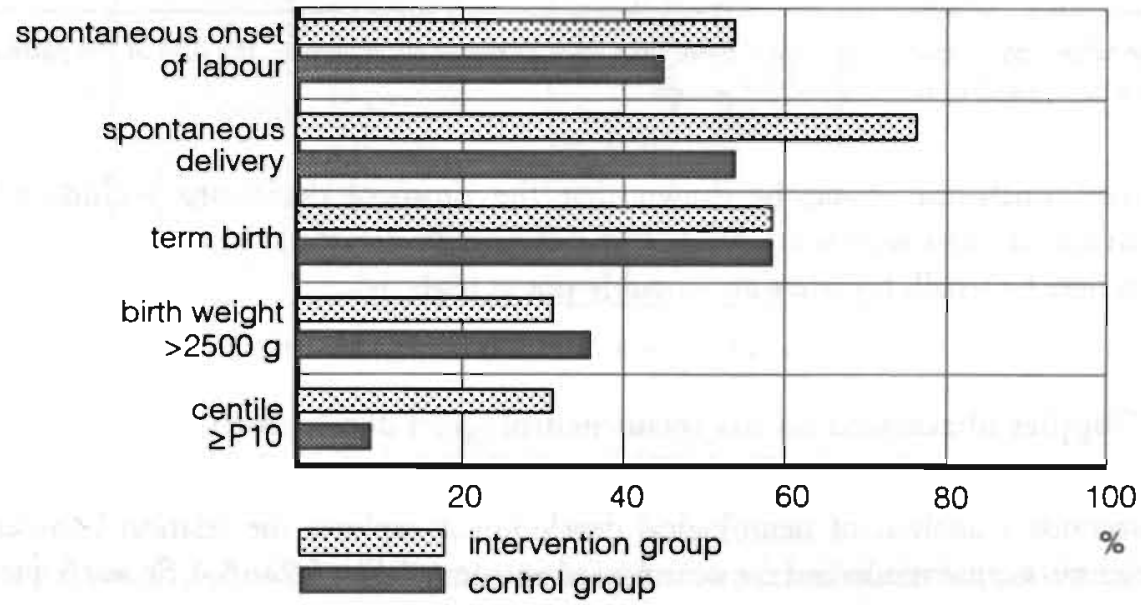

Figure 5.3

Probability of an uneventful outcome if the Doppler results are raised

neurological development at nine months is more comparable to that of the not hospitalised subgroups in both study groups.

Breaking down the study population into subgroups leads to very small numbers per group. Comparisons between subgroups can therefore be misleading. However, the 
Table 5.1

Normal Doppler ultrasound results, hospitalisation and outcome ${ }^{1}$

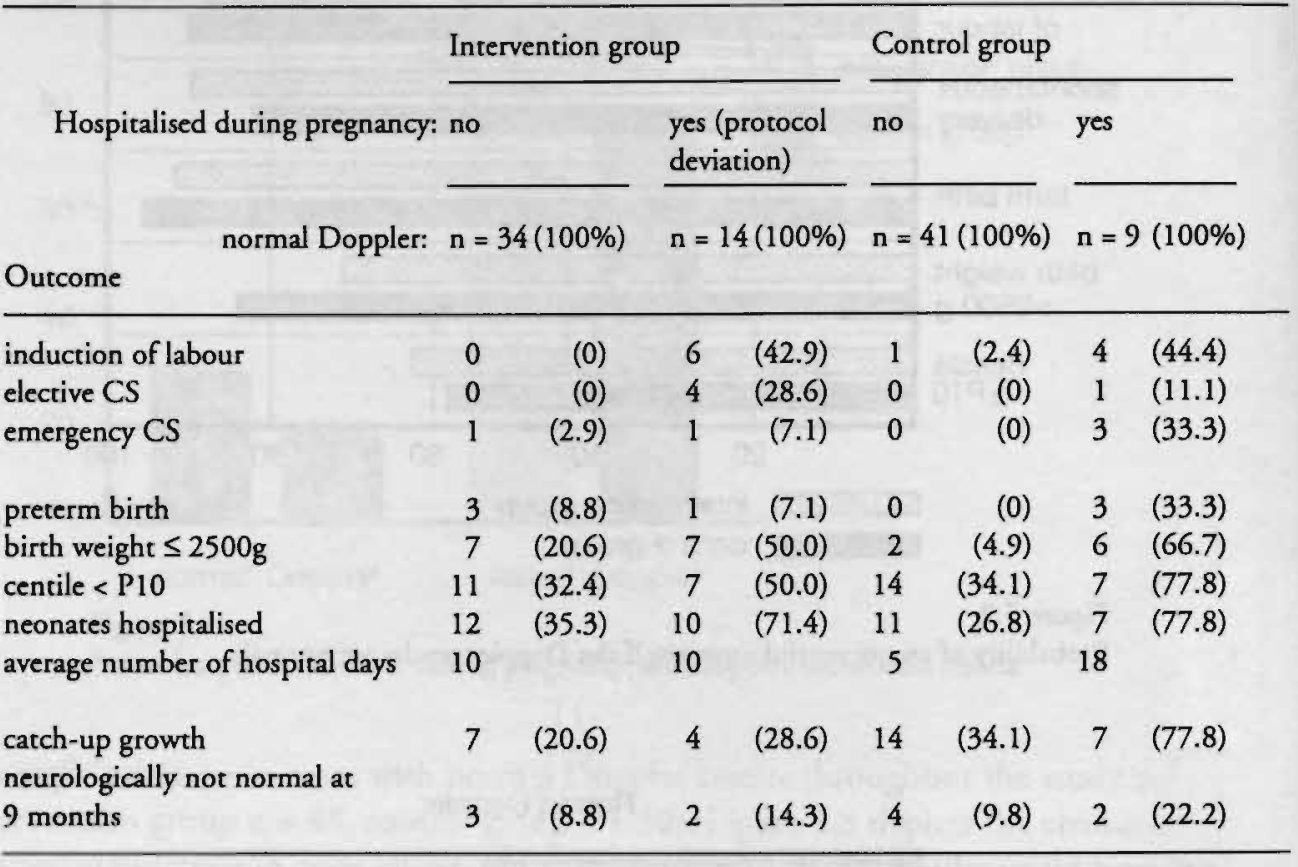

\footnotetext{
${ }^{1}$ in the table a participant may appear more than once or not at all, therefore, the sum of the participants cannot be equated to the totals of the groups
}

tentative conclusion could be drawn that the protocol deviations include a high proportion of cases which are SGA, but not growth retarded. In other words, these fetuses may be small, but they are possibly not at high risk.

\subsection{Doppler ultrasound results versus neurological development}

The secondary analysis of neurological development explores the relation between the Doppler ultrasound results and the neurological outcome (tables 5.2 to 5.4 , figures 5.4 to 5.6). Table 5.2 and figure 5.4 show the chance per study group and per Doppler subgroup of being not normal at the neurological examination 'after delivery' (section 3.3.3). This chance tended to increase if the Doppler ultrasound results were raised.

At the Prechtl examination 'after delivery', the relation between normal Doppler ultrasound results and normal neurological development, and raised Doppler results and not normal neurological development, was significant for both study groups combined $\left(C h i^{2}\right.$-test, $\left.\mathrm{p}=0.00\right)$. This significant difference was only corroborated in the control group, however (table 5.2). 


\section{Table 5.2}

Doppler ultrasound results and neurological development 'after delivery'

\begin{tabular}{|c|c|c|c|c|c|c|c|c|}
\hline \multirow{3}{*}{ Doppler } & \multicolumn{4}{|c|}{$\begin{array}{l}\text { Neurological development in } \\
\text { Intervention group }(n=66)\end{array}$} & \multicolumn{4}{|c|}{$\begin{array}{l}\text { Neurological development in } \\
\text { Control group }(n=64)\end{array}$} \\
\hline & \multicolumn{2}{|c|}{ normal } & \multicolumn{2}{|c|}{ not normal } & \multicolumn{2}{|c|}{ normal } & \multicolumn{2}{|c|}{ not normal } \\
\hline & $\mathbf{n}$ & $(\%)$ & $\mathrm{n}$ & $(\%)$ & $\mathbf{n}$ & $(\%)$ & $\mathrm{n}$ & $(\%)$ \\
\hline normal & 44 & $(66.7)$ & 2 & $(3.0)$ & 42 & $(65.6)$ & 3 & $(4.7)$ \\
\hline raised & 17 & $(25.8)$ & 3 & $(4.5)$ & 13 & (20.3) & 6 & (9.4) \\
\hline $\mathrm{Chi}^{2}$-test for $2 \times 2$ table & & & & 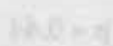 & & & & \\
\hline
\end{tabular}

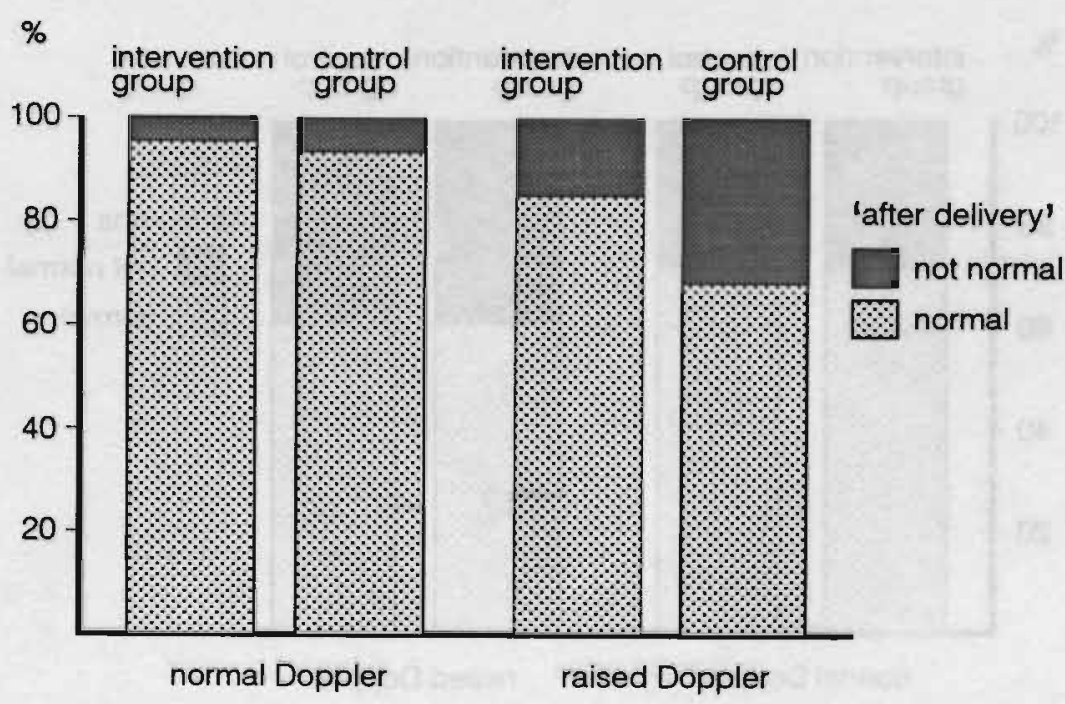

Figure 5.4

Doppler ultrasound results and neurological development 'after delivery'

Similarly, table 5.3 and figure 5.5 show the relation between Doppler ultrasound results and findings of the neurological examination at a corrected age of six months. The relation between the two parameters was weak.

Finally, the same comparison was made at the corrected age of nine months. Again there is no clear relation (table 5.4, figure 5.6).

There was a significant relation between the Doppler ultrasound results and the neurological development assessment 'after delivery' in both study groups combined. The association was found in the control group but not in the intervention group. An explanation could be that the intervention group had the benefit of Doppler information 
Table 5.3

Doppler ultrasound results and neurological development at six months

\begin{tabular}{|c|c|c|c|c|c|c|c|c|}
\hline \multirow{3}{*}{ Doppler } & \multicolumn{4}{|c|}{$\begin{array}{l}\text { Neurological development in } \\
\text { intervention group }(n=63)\end{array}$} & \multicolumn{4}{|c|}{$\begin{array}{l}\text { Neurological development in } \\
\text { control group }(n=65)\end{array}$} \\
\hline & \multicolumn{2}{|c|}{ normal } & \multicolumn{2}{|c|}{ not normal } & \multicolumn{2}{|c|}{ normal } & \multicolumn{2}{|c|}{ not normal } \\
\hline & n & (\%) & $\mathrm{n}$ & (\%) & $\mathrm{n}$ & (\%) & $\mathrm{n}$ & $(\%)$ \\
\hline normal & 32 & $(50.8)$ & 13 & $(20.6)$ & 34 & $(52.3)$ & 13 & $(20.0)$ \\
\hline raised & 11 & (17.5) & 7 & (11.1) & 11 & (16.9) & 7 & (10.8) \\
\hline $\mathrm{Chi}^{2}$-test for $2 \times 2$ table & \multicolumn{4}{|c|}{$p=0.44$} & \multicolumn{4}{|c|}{$p=0.38$} \\
\hline
\end{tabular}

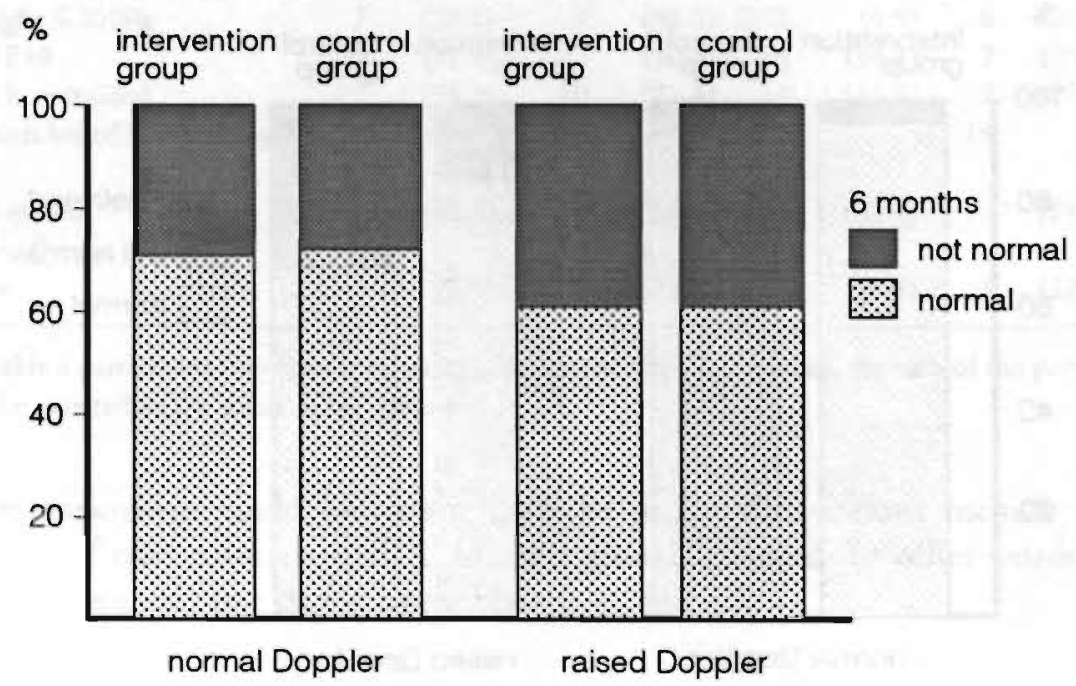

Figure 5.5

Doppler ultrasound results and neurological development at six months

in clinical decision making, which could have improved outcome. An association between Doppler ultrasonography and neurological outcome was not found at the corrected ages of six and nine months.

However, the neonates who were 'suspect' at the Prechtl examination had significantly lower gestational ages at birth and lower birth weights. Such an association between GA and birth weight, and neurological development was not found at the corrected ages of six and nine months. Even though the Doppler ultrasound results were not known, the infants appearance is an aspect to which the investigator cannot be blinded. In the days after delivery, the appearance of the neonate is most likely to bias the investigation. This 


\section{Table 5.4}

Doppler ultrasound resules and neurological development at nine months

\begin{tabular}{|c|c|c|c|c|c|c|c|c|}
\hline \multirow[t]{3}{*}{ Doppler } & \multicolumn{4}{|c|}{$\begin{array}{l}\text { Ncurological development in } \\
\text { intervention group }(n=66)\end{array}$} & \multicolumn{4}{|c|}{$\begin{array}{l}\text { Neurological development in } \\
\text { control group }(n=68)\end{array}$} \\
\hline & \multicolumn{2}{|c|}{ normal } & \multicolumn{2}{|c|}{ not normal } & \multicolumn{2}{|c|}{ normal } & \multicolumn{2}{|c|}{ not nurmal } \\
\hline & $\mathrm{n}$ & $(\%)$ & $n$ & $(\%)$ & $n$ & $(\%)$ & $\mathrm{n}$ & $(0,0)$ \\
\hline normal & 41 & $(62.1)$ & 5 & $(7.6)$ & 42 & $(61.8)$ & 6 & $(8.8)$ \\
\hline raised & 19 & $(28.8)$ & 1 & $(1.5)$ & 16 & $(23.5)$ & 4 & $(5.9)$ \\
\hline Chi²-test for $2 \times 2$ table & \multicolumn{4}{|c|}{$p=0.45$} & \multicolumn{4}{|c|}{$p-0.43$} \\
\hline
\end{tabular}

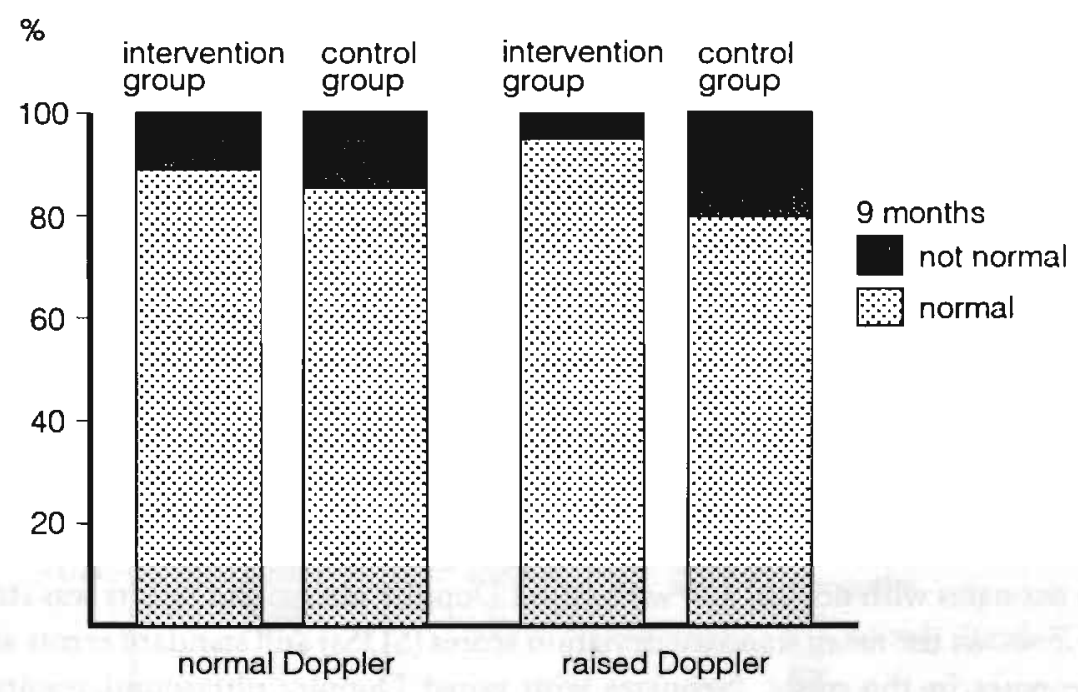

Figure 5.6

Doppler ultrasound results and neurological development at nine months

is a probable explanation for the significant finding. Finally, the significant finding could also be due to chance (type I error).

\subsection{Doppler ultrasound results versus postnatal growth}

If IUGR is retrospectively defined by the occurrence of catch-up growth after delivery, the prevalence of genetic smallness can be calculated. The presence of the condition of interest, which is 'no catch-up growth', is set out in a two by two table (table 5.5) against 
Table 5.5

The detection of genetic smallness by Doppler ultrasound measurements

\begin{tabular}{|c|c|c|c|}
\hline Doppler result & $\begin{array}{l}\text { No catch-up } \\
\text { n }\end{array}$ & $\begin{array}{l}\text { Carch-up } \\
\mathbf{n}\end{array}$ & total number \\
\hline normal & 66 (a) & 32 (b) & 98 \\
\hline raised & 16 (c) & 25 (d) & 41 \\
\hline total number & 82 & 57 & 139 \\
\hline
\end{tabular}

(see figure 2.5)

the outcome of the test, as was previously shown in figure 2.5. In this case 'normal Doppler values' form the positive test. From this table, the prevalence of genetic smallness among live born infants in the trial was calculated to be $59 \%$. The sensitivity with which Doppler ultrasound measurements in the umbilical artery predict genetic smallness was $80 \%$.

In this secondary analysis, the relation between the Doppler assessments of intrauterine haemodynamic sufficiency, and the anthropometric parameters after delivery and at the chronological age of six months was analysed further. For this secondary analysis, the whole study population was divided into two Doppler subgroups: a normal and a raised Doppler subgroup. The literature in section 2.4 reviewed the changes in the different anthropometric parameters in IUGR. A deficient intrauterine environment would effect weight most, followed by length. The head circumference was found to be relatively spared in comparison to weight and length.

The difference at birth in the deviation of anthropometric parameters from the mean between neonates with normal and with raised Doppler ultrasound results was striking. Figure 5.7 shows the mean standard deviation scores (SDSs) and standard errors at birth of all neonates in the study. Neonates with raised Doppler ultrasound results were significantly lighter $(\mathrm{p}=0.00)$ and shorter (marginally significant $\mathrm{p}=0.046)$ than their normal Doppler peers. Their head circumferences did not differ significantly $(p=0.94)$, which supports the concept of the 'brain sparing effect'.

Six months after delivery (figure 5.8), the difference in weight between the Doppler result subgroups is no longer significant $(p=0.14)$. Height at six months remains marginally shorter in infants with raised Doppler values in comparison to infants with normal Doppler values $(p=0.049)$.

If figures 5.7 and 5.8 are compared, the deviation in all parameters from the mean is shown to be less negative at six months than after delivery. This shows that the majority of the infants have a tendency to catch-up to a certain degree. The upward change in SDSs of weight towards the mean is significantly greater in infants with raised Doppler 


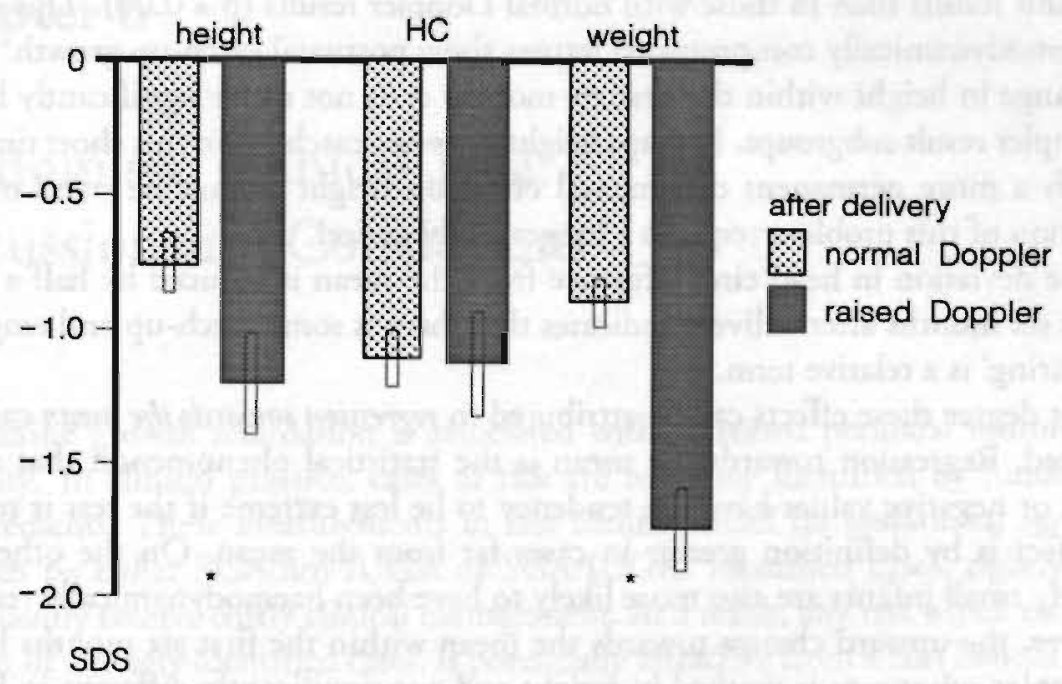

\section{Figure 5.7}

Doppler ultrasound results and anthropometric parameters at birth

Results are shown as mean and standard error; * difference between the normal and the raised Doppler subgroup is significant

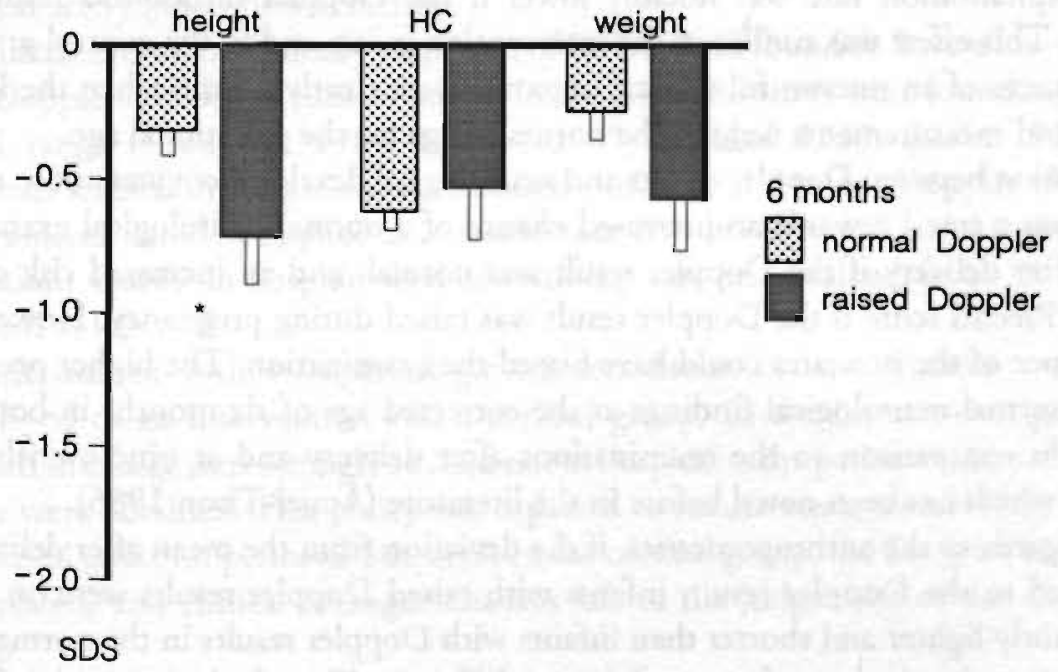

\section{Figure 5.8}

Doppler ultrasound results and anthropometric parameters at six months Results are shown as mean and standard error; ${ }^{*}$ difference berween the normal and the raised Doppler subgroup is significant 
ultrasound results than in those with normal Doppler results $(p=0.00)$. This suggests that haemodynamically compromised fetuses show postnatal catch-up growth.

The change in height within the first six months does not differ significantly between the Doppler result subgroups. Perhaps height does not catch-up in this short time span, although a more permanent detrimental effect on height cannot be ruled out. The elucidation of this problem requires a longer study period.

That the deviation in head circumference from the mean is reduced by half a SDS in the first six months after delivery indicates that there is some catch-up and emphasises that 'sparing' is a relative term.

To what degree these effects can be attributed to regression towards the mean cannot be quantified. Regression towards the mean is the statistical phenomenon that extreme positive or negative values have the tendency to be less extreme if the test is repeated. This effect is by definition greater in cases far from the mean. On the other hand, extremely small infants are also more likely to have been haemodynamically restricted. Moreover, the upward change towards the mean within the first six months between the Doppler subgroups is marked in weight and not significantly different in $\mathrm{HC}$ and length. This corresponds to a physiologically conceivable pattern of asymmetrical growth retardation, which was discussed previously in section 2.4 .

\subsection{Summary of the results}

The hospitalisation rate was notably lower if the Doppler ultrasound results were normal. This effect was similar in the intervention group and in the control group.

The chances of an uneventful clinical outcome were clearly greater when the Doppler ultrasound measurements were in the normal range for the gestational age.

The relation between Doppler results and neurological development was not so marked. There was a trend towards an increased chance of a normal neurological examination result after delivery if the Doppler result was normal, and an increased risk of a not normal Prechtl score if the Doppler result was raised during pregnancy. However, the appearance of the neonates could have biased the examination. The higher proportion of not normal neurological findings at the corrected age of six months in both study groups in comparison to the examinations after delivery and at nine months was a finding which has been noted before in the literature (Amiel-Tison 1986).

With regards to the anthropometrics, if the deviation from the mean after delivery was compared to the Doppler result, infants with raised Doppler results were on average significantly lighter and shorter than infants with Doppler results in the normal range. The average head circumference did not differ significantly between the Doppler subgroups. At the age of six months, the deviation from the mean had decreased notably in weight, height, and head circumference. The difference in weight was no longer significant between the two Doppler subgroups. The difference in height remained marginally significant. 


\section{Chapter 6}

\section{Randomised Clinical Trial: Discussion and Conclusions}

Intrauterine growth retardation is associated with increased perinatal morbidity and mortality. In clinical practice, cases at risk are routinely identified by fundal height measurements. These measurements in fact identify small for gestational age fetuses, who can be either SGA/not-IUGR or SGA/IUGR. Identified cases, rightly or not, subsequently receive costly clinical management. As a result, any test which reduces the number of wrongly identified cases, is potentially attractive from a cost benefit point of view.

In a proportion of SGA fetuses, smallness is genetically determined. A pathophysiological mechanism underlying SGA/IUGR is utero-placental (haemodynamic) insufficiency. Therefore, a test which assesses the haemodynamic link between mother and fetus might limit the number of false positive classifications of fundal height measurements. The eligible test for this purpose is Doppler ultrasonography of the umbilical artery blood flow velocities.

In the literature, cases clinically suspected of IUGR were found to have a low perinatal risk if Doppler ultrasound measurements in the umbilical artery were found to be in the normal range (Trudinger 1985, Reuwer 1987, Haddad 1988, Burke 1990). The hypothesis studied in the present randomised clinical trial was that Doppler ultrasound measurements could be applied as a 'reassurance test' in suspected intrauterine growth retardation. Costly in-hospital fetal monitoring can be refrained from in the cases identified as low risk.

Pregnant women with a suspected growth retardation of two or more weeks wcre randomised to an intervention and a control group. In the intervention group, the clinicians in charge were strongly advised not to hospitalise the patient if normal Doppler profiles were obtained. This policy was expected to reduce costs, without this having a detrimental effect on perinatal outcome. In the control group, the Doppler results were not revealed, and clinical management was left to the judgement of the clinician in charge.

The results which were presented in full in chapter 4, are summarised in table 6.1 and are subsequently discussed below. 
Table 6.1

Summary of expected versus obtained results between the intervention group and the control group

Expected

Reduction of the prenatal maternal hospitalisation rate and duration for suspected IUGR in the intervention group, in comparison to the control group
Obtained

- The hospitalisation rate in the intervention group does not differ significantly from that in the control group

- The hospitalisation rate in the intervention group is reduced, as expected

- The hospitalisation rate in the control group is lower than expected from the survey in Dutch university hospitals

- The duration of hospitalisation is significantly shorter in the intervention group than in the control group

No significant differences between the intervention and control groups in:

- perinatal outcome

- postnatal catch-up growth

- neurological development

\subsection{Costs}

The maternal hospitalisation rate during pregnancy was not decreased in the intervention group in comparison to the control group. However, almost half the admissions in the intervention group had normal Doppler ultrasound results and should not have been admitted for IUGR according to the study protocol. The effect of protocol deviations may have been less if the study protocol had been enforced upon the clinicians in charge. In the planning of the trial, the choice between enforcing the non-hospitalisation policy in case of a normal Doppler, and strongly advising this intervention was discussed with the epidemiologist A.R. Feinstein (Yale University School of Medicine, New Haven, USA). If the intervention is enforced, the maximal effect of the intervention is obtained (efficacy). The question arises whether this maximal effect is also optimal. Such ideal study results cannot be extrapolated to future clinical practice, because under less strict conditions the policy will not reach the same effectiveness (Belizán 1990). Moreover, there is a risk that the study ends up testing the power of the department's authority or that resistance against this power leads to unpredictable changes in the clinical management. If the intervention is strongly advised by the study protocol, it may be disregarded, which leads to protocol deviations. The results are those which can be obtained in clinical practice (effectiveness).

In this effectiveness study, the investigators chose to rely on the compliance of the participating clinicians, taking the risk of protocol deviations. An impression of the efficacy if the protocol had been strictly adhered to, can be obtained in an effectiveness 
study by analysing the results both with and without the protocol deviations. Therefore, both these results were presented in chapter 4 .

The hospitalisation rate was not reduced in the intervention group in comparison to the control group. This could lead to the simple conclusion that the trial has failed or that its basic assumptions were wrong. However, there are several objections against this conclusion. Firstly, in the intervention group, the clinicians in charge hospitalised a number of patients disregarding their normal Doppler results. Secondly, the hospitalisation rate in the intervention group actually coincided with the reduced hypothetical rate estimated before the start of the study. Thirdly, in the control group, which was managed according to the conventional clinical policy, the hospitalisation rate was lower than the expected $69 \%$ estimated by a questionnaire among $80 \%$ of the obstetricians at the Dutch university hospitals (Nienhuis 1994, chapter 7).

Possibly the implementation of the study protocol led to a more critical attitude towards hospitalisation in suspected IUGR. The knowledge that one's performance is monitored by a trial could well increase the alertness of the clinician in charge. This is analogous to the 'Hawthorne effect' (Bouter 1988). This leads to the conclusion that millions of Dutch guilders can be saved by a more critical attitude alone, regardless of the application of Doppler ultrasound techniques.

The costs and savings of the Doppler protocol were recalculated in table 6.2, using the results of the present randomised clinical trial. The estimations made before the start of the trial (table 3.4) were reproduced to facilitate the comparison of the estimates and the results.

The estimations of the hospitalisation rate and duration in suspected IUGR were replaced by the results of the control group. In the control group, $36 \%$ of participants with suspected IUGR was hospitalised for an average of 16 days. The prevalence of genetic smallness in the whole study population was $59 \%$ and the sensitivity of Doppler ultrasound measurements in its detection was $80 \%$. The average number of Doppler examinations was 5.9 in the intervention group and 5.7 in the control group.

The annual net savings calculated on a national basis are almost 39 million Dutch guilders, which is more than double the estimate made before the start of the trial (table 6.2). Therefore, the implementation of Doppler ultrasound measurements as a reassurance test in suspected IUGR is attractive from a costs point of view.

The factors which were assessed beforehand to be of greatest influence on the calculation by a sensitivity analysis were the 'percentage of genetically small fetuses' and the 'average number of hospital days' (Ament 1995). The latter differed by a day only and therefore cannot explain the difference in costs. The percentage of genetically small fetuses had been arbitrarily set at $30 \%$, as the value of this item could not be found in the literature. Using the retrospective definition of catch-up growth, the prevalence of genetically small fetuses was $59 \%$, which was almost twice the estimate. Finally, the sensitivity with which genetic smallness was identified by Doppler ultrasound measurements was $30 \%$ higher than estimated. These two factors explain the higher savings and therefore the higher net savings obtained in table 6.2 . 
Table 6.2

Cost-analysis on a national basis using the results of the present RCT

\begin{tabular}{|c|c|c|c|}
\hline Factors & $\begin{array}{l}\text { Estimates } \\
\text { (table 3.4) }\end{array}$ & $\begin{array}{l}\text { Results of } \\
\text { the RCT }\end{array}$ & $\begin{array}{l}\text { Number } \\
\text { of cases }\end{array}$ \\
\hline singleton deliveries ${ }^{1}$ & $100 \%$ & $100 \%$ & 193,780 \\
\hline suspected IUGR & $12 \%$ & $12 \%$ & 23,254 \\
\hline hospitalised for sIUGR & $69 \%$ & $36 \%$ & 8,371 \\
\hline genetically small & $30 \%$ & $59 \%$ & 4,939 \\
\hline mean number of hospital days & 15 & 16 & \\
\hline costs hospital day & Df. 1,000 & Dfl. 1,000 & \\
\hline costs Doppler examination & Dfl. $\quad 180$ & Dfl. 180 & \\
\hline mean number of Doppler examinations & 5 & 5.8 & $\log 1$ \\
\hline sensitivity of Doppler examination & $50 \%$ & $80 \%$ & 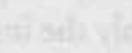 \\
\hline Savings & Dfl. $36,101,835$ & Dfl. $63,219,200$ & \\
\hline Costs & Dfl. $20,928,600$ & Dfl. $24,277,176$ & \\
\hline Net savings & Dfl. $15,173,235$ & Dfl. $38,942,024$ & \\
\hline
\end{tabular}

1 annual number of singleton births over 24 weeks gestational age in the Netherlands (CBS 1993) Dfl. $=$ Dutch guilders; Costs $=\left(\right.$ nbirths $\left.\times \%_{\text {sIUGR }}\right) \times($ costsDoppler $\times$ nDoppler $)$;

Savings $=\left(\mathrm{n}_{\text {births }} \times \%_{\text {sIUGR }} \times \%\right.$ genetically small $) \times(\%$ hospitalised $\times$ nhospital days $\times$ costshospital day $)$ $X$ sensitivity $;$ Net savings $=$ Savings - Costs

As the hospitalisation rate and the number of Doppler ultrasound examinations did not differ between the study groups, the true costs were not calculated. It must be kept in mind, however, that costs and savings may be influenced by the use of true costs in the calculation.

The cost-reducing effect of the Doppler based hospitalisation will also be influenced if private consequences of hospitalisation (loss of production, alternative home care, loss of leisure time) are taken into account. However, such costs are difficult to determine accurately. It is questionable whether their inclusion would change the trend suggested by the results.

A single Doppler ultrasound assessment would naturally be less costly than repeated measurements. In order to gain some insight into whether Doppler ultrasound assessment could have been limited to a single measurement at the onset of the suspicion of IUGR, the 'worst' Doppler result was compared to the first Doppler result in table 6.3. The Doppler findings in the umbilical artery deteriorated in nine cases in the intervention group (13\%) and in 11 cases in the control group (15\%). In almost half of these cases, the first Doppler results were in the indecisive range (orange). Two cases developed zero flow in the course of the study. These results indicate a progressively deteriorating haemodynamic situation which would not have been adequately assessed by a single Doppler ultrasound measurement. 
Table 6.3

Cases with a normal first Doppler result and a raised 'worst' Doppler result

\begin{tabular}{|c|c|c|c|c|c|}
\hline \multirow[t]{2}{*}{ Doppler result } & \multirow[b]{2}{*}{ first Doppler result } & \multicolumn{2}{|c|}{ Intervention group } & \multicolumn{2}{|c|}{ Control group } \\
\hline & & green & orange & green & orange \\
\hline \multicolumn{6}{|c|}{ 'worst' Doppler result } \\
\hline red & & 5 & 4 & 5 & 4 \\
\hline zero flow & & 0 & 0 & 1 & 1 \\
\hline
\end{tabular}

\subsection{Perinatal outcome}

Stratification and randomisation was performed to obtain comparable groups in most parameters. Stratification for more than the two prognostic parameters used (smoking and gestational age at randomisation) is not possible in a small trial such as this, as there are too few participants per stratum. Yet, the chance of an uneven distribution of a parameter is greater if the study population is small.

By chance some parameters were not equally distributed over the intervention group and the control group. The study groups differed significantly in the distribution of sex ( $58.1 \%$ boys and $36.8 \%$ boys, respectively), and breech presentations ( $4.1 \%$ and $18.4 \%$, respectively). Corrections were made by using sex specific growth references and by excluding hospital days specifically related to breech presentation in the analysis. The influence of such a skew distribution in secondary parameters is difficult to assess.

The perinatal outcome was comparable in the two study groups.

An extreme outcome parameter of any test in pregnancy is perinatal mortality. As the occurrence of perinatal mortality in industrialised countries is low, very large study populations are needed to prove a difference in perinatal mortality between the study groups. Moreover, not all perinatal mortality can be interpreted as an adverse outcome of a test. Perinatal deaths due to chromosomal and/or congenital defects are not a result of IUGR and can be associated with normal Doppler ultrasound results. In a case of severe IUGR in the intervention group, the parents decided after ample consideration to abstain from obstetric intervention. What the outcome would have been if intervention had taken place, cannot be assessed in retrospect. The effect of such cases in the analysis of IUFD is that the odds ratio of IUGR related perinatal death draws closer to ' 1 '. This indicates that the Doppler ultrasound measurements are not effective in the prevention of perinatal mortality. In the IUFD in the control group whereby the pregnancy was complicated by a donor kidney, IUFD may have been prevented if the Doppler results had been known.

In a large study population, skewed distributions and rare outcome parameters are less of a problem in the interpretation of the study results. 


\subsection{Neurological development}

In the occurrence of not normal neurological development, no significant differences were found between the intervention and the control group. This indicates that the Doppler ultrasound based policy has no detrimental effect on short term neurological development.

At the corrected age of six months, the proportion of infants with not normal neurological examination results was higher than after delivery or at nine months, in both the intervention group and in the control group. It was previously noted in the literature that transient abnormal neuromotor findings have a maximal expression between 6 and 8 months corrected age (Amiel-Tison 1986). In a study on the course of neurological findings in time, most abnormal findings resolved in the first nine months after delivery and normal development at the age of 5-7 years was found in $86 \%$ of these infants (Michaelis 1993). Such transitory abnormal neurological findings were thought to be the result of the relative immaturity of the neurological system at birth and were regarded, therefore, as expressions of normal neurobiological maturation of the central nervous system. Moreover, the neonatal brain has some capacity to recover from, or to compensate for perinatal insults. Besides, socio-economic factors are of great influence on long term development.

On the other hand, transient abnormal neurological findings in the first year after delivery are correlated with problems at school age (Nelson 1982, Amiel-Tison 1986). In the present trial, the focus was on the possible detrimental effects following limitation of overtreatment in low risk small infants. The duration of the neurological follow-up was limited by logistics. Therefore, short term neurological development was used as outcome measure in the present RCT. A follow-up of nine months was though relevant because, although some abnormal neurological findings may be transient, this does not detract from the fact that minor damage may have occurred. Moreover, if follow-up is performed at a later age, the influence of socio-economic factors and the occurrence of possibly damaging, childhood illnesses may confound the issue.

\subsection{Postnatal growth}

There was no significant difference between the intervention and the control group in the occurrence of catch-up growth. Again no negative effect of the Doppler ultrasound based policy was found.

If the occurrence of catch-up growth of $\geq 1 S D S$ in the first six months after delivery is used as a gold standard for IUGR, Doppler ultrasound measurements in the umbilical artery have a sensitivity of $80 \%$, a specificity of $44 \%$, and a likelihood ratio of a positive test of 1.4 and of a negative test of 0.4 . 
The secondary analysis showed that neonates with raised Doppler ultrasound results during pregnancy were significantly smaller and lighter at birth than their normal Doppler counterparts, but that their head circumferences did not differ significantly. These findings correlate well with the asymmetric proportions associated with IUGR in the literature and support the theory of a relative 'brain sparing effect'. This should more tentatively be called a 'head circumference sparing effect' as the functional consequences of this effect are not clear (Ounsted 1988).

The catch-up in weight in the first six months was significantly greater in cases with raised Doppler measurements than in cases with Doppler measurements in the normal range. The occurrence of catch-up growth may, therefore, retrospectively identify IUGR.

\subsection{Conclusions}

The main object of the randomised clinical trial described in this thesis was to test the dual hypothesis that the application of Doppler ultrasound measurements as a reassurance test in suspected IUGR:

1. leads to a less costly policy by decreasing unnecessary hospitalisation during pregnancy in low risk cases

2. has no detrimental influence on perinatal outcome

The expected difference in hospitalisation rate during pregnancy between the intervention group and the control group was not found. However, the implementation of the protocol did lead to an overall reduction in hospitalisation rate and number of hospital days in comparison with the rate estimated by gynaecologists in university hospitals. If calculated on a national scale, the savings could amount to almost 39 million Durch guilders.

No clear differences were found between the intervention and the control group in perinatal clinical outcome, neurological development and postnatal catch-up growth during the study period.

These results suggest that Doppler ultrasound measurements should be applied as reassurance test in clinical practice to support the risk assessment of fetuses suspected of intrauterine growth retardation. If the Doppler ultrasound findings are within the normal range, outpatient care is the management of choice in suspected IUGR. 


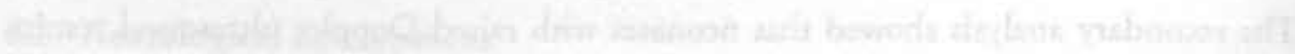

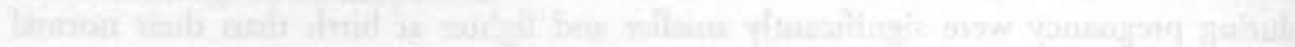

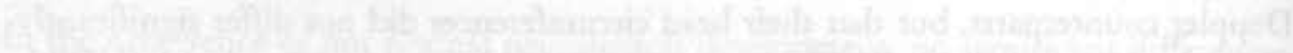

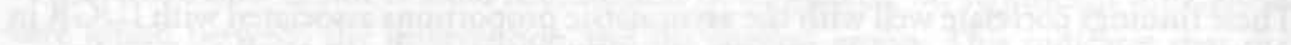

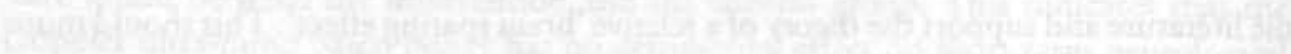

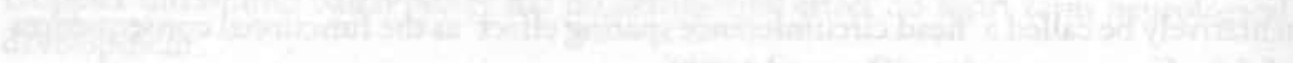

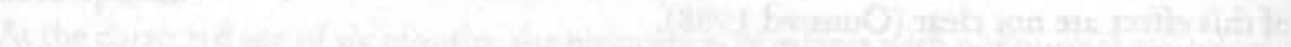

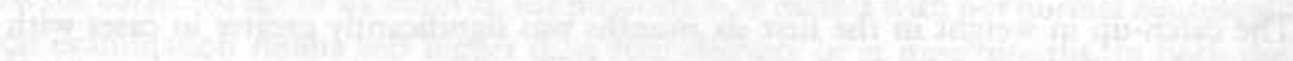

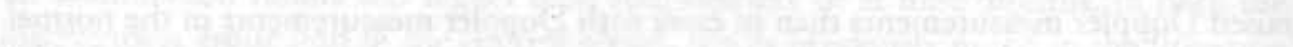

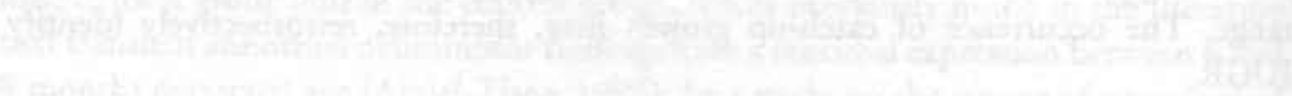

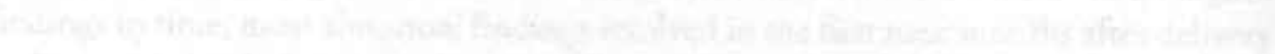

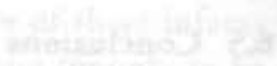

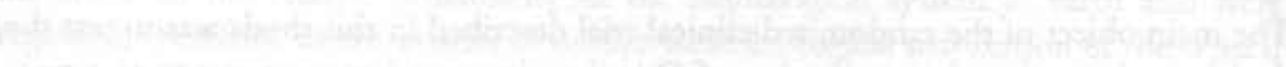
Bo7

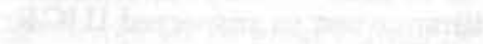

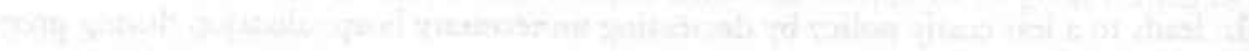




\section{Chapter $7^{1}$}

\section{Questionnaire: Routine clinical policy and application of Doppler ultrasound measurements in suspected intrauterine growth retardation in university hospitals in the Netherlands}

\subsection{Introduction}

A fetus or neonate is small for gestational age (SGA) if its weight is below a certain threshold value for the gestational age of the reference population. In the literature (Jones 1984, Altman 1989), several threshold values (2.3rd, 5th, or 10th centile) are used to define a fetus or neonate as being SGA. Intrauterine growth retardation (IUGR) is a condition in which the intrauterine growth curve bends down and away from its genetic growth potential due to a deficient materno-placental environment. It is clear that IUGR is not synonymous with SGA (Jones 1984, Wilcox 1983).

As intrauterine growth retardation is associated with increased perinatal morbidity and -mortality (Chiswick 1985, Heinonen 1985), a major goal of obstetric care is to diagnose IUGR. Tests used, either estimate fetal size (fundal height measurements, ultrasonic fetal biometry) or fetal wellbeing (cardiotocography (CTG), Doppler ultrasound measurements). Tests for fetal size, identify those who are SGA, but miss those who are growth retarded but whose size is not below the chosen threshold value, and falsely identify the genetically small/not-growth-retarded. Such false positive diagnosis, to concentrate on the latter, may subsequently lead to unnecessary, costly maternal hospitalisation and fetal monitoring.

Tests which assess fetal wellbeing rather than size, such as Doppler ultrasound measurements of the fetal circulation, are possibly more effective in distinguishing the genetically small SGA fetus from the growth retarded SGA fetus. Such tests could prove beneficial from a cost point of view, if unnecessary care in healthy, genetically small fetuses is prevented. In order to study the clinical effectiveness of such tests, they must be set off

\section{Based on:}

Nienhuis SJ, Zusterzeel NMAJ, Hoogland HJ. Routine clinical policy and application of Doppler measurements in suspected intrauterine growth retardation in university hospitals in the Netherlands. Eur J Obstet Gynecol Reprod Biol 1994; 56: 31-6. 
Table 7.1

Questionnaire

Definition and diagnosis of suspected IUGR

1. Which criterium for the duration of pregnancy do you use most often?

2. What threshold value (in fundal height or in fetal biometry) would you use to define IUGR?

3. At what gestational age is IUGR most commonly diagnosed?

4. In what percentage of cases with suspected IUGR are there further complications?

\section{Estimation of the magnitude of the problem of suspected IUGR}

5. How many cases of suspected IUGR do you personally diagnose a year?

6. What is your estimation of the number of cases with a growth retardation of two or more weeks at the outpatient clinic per year?

7. What percentage of cases with suspected IUGR do you personally decide to hospitalise?

8. What is your estimation of the total number of patients with suspected IUGR hospitalised annually at your hospital?

Clinical policy in suspected IUGR

9. In what percentage of patients do you check fetal size with ultrasonic biometry when you suspect IUGR clinically?

10. At what threshold value would you hospitalise a patient with suspected IUGR?

11. Under what circumstances would you discharge a patient with suspected IUGR from hospital?

12. Is there a consensus on clinical policy for suspected IUGR?

\section{Doppler ultrasound measurements}

13. Are Doppler measurements used in clinical decision making at your clinic?

14. Do you feel more/less certain about fetal wellbeing if a Doppler result is known for a patient with suspected IUGR?

15. If a patient with a clinically uneventful pregnancy has an abnormal Doppler result, what is your clinical policy?

16. If you could order Doppler ultrasound measurements routinely, would this change the frequency of CTG monitoring?

17. If you could order Doppler ultrasound measurements routinely, for which indications would you do so?

18. Do you find Doppler technologies an asset to antenatal diagnosis?

against the 'current clinical policy' on suspected IUGR in which the test is not applied. 'This can only be done, however, if there is such a thing as 'current clinical policy' and its content is known. Although the prevalence and clinical management of SGA is easily assessed, clinical intervention is applied to all cases in which intrauterine growth retardation is suspected, and such suspicions are not always recorded.

In the present study, routine clinical policy and the use of Doppler ultrasound techniques in suspected intrauterine growth retardation (sIUGR) were assessed by means of a questionnaire among obstetricians at the university hospitals in the Netherlands. 


\subsection{Methods}

The questionnaire was sent to all the obstetricians at the eight university hospitals in 1992. Non-respondents were reminded twice, either by mail or by telephone.

The questionnaire included twelve multiple choice and six 'open' questions. The former minimised the influence of chance, while the latter allowed for spontaneous information. The wording of the questions aimed to restrict each question to a single facet of the problem, limit its interpretation, and avoid leading questions (Bartelds 1989). Four questions addressed the definition and diagnosis of IUGR, four the local magnitude of the problem, four the clinical policy when growth retardation is suspected and six questions addressed the opinion of the clinicians on Doppler ultrasound measurements and the application of Doppler ultrasound in clinical practice. In order to put these answers into perspective, information on the prevalence of singleton pregnancy at the outpatient clinic and the availability of Doppler equipment at each university clinic was obtained.

The so called test-retest method was used to estimate the reliability of the questionnaire. Before the start of the study, the questionnaire was put to obstetricians at the University Hospital of Maastricht twice, with a time interval of one year. Numerical answers differed less than $5 \%$, and the order and relative magnitude of categorical answers were similar. The validity of the test was checked by assessing the cohesion between the answers to different questions probing the same problem. The questionnaire is summarised in table 7.1 and is available from the authors.

Statistical analysis was performed with the SPSS-PC+ statistical programme. Answers to mutiple choice questions are expressed as proportion and percentages of responding obstetricians.

\subsection{Results}

\subsubsection{Response}

The participating centres were: the university hospitals of Groningen, Leiden, Nijmegen, Maastricht, Rotterdam, Utrecht, the Free University of Amsterdam, and the University Medical Centre in Amsterdam. The results per hospital are presented in a random order.

The departments of obstetrics of all eight university hospitals agreed to participare in the study. The mean response in all university hospitals was $80.8 \%$ (range $=50-100 \%$ ) (table 7.2).

\subsubsection{Definitions and diagnosis of intrauterine growth retardation}

Gestational age was estimated by $38 / 59(64.4 \%)$ participating obstetricians with the first day of the last menstrual period and by $21 / 59(35.6 \%)$ with early fetal biometry. 
Table 7.2

Response

\begin{tabular}{|c|c|c|c|c|c|c|c|c|c|c|}
\hline number of: & Hospital & 1 & 2 & 3 & 4 & 5 & 6 & 7 & 8 & total \\
\hline staff members & & 9 & 11 & 15 & 5 & 8 & 7 & 10 & 8 & 73 \\
\hline respondents & & 9 & 11 & 14 & 4 & 6 & 5 & 6 & 4 & 59 \\
\hline$\%$ response & & 100 & 100 & 93 & 80 & 75 & 71 & 60 & 50 & 81 \\
\hline
\end{tabular}

Growth retardation was defined on the basis of a delay in fundal height measurements by $41 / 54(75.9 \%)$ obstetricians, using a delay of two (21/54 $38.9 \%)$, three (18/54 $33.3 \%)$, or four or more weeks (2/54 3.7\%). Thirteen out of fifty four $(24.1 \%)$, used ultrasonographic fetal biometry to define IUGR, with threshold values of two weeks $(9 / 5416.7 \%)$, or three weeks ( $4 / 547.4 \%)$ below the average for the gestational age.

The period of gestation in which the suspicion of IUGR arises most often was indicated by $4 / 57(7.0 \%)$ to be between $24-28$ weeks, by $33 / 57$ (57.9\%) between $28-32$ weeks, by $16 / 57(28.1 \%$ ) between $32-34$ weeks, and by $4 / 57$ (7.0\%) between $34-36$ weeks of gestation.

\subsubsection{Clinical policy in suspected intrauterine growth retardation}

Responding obstetricians referred $94.3 \%$ of patients (range $=68.7 \%-100 \%$ ) for an ultrasound scan to check fetal size when IUGR was suspected clinically.

The severity of the growth deficit which would lead to maternal hospitalisation for IUGR was said to be two weeks by $12 / 53(22.6 \%)$ respondents, three weeks by $25 / 53$ (47.2\%), and four weeks by $7 / 53$ (13.2\%). Nine out of fifty-three respondents (17.0\%) would only hospitalise the patient if the growth retardation was associated with other complications, such as hypertension, proteinuria, or oedema. The average percentage of cases in which there were further complications was $42.0 \%$ (range $=31.1-53.8 \%, \mathrm{SD}=$ 18.1) in the experience of the respondents.

Releasing a patient with suspected IUGR from hospital required the absence of IUGR for $16 / 55(29.1 \%)$, while $19 / 55(34.5 \%)$ would release a patient with an estimated growth retardation of less than two weeks, $2 / 55$ (3.6\%) with less than three weeks, $9 / 55$ $(16.4 \%)$ in the absence of further complications even though a deficit of up to three weeks could still be present, and $9 / 55$ (16.4\%) would do so only after delivery.

Of the 55 respondents, $33(60 \%)$ agreed with the statement that there was a consensus with regard to the clinical policy on suspected IUGR, 20 (36.4\%) disagreed, and 2 $(3.6 \%)$ had no opinion. 
Table 7.3

Deliveries and outcome at university hospitals in the Netherlands in 1991

\begin{tabular}{llll}
\hline $\begin{array}{l}\text { Hospital } \\
\text { neliveries }\end{array}$ & $\begin{array}{l}\text { Neonates }<\text { P10 } \\
\%\end{array}$ & $\begin{array}{l}\text { Neonares }<\text { P2.3 } \\
\%\end{array}$ \\
\hline 1 & 1,458 & 13.1 & 2.5 \\
2 & 927 & 18.4 & 6.3 \\
4 & 1,226 & 13.7 & 4.3 \\
5 & 1,268 & 11.9 & 2.3 \\
6 & 1,112 & - & - \\
7 & 1,405 & - & - \\
8 & 1,376 & 13.8 & 1.7 \\
mean & 1,401 & 13.7 & 3.4 \\
total number & 10,173 & 14.1 & \\
\hline
\end{tabular}

— = percentage not known, $\mathrm{P}=$ birth weight centile

\subsubsection{Estimation of the magnitude of the problem}

Table 7.3 shows the number of singleton deliveries and neonates with a birth weight below the 10th and the 2.3rd centile (P) at each hospital over 1991, excluding intrauterine transferrals. Two centres failed to report the percentages of neonates below the 10 th and the 2.3 rd centile.

To gain an impression of the magnitude of the problem of suspected growth retardation at university hospitals, the annual number of pregnancies suspected of IUGR and the hospitalisation rates associated with this condition were estimated in two ways. Firstly, participants were asked to estimate the overall numbers directly and, secondly, they were asked to estimate the annual number of cases which they personally diagnosed and hospitalised among the patients in their care.

The overall estimates could simply be averaged. IUGR was suspected in an average of 151 patients per outpatient clinic per year (range between hospitals = 92-315). Dividing this by the number of singleton deliveries at university hospitals in 1991 resulted in an average 'prevalence' of suspected IUGR of $11.9 \%(S D=4.7)$. On average, 104 patients were estimated to be hospitalised per clinic per year for IUGR (range = 62-127), which would amount to $68.5 \%$ of all patients suspected of growth retardation (table 7.4). The sum of all the personal experience estimates should result in similar estimates. However, the response was incomplete. If the missing values of non-respondents were approximated by the mean value of the respondents for that hospital, the sum of the personal estimates averaged 117 patients per hospital (range $=56-210)$ (table 7.5). This resulted in an average prevalence of suspected IUGR of $8.0 \%$. The obstetricians were also asked to indicate the percentage of patients with suspected IUGR in their care who 
Table 7.4

Estimated 'prevalence' of suspected IUGR and the number of patients hospitalised for suspected IUGR

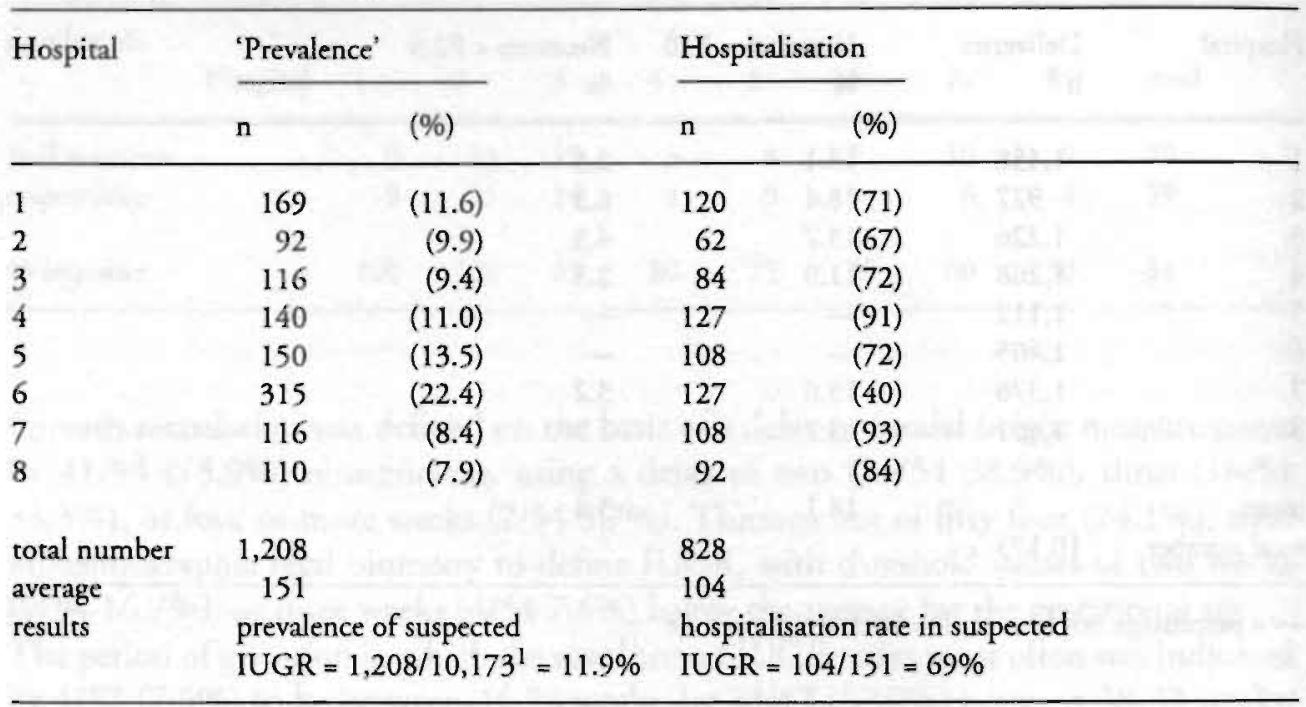

${ }^{1}$ annual number of deliveries in university hospitals (rable 7.3); data not between brackets were obtained directly from the questionnaire; data berween brackets were calculated from these estimates

Table 7.5

Personal annual estimates of 'prevalence' and the proportion of patients hospitalised in suspected IUGR

\begin{tabular}{|c|c|c|c|c|}
\hline \multirow[t]{2}{*}{ Hospital } & \multicolumn{2}{|c|}{ 'Prevalence' } & \multicolumn{2}{|c|}{ Hospitalisation } \\
\hline & $\mathrm{n}$ & $(\%)$ & (n) & $\%$ \\
\hline 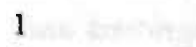 & 162 & (11.1) & (118) & 69 \\
\hline 2 & 78 & $(8.4)$ & (63) & 68 \\
\hline 3 & 56 & (4t.G) & (74) & 63 \\
\hline 4 & 181 & $(14.3)$ & $(140)$ & 100 \\
\hline 5 & 72 & $(6.5)$ & (104) & 69 \\
\hline 6 & 201 & (14.3) & (315) & 100 \\
\hline 7 & 66 & $(4.8)$ & (50) & 42 \\
\hline total number & 816 & & & \\
\hline average & 117 & & & $73 \%$ \\
\hline results & $\begin{array}{l}\text { preva } \\
\text { IUGI }\end{array}$ & $\begin{array}{l}\text { suspected } \\
110,173^{1}=8 \%\end{array}$ & $\begin{array}{l}\text { hospit } \\
\text { IUGR }\end{array}$ & on rate in suspected \\
\hline
\end{tabular}

${ }^{1}$ annual number of deliveries in university hospitals (table 7.3); data not between brackets were obtained directly from the questionnaire; data between brackets were calculated from these estimates 
were hospitalised: $100 \%, 75 \%, 50 \%, 25 \%,<25 \%$, or none. It could be calculated from their responses that an average $73 \%$ of their patients with suspected IUGR were hospitalised (range $=42-100$ ). In the centre with a low response, these personal estimates were not calculated.

\subsubsection{Doppler ultrasound measurements in the umbilical artery}

All university hospitals in the Netherlands have one or more Doppler machines at their disposal. In five university centres, Doppler results were used in clinical decision making. If the result of the Doppler was known, 28/55 (50.9\%) obstetricians felt more confident about fetal wellbeing, none felt less confident, and $27 / 55(49.1 \%)$ reported that the Doppler result would not influence their confidence.

If an abnormal measurement was found at Doppler examination in a clinically uneventful pregnancy, $23 / 59$ (39.0\%) obstetricians would not change their clinical policy, 23/59 (39.0\%) would order frequent cardiotocographic (CTG) monitoring, $13 / 59(22.0 \%)$ would increase the frequency of outpatient checkups, $7 / 59(11.9 \%)$ would hospitalise the patient. Three $(5.1 \%)$ would prefer another, unspecified clinical policy, 5 (8.5\%) had no opinion. Fifteen (25.4\%) chose more than one answer.

If Doppler measurements of the fetal circulation were routinely available, the frequency of CTGs ordered would be reduced by $5 / 54(9.3 \%)$ respondents, increased by $12 / 54$ $(22.2 \%)$, and maintained at the same level by $28 / 54(51.9 \%)$, while $9 / 54(16.7 \%)$ had no opinion. Doppler examinations were thought to be useful in IUGR, hypertension, preeclampsia, diabetes mellitus, and multiple pregnancies.

Of the 57 responding obstetricians, $26(45.6 \%)$ were of the opinion that Doppler ultrasound measurements were an asset to prenatal diagnosis, $13(22.8 \%)$ disagreed, and $18(31.6 \%)$ had no opinion.

\subsection{Discussion}

\subsubsection{Intrauterine growth retardation}

Duration of pregnancy was established in most cases on the basis of the last menstrual period, despite the proven value of early fetal biometry, which was shown to lead to a $30 \%$ reduction in suspected IUGR in a recent randomised clinical trial (Waldenström 1992). Regretfully, the period of gestation in which growth retardation was most frequently suspected, was too broadly defined in the questionnaire as a period of 28 to 34 weeks. The method primarily used to assess fetal size was measurement of fundal height (75\%). In general, the responding obstetricians defined growth retardation as a fetal size which is two or more weeks below average for the gestational age (55.6\%). The percentage increased to 96.3 at a threshold value of three weeks below average. A three week delay in the third trimester coincides with the range at which fetal biometry 
parameters fall below the 10th centile for that gestational age. Apparently, the intuitive definition of IUGR corresponds with a fetal size below the 10th centile.

When IUGR was suspected clinically, fetal biometry by ultrasound was used in nearly all cases to check fetal size. It would have been interesting to know whether the clinicians were reassured about fetal wellbeing when subsequent ultrasound measurements were within a normal range.

A delay in estimated fetal size of three weeks or more by any parameter should lead to hospitalisation in the opinion of in $69.8 \%$ of the obstetricians. Opinions about the criteria for discharge of a patient were almost evenly divided between a growth retardation of less than two weeks (34.5\%) and its total absence (29.1\%). However, none of the suggested criteria received great support, indicating poor consensus.

The high rate of further complications $(42.0 \%, \mathrm{SD}=18.1 \%)$ in the experience of the obstetricians illustrates that IUGR is an important obstetric problem.

The estimated 'prevalence' of patients with suspected IUGR was in the order of $11 \%$ of singleton pregnancies (table 7.4). This is in agreement with percentages in the literature (Stembera 1985). The percentage is in between the percentages of neonates with birth weights below the 10 th and the 2.3 rd centiles (table 7.3 ). Nearly $70 \%(S D=16.1)$ of these patients were estimated to be hospitalised per year. Summed estimations per hospital of cases in the personal experience of the obstetricians (table 7.5) are not reliable as the response was incomplete, but with a prevalence of $8 \%$ and a hospitalisation rate of $73 \%$, they support the general size of the direct estimates and give a rough idea of the numbers of patients involved.

In summary, approximately 830 pregnant women are hospitalised every year at the eight university clinics because growth retardation is suspected. As was stated in the introduction, a proportion of these theoretically concern healthy, genetically small fetuses. Therefore, the possible benefits of a more selective hospitalisation policy in suspected IUGR could be considerable, especially when applied on a national scale (table 7.6).

\section{Table 7.6}

Estimated annual magnitude of suspected IUGR in the Netherlands

$\begin{array}{lll}\text { Suspected IUGR Number of cases } \% \text { of previous } & \begin{array}{l}\% \text { of university } \\ \text { hospital population }\end{array}\end{array}$

\begin{tabular}{lrrr}
\hline singleton births & $193,780^{1}$ & $100 \%$ & - \\
suspected of IUGR & 23,254 & $12 \%$ & $12 \%$ \\
hospitalised & 16,045 & $69 \%$ & $8 \%$
\end{tabular}

${ }^{1}$ singleton birchs over 28 weeks of gestation in the Netherlands, data 1991 (CBS 1993) 


\subsubsection{Doppler ultrasound measurements in the umbilical artery}

To our knowledge, two questionnaires have assessed the use of Doppler ultrasound measurements in obstetrics, one in university and reaching hospitals in German speaking countries (Schneider 1993) and one which surveyed the indications for fetal Doppler in six French obstetric departments (Rochambeau 1992).

All university departments of Obstetrics in the Netherlands had access to Doppler machines. Five hospitals used Doppler ultrasound measurements in clinical decision making. Of the responding obstetricians, $45.6 \%$ thought Doppler measurements an asset to prenatal diagnosis. In the departments where Doppler measurements were applied in clinical decision making, $75.0 \%$ of obstetricians were in favour, against $29.8 \%$ in departments were Doppler measurements were not used in patient care. It is not known whether a favourable attitude towards the clinical value of Doppler ultrasound leads to acquisition of a Doppler machine or whether personal experience with Doppler ultrasound influences the attitude. All 44 responding university hospitals in German speaking countries had access to Doppler machines. Of the 144 responding university and teaching hospitals in German speaking countries, 51 (35.4\%) thought that Doppler ultrasound had an additional diagnostic value. The answers given by the German and French studies corresponded with those provided by the present Dutch survey on the indications for Doppler measurements, namely IUGR, (pregnancy induced) hypertension, multiple pregnancy, diabetes, and other high risk pregnancies.

Opinions were almost equally divided on the question whether Doppler ultrasound measurements did $(50.9 \%)$ or did not $(49.1 \%)$ provide reassurance about fetal wellbeing. The respondents were also equally divided on the question whether they would change the frequency of CTG monitoring in cases of raised umbilical artery Doppler values: $39 \%$ would increase its frequency and the same percentage would not. A recent randomised clinical trial (Almström 1992) suggested that Doppler measurements could replace CTG as a monitoring technique of fetal wellbeing in suspected IUGR. According to the present survey, obstetricians would not exchange CTG for Doppler ultrasound in fetal monitoring.

With regard to the clinical value of ultrasonic fetal biometry and Doppler ultrasound blood velocity measurements in pregnancies suspected of intrauterine growth retardation, an investigation of their costs, and their additional value in differentiating low and high risk pregnancies is warranted. As umbilical artery blood flow velocity profiles can be easily assessed, even in case of severely reduced amniotic fluid, Doppler ultrasound measurements might prove more reliable than fetal biometry in the identification of the haemodynamic aetiology of intrauterine growth retardation. 


\subsection{Conclusion}

The results obtained by questionnaires are naturally 'sof', but they do give a general picture of what actually happens in clinical practice after the diagnosis suspected intrauterine growth retardation is made, a diagnosis that 'disappears' after delivery if it proves incorrect by neonatal outcome.

Sixty percent of the responding obstetricians agreed with the statement that there was a consensus clinical policy on suspected IUGR. This was in agreement with the distribution of the answers to the other questions on diagnosis, clinical policy, and the use of Doppler ultrasound in suspected intrauterine growth retardation. The responding obstetricians did not unanimously agree with any of the answers, but to most of the questions there was an answer preferred by the majority. The general attitude towards Doppler measurements can be roughly summarised as 'fifty-fifty'.

\section{Acknowledgements}

We wish to thank all participating obstetricians, particularly those who acted as contact persons and actively stimulated the completion of the questionnaires, resulting in a sizable response rate. 


\section{References}

Albertsson-Wikjand K, Wennergren G, Wennergren M, Vilbergsson G, Rosberg S. Longitudinal follow-up of growth in children born small for gestational age. Acta Paediatr 1993; 82: 438-43.

Almström H, Axelsson O, Cnattingius S, Ekman G, Maesel A, Ulmsten U, Årström K. Comparison of umbilical-artery velocimetry and cardiotocography for surveillance of small-for-gestarional-age fetuses. Lancet 1992; 340: 936-40.

Altman DG, Hytten FE. Intrauterine growth retardation. Let's be clear about it. Br J Obstet Gynaecol 1989; 96: 1127-32.

Ament AJHA. Het stellen van prioriteiten in de gezondheidszorg: Het selecteren van waardevolle medische interventies en protocollen. Nederlands Tijdschr Obstet Gynaecol, in press 1995.

Amiel-Tison C, Grenier A. Neurological assessment during the first year of life. Oxford University Press, 1986.

Amiel-Tison C, Pettigrew AG. Adaptive changes in the developing brain during intrauterine stress. Brain Dev 1991; 13: 67-76.

Arduini D, Rizzo G, Romanini C, Mancuso S. Fetal blood flow velocity waveforms as predictors of growth retardation. Obstet Gynecol 1987; 70: 7-10.

Arduini D, Rizzo G. Normal values of Pulsatility Index from fetal vessels: a cross-sectional study on 1556 healthy fetuses. J Perinat Med 1990; 18: 165-72.

Årström K, Eliasson A, Hareide JH, Maršál K. Fetal blood velocity waveforms in normal pregnancies. A longitudinal study. Acta Obstet Gynecol Scand 1989; 68: 171-8.

Bakketeig LS, Hoffman HJ. The tendency to repeat gestational age and birth weight in successive births related to perinatal survival. Acta Obster Gynecol Scand 1983; 62: 383-92.

Barker DJP, Bull AR, Osmond C, Simmonds SJ. Fetal and placental size and the risk of hypertension in adult life. Br Med J 1990; 301: 259-62.

Barker DJ. The fetal origins of adult hypertension. J Hypertens 1992; 10 (Suppl 7): 39-44.

Bartelds JF, Jansen EPWA, Joostens ThH. Enquêteren: het opstellen en gebruiken van vragenlijsten. Wolters-Noordhoff, 1989.

Beattie RB, Dornan JC. Antenatal screening for intrauterine growth retardation with umbilical artery Doppler ultrasonography. Br Med J 1989; 11: 631-5.

Belizán JM, Villar J, Nardin JC, Malamud J, Sainz de Vicuna L. Diagnosis of intrauterine growth retardation by a simple clinical method: Measurement of uterine height. Am J Obstet Gynecol 1978; 131 : 643-6. 
Belizán JM, Villar J, Nardin JC. Poor predictive value of symphysial-fundal height when misused in clinical practice. Am J Obstet Gynecol 1990; 162: 1348-9.

Berkowitz GS, Mehalek KE, Chitkara U, Rosenberg J, Cogswell C, Berkowitz RL. Doppler umbilical velocimetry in the prediction of adverse outcome in pregnancies at risk for intrauterine growth retardation. Obstet Gynecol 1988; 71: 742-6.

Bolte A, Fuhrman U, Hamm W, Schlensker KH, Stenzel B. Geburtshilfliches Management bei schwerer fetaler Wachstumsretardierung. Ergebnisbericht anhand von 278 Neugeborenen mit schwerer Dystrophie der Jahrgänge 1970-1985. Geburtsh Frauenheilk 1987; 47: 518-24.

Bossi A, Milani S. Italian standards for crown-heel length and head circumference at birth. Ann Hum Biol 1987; 14: 321-35.

Bouter LM, Dongen MCJM van. Epidemiologisch onderzoek: opzet en interpretatic. Bohn, Scheltema en Holkema, 1988.

Bowes WA. Obstet Gynecol Surv 1993; 48: 402-5. On: Davies JA, Gallivan S, Spencer JAD. Randomised controlled trial of Doppler ultrasound screening of placental perfusion during pregnancy. Lancet 1992; 340: 1299-1303.

Bracero LA, Beneck D, Kirshenbaum N, Peiffer M, Stalter P, Schulman H. Doppler velocimetry and placental disease. Am J Obstet Gynecol 1989; 161: 388-93.

Brandt I. Brain growth, fecal malnutrition, and clinical consequences. J Perinat Med 1981; 9: 3-26.

Brandt I. Growth dynamics of low-birth-weight infants with emphasis on the perinatal period. In: F Falkner, JM Tanner (eds). Human growth. A comprehensive treatise. Plenum Press, 1986; vol. 1, chapter 21: 415-75.

Brandt I, Sticker EJ. Bedeutung der Alterskorrektur bei Frühgeborenen. Monatsschr Kinderheilk 1991; 139: 16-21.

Brosens I, Dixon HG, Robertson WB. Fetal growth retardation and the arteries of the placental bed. $\mathrm{Br}$ ] Obstet Gynaecol 1977; 84: 656-63.

Burke G, Stuart B, Crowley P, Scanaill SN, Drumm J. Is intrauterine growth retardation with normal umbilical artery blood flow a benign condition? Br Med J 1990; 300: 1044-5.

Calvert JP, Crean EE, Newcombe RG, Pearson JF. Antenatal screening by measurement of symphysis-fundus height. Br Med J 1982; 285: 846-9.

Campbell MJ, Machin D. Medical statistics. A commonsense approach. John Whiley \& Sons, Chichester 1990: 121-2.

Casey PH, Kraemer HC, Bernbaum J, Tyson JE, Sells JC, Yogman MW, Bauer CR. Growth patterns of low birth weight preterm infants: a longitudinal analysis of a large, varied sample. J Pediatr 1990; 117: 298-307.

CBS. Statistisch Jaarboek 1993. SDU/Uitgeverij CBS-publicaties, 's-Gravenhage 1993: 36.

Chalmers I, Hetherington J, Elbourne D, Keirse MJNC, Enkin M. Materials and methods used in synthesizing evidence to evaluate the effecrs of care during pregnancy and childbirth. In: I Chalmers, M Enkin, MJNC Keirse (eds). Effective care in pregnancy and childhood. Oxford University Press, 1989; vol. 1 , chapter 2 . 
Chambers SE, Hoskins PR, Haddad NG, Johnstone FD, McDicken WN, Muir BB. A comparison of feral abdominal circumference measurements and Doppler ultrasound in the prediction of small-for-date babies and fetal compromise. Br J Obstet Gynaecol 1989; 96: 803-8.

Chambers HM, Knowles S, Staples A, Tamblyn M, Haan EA. Anthropometric measurements in rhe second trimester ferus. Early Hum Dev 1993; 33: 45-59.

Chiswick ML. Intrauterine growth retardation. Br Med J 1985; 291: 845-8.

Clapp JF, Szeto HH, Larrow R, Hewitt J, Mann LI. Umbilical blood flow response to embolization of the uterine circulation. Am J Obstet Gynecol 1980; 138: 60-7.

Clark SL, Cotton DB, Lee W, Bishop C, Hill T, Southwick J, Pivarnik J, Spillman T, DeVore GR, Phelan J, Hankins GDV, Benedetti TJ, Tolley D. Central hemodynamic assessment of normal term pregnancy. Am J Obstet Gynecol 1989; 161: 1439-42.

CLASP Collaborative Group. CLASP: a randomised trial of low-dose aspirin for the prevention and treatment of pre-eclampsia among 9364 pregnant women. Lancet 1994; 343: 619-29.

Cnattingius S. Antenatal screening for small-for-gestational-age, using risk factors and measurements of the symphysis-fundus distance - 6 years of experience. Early Hum Dev 1988; 18: 191-7.

Commey JOO, Fizhardinge PM. Handicap in the preterm small-for-gestational age infant. J Pediatr 1979; 94: 779-86.

Cortinovis I, Bossi A, Milani S. Longitudinal growth charts for weight, length and head circumference of Italian children up to three years. Acta Medica Auxologica 1993; 25: 13-29.

Crowther C, Chalmers I. Bed rest and hospitalization during pregnancy. In: I Chalmers, M Enkin, MJNC Keirse (eds). Effective care in pregnancy and childbirth. Oxford University Press, 1989; vol. 1: 625-8.

Cruise MO. A longitudinal study of the growth of low birth weight infants. I. Velocity and distance growth, birth to 3 years. Pediatrics 1973; 51: 620-8.

Davies DP, Platts P, Pritchard JM, Wilkinson PW. Nutritional status of light-for-date infants at birth and its influence on early postnatal growth. Arch Dis Child 1979a; 54: 703-6.

Davies DP, Beverly D. Changes in body proportions over the first year of life: comparisons berween 'light-for-dates' and 'appropriate-for-dates' term infants. Early Hum Dev 1979b; 3: 263-5.

Davies DP. Size at birth and growth in the first year of life of babies who are overweight and underweight at birth. Proc Nutr Soc 1980; 39: 25-33.

Davies DP. Growth of 'small-for-dates' babies. Early Hum Dev 1981; 1: 95-105.

Davies JA, Gallivan S, Spencer JAD. Randomised controlled trial of Doppler ultrasound screening of placental perfusion during pregnancy. Lancet 1992; 340: 1299-1303.

Dempster J, Mires GJ, Taylor DJ, Patel NB. Fetal umbilical artery flow velocity waveforms: prediction of small for gestational age infants and late decelerations in labour. Eur J Obstet Gynecol Reprod Biol 1988; 29: $21-5$.

Devoe LD, Gardner P, Dear C, Faircloth D. The significance of increasing umbilical artery systolic-diastolic ratios in third-trimester pregnancy. Obstet Gynecol 1992; 80: 684-7. 
Divon MY, Guidetti DA, Braverman JJ, Oberlander E, Langer O, Merkatz IR. Intrauterine growth retardation - a prospective study of the diagnostic value of real-time sonography combined with umbilical artery flow velocimetry. Obstet Gynecol 1988; 72:611-4.

Divon MY, Girz BA, Lieblich R, Langer O. Clinical management of the fetus with markedly diminished umbilical artery end-diastolic flow. Am J Obstet Gynecol 1989; 161: 1523-7.

Dobbing J, Sands J. Vulnerability of the developing brain. IX. The effect of nutritional growth retardation on the timing of the brain growth spurt. Biol Neonate 1971; 19:363-78.

Dobbing J, Sands J. Quantitative growth and development of human brain. Arch Dis Child 1973; 48: 757-67.

Drummond MF. Principles of economic appraisal in health care. Oxford University Press, 1987.

Dunn PM. A perinatal growth chart for international reference. Acta Paediatr Scand 1985: Suppl. 319; 180-7.

Dunn HG, Hughes C-J, Schulzer M. Physical growth. In: HG Dunn (ed.). Sequelae of low birthweight. The Vancouver study. MacKeith Press. Clin Dev Med 1986; no.95/96, chapter 3.

Duvekot JJ, Cheriex EC, Pieters FAA, Menheere PPCA, Peeters LLH. Early pregnancy changes in hemodynamics and volume homeostasis are consecutive adjustments triggered by a primary fall in systemic vascular tone. Am J Obstet Gynecol 1993; 169: 1382-92.

Falkner F. Key issues in perinatal growth. Acta Paediatr Scand 1985; Suppl. 319: 21-5.

Fergusson DM, Horwood LJ, Shannon FT. Length and weight gain in the first three months of life. Hum Biol 1980; 52: 169-80.

FitzGerald DE, Drumm JE. Non-invasive measurements of fetal circulation using ultrasound; a new mechod. Br Med J 1977; ii: 1450-1.

Fitzhardinge PM, Inwood S. Long-term growth in small-for-date children. Acta Paediatr Scand Suppl 1989; 349: 27-33.

Fleischer A, Schulman H, Farmakides G, Bracero L, Blattner P, Randolph G. Umbilical artery velocity waveforms and intrauterine growth retardation. Am J Obstet Gynecol 1985; 151: 502-5.

Fogarty P, Beatrie B, Harper A, Dornan J. Continuous wave Doppler flow velocity waveforms from the umbilical artery in normal pregnancy. J Perinat Med 1990; 18: 51-7.

Fox H. Pathology of the placenta. Clin Obster Gynaecol 1986; 13: 501-19.

Geirsson RT, Patel NB, Christie AD. Efficacy of intrauterine volume, fetal abdominal area and biparietal diameter measurements with ultrasound in screening for small-for-dates babies. Br J Obster Gynaecol 1985; 92: 929-35.

Gennser G, Rymark P, Isberg PE. Low birth weight and risk of high blood pressure in adulthood. Br Med J Clin Res 1988; 296: 1498-500.

Gerver WJM. Measurements of the body proportions in children. The Oosterwolde study. Thesis, Groningen, 1988.

Gerver WJM, Gidding C, Amstel M van, Vles JSH. A new device to measure short-term growth of head circumference in newborn infants. Eur J Pediatr 1990; 149: 768-70. 
Giles WB, Trudinger BJ, Baird PJ. Fetal umbilical artery flow velocity waveforms and placental resistance: pathologic correlation. Br J Obstet Gynaecol 1985; 92: 31 -8.

Goldenberg RL, Cutter GR, Hoffman HJ, Foster JM, Nelson KG, Hauth JC. Intrauterine growch retardation: standards for diagnosis. Am J Obstet Gynecol 1989; 161: 271-7.

Goldstein H. Factors relating to birth weight and perinatal mortality. Br Med Bull 1981; 37:259-64.

Gosling RG, King DH. Processing arterial Doppler signals for clinical data. Ultrasonic angiology. In: AW Harcus, L Adamson (eds). Arteries and veins. Churchill Livingstone, 1975: 61-98.

Grant A. Principles for clinical evaluation of methods of perinatal monitoring. J Perinat Med 1984; 12: 227-33.

Gross TL, Sokol RJ. Intrauterine growth retardation: a practical approach. Year Book Medical Publishers, 1989.

Gruenwald P. Growth of the human fetus. I. Normal growth and its variation. Am J Obstet Gynecol 1966; 94: 1112-9.

Gudmundsson S, Maršal K. Umbilical artery and uteroplacental blood flow velocity waveforms in normal pregnancy: a cross-sectional study. Acta Obstet Gynecol Scand 1988; 67: 347-54.

Guo S, Roche AF, Yeung DL. Monthly growth status from a longitudinal study of Canadian infants. Can J Public Health 1990; 81:215-21.

Guo S, Roche AF, Fomon SJ, Nelson SE, Chumlea WC, Rogers RR, Baumgarmer RN, Ziegler EE, Siervogel RM. Reference data on gains in weight and length during the first two years of life. J Pediatr 1991: 119; 355-62.

Haddad NG, Johnstone FD, Hoskins PR, Chambers SE, Muir BB, McDicken WN. Umbilical artery Doppler waveform in pregnancies with uncomplicated incra-uterine growth retardation. Gynecol Obstet Invest 1988; 26: 206-10.

Hadders-Algra M. Correlates of brain dysfunction in children - a follow-up study. Thesis, Groningen, 1987.

Hagberg B, Hagberg G. Decreasing perinatal mortality - Increase in cerebral palsy morbidity? Acta Paediatr Scand 1989; 78: 664-70.

Hanretty KP, Primrose MH, Neilson JP, Whittle MJ. Pregnancy screening by Doppler uteroplacental and umbilical artery waveforms. Br J Obstet Gynaecol 1989; 96: 1163-7.

Hasaart THM, Maulik D, Morrow RJ. Validation of fetal flow velocimetry: a review of in vitro and in vivo modeling. J Matern Fetal Invest 1993; 3: 95-104.

Heinonen K, Matilianen R, Koski H, Launiala K. Intrauterine growth retardation (IUGR) in premterm infants. J Perinat Med 1985; 13: 171-8.

Herman AA, Yu KF, Hoffman HJ, Krulewitch CJ, Bakketeig LS. Birth weight, gestational age and perinatal mortality: biological heterogeneity and measurement error. Early Hum Dev 1993; 33: 29-44.

Herschkowiz N. Brain development in the fetus, neonate and infant. Biol Neonate 1988; 54: 1-19.

Hitchcock NE, Coy JF. Growth and catch-up growth of Australian infants of low birthweight. Aust Paediatr J 1989; 25: 134-7. 
Hofmeyr GJ, Pattinson R, Buckley C, Jennings J, Redman G. Umbilical artery Resistance Index as a screening test for fetal well-being. II. Randomised feasibility study. Obstet Gynecol 1991; 78: 359-62.

James DK, Parker MJ, Smoleniec JS. Comprehensive fetal assessment with three ultrasonographic characteristics. Am J Obstet Gynecol 1992; 166: 1486-95.

Jensen $\mathrm{OH}$, Larsen S. Evaluation of symphysis-fundus measurements and weighing during pregnancy. Acta Obstet Gynecol Scand 1991a; 70: 13-6.

Jensen OH, Guimaraes MS. Prediction of fetal outcome by Doppler examination and by non-stress test. Acta Obstet Scand 1991b; 70: 271-4.

Johnstone FD, Prescott R, Hoskins P, Greer IA, McGlew T, Comton M. The effect of introduction of umbilical Doppler recordings to obstetric practice. $\mathrm{Br}$ J Obstet Gynaecol 1993; 100: 733-41.

Jones RAK, Roberton NRC. Problems of the small-for-dates baby. Clin Obstet Gynecol 1984; 11: 499-524.

Karlberg P, Engström I, Lichtenstein H, Svennberg I. The development of children in a Swedish urban community. A prospective longitudinal study. III. Physical growth during the first three years of life. Acta Paediatr Scand 1968; Suppl 187: 48-66.

Keen DV, Pearce RG. Weight, length, and head circumference curves for boys and girls of between 20 and 42 weeks' gestation. Arch Dis Child 1988; 63: 1170-2.

Keirse MJNC. Aetiology of intrauterine growth retardation. In: FA van Assche, WB Robertson, M Renaer (eds). Fetal growth retardation. Churchill Livingstone, 1981: 37-56.

Khong TY, Wolf F de, Robertson WB, Brosens I. Inadequate maternal vascular response to placentation in pregnancies complicated by pre-eclampsia and by small-for-gestational age infants. Br Med J 1986; 93 : 1049-59.

Kloosterman GJ. On intrauterine growth. Int J Gynaecol Obstet 1970; 8: 895-912.

Knottnerus JA. De evaluatie van diagnostische technieken. In: JDF Habbema, AF Casparie, JH Mulder, FFH Rutten (eds). Medische technologie assessment en gezondheidsbeleid. Samson Stafleu, 1989: 76-91.

Koops BL, Morgan LJ, Battaglia FC. Neonatal mortality risk in relation to birth weight and gestational age: update. J Pediatr 1982; 101: 969-77.

Korner AF. The scope and limitations of neurologic and behavioral assessments of the newborn. In: DK Stevenson, P Sunshine (ed.). Fetal and neonatal brain injury. Mechanism, management and the risks of practice, BC Decker Inc, 1989; chaprer 19: 239-49.

Kramer PD, Constan D, Kreminski J, Brondy D, Martin C. Hospitalization on the high risk maternity unit. Gen Hosp Psychiatry 1986; 8: 33-9.

Kramer MS. Intrauterine growth and gestational duration determinants. Pediatrics 1987; 80: 502-11.

Kramer MS, McLean FH, Olivier M, Willis DM, Usher RH. Body proportionality and head and length 'sparing' in growth-retarded neonates: a critical reappraisal. Pediatrics 1989; 84: 717-23.

Kranen VHJM van-Mastenbroek. Spontaneous motor behaviour in full-term small for gestational age and appropriate for gestational age newborn infants. Thesis, Maastricht, 1993.

Kurmanavichius J, Baumann H, Huch R, Huch A. Determination of the minimum number of cardiac cycles necessary to ensure representative blood flow velocity measurements. J Perinat Med 1989; 17: 33-9. 
Largo RH, Wälli R, Duc G, Fanconi A, Prader A. Evaluation of perinatal growth. Helv Paediatr Acta 1980; 35: 419-36.

Laurin J, Persson PH. Ultrasound screening for detection of intra-uterine growth retardation. Acta Obstet Gynecol Scand 1987a; 66: 493-500.

Laurin ], Persson PH. The effect of bedrest in hospital on fetal outcome in pregnancies complicated by intra-uterine growth retardation. Acta Obstet Gynecol Scand 1987b; 66: 407-11.

Lavery JP. The human placenta: clinical perspectives. Aspen Publishers, Inc., 1987.

Lawrence C, Fryer JG, Karlberg P, Niklasson A, Ericson A. Modelling of reference values for size at birth. Acra Paediatr Suppl 1989; 350: 55-69.

Legarth J, Thorup E. Characteristics of Doppler blood-velocity waveforms in a cardiovascular in vitro model. I. The model and the influence of pulse rate. Scand J Clin Lab Invest 1989; 49: 451-7.

Lems W, Hopkins B, Samsom JF. Mental and motor development in preterm infants: the issue of corrected age. Early Hum Dev 1993; 34: 113-23.

Lin CC, Evans MI. Intrauterine growth retardation: pathophysiology and clinical management. McGrawHill Book Company, 1984.

Lindgren R, Selbing A, Leander E. Which fetal growth charts should be used? Acta Obster Gynecol Scand 1988; 67: 683-7.

Lindhard A, Nielsen PV, Mouritsen LA, Zachariassen A, Sorensen HU, Roseno H. The implications of introducing the symphyseal-fundal height-measurement. A prospective randomized controlled trial. $\mathrm{Br}$ J Obstet Gynaecol 1990; 97: 675-80.

Longo LD. Intrauterine growth retardation: a 'mosaic' hyporhesis of pathophysiology. Semin Perinat 1984; 8: 62-72.

Lowery CL Jr, Henson BV, Wan J, Brumfield CG. A comparison between umbilical artery velocimetry and standard antepartum surveillance in hospitalised high-risk patients. Am J Obstet Gynecol 1990; 162: $710-4$.

Lubchenco LO, Hansman C, Dressler M, Boyd E. Intrauterine growths as estimated from lifeborn birth weight data at 24 to 42 weeks of gestation. Pediatrics 1963; 32: 793-800.

Mason GC, Lilford RJ, Porter J, Nelson E, Tyrell S. Randomised comparison of rourine versus highly selective use of Doppler ultrasound in low risk pregnancies. Br J Obstet Gynaecol 1993; 100: 130-3.

Maulik D, Yarlagadda P, Youngblood JP, Ciston P. The diagnostic efficacy of the umbilical arterial systolic/diastolic ratio as a screening tool: a prospective blinded study. Am J Obster Gynecol 1990; 162: 1518-23.

McCowan LM, Mullen BM, Ritchie K. Umbilical artery flow velocity waveforms and the placental vascular bed. Am J Obstet Gynecol 1987; 157: 900-2.

McParland P, Pearce JM. Doppler blood flow in pregnancy. Placenta 1988; 9: 427-50.

Mendez $\mathrm{H}$. Introduction to the study of pre- and postnatal growth in humans: a review. Am J Med Gen 1985; 20: 63-85.

Michaelis R, Asenbauer C, Buchwald-Saal M, Haas G, Krägeloh-Mann I. Transitory neurological findings in a population of at risk infants. Early Hum Dev 1993; 34: 143-53. 
Miller HC. Prenatal factors affecting intrauterine growth retardation. Clin Perinatol 1985; 12: 307-18.

Miller JM, Gabert HA. Comparison of dynamic image and pulsed Doppler ultrasonography for the diagnosis of the small-for-gestational-age fetus. Am J Obstet Gynecol 1992; 166: 1820-6.

Moar VA, Ounsted MK. Growth in the first year of life: how early can one predict size at twelve months among small-for-dates and large-for-dates babies? Early Hum Dev 1982; 6: 65-9.

Mohide P, Grant A. Evaluating diagnosis and screening during pregnancy and childbirch. In: I Chalmers, M Enkin, MJNC Keirse (eds). Effective care in pregnancy and childbirth. Oxford University Press, 1989; vol. 1: 66-80.

Myers SA, Ferguson R. A population study of the relationship between fetal death and altered fetal growth. Obstet Gynecol 1989; 74: 325-31.

Neilson JP, Munjanja SP, Whitfield CR. Screening for small for dates fetuses: a controlled trial. Br Med J 1984a; 289: 1179-82.

Neilson JP, Munjanja SP, Mooney R, Whitfield CR. Product of crown-rump length and trunk area: ultrasound measurement in high-risk pregnancies. $\mathrm{Br} J$ Obstet Gynaecol 1984b; 91: 756-61.

Nelson K, Ellenberg JH. Neonatal signs as predictors of cerebral palsy. Pediatrics 1979; 64: 225-32.

Nelson KB, Ellenberg JH. Children who 'outgrew' cerebral palsy. Pediatrics 1982; 69: 529-36.

Newnham JP, Patterson LL, James IR, Diepeveen DA, Reid SE. An evaluation of the efficacy of Doppler flow velocity waveform analysis as a screening test in pregnancy. Am J Obstet Gynecol 1990; 162:403-10.

Newnham JP, O'Dea MRA, Reid KP, Diepeveen DA. Doppler flow velocity waveform analysis in high risk pregnancies: a randomised controlled trial. Br J Obstet Gynaecol 1991; 98: 956-63.

Newnham JP, Evans SF, Michael CA, Stanley FJ, Landau LI. Effects of frequent ultrasound during pregnancy: a randomised controlled trial. Lancet 1993; 342: 887-91.

Nienhuis SJ, Vugt JMG van, Hoogland HJ, Ruissen CJ, Haan J de. Interexaminer variability of fetal Doppler velocity waveforms. Gynecol Obstet Invest 1988; 25: 152-7.

Nienhuis SJ, Zusterzeel NMA], Hoogland HJ. Routine clinical policy and application of Doppler measurements in suspected intrauterine growth retardation in university hospitals in the Netherlands. Eur J Obstet Gynecol Reprod Biol 1994; 56: 31-6.

Omtzigt AWJ. Clinical value of umbilical Doppler velocimetry. Thesis, Utrecht, 1990.

Ott WJ. Comparison of dynamic image and pulsed Doppler ultrasonography for the diagnosis of intrauterine growth retardation. J Clin Ultrasound 1990; 18: 3-7.

Ott WJ. Value of fetal umbilical artery and carotid flow studies in the evaluation of suspected intrauterine growth retardation. J Matern Fetal Invest 1991; 1: 185-90.

Ouden den L, Rijken M, Brand R, Verloove-Vanhoorick SP, Ruys J. Is it correct to correct? Developmetal milestones in 555 'normal' preterm infants compared with term infants. J Pediatr 1991; 118: 399-404.

Ounsted M, Moar V, Scott A. Growth in the first four years. II. Diversity within groups of small-for-dates and large-for-dates. Early Hum Dev 1982a; 7: 29-39.

Ounsted MK, Moar V, Scott A. Growth in the first year of life: Effects of sex and weight for gestational age at birth. Develop Med Child Neurol 1982b;24:356-65. 
Ounsted M. Small-for-dates babies: heterogeneity and the concept of 'brain sparing'. Paediatr Perinat Epidemiol 1988; 2: 365-70.

Pattinson R, Dawes G, Jennings J, Redman C. Umbilical artery Resistance Index as a screening test for fetal well-being. I. Prospective revealed evaluation. Obstet Gynecol 1991; 78: 353-8.

Pearce JM, Campbell S. A comparison of symphysis-fundal height and ultrasound as screening test for light-for-gestational age infants. Br J Obstet Gynaecol 1987; 94: 100-4.

Pearce JM, Campbell S, Cohen-Overbeek T, Hackett G, Hernandez J, Royston JP. Reference ranges and sources of variation for indices of pulsed Doppler flow velocity waveforms from the uteroplacental and fetal circulation. Br J Obstet Gynaecol 1988; 95: 248-56.

Peeters LLH, Sheldon RE, Jones MD, Makowski EL, Meschisa G. Blood flow to fetal organs as a function of arterial oxygen content. Am J Obstet Gynecol 1979; 135: 637-46.

Persson B, Stangenberg M, Lunell NO, Brodin U, Holmberg NG, Vaclavinkova V. Prediction of size of infants at birth by measurement of symphysis fundus height. $\mathrm{Br} J$ Obstet Gynaecol 1986; 93: 206-11.

Petersen S, Larsen T, Greisen G. Fetal abdominal circumference rather than fetal femur length/abdominal circumference ratio predicts fetal malnutrition in high risk pregnancies. J Perinat Med 1989; 17: 439-45.

Pocock SJ. Clinical Trials. A practical approach. John Whiley \& Sons Ltd, 1983.

Potter EL, Craig J. Pathology of the fetus and infant. Year Book Medical Publishers, 1975: 18.

Pourcelot L. L'examen Doppler des vaisseaux peripheriques. AC-D Productions, 1982.

Prader A, Largo RH, Molinari L, Issler C. Physical growth of Swiss children from birth to 20 years of age. Helvetica Paediatrica Acta Suppl 52, 1988; 43: 1-125.

Prechtl HFR. The neurologic examination of the full term newborn infant. Clin Dev Med 1977; No. 63: 63-8.

Preece MA. The anthropometric considerations in the evaluation of growth promoting treatments. In: MB Ranke, JR Bierich. Pediatric endocrinology: past, present and future. MD-Verlag Munich, 1986: 22-8.

Quaranta P, Currel R, Redman CWG, Robinson JS. Prediction of small-for-dates infants by measurement of symphysial-fundal-height. $\mathrm{Br} \mathrm{J}$ Obstet Gynaecol 1981; 88: 115-9.

Reuwer PJHM, Nuyen WC, Beijer HJM, Heethaar RM, Haspels AA, Bruinse HW. Feto-placental circulatory competence. Eur J Obstet Gynecol Reprod Biol 1986; 21: 15-26.

Reuwer PJHM, Sijmons EA, Rietman GW, Tiel MWM van, Bruinse HW. Intrauterine growth retardation: prediction of perinatal distress by Doppler ultrasound. Lancet 1987 ; ii: 415-8.

Ribbert LSM. Assessment of fetal well-being in growth retardation. Thesis, Groningen, 1993.

Robertson WB, Brosens IA, Dixon HG. Maternal blood supply in fetal growth retardation. In: FA van Assche, WB Robertson, M Renaer (eds). Fetal Growth Retardation. Churchill Livingstone, 1981: 126-38.

Rochambeau B de, Fignon A. Use of fetal Doppler in routine obstetrics: the point of view of the French obstetrician. J Matern Fetal Invest 1992; 2: 71-3.

Roede MJ, Wieringen JC van. Growth diagrams 1980. T Sociale Gezondheidszorg Suppl 1985; 63: 1-34.

Rohrer F. Der Index der Körperfülle als Mass des Ernährungszustandes. Munchener Medizinische Wochenschrift 1921; 68: 580-2. 
Rooth G, Meirik O, Karlberg P. Estimation of the 'normal' growth of Swedish infants at term. Preliminary report. Acta Paediatr Scand Suppl 1985; 319: 76-9.

Rosenberg K, Grant JM, Tweedie I, Aitchison T, Gallagher F. Measurement of fundal height as a screening rest for fetal growth retardation. Br J Obstet Gynaecol 1982; 89: 447-50.

Rosendahl H, Kivinen S. Routine ultrasound screening for early detection of small for gestational age fetuses. Obstet Gynecol 1988; 71: 518-21.

Rubin RA, Balow B. Infant neurological abnormalities as indicators of cognitive impairment. Develop Med Child Neurol 1980; 22: 336-43.

Ruissen CJ, Vugt JMG van, Hoogland HJ, Hoeks APG, Haan J de. Technical aspects of fetal Doppler measurements. Gynecol Obstet Invest 1987; 24: 1-13.

Ruissen CJ, Drongelen MMHP van, Hoogland HJ, Hoeks APG. Characteristics of the umbilical artery velocity waveform as function of measurement site. Gynecol Obstet Invest 1990; 30: 212-6.

Rutten FFH, Ineveld BM van, Ommen R van, Hout BA van, Huijsman R. Kostenberekening bij gezondheidszorgonderzoek. Richtlijnen voor de praktijk. Uitgeverij Jan van Arkel, 1993: 13-6.

Salvesen KA, Bakketeig LS, Eik-Ness SH, Undheim JO, Okland O. Routine ultrasonography in utero and school performance at age 8-9 years. Lancet 1992; 339: 85-9.

Salvesen KA, Vatten LJ, Eik-Ness SH, Hughdahl K, Bakketeig LS. Routine ultrasonography in utero and subsequent handedness and neurological development. Br Med J 1993; 307: 159-64.

Sarma MKJ, Rao KS. Biochemical composition of different regions in the brain of small-for-date infants. J Neurochem 1974; 22: 671-7.

Sarmandal P, Grant JM. Effectiveness of ultrasound determination of fetal abdominal circumference and fetal ponderal index in the diagnosis of asymmetrical growth retardation. $\mathrm{Br}$ J Obstet Gynecol 1990; 97: $118-23$.

Schauseil-Zipf U, Hamm W, Stenzel B, Bolte A, Gladtke E. Severe intra-uterine growth retardation: obstetrical management and follow up studies in children born between 1970 and 1985. Eur J Obstet Gynecol Reprod Biol 1989; 30: 1-9.

Schneider KTM, Amberg-Wendland D, Renz S, Fürstenau U. Prospectiv randomisierte Untersuchung zum klinischen Wert der Dopplersonographie als Screeningverfahren. Gynäkol Rundsch 1991; 31: 139-40.

Schneider KT, Dumler EA, Lipperr A. Umfrage zur Verbreitung und Anwendung der Dopplersonographie im deutschsprachigen Raum. Geburtsh Frauenheilk 1993; 53: 56-60.

Schulman H, Winter D, Farmakides G, Ducey J, Guzman E, Coury A, Penny B. Pregnancy surveillance with Doppler velocimetry of uterine and umbilical arteries. Am J Obstet Gynecol 1989; 160: 192-6.

Scott A, Moar V, Ounsted M. Growth in the first four years. I. The relative effects of gender and weight for gestational age. Early Hum Dev 1982; 7: 17-28.

Secher NI, Kern Hansen P, Lenstrup C, Pedersen-Bjergaard L, Sindberg Eriksen P. Birthweight-for-gestational age charts based on early ultrasound estimation of gestational age. $\mathrm{Br}$ J Obster Gynaecol 1986a; 93: 128-34.

Secher NJ, Hansen PK, Lenstrup C, Eriksen PS. Controlled trial of ultrasound screening for light for gestational age (LGA) infants in late pregnancy. Eur J Obstet Gynecol Reprod Biol 1986b; 23: 307-13. 
Secher NJ, Lundbye-Christensen S, Qvist I, Bagger P. An evaluation of clinical estimation of fetal weight and symphysis fundal distance for detection of SGA infants. Eur J Obstet Gynecol Reprod Biol 1990; 38: 91-6.

Sijmons E. On screening for intrauterine growth retardation. Thesis, Utrecht, 1989a.

Sijmons EA, Reuwer PJHM, Beek E van, Bruinse HW. The validity of screening for small-for-gestational-age and low-weight-for-length infants by Doppler ultrasound. Br J Obstet Gynaecol 1989b; 96: 557-61.

Simon NV, O'Connor T], Shearer DM. Detection of intrauterine fetal growth retardation with abdominal circumference and estimated fetal weight using cross-sectional growth curves. J Clin Ultrasound 1990; 18: 685-90.

Soothill PW, Ajayi RA, Campbell S, Nicolaides KH. Prediction of morbidity in small and normally grown fetuses by fetal heart rate variability, biophysical profile score and umbilical artery Doppler studies. Br J Obstet Gynaecol 1993; 100: 742-5.

Spencer JA, Price J. Intraobserver variation in Doppler ultrasound indices of placental perfusion derived from different numbers of waveforms. J Ultrasound Med 1989; 8: 197-9.

Starfield B, Shapiro S, McCormick M, Bross D. Mortality and morbidity in infants with intrauterine growth retardation. J Pediatr 1982; 101: 978-83.

Stembera Z, Korvarfk J, Jungmannová C. Frequency of foetal growth deviations diagnosed by ultrasonic measurement and analysis of their causes. Acta Paediatr Scand, Suppl 1985; 319: 48-51.

Stuart B, Drumm J, FitzGerald DE, Duignan NM. Fetal blood velociry waveforms in normal pregnancy. Br J Obstet Gynaecol 1980; 87: 780-5.

Stuart JM, Healy TJ, Sutton M, Swingler GR. Symphysis-fundus measurements in screening for small-fordates infants: a community based study in Gloucestershire. J R Coll Gen Pract 1989; 39: 45-8.

Swyer PR. How small is too small? A personal opinion. Acta Pediatr 1992; 81: 443-5.

Tanner JM. Growth as a target-seeking function. In: F Falkner, JM Tanner (eds). Human growth. A comprehensive treatise. Plenum Press, 1986; vol. 1, chapter 9: 173-4.

Tenovuo A, Kero P, Piekkala P, Korvenranta H, Sillanpää M, Erkkola R. Growth of 519 small for gestational age infants during the first two years of life. Acta Paediatr Scand 1987; 76: 636-46.

Tenovuo A, Kero P, Piekkala P, Korvenranta H, Erkkola R. Fetal and neonatal mortality of small-for-gestational age infants. A 15-year study of 381 cases. Eur J Pediatr 1988; 147: 613-5.

Theron GB, Pattinson RC. Management of patients with poor symphysis pubis-fundus growth by Doppler flow velocimetry of the umbilical artery - an effective method to detect the fetus at risk. Int J Gynaecol Obstet 1992; 39: 93-8.

Touwen BCL. Ontwikkelingsgang en samenhang van het neurologisch onderzoek. De neurologische ontwikkeling van de zuigeling. Bohn, Scheltema en Holkema, 1984.

Trudinger BJ, Cook CM, Giles WB, Connelly A, Thompson RS. Umbilical artery flow velocity waveforms in high-risk pregnancy. Randomised controlled trial. The Lancet 1987; i: 188-90.

Trudinger BJ, Cook CM, Giles W/B, Ng S, Fong E, Connelly A, Wilcox W. Fetal umbilical artery velocity waveforms and subsequent neonatal outcome. $\mathrm{Br}_{\mathrm{r}}$ J Obstet Gynaecol 1991; 98: 378-84. 
Tyrrell SN, Lilford RJ, Macdonald HN, Nelson EJ, Porter J, Gupta JK. Randomized comparison of routine vs highly selective use of Doppler ultrasound and biophysical scoring to investigate high risk pregnancies. Br J Obstet Gynaecol 1990; 97: 909-16.

Versluys C. Keuze van voor Nederland bruikbare perinatale groeicurven. T Kindergeneesk 1980; 48: 158-67.

Villar J, Belizán JM. The timing factor in the pathophysiology of the intrauterine growth retardation syndrome. Obstet Gynecol Surv 1982a; 37: 499-506.

Villar J, Belizán JM, Spalding J, Klein RE. Postnatal growth of intrauterine growth retarded infants. Early Hum Dev 1982b; 6: 265-71.

Villar J, Belizán JM. The evaluation of the methods used in the diagnosis of intrauterine growth retardation. Obstet Gynecol Surv 1986; 41: 187-99.

Visser GH, Stigter RH, Bruinse HW. Management of the growth-retarded fetus. Eur J Obstet Gynecol Reprod Biol 1991; 42 Suppl: 73-8.

Voorhorst FJ, Puyenbroek JI, Robertson EA, Bezemer PD, Kurver PHJ. Verschillen de geboortegewichten van vroeger en nu? Ned Tijdschr Geneeskd 1990; 134: 998-1002.

Voss GBWE. Severity of illness and costs of medical care in patients with acute myocardial infarction. Thesis, Maastricht, 1993.

Vugt JMG van, Ruissen CJ, Hoogland HJ, Haan J de. A prospective study of the umbilical artery waveform in appropriate-for-date and growth-retarded fetuses. Gynaecol Obstet Invest 1988a; 23: 217-25.

Vugt JMG van, Ruissen CJ, Nienhuis SJ, Hoogland HJ, Haan J de. Comparison of blood velocity waveform indices recorded by Pulsed Doppler en CW Doppler in the umbilical artery. Journal of Clinical Ultrasound 1988b; 16: 573-6.

Waldenström U, Axelsson O, Nilsson S. Ultrasonic dating of pregnancies: effect on incidence of SGA diagnoses. A randomised controlled trial. Early Hum Dev 1992; 30: 75-9.

Wälli R, Stettler T, Largo RH, Fanconi A, Prader A. Gewicht, Länge und Kopfumfang neugeborener Kinder and ihre Abhängigkeit von mütterlichen und kindlichen Factoren. Normwerte für das intrauterine Wachstum. Helv paediatr Acta 1980; 35: 397-418.

Wallin A, Gyllensward A, Westin B. Symphysis-fundus measurement in prediction of fetal growth disturbances. Acta Obstet Gynecol Scand 1981; 60: 317-23.

Walther FJ, Ramaekers LHJ. Growth in early childhood of newborns affected by disproportionate intrauterine growth retardation. Acta Paediatr Scand 1982; 71: 651-6.

Wennergren $M$. Antenatal screening and risk factors for intrauterine growth retardation. Int J Technol Assess Health Care 1992; 8 Suppl 1: 147-51.

Westin B. Gravidogram and fetal growth. Acta Obstet Gynecol Scand 1977; 56: 273-6.

White M, Ritchie J. Psychological stressors in antepartum hospitalization: reports of pregnant women. Matern Child Nurs J 1984; 13: 47-56.

WHO. Public health aspects of low birth weight. Third report of the Expert Committee on Maternal and Child Health. World Health Organisation Techn Rep Ser 1961; 217: 3-16.

Wilcox AJ. Intrauterine growth retardation: beyond birthweight criteria. Early Hum Dev 1983; 8: 189-93. 
Wilcox MA, Johnson IR, Maynard PV, Smith SJ, Chilvers CED. The individualised birthweight ratio: a more logical outcome measure of pregnancy than birthweight alone. $\mathrm{Br} \mathrm{J}$ Obstet Gynaecol 1993; 100: 342-7.

Winick M. The fetal brain. In: Fetal growth retardation. FA van Assche (ed). Churchill Livingstone, 1981: 63-72.

Wladimiroff JW, Wijngaard JA van den, Degani S, Noordam MJ, Eyck J van, Tonge HM. Cerebral and umbilical arterial blood flow velocity waveforms in normal and growth-retarded pregnancies. Obstet Gynecol 1987; 69: 705-9.

Yudkin PL, Aboualfa M, Eyre JA, Redman CWG, Wilkinson AR. New birthweight and head circumference centiles for gestational ages 24-42. Early Hum Dev 1987; 15: 45-52. 


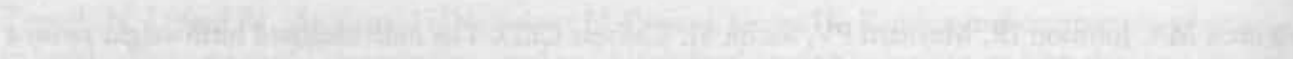

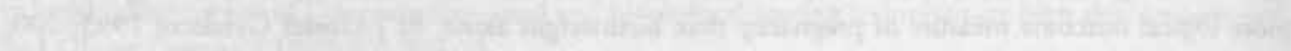

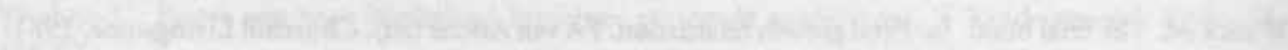

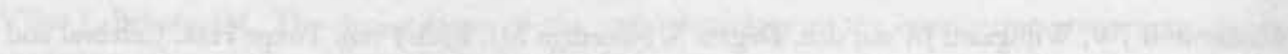

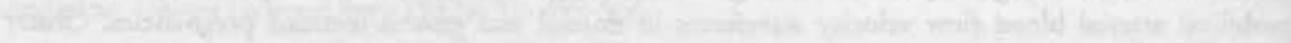

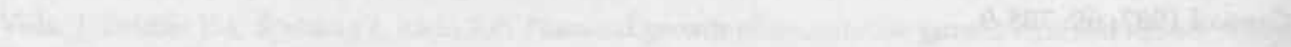

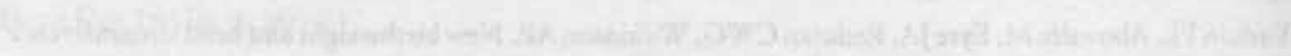

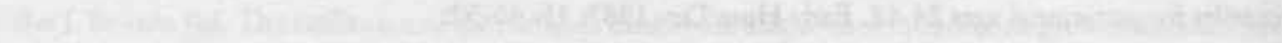

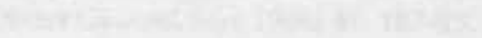




\section{Abbreviations}

\begin{tabular}{|c|c|}
\hline AGA & appropriate for gestational age \\
\hline AZM & Academisch Ziekenhuis Maastricht / University Hospital Maastricht \\
\hline $\mathrm{BPD}$ & biparietal diameter \\
\hline $\mathrm{CI}$ & confidence interval \\
\hline CRL & crown rump length \\
\hline CS & Caesarian section \\
\hline C-S & cross-sectional \\
\hline CTG & cardiotocogram \\
\hline $\mathrm{CW}$ & continuous wave \\
\hline DRG & diagnosis related groups \\
\hline GA & gestational age from 1st day of last menstrual period \\
\hline $\mathrm{H}$ & height \\
\hline $\mathrm{HC}$ & head circumference \\
\hline IUFD & intrauterine fetal death \\
\hline IUGR & intrauterine growth retardation \\
\hline LBW & low birth weight, birth weight less than 2500 grams \\
\hline LMP & last menstrual period \\
\hline LR & likelihood rario \\
\hline$N$ & size of the population \\
\hline n & number of participants or items \\
\hline OR & odds ratio \\
\hline$P$ & $\begin{array}{l}\text { centile, birth weight centile for the gestational age normalised for } \\
\text { parity and sex }\end{array}$ \\
\hline PD & pulsed Doppler \\
\hline PI & pulsatility index \\
\hline $\mathrm{PI}<\mathrm{M}+2 \mathrm{SD}$ & pulsatility index below two standard deviations above the mean \\
\hline Preterm & gestational age at birth less than 259 days \\
\hline Prev & prevalence \\
\hline $\mathrm{RCT}$ & randomised clinical trial \\
\hline $\mathrm{RI}$ & resistance index \\
\hline S/D & rario of systolic/diastolic frequencies (= A/B ratio) \\
\hline SD & standard deviation \\
\hline SDS & standard deviation score \\
\hline Sens & sensitivity \\
\hline SGA & small for gestational age \\
\hline sIUGR & suspected intrauterine growth retardation \\
\hline Spec & specificity \\
\hline Term & gestational age at birth between 259 and 294 days \\
\hline VSD & ventricular septal defect \\
\hline W & weight \\
\hline
\end{tabular}




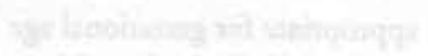

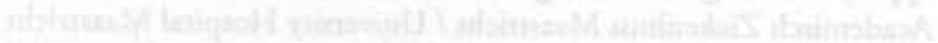

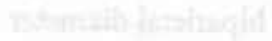

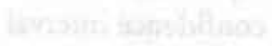

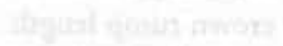

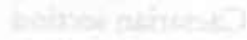

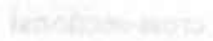

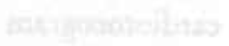

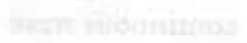

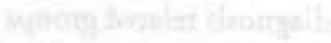

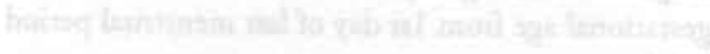

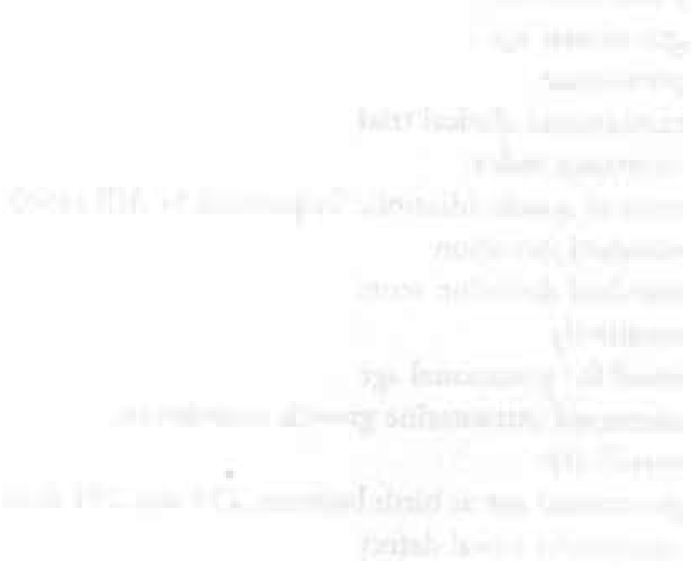




\section{Summary}

Chapter 1 outlines the incentive for the randomised clinical trial on Doppler ultrasound measurements as a reassurance test in suspected intrauterine growth retardation, presented in this thesis.

Intrauterine growth retardation (IUGR) is the condition whereby the fetus cannot fulfil its genetic growth potential. IUGR is a major clinical problem, as it is associated with increased perinatal morbidity and mortality. To reduce this perinatal risk, a pregnancy suspected of IUGR is often monitored in a clinical setting. Such a costly management policy is unnecessary if the fetus is genetically small.

Fetal growth is monitored in virtually the whole pregnant population by fundal height measurements. However, fundal height measurements cannot differentiate berween IUGR and genetic smallness. If IUGR is wrongly suspected, the patient and her direct environment will be burdened unnecessarily. Moreover, the financial consequences may be high.

For these reasons, an additional test which can differentiate IUGR from genetic smallness is of importance.

Chapter 2 reviews the literature on normal growth, fetal growth retardation and its consequences.

With regard to normal growth, a range of published anthropometric studies was reviewed. Plotting of the data in a single figure revealed a clear relation between age and the anthropometric parameters height, head circumference, and weight.

No conclusive definition of growth retardation could be obtained from the literature. A widespread standard is a birth weight below the 10th centile, corrected for the gestational age. In fact, such a threshold identifies infants who are small for gestational age (SGA). As a consequence of this definition, the perinatal risk is falsely assumed to be high in SGA due to genetic smallness. Cases with chromosomal and/or congenital defects differ in growth potential, and should therefore be excluded in the analysis of IUGR.

The underlying pathophysiological mechanisms of the aetiologic factors in IUGR, have not been fully elucidated. A major proportion of these factors leads to utero-placental haemodynamic insufficiency. An insufficient intrauterine environment is thought to affect fetal weight, height, and head circumference, in that order. This may lead to changes in fetal body proportions. After delivery, when the restrictive influence is 
discontinued, the infant will strive to catch-up to fulfil its genetic growth potential and regain its symmetrical proportions. The occurrence of such a catch-up growth could retrospectively be used as a standard for IUGR.

The quality of the utero-placental circulation can be assessed non-invasively with Doppler ultrasonography. However, the validity of Doppler ultrasound measurements in the detection of SGA infants is disappointing. The validity is somewhat higher than that of fundal height measurements and somewhat below the validity of ultrasonic fetal biometry. Whether perinatal outcome is improved by using Doppler ultrasound information in clinical decision making, is investigated in an extensive meta-analysis of published randomised clinical trials. Graphical presentation of each trial's results shows that detection of cases at risk by Doppler ultrasonography does not evidently improve outcome, in comparison with a control group.

On the other hand, several studies have shown that perinatal risk in suspected IUGR is low if the associated umbilical artery Doppler ultrasound results are in the normal range. This finding gives rise to the hypothesis that Doppler ultrasound measurements in the umbilical artery can be used as a reassurance test in suspected IUGR. Prevention of hospitalisation in cases identified as low risk by Doppler ultrasonography potentially saves costs, without this having a detrimental effect on perinatal outcome.

This dual hypothesis was investigated in the randomised clinical trial described in this thesis, by the Department of Obstetrics and Gynaecology, in collaboration with the Departments of Child Neurology and Paediatric Endocrinology of the University Hospital Maastricht, and the Departments of Health Economics and Methodology and Statistics of the University of Limburg.

Chapter 3 describes the study protocol of the present randomised clinical trial on Doppler ultrasonography as a reassurance test in suspected IUGR. One hundred and fifty patients with a singleton pregnancy in which intrauterine growth retardation of two or more weeks was suspected, were randomised over an intervention and a control group. In both groups, weekly Doppler ultrasound measurements were performed in the umbilical artery. The technique and background of umbilical artery Doppler sonography is discussed at length.

In the intervention group, the Doppler results were conveyed to the clinicians in charge, who were strongly requested not to hospitalise a patient for IUGR if the Doppler results were in the normal range. Doppler information was not available for clinical decision making in the control group, and routine clinical management for suspected IUGR was followed. The costs and effects of the Doppler based management in the intervention group were compared to the routine clinical management in the control group.

Costs were primarily approximated by using hospital admission rate and the number of hospital days. The net savings as a result of the Doppler based admission was estimated at approximately 15 million Dutch guilders annually in the Netherlands. 
In order to establish the effect of the study protocol, differences in obstetric management, outcome of pregnancy, neurological development, and postnatal growth between the two study groups were analysed. The occurrence of postnatal catch-up growth was used as a retrospective standard for IUGR. To analyse postnatal growth, a reference for optimal growth was constructed.

Chapter 4 presents the main results of the trial. The objective of the analysis was to test the differences in costs and outcome between the intervention and the control group. Stratification and randomisation led to two study groups which were comparable for most prognostic factors. The distribution of the Doppler ultrasound results was also similar in both groups.

The main objective of the trial was to reduce unnecessary hospitalisation for suspected IUGR during pregnancy, without detrimental effects on perinatal outcome. Contrary to expectations, hospitalisation rate did not differ significantly between the study groups. Of influence on this finding was that 14 participants in the intervention group were hospitalised for suspected IUGR despite their normal Doppler results. As this clinical management was not in accordance with the study protocol, these cases were by definition protocol deviations. An additional effect was that although the obtained hospitalisation rate during pregnancy in the intervention group coincided with the expected rate, the rate in the control group was far lower than expected from a questionnaire among obstetricians in the Dutch university hospitals (chapter 7). The duration of hospital admission was significantly lower in the intervention group, than that in the control group.

The use of Doppler ultrasound measurements as reassurance test in suspected IUGR was not shown to have a detrimental effect on the outcome. Obstetric intervention, gestational age at delivery, birth weight or centile did not differ between the two study groups. Neither did neonatal hospital admission rate or duration of admission.

Neurological development of infants after delivery and at six and nine months was comparable in the intervention group and the control group. Catch-up growth in the first six months after delivery occurred in a similar rate in both study groups.

In the secondary analysis presented in chapter 5, some notable relations were found between the Doppler ultrasound results and the outcome of the trial. These relations had not been defined beforehand as objective of the trial.

If the Doppler ultrasound results were in the normal range, hospitalisation rate was notably lower in both study groups. Likewise, raised Doppler results were associated with a high admission rate. This suggests that risk assessment by umbilical artery Doppler ultrasonography is comparable to that of routine outpatient checkups.

The probability of an uneventful outcome of pregnancy was higher if the Doppler results were normal, both in the intervention and in the control group. Further analysis of the 
protocol deviations tentatively indicated that these fetuses were small, but possibly not at high risk.

A significant relation between the results of repeated neurological assessments and the Doppler findings, was only found at the neurological examination after delivery. However, neonates with raised Doppler results during pregnancy, were significantly lighter and shorter than neonates with normal Doppler findings. It is probable that the appearance of the neonates examined could have biased the neurological examination after delivery.

Six months after delivery, the difference in weight was no longer significant between the Doppler subgroups. Infants who had had raised Doppler values during pregnancy had caught-up with their normal Doppler peers.

Chapter 6 discusses the results of the randomised clinical trial. The differences between the hospitalisation rate expected on the basis of the questionnaire and the results obtained in the trial, may be partly explained by a more critical attitude of the clinicians in charge towards the indications for admission, due to the implementation of the study protocol. This could have reduced hospitalisation during pregnancy in the control group. On the other hand, patients with suspected IUGR in the intervention group were hospitalised because of associated risk factors, in spite of their normal Doppler results. Using the results of the trial, the implementation of repeated umbilical artery Doppler ultrasound assessments in the umbilical artery as reassurance test in suspected IUGR could lead to a net saving of almost 39 million Dutch guilders on a national level per year.

Perinatal death is an important outcome measure in perinatal studies. After exclusion of cases with chromosomal and/or congenital defects, intrauterine fetal death occurred once in the intervention and twice in the control group. In two cases, this lethal outcome may not have been inevitable. In the intervention group case, the decision was made in consultation with the parents, to abstain from obstetrical intervention because of the estimated poor postnatal chances. In one case in the control group, the IUFD might have been prevented if the Doppler information had been available for clinical decision making. However, these numbers are too small to draw further conclusions from.

Notable was that the finding of a not normal final result at a neurological development examination occurred more frequently at six months than after delivery or at nine months. This is in agreement with findings in the literature. These transient abnormal neurological findings are associated with problems at school age.

Cases associated with raised Doppler values had markedly lower mean weights and marginally smaller mean heights at birth than their normal Doppler peers. That the difference in mean head circumference was not significantly different between the Doppler subgroups, supports the theory of a brain sparing effect. The occurrence of a postnatal catch-up growth, which resulted in a not significant difference in weight six months after delivery, could retrospectively be used to identify IUGR. 
Chapter 7 presents the questionnaire on the prevalence of suspected IUGR and the content of its routine clinical management among obstetricians in the university hospitals in the Netherlands. The findings were used in the planning of the study protocol of the trial presented in this thesis.

Fetal size was monitored by fundal height measurements in $75 \%$ of the obstetricians questioned. The prevalence of suspected IUGR was estimated at approximately $12 \%$ of the singleton pregnancies at the university hospital outpatient departments. In the opinion of the respondents, almost $70 \%$ of these cases was admitted to hospital for IUGR.

According to the questionnaire, Doppler ultrasound information was used in clinical decision making in five out of eight university hospitals in the Netherlands. 


\section{Samenvatting}

In hoofdstuk 1 wordt de achtergrond gepresentecd van het in dit procfschrift beschreven gerandomiseerde klinische onderzoek naar Doppler ultrageluidsmetingen als geruststellingstest bij verdenking op intra-uteriene groeiretardatie.

Als de foetus zijn genetische groeipotentie niet kan vervullen, is er sprake van intra-uteriene groeiretardatie (IUGR). IUGR is een belangrijk klinisch probleem, omdat her gepaard gaat met een verhoogde perinatale morbiditeit en mortaliteit. $O \mathrm{~m}$ dit perinatale risico te verminderen, vindt er bij verdenking op IUGR vaak klinische foetale bewaking plaats. Als er sprake is van genetisch klein-zijn, is een dergelijk kostbaar beleid echter onnodig.

De foetale groei wordt voor vrijwel de gehele zwangere populatie gecontrolecrd door middel van metingen van de fundushoogte. Met deze metingen kan echter niet worden gedifferentieerd tussen IUGR en genetisch klein-zijn. Een vals positieve verdenking op IUGR belast de moeder en haar directe omgeving onnodig en de financiële consequenties kunnen aanzienlijk zijn. Daarom is een aanvullende test die een onderscheid maakt tussen IUGR en genetisch klein-zijn van groot belang.

In boofdstuk 2 wordt de literatuur over normale groei, intra-uteriene groei retardatic, en de gevolgen van IUGR besproken.

Er wordt een overzicht gegeven van gepubliceerde anthropometrische studies over normale groei. Het plotten van de data in één figuur laat een duidelijke relatie zien tussen de leeftijd en de anthropometrische parameters lengte, hoofdomtrek, en gewicht.

Uit de literatuur kan geen eenduidige definitie van IUGR worden verkregen. In de meest gangbare definitie wordt een neonaat als te klein beschouwd wanneer het geboortegewicht onder het $10 \mathrm{e}$ percentiel voor de zwangerschapsduur ligt. In feite identificeert cen dergelijk afkappunt small for gestational age (SGA) neonaten. Ten gevolge van deze definitie wordt bij SGA ten gevolge van genetisch klein-zijn, ten onrechte een hoog-risico verondersteld. Bij congenitale en/of chromosomale afwijkingen is er sprake van een andere groeipotentie. Deze gevallen moeten daarom worden uitgesloten bij een analyse van IUGR.

Van veel factoren die aan IUGR ten grondslag liggen, is het onderliggend mechanisme niet exact bekend. Een belangrijk deel van deze factoren wordt geassocieerd met een deficiënte utero-placentaire circulatie. Een insufficiënt intra-uterien milieu lijkt respectievelijk het foetale gewicht, de lengte, en de hoofdomtrek in afnemende mate te 
beïnvloeden. Dit kan leiden tot veranderingen in de foetale lichaamsproporties. Als de groeibeperking na de geboorte is opgeheven, zal de neonaat zijn groeiachterstand proberen in te halen, om zo zijn groeipotentie te vervullen en de symmetrie van de lichaamsproporties te herstellen. Het optreden van een dergelijke inhaalgroei zou kunnen worden gebruikt als retrospectieve standaard voor IUGR.

Met behulp van Doppler ultrasonografie kan de kwaliteit van de utero-placentaire circulatie non-invasief worden gemeten. De validiteit waarmee SGA door Doppler ultrageluidsmetingen wordt geïdentificeerd, is echter teleurstellend. De validiteit van Doppler ultrageluidsmetingen is iets groter dan die van metingen van de fundushoogte en iets kleiner dan die van echoscopische foetale biometrie. Of het gebruik van Doppler ultrageluidsmetingen een positief effect heeft op de perinatale uitkomst, werd onderzocht in een uitgebreide meta-analyse van gepubliceerde gerandomiseerde klinische trials. De grafische weergave van de resultaten van de onderzoeken laat zien dat, in vergelijking met een controlegroep, de uitkomsten niet evident worden verbeterd als de risico gevallen met Doppler ultrasonografie worden geïdentificeerd.

Anderzijds hebben een aantal onderzoeken aangetoond dat de van groeiretardatie verdachte foetus geen verhoogd risico lijkt te hebben als de Doppler ultrageluidsbevindingen in de arteria umbilicalis binnen het normale bereik liggen. Deze bevinding heeft geleid tot de hypothese dat Doppler ultrageluidsmetingen in de arteria umbilicalis kunnen worden gebruikt als geruststellingstest bij verdenking op IUGR. Het voorkómen van ziekenhuisopname in die gevallen waarbij de Doppler bevindingen op een laag risico duiden, leidt potentieel tot vermindering van kosten, zonder dat dit een negatief effect heeft op de perinatale uitkomst.

Deze tweeledige hypothese werd onderzocht door middel van een gerandomiseerde klinische trial door de vakgroep Gynaecologie/Obstetrie, in samenwerking met de vakgroepen Kinderneurologie en Kinder-endocrinologie van het Academisch Ziekenhuis Maastricht, en de vakgroepen Economie van de Gezondheidszorg en Methodologie en Statistiek van de Rijksuniversiteit Limburg.

In hoofdstuk 3 wordt de methodiek beschreven van de gerandomiseerde klinische trial (RCT) betreffende Doppler ultrageluid als geruststellingstest bij verdenking op intrauteriene groeiachterstand. Honderdvijftig zwangeren met een eenling zwangerschap met een klinische verdenking op IUGR werden willekeurig verdeeld over een interventiegroep en een controlegroep. In beide groepen werden wekelijks Doppler metingen in de arteria umbilicalis verricht. In de interventiegroep werd de uitslag ter beschikking gesteld van de behandelaar, die met klem verzocht werd een zwangere niet op te nemen voor groeiachterstand als de Doppler uitslag normaal was. In de controlegroep werden de Doppler uitslagen niet bekend gemaakt en werd het huidige routine beleid voor groeiretardatie gevolgd.

De verschillen in kosten en effecten tusen het op Doppler ultrageluid gebaseerde beleid in de interventiegroep en het routine beleid in de controle groep werden bepaald. 
De kosten werden in eerste instantie benaderd aan de hand van opnamepercentage en -dagen. De netto besparing die zou kunnen worden gerealiseerd met behulp van het op Doppler gebaseerde opnamebeleid, werd geschat op ongeveer 15 miljoen gulden per jaar in Nederland.

Om de effecten van het protocol te analyseren, werden de verschillen tussen de twee studiegroepen in obstetrisch beleid, uitkomst van de zwangerschap, postnatale neurologische ontwikkeling, en postnatale groei bepaald. Het vóórkomen van postnatale inhaalgroei werd geïntroduceerd als potentiële retrospectieve standaard voor IUGR. Om postnatale groei te kunnen analyseren, werd een referentie voor optimale groei geconstrueerd.

In hoofdstuk 4 worden de resultaten van de RCT gepresenteerd. Het doel van de analyse was om kosten en effecten in de interventiegroep en de controlegroep te vergelijken. Stratificatie en randomisatie leidde tot twee studiegroepen die vergelijkbaar waren voor de meeste prognostische factoren. De verdeling van de Doppler resultaten was ook vergelijkbaar.

Het hoofddoel van het onderzoek was om bij verdenking op IUGR onnodige ziekenhuisopname tijdens de zwangerschap te voorkomen, zonder dat dit beleid nadelige effecten had op de perinatale uitkomst. In tegenstelling tot wat er volgens de hypothese werd verwacht, verschilde het percentage ziekenhuisopnames niet significant tussen de studiegroepen. Hierbij waren twee factoren opmerkelijk. Veertien participanten uit de interventiegroep werden, ondanks hun normale Doppler resultaten, op verdenking van groeiachterstand opgenomen. Daar dit beleid niet conform het protocol was, zijn dit per definitie protocol deviaties. Daarnaast was het opnamepercentage in de controlegroep lager dan vooraf was geschat op grond van een enquête onder obstetrici werkzaam in de academische ziekenhuizen in Nederland (hoofdstuk 7). In de interventiegroep kwam het verkregen opnamepercentage tijdens de zwangerschap wel overeen met het uit de enquête verwachte opnamepercentage. De opnameduur tijdens de zwangerschap bleek in de interventiegroep significant korter dan in de controlegroep.

Het gebruik van Doppler ultrageluidsmetingen als geruststellingstest bij verdenking op groeiachterstand bleek geen negatief effect te hebben op de uitkomst van de zwangerschap. De twee studiegroepen waren vergelijkbaar met betrekking tot het vóorkomen van kunstverlossingen, zwangerschapsduur bij de bevalling en in geboortegewichten en -percentielen. Het percentage neonaten dat werd opgenomen en de neonatale opnameduur waren ook niet significant verschillend.

De neurologische ontwikkeling van de kinderen na de geboorte en op de leeftijd van zes en negen maanden was vergelijkbaar in de twee studiegroepen. Inhaalgroei in de eerste zes maanden na de geboorte kwam even vaak voor in de interventie- als in de controlegroep. 
In hoofdstuk 5 wordt een secundaire analyse gepresenteerd, waarin enkele opmerkelijke relaties werden gevonden tussen de Doppler uitslagen en de studieresultaten. Deze analyse was niet vooraf gedefinieerd als hoofddoel van het onderzoek.

Als de Doppler ultrageluidsbevindingen in het normale bereik lagen, was de kans op opname tijdens de zwangerschap duidelijk lager in beide studiegroepen. Verhoogde Doppler waarden waren gerelateerd aan een hoge kans op opname. Dit suggereert dat de risicobepaling door middel van Doppler ultrasonografie in de arteria umbilicalis vergelijkbaar is met die van routine zwangerschapscontroles.

Zowel in de interventiegroep als in de controlegroep was de kans op een ongestoorde uitkomst van de zwangerschap hoger als de Doppler waarden normaal waren. Uit verdere analyse van de protocol deviaties bleek dat, hoewel deze foetussen klein waren, het perinataal risico niet evident was verhoogd.

Een significante relatie tussen de resultaten van de herhaalde neurologische onderzoeken en de Doppler bevindingen, werd alleen gevonden bij het neurologisch onderzoek na de geboorte. Neonaten met verhoogde Doppler waarden gedurende de zwangerschap, waren echter significant lichter en korter bij de geboorte dan neonaten waarbij de Doppler normaal was. Daardoor is het mogelijk dat het uiterlijk van de neonaten het neurologisch onderzoek na de geboorte beïnvloedde.

Zes maanden na de geboorte was het verschil in gewicht tussen de Doppler subgroepen niet meer significant. De kinderen met een verhoogde Doppler hadden hun achterstand ten opzichte van die met een normale Doppler ingehaald. Het optreden van een dergelijke inhaalgroei na de geboorte zou een retrospectief bewijs kunnen zijn voor IUGR.

In hoofdstuk 6 worden de belangrijkste resultaten van de RCT verder besproken. Het verschil tussen de verwachte ziekenhuisopname op grond van de enquête en de verkregen opnameresultaten van de trial, kan deels worden verklaard door een meer doordachte indicatiestelling voor ziekenhuisopname. Het opnamepercentage in de controlegroep was lager dan verwacht, mogelijk doordat de betrokken clinici zich er van bewust waren dat hun opnamegedrag werd bestudeerd. Het opnamepercentage in de interventiegroep werd negatief beïnvloed doordat patiënten, waarbij er een verdenking was op intrauteriene groeiachterstand, ondanks een geruststellende Doppler uitslag werden opgenomen vanwege andere risicofactoren.

Op grond van de onderzoeksgegevens kan worden geconcludeerd dat de toepassing van Doppler ultrageluid als geruststellingstest bij verdenking op IUGR, op nationaal niveau, een jaarlijkse besparing van bijna 39 miljoen gulden op zou kunnen leveren.

Een belangrijke effectmaat in perinataal onderzoek is de perinatale sterfte. Intra-uteriene vruchtdood (IUVD) kwam eenmaal voor in de interventiegroep en tweemaal in de controlegroep, na exclusie van gevallen met chromosomale en/of congenitale afwijkingen. Bij twee foetussen had deze fatale uirkomst wellicht voorkomen kunnen worden. $\mathrm{Bij}$ de IUVD in de interventiegroep werd in overleg met de ouders besloten om van 
obstetrische interventie af te zien vanwege de als matig ingeschatte postnatale kansen. Bij één IUVD in de controlegroep had de sterfte wellicht kunnen worden voorkomen als de Doppler informatie beschikbaar was geweest. Deze getallen zijn echter te klein om er vergaande conclusies uit te kunnen trekken.

Opmerkelijk was dat afwijkende bevindingen bij het neurologisch onderzoek meer voorkwamen bij zes maanden dan bij het onderzoek na de geboorte of bij negen maanden. Dit komt overeen met resultaten in de literatuur. Dergelijke voorbijgaande afwijkende neurologische bevindingen worden wel geassocieerd met problemen op de schoolleeftijd.

De neonaten waarbij de Doppler waarden verhoogd waren tijdens de zwangerschap, waren evident lichter en marginaal korter bij de geboorte dan neonaten waarbij de Doppler resultaten normaal waren. Dat de gemiddelde hoofdomtrekken niet significant verschilden tussen de Doppler subgroepen, ondersteunt de theorie van het brain sparing effect. Het vóórkomen van inhaalgroei, waardoor het verschil in gewicht tussen de Doppler subgroepen met zes maanden niet langer significant was, werd gebruikt als retrospectieve standaard voor IUGR.

Hoofdstuk 7 presenteert een enquête over het vóórkomen van, en het beleid bij verdenking op IUGR onder obstetrici werkzaam in de academische ziekenhuizen in Nederland. De gegevens uit deze enquête werden gebruikt bij de planning van het studieprotocol van het in dit proefschrift beschreven onderzoek.

De foetale grootte werd door $75 \%$ van de responderende obstetrici gecontroleerd door middel van metingen van de fundushoogte. De prevalentie van verdenking op IUGR werd geschat op ongeveer $12 \%$ van de eenling zwangerschappen op de polikliniek van de academische ziekenhuizen. $\mathrm{Bijna} 70 \%$ van deze gevallen werd, volgens de respondenten, in het ziekenhuis opgenomen.

Uit de enquête bleek tevens dat Doppler ultrageluidsinformatic in vijf van de acht academische ziekenhuizen werd gebruikt bij het bepalen van het obstetrisch beleid. 


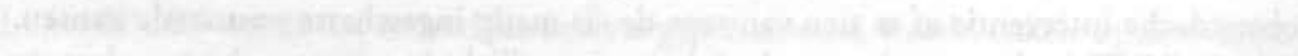

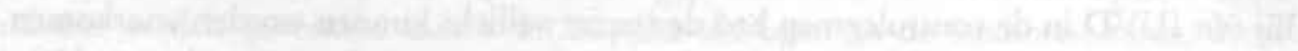

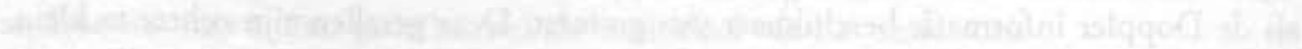

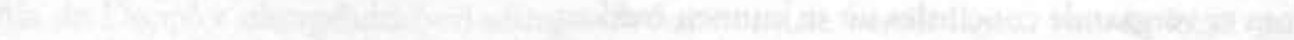

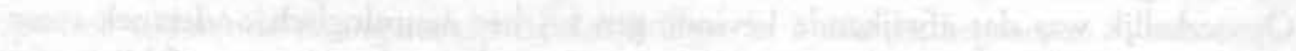

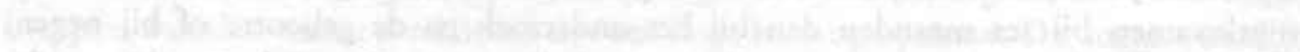

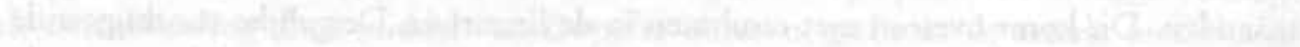

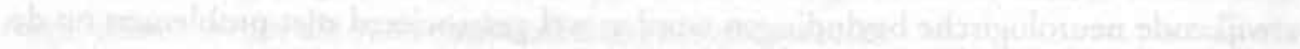

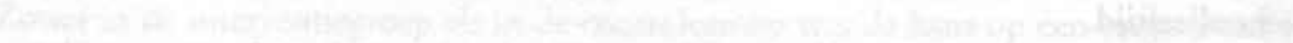

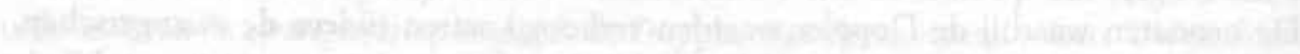

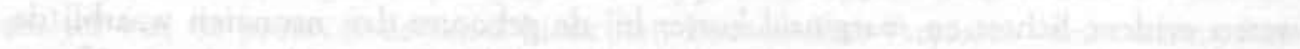

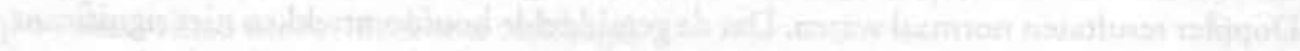

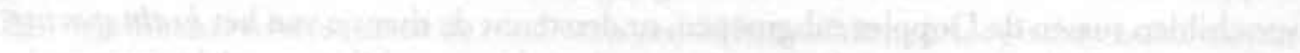

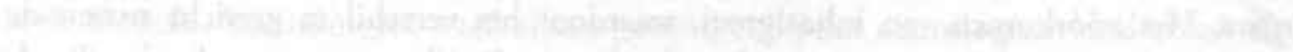

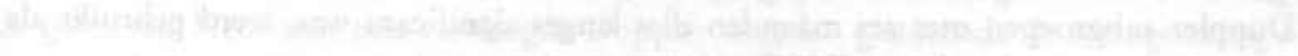

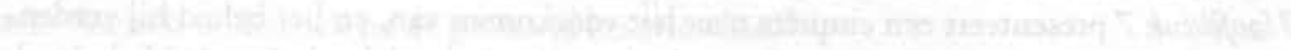

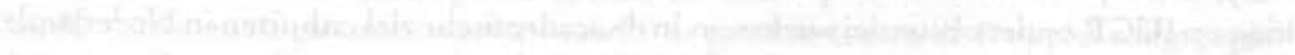

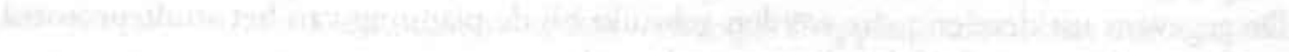

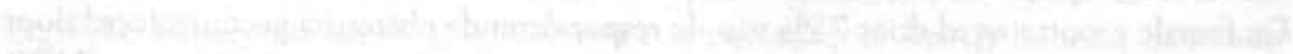

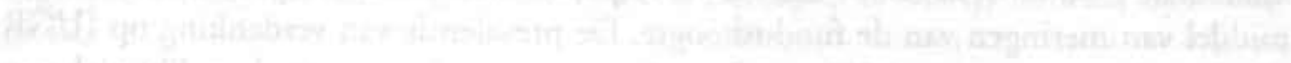

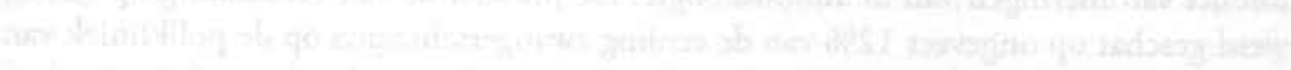

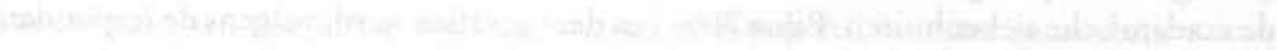

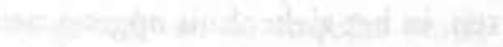

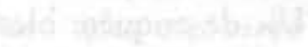
$x^{4}=0$ 


\section{Appendix 1}

\section{Neurological examination of neonates at term according to Prechtl (Prechtl 1977)}

The observation period state

resting posture

spontaneous motor activity

athetoid postures and movements

tremor

other movements

skin

respiration

Examination in the supine position skull

face

lip reflex

jaw jerk (masseter) reflex

glabella reflex

posture

spontaneous motor activity

Moro response

crying

The eyes

observation of the eyes

pupils

reaction of pupils to light

optical blink reflex

acoustic blink reflex

supine suspension

doll's-eye test

asymmetrical tonic neck reflex

Power and passive movements resistance against passive movements power of active movements

range of movement

recoil of the forearm at the elbow

muscular consistency

Reflexes and responses

biceps reflex

knee jerk

ankle clonus

palmar grasp

plantar grasp

Babinski reflex

magnet response

withdrawal reflex

rooting response

sucking response

traction test

control of head movements during the

sitting posture

Moro response

In the prone position

inspection and palpation of the vertebral column

spontaneous movements of the head spontaneous crawling and Bauer's

response

the ankle jerk

incurvation of the trunk (Galant's

response)

posture of the head and limbs in prone suspension 


\section{Appendix 2}

\section{Neurological examination according to Touwen at the corrected age of six and nine months (Touwen 1984)}

Anamnestic assessment of development

- general impression by the parents

- developmental stadia

- occurrence of paroxysmal events

- asymmetrical reactions at manipulations

- hand preference

- position preference

- rate of development in comparison with other children

\section{Observation}

- state of consciousness

- spontaneous position of the arms

- spontaneous position of the legs

- spontaneous motor activity of the arms

- spontaneous motor activity of the legs

- spontaneous motor activity of the legs in upright suspension

- purposeful motor activity of arms and legs

- voluntary grasping

- coordination of the arms

- position of the head, trunk and arms in prone position

- forward movement in prone position

- rolling over from supine to prone position
- rolling over from prone to supine position

- spontaneous lifting of the head in supine position

- can achieve sitting position

- duration of sitting position

- position of the trunk in sitting position

- can pull up to standing position

\section{Reactions and responses}

- palmar grasp reflex

- reaction to a push against the shoulder in sitting position

- following an object with the eyes and rotation of the head and trunk in sirting position

- optical placing reaction of the hands if moved towards a surface in prone suspension

- parachute reaction of arms and hands at being held up in prone position

- optical placing reaction of the feet if moved towards a surface in vertical suspension

- foot sole reflex

- acoustic orientation

- Moro reaction 


\section{Appendix 3}

\section{Anthropometric reference curves}

The table shows the formulas for the mean, and for the value of one standard deviation (1SD), of the constructed reference curves for height, head circumference (HC), and weight. For each anthropometric parameter, the values for $1 S D$ were, respectively, added and subtracted from the curve fit of the mean to obtain the constructed reference curves shown in figures 3.8-3.10. Separate reference curves were constructed for boys and girls. As discussed in section 3.3.4, the formulas were used to calculate standard deviation scores (SDS) for the anthropometric measurements of the infants in the trial. The method by which these curve fits were achieved, was described in this section.

The formulas of the anthropometric parameters had the following general function:

from conception to 280 days: $y=a+b \mathrm{x}$

from 280 to 640 days: $y=c+d x+e x^{2}+f x^{3}$

whereby:

$x$ is age in days; $y$ is the mean (or the value of $1 S D$ as indicated) of the anthropometric parameter at that age; The values for the variables $a$ to $f$ are shown in the table. 


\begin{tabular}{|c|c|c|c|c|c|c|c|c|c|c|}
\hline & \multirow[t]{2}{*}{ Sex } & \multirow[t]{2}{*}{ Fit } & \multicolumn{3}{|c|}{ Age 14 to 280 days } & \multicolumn{5}{|c|}{ Age 280 to 640 days } \\
\hline & & & a & b & c & $d$ & c & & $\mathrm{f}$ & $a^{2}$ \\
\hline Height $(\mathrm{cm})$ & $\begin{array}{l}\text { boys } \\
\text { girls }\end{array}$ & $\begin{array}{l}\text { mean } \\
1 S D \\
\text { mean } \\
1 S D\end{array}$ & $\begin{array}{l}-2.67 \\
-0.114 \\
-2.65 \\
-0.102\end{array}$ & $\begin{array}{l}0.191 \\
0.00821 \\
0.189 \\
0.00725\end{array}$ & $\begin{array}{c}-14.4 \\
4.27 \\
-8.32 \\
0.851\end{array}$ & $\begin{array}{c}0.350 \\
-0.0150 \\
0.314 \\
0.00544\end{array}$ & $\begin{array}{c}-4.90 \\
0.334 \\
-4.36 \\
-0.0673\end{array}$ & $\begin{array}{l}\times 10^{-4} \\
\times 10^{-4} \\
\times 10^{-4} \\
3 \times 10^{-4}\end{array}$ & $\begin{array}{l}2.57 \\
-0.222 \\
2.33 \\
0.0373\end{array}$ & $\begin{array}{l}\times 10^{-7} \\
\times 10^{-7} \\
\times 10^{-7} \\
\times 10^{-7}\end{array}$ \\
\hline $\mathrm{HC}(\mathrm{cm})$ & $\begin{array}{l}\text { boys } \\
\text { girls }\end{array}$ & $\begin{array}{l}\text { mean } \\
1 S D \\
\text { mean } \\
1 S D\end{array}$ & $\begin{array}{l}-1.85 \\
-0.0625 \\
-1.82 \\
-0.0634\end{array}$ & $\begin{array}{l}0.132 \\
0.00447 \\
0.130 \\
0.00453\end{array}$ & $\begin{array}{c}-9.99 \\
0.417 \\
-6.63 \\
2.58\end{array}$ & $\begin{array}{l}0.260 \\
0.00519 \\
0.237 \\
-0.00902\end{array}$ & $\begin{array}{l}-4.21 \\
-0.108 \\
-3.78 \\
0.177\end{array}$ & $\begin{array}{l}\times 10^{-4} \\
\times 10^{-4} \\
\times 10^{-4} \\
\times 10^{-4}\end{array}$ & $\begin{array}{l}2.39 \\
0.0750 \\
2.14 \\
-0.108\end{array}$ & $\begin{array}{l}\times 10^{-7} \\
\times 10^{-7} \\
\times 10^{-7} \\
\times 10^{-7}\end{array}$ \\
\hline Weight $(\mathrm{kg})$ & $\begin{array}{l}\text { boys } \\
\text { girls }\end{array}$ & $\begin{array}{l}\text { mean } \\
\text { ISD } \\
\text { mean } \\
\text { ISD }\end{array}$ & $\begin{array}{l}-4.26 \\
-0.143 \\
-4.10 \\
-0.0545\end{array}$ & $\begin{array}{l}0.0269 \\
0.00218 \\
0.0260 \\
0.00166\end{array}$ & $\begin{array}{l}-12.1 \\
-1.20 \\
-10.0 \\
-0.162\end{array}$ & $\begin{array}{l}0.0818 \\
0.00937 \\
0.0679 \\
0.00237\end{array}$ & $\begin{array}{l}-1.13 \\
-0.146 \\
-0.860 \\
-0.0130\end{array}$ & $\begin{array}{l}\times 10^{-4} \\
\times 10^{-4} \\
\times 10^{-4} \\
0 \times 10^{-4}\end{array}$ & $\begin{array}{l}0.608 \\
0.0857 \\
0.429 \\
0.00430\end{array}$ & $\begin{array}{l}\times 10^{-7} \\
\times 10^{-7} \\
\times 10^{-7} \\
\times 10^{-7}\end{array}$ \\
\hline
\end{tabular}




\section{Acknowledgements}

Er zijn veel mensen die het verdienen bedankt te worden. Ik wil iedereen bedanken die tijd nam om vragen te beantwoorden, dingen voor me op te zoeken, of in te springen bij de organisatie. Een aantal mensen wil ik met name noemen.

Dr H.J. Hoogland, beste Henk. Als co-promotor hield je steeds de grote lijn in de gaten. Als ik aan details ten onder dreigde te gaan, wees je me opnieuw de goede richting. Nooit heb ik tevergeefs een beroep op je gedaan.

Prof. dr J. de Haan. Met vaderlijke gestrengheid heeft u een einde gemaakt aan mijn dralen, waardoor het nu dan toch af is. Bedankt.

Dr C.J. Ruissen, beste Kees. Als maatje, klaagmuur, en redder in de nood heb je me de basis bijgebracht van de fysica van ultrageluid en me meer dan wegwijs gemaakt op de computer.

Dr J.H.S. Vles, beste Hans. Ik heb veel geleerd van je scherpe observaties tijdens de honderden neurologische onderzoeken van de kinderen in de studie. Je betrokkenheid bij je patiëntjes is indrukwekkend.

Dr W.J.M. Gerver, beste Willem-Jan. Jouw enthousiasme over de anthropometrie was aanstekelijk. Angèle Gerver, bedankt voor de hulp bij de metingen.

Dr A. Ament, beste André. Je stap voor stap analyse van mijn protocol was verhelderend. Ik zal met interesse de verdere ontwikkeling van de analysemethode volgen.

Dr L. Volovics, beste Lex. Bedankt voor de vele uren waarin we hebben zitten puzzelen over de groeicurves.

Dr H. Schouten, beste Hubert. Bedankt dat je steeds bereid was om vragen over de statistiek te beantwoorden.

Zonder de verwijzing van patiënten door de gynaecologen, arts-assistenten, en verloskundigen van de afdeling Gynaecologie/Obstetrie van het Academisch Ziekenhuis Maastricht, zou het onderzoek nooit tot stand zijn gekomen.

De medewerkers van de poliklinieken Gynaecologie/Obstetrie, Kindergeneeskunde, en Neurologie hebben mij zeer geholpen bij het organiseren van de vele afspraken.

Van het secretariaat Gynaecologie/Obstetrie wil ik met name bedanken Manon Gordijn (what would I be without you?), Monique Jansen, de vraagbaak van de afdeling, en Cecile Gorissen.

Mevrouw T. Ausems, hoofd Consultatiebureau Artsen Heuvelland, en de medewerkers van de Consultatiebureaus bedankt voor de hulp bij het verzamelen van de groeigegevens van de deelnemertjes. 
Bedankt Marlie Kessels, Ingrid Rabelink, Jean-Paul Kleijne, en Nicole Zusterzeel voor jullie hulp tijdens student assistentschappen danwel wetenschapsstages.

Bedankt onderzoeksmaatjes van het vrijdagmiddag AIO-clubje voor het constructieve commentaar en de mogelijkheid tot afreageren. Bedankt Ineke van Lijnschoten, steeds als ik dacht dat het goed was, had jij nog uitgebreid commentaar.

Prof. dr H.A.J. Struyker Boudier, beste Harry. De aanzet tot mijn wetenschappelijke interesse werd gegeven tijdens de vele discussies en het vermaledijde verplichte wetenschapsblok. Bedankt.

Dr J.M.G. van Vugt, beste John. Met het Canisius als gemeenschappelijke achtergrond klikte het meteen. Bedankt voor het begeleiden van mijn eerste schreden in het Doppler-onderzoek.

Ir R.J. Nienhuis, lieve paps. Met het maken van de plaatjes heb je mij een reuze klus bespaard. Ons beider perfectionisme makkte het niet makkelijk. I $\mathrm{k}$ ben zeer tevreden. Ir K.J. Blok van Laer, beste Karel. Mijn hele bestaan al ben je uiterst kritisch betrokken bij mijn activiteiten. Door het vermogen echt te luisteren, hebben jij en Mieke een zeer bijzondere plaats in mijn leven. Je was onvermoeibaar in het streven naar correctheid in het manuscript, volgens de Philips standaard. Voor mij was je, als heer van stand, vanaf het begin de aangewezen persoon voor het paranimfschap, als je begrijpt wat ik bedoel. Datawyse, Ruud Leliveld. Bedankt dat je in deze spannende fase steeds koelbloedig een oplossing wist te vinden voor alle (lay-out) problemen.

André Casteleyn, het basis-idee van het onderzoek is door jou op perfecte wijze vormgegeven in het omslag.

Familie en vrienden. Bedankt dat jullie steeds bereid waren er begrip voor op te brengen als ik weer eens aan mijn proefschrift moest werken. We hebben elkaar de afgelopen jaren minder gezien dan we hadden gewild.

Pieter, we hebben de afgelopen jaren vaak gediscussieerd over het onderzoek. Als ik het wel eens niet zag zitten, kon jij het geheel relativeren en me de postitieve kanten laten zien. Tijdens de schrijffase kon jij uirstekend overzien of datgene wat ik probeerde over te brengen, ook op papier overkwam. Je adviezen over de stijl hebben de leesbaarheid van het proefschrift zeer bevorderd. En verder had ik het zonder jou gewoon niet gered. 


\section{Curriculum vitae}

1963 geboren te Nijmegen op 15 februari

1973 - 1977 primary en highschool, Engeland

1982 eindexamen atheneum-B, Canisius College, Nijmegen

1982 - 1983 studie kunstgeschiedenis: Christie's Fine Arts Course, Londen

1983 aanvang studie geneeskunde, Rijksuniversiteit Limburg, Maastricht

1986 - 1987 student assistent, vakgroep Gynaecologie en Obstetrie, Academisch Ziekenhuis Maastricht

1988 onderzoeksassistent, Department of Gynaecologic and Obstetric Ultrasound, Perth en Dundee, Schotland:

- Doppler ultrageluidsonderzoek van de utero-placentaire circulatie

- Evaluatie van echoscopische screening op neurale buis defecten

1989

artsexamen, Rijksuniversiteit Limburg, Maastricht

1989-1993 assistent in opleiding (AIO), vakgroep Gynaecologie en Obstetrie, Academisch Ziekenhuis Maastricht

1994-heden assistent geneeskundige in opleiding (AGIO), vakgroep Gynaecologie en Obstetrie, Academisch Ziekenhuis Maastricht 


\section{Publications}

S.J. Nienhuis, J.M.G. van Vugt, H.J. Hoogland, C.J. Ruissen, J. de Haan. Interexaminer variability of fetal Doppler velocity waveforms. Gynecologic Obstetric Investigation 1988; 25: 152-7. Tevens opgenomen in: JMG van Vugt. Fetal artery Doppler velocimetry. Proefschrift, Maastricht 1988.

J.M.G. van Vugt, C.J. Ruissen, S.J. Nienhuis, H.J. Hoogland, J. de Haan. Comparison of blood velocity waveform indices recorded by Pulsed Doppler en CW Doppler in the umbilical artery. Journal of Clinical Ultrasound 1988; 16: 573-6.

Rapport voor de Gezondheidsraad: H.J. Hoogland, S.J. Nienhuis, C.J. Ruissen. Neurale buisdefecten. Voorstudie, 1990.

Abstract: C.J. Ruissen, S.J. Nienhuis, H.J. Hoogland, J.S.H. Vles, W.J. Gerver, J. de Haan. Cost-effectiveness of a Doppler based policy of suspected intrauterine growth retardation. A randomized controlled trial. Journal of Maternal Investigations 1991; 1,2: 126 .

Abstract. S.J. Nienhuis, C.J. Ruissen, H.J. Hoogland, J.S.H. Vles, W.J. Gerver, J. de Haan. Cost-effectiveness of a Doppler policy of suspected intrauterine growth retardation. A randomized controlled trial. Gynaeco Selectief 1991; 4: 5.

Boekbespreking. S.J. Nienhuis. Jelle Banga (1786-1877), door Prof. dr M.J. van Lieburg. Nederlands Tijdschrift voor Obstetrie en Gynaecologie 1992; 3: 147.

J.J.P.M. Pieters, S.J. Nienhuis, I. Rabelink, M. Kessels, C.J. Ruissen, L. Volovics, H.J. Hoogland. Foetale biometrie en placentameting in het tweede trimester van de graviditeit als parameter voor de vroege predictie van intra- en extra-uteriene groeiproblemen. Nederlands Tijdschrift voor Obstetrie en Gynaecologie 1993; 106: 67-8.

I.A.A. Rabelink, J.E.M. Degen, M.E.W. Kessels, S.J. Nienhuis, C.J. Ruissen, H.J. Hoogland. Variation of early fetal growth. European Journal of Obstetrics \& Gynecology and Reproductive Biology 1994; 53: 39-43.

S.J. Nienhuis, N.M.A.J. Zusterzeel, H.J. Hoogland. Routine clinical policy and application of Doppler measurements in suspected intrauterine growth retardation in university hospitals in the Netherlands. European Journal of Obstetrics \& Gynecology and Reproductive Biology 1994; 56: 31-6. 
. 
